\title{
ADULT ATTACHMENT AND SPOUSAL REACTIONS TO MILITARY DEPLOYMENT SEPARATIONS AND REUNIONS
}

By

\author{
Valerie M. Wood
}

A dissertation submitted to the Department of Psychology

In conformity with the requirements for

the degree of Doctor of Philosophy

Queen's University

Kingston, Ontario, Canada

(April, 2017)

Copyright () Valerie M. Wood, 2017 


\begin{abstract}
The purpose of my dissertation was to assess the relevance of adult attachment in explaining spousal adjustment and relationship functioning in military deployment experiences. Specifically, I was interested in identifying what attachment dimensions are related to spousal coping and relationship perceptions during deployment separations and reunions, when and how attachment dimensions are related to outcomes during across the deployment cycle, and why attachment dimensions are related to such outcomes. This project was sponsored by the Director General Military Personnel Research and Analysis (Department of National Defence) and consisted of three phases. Phase One was crosssectional, examining civilian spouses/partners of military members experiencing a deployment separation (Group A), and civilian spouses/partners of military members experiencing a deployment reunion (Group B). Group A individuals were invited to participate in a longitudinal study, following them monthly across the separation, as Phase Two. Phase Three consisted of a large-scale survey sent to spouses/partners of military members capturing several indices of coping and relationship functioning for spouses of varying partner deployment status'. In Phase One, for Group A, attachment anxiety was related to compromised coping and relationship perceptions during the separation, and attachment avoidance related to increased coping, but negative relationship perceptions. The relationships between attachment anxiety and relationship perceptions were moderated by time deployed and experience with deployments. For Group B, attachment anxiety was related to decreased coping and negative relationship perceptions during the reunion. The relationships between attachment anxiety and relationship perceptions were mediated by expectations of the return, and were moderated
\end{abstract}


by time reunited. In Phase Two, attachment avoidance was related to negative relationship perceptions including difficulties with emotional support. In Phase Three, attachment anxiety, attachment avoidance, and their interaction was related to indices of coping and relationship functioning. Further, some of these relationships were moderated by environmental conditions. Notably, recent deployment status moderated relationships among attachment dimensions and perceived relationship and coping outcomes. Finally, I found that emotional fitness mediated relationships among attachment anxiety and coping outcomes, and perceptions of partner support mediated relationships among attachment anxiety and relationship outcomes. Practical and theoretical implications and future directions are discussed. 


\section{Acknowledgements}

The road to my $\mathrm{PhD}$ completion has certainly been as smooth, sane, enjoyable, and successful, as possible because of the incredible supervision of you, Dr. Tara MacDonald. Thank you, a million times over, for being the best role model, friend, and colleague a graduate student could ask for. Conducting research with you has been fun, inspiring, and motivating, I haven't stopped learning from you even at this point. Your continued emotional support is beyond your role, but has been every bit a part of my success, as has been your academic mentorship. I will look back on these years as some of my best, because of you.

Dr. Lee Fabrigar, my second supervisor, your wisdom and continued passion for the field is unparalleled and has been a major source of inspiration for me. I feel so fortunate for the important lessons you have taught me, the time you have taken to do so, and genuine desire to see me succeed. Your challenging approach, however uncomfortable at times, has made me a more humble and wise researcher, which I will forever value, thank you!

Dr. Ronald Holden, thank you for being on my committee, and for your continued support and encouragement during my graduate years. I have appreciated your humorous nature, wisdom, and great advice, thank you for making the dissertation defense process a positive experience for me.

I would be remiss without acknowledging someone who, behind the scenes, has been a huge source of support, encouragement, and knowledge throughout my graduate studies, Dr. Danielle Charbonneau. You have been such an important part of my graduate studies, and life in general. Thank you for all of your guidance and support, not to 
mention my professional development at the Royal Military College, and for the discussion that lead to this research idea entirely!

Mom, Dad, and Heather, you are the reason that I value education and professional development to the extent that I do, I cannot thank you enough for instilling these values in me, and encouraging my continued educational success. You have all helped to keep me motivated and working towards my goals, thank you for all of your love and support.

Finally, to Danny, my husband and very best supporter. It is hard to believe that we met during my graduate studies, are now married, and about to start a family together. Thank you for being such a stable, positive, and encouraging part of my life, which has no doubt given me the efficacy and secure base that I have needed to accomplish all of my goals. I find it very symbolic to have achieved a sense of attachment security that I research, write, and teach about, through you. 


\section{Disclaimer}

The opinions expressed in this publication reflect the opinion of the author and do not represent the opinion of the Canadian Armed Forces $(C A F)$ or the Department of National Defence. In addition, these findings may not be generalizable to all CAF spouses/partners. 


\section{Table of Contents}

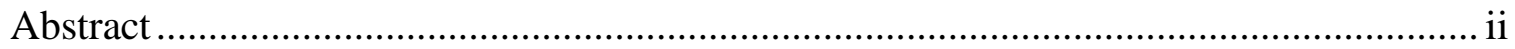

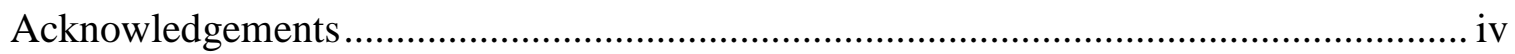

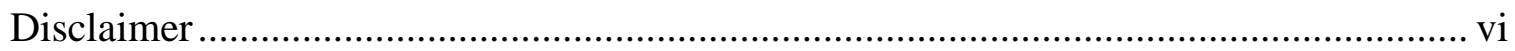

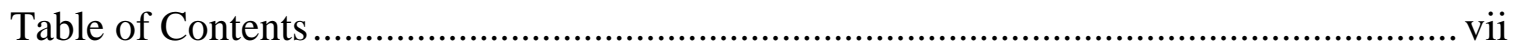

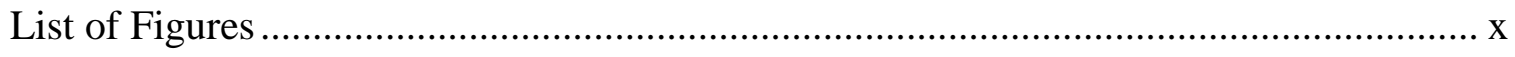

List of Tables ............................................................................................... xiii

Chapter 1: General Introduction and Overview ....................................................... 14

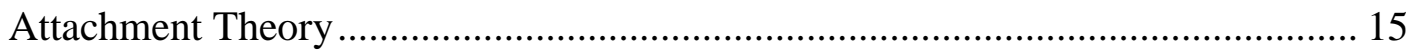

Attachment and Reactions to Separations and Reunions .................................... 18

The Relevance of Attachment Theory to Military Deployments .......................... 21

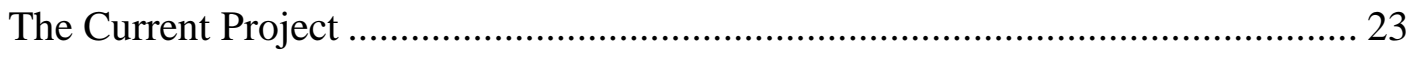

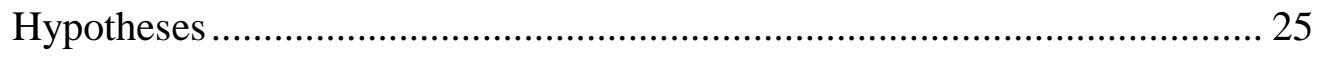

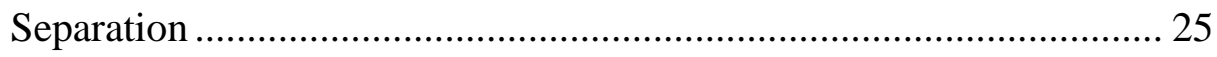

Stages of Spousal Adjustment to Military Deployments ........................... 27

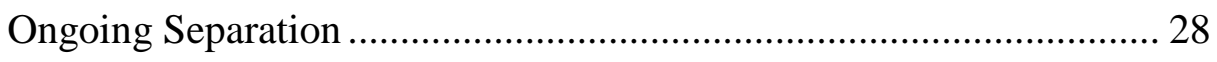

Reunion.................................................................................. 31

Large-Scale Survey................................................................. 34

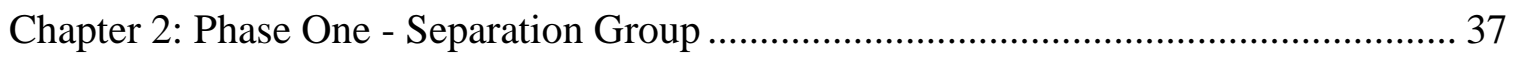

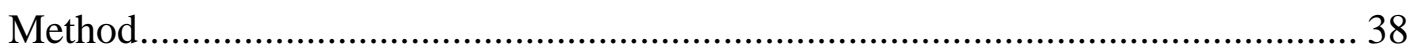

Participants and Procedure ................................................................. 38

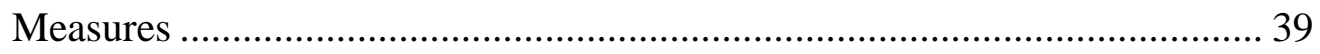

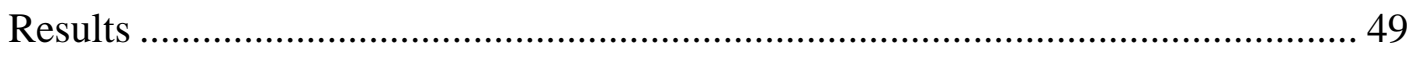

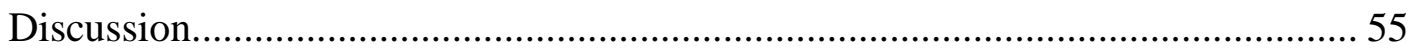

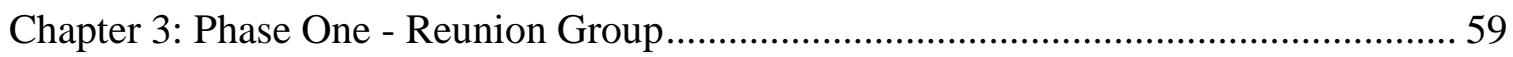

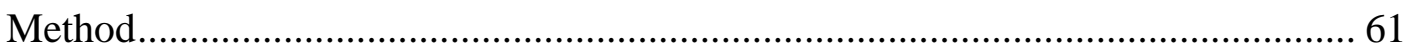

Participants and Procedure ................................................................. 61

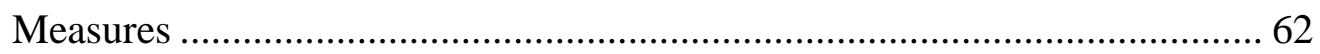

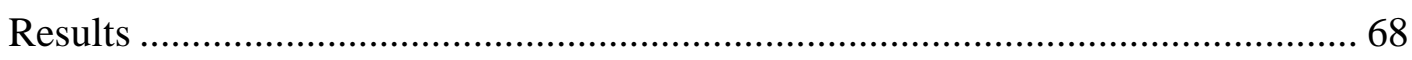

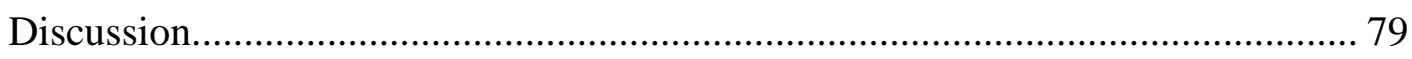

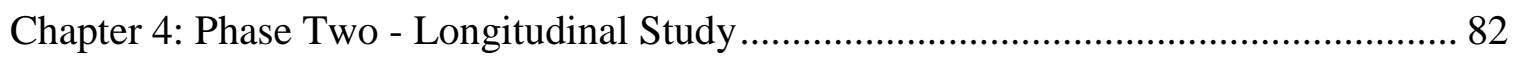

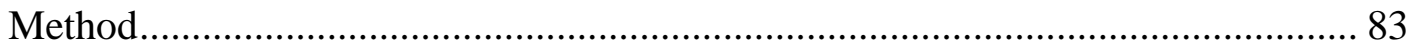

Participants and Procedure ............................................................... 83

Monthly Survey Measures .................................................................... 84 
Reunion Survey Measures ...................................................................... 92

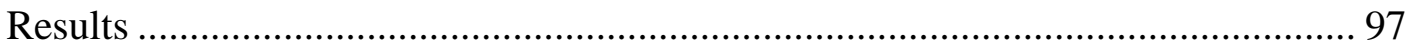

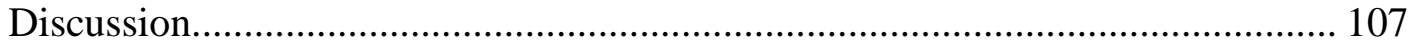

Chapter 5: Phase Three - Large-Scale Survey ............................................................... 111

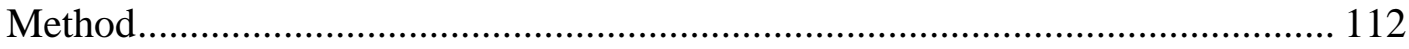

Participants and Procedure ........................................................................ 112

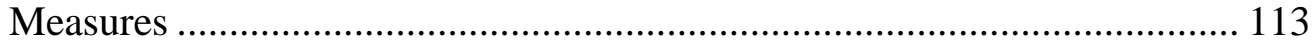

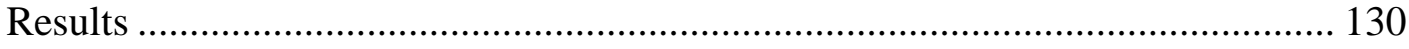

Attachment Anxiety and Avoidance as Predictors ...................................... 131

Attachment Anxiety, Avoidance, and Deployment Status ........................... 140

Attachment Anxiety, Avoidance, and Recent Deployment Status .............. 145

Attachment Anxiety, Avoidance, and Months Deployed ............................ 155

Curvilinear Effects of Months Deployed as a Function of Attachment Anxiety and Attachment Avoidance ......................................................... 161

Curvilinear Effects of Attachment Anxiety and Attachment Avoidance as a Function of Months Deployed....................................................... 164

Attachment Anxiety, Avoidance, and Deployment Prep Status .................. 165

Attachment Anxiety, Avoidance, and Recent Reunion Status...................... 168

Attachment Anxiety, Avoidance, and Months Reunited ............................. 173

Curvilinear Effects of Months Reunited as a Function of Attachment Anxiety and Attachment Avoidance ........................................................... 177

Mediation Models for Attachment Anxiety and Related Outcomes............ 181

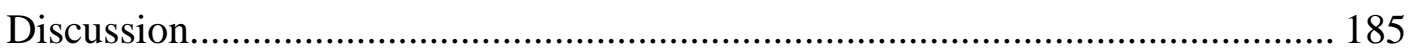

Deployment Separation Findings .............................................................. 188

Deployment Reunion Findings …………................................................... 195

Mechanisms of Attachment Anxiety on Intrapersonal and Relationship

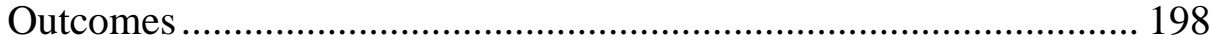

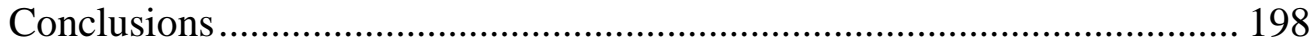

Chapter 6: General Discussion................................................................................. 202

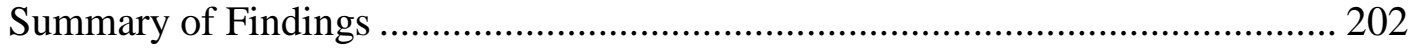

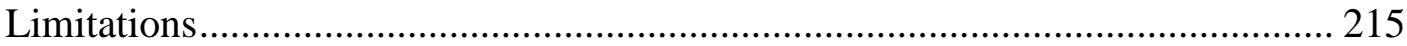

Contribution to Previous Literature..................................................................... 219

Implications and Future Directions ............................................................. 225

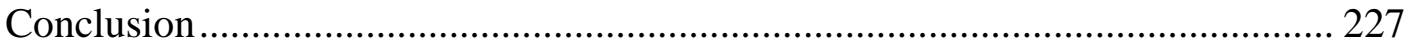

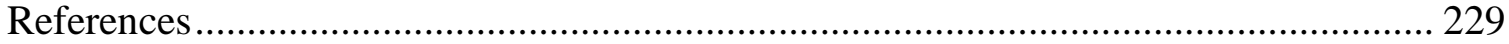


Appendix A: The Experiences in Close Relationships Scale - Revised .......................... 242

Appendix B: The Rosenberg Self Esteem Scale .......................................................... 243

Appendix C: The Relationship Assessment Scale ........................................................ 244

Appendix D: The State Trait Anxiety Inventory …………………………………..... 245

Appendix E: The Positive and Negative Affect Schedule ............................................ 246

Appendix F: Relationship Quality Measure ………………................................... 247

Appendix G: Coping Capacity Measure for Phase One ……………………….......... 248

Appendix H: Reunion Experiences Measure ............................................................ 249

Appendix I: Coping Capacity Measure for Phase Two …………………………...... 251

Appendix J: Open-Ended Responses for Phase One Reunion Survey .......................... 252

Appendix K: Impact of Military Life on Career Measure for Phase Three ..................... 253

Appendix L: Stress During Military Experiences Measure for Phase Three.................. 254

Appendix M: Stress Measure for Phase Three ……………...................................... 255

Appendix N: The Satisfaction With Life Scale …………………………………......... 256

Appendix O: The Kessler Psychological Distress Scale ............................................... 257

Appendix P: The Pearlin Mastery Scale …………………..................................... 258

Appendix Q: The Brief COPE Scale ………………............................................. 259

Appendix R: Support and Resiliency Inventory ………............................................. 261

Appendix S: Quality of Marriage Index ................................................................... 263

Appendix T: The Revised Dyadic Adjustment Scale ……………………………...... 264

Appendix U: Queen's General Research Ethics Board Letter of Approval for Phases

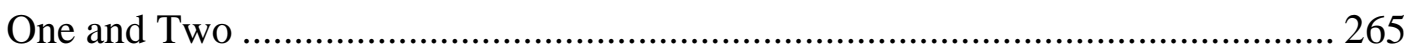

Appendix V: Queen's General Research Ethics Board Letter of Approval for Phase Three 


\section{List of Figures}

Figure 1: Deployment Cycle for Families (Egerton-Graham, 2009)

28

Figure 2: Scree plot of the reduced correlation matrix for relationship quality items (Phase One separation)

Figure 3: Scree plot of the reduced correlation matrix for coping capacity items (Phase One separation)

Figure 4: Interaction between attachment anxiety and previous deployment status on perceived relationship harmony during the deployment separation

Figure 5: Interaction between attachment anxiety and number of days separated on relationship satisfaction during the deployment separation.

Figure 6: Interaction between attachment anxiety and number of days separated on perceived relationship harmony during the deployment separation

Figure 7: Scree plot of the reduced correlation matrix for coping capacity items (Phase One reunion)

Figure 8: Interaction between attachment anxiety and number of days reunited on relationship satisfaction

Figure 9: Interaction between attachment anxiety and number of days reunited on quality of partner interactions

Figure 10: Unstandardized regression coefficients for the relationship between attachment anxiety and relationship satisfaction as mediated by expectations of the reunion being met

Figure 11: Unstandardized regression coefficients for the relationship between attachment anxiety and quality of partner interactions as mediated by expectations of the reunion being met

Figure 12: Unstandardized regression coefficients for the relationship between attachment anxiety and satisfaction with one's partner relative to the time before the deployment as mediated by expectations of the reunion being met

Figure 13: Scree plot of the reduced correlation matrix for coping capacity items (Phase Two first month)

Figure 14: Scree plot of the reduced correlation matrix for emotional support items (Phase Two first month)

Figure 15: Scree plot of the reduced correlation matrix for expectations for the reunion items (Phase Two first month)

Figure 16: Scree plot of the reduced correlation matrix for coping capacity items (Phase Two first month)

Figure 17: Interaction between attachment anxiety and attachment avoidance on relationship satisfaction

Figure 18: Interaction between attachment anxiety and attachment avoidance on the extent to which expectations of the reunion were met 
Figure 19: Interaction between attachment anxiety and attachment avoidance on feeling more versus less satisfied with one's relationship relative to the time before the deployment.. 101

Figure 20: Interaction between attachment anxiety and attachment avoidance on feeling more versus less emotionally connected to one's partner relative to the time before the deployment ....

Figure 21: Interaction between attachment anxiety and attachment avoidance on feeling as though things are running more versus less smoothly around the house relative to the time before the deployment

Figure 22: Scree plot of the reduced correlation matrix for impact of military life on career items (Phase Three)

Figure 23: Scree plot of the reduced correlation matrix for stress during military experiences items (Phase Three)

Figure 24: Scree plot of the reduced correlation matrix for stress items (Phase Three)

Figure 25: Scree plot of the reduced correlation matrix for coping strategies items (Phase Three)

Figure 26: Interaction between attachment anxiety and attachment avoidance on the use of social support as a coping strategy

Figure 27: Interaction between attachment anxiety and attachment avoidance on the use of selfblame as a coping strategy....

Figure 28: Interaction between attachment anxiety and attachment avoidance on the use of selfdistraction as a coping strategy.

Figure 29: Interaction between attachment anxiety and attachment avoidance on perceived relationship support

Figure 30: Interaction between attachment anxiety and attachment avoidance on relationship success

Figure 31: Interaction between attachment anxiety and attachment avoidance on relationship satisfaction (QMI)

Figure 32: Interaction between attachment avoidance and deployment status on perceived relationship support

Figure 33: Interaction between attachment avoidance and deployment status on cognitive fitness

Figure 34: Interaction between attachment avoidance and recent deployment status ( $<1$ mon) on perceived relationship support.

Figure 35: Interaction between attachment avoidance and recent deployment status $(<1$ mon $)$ on cognitive fitness

Figure 36: Interaction between attachment avoidance and recent deployment status $(<1$ mon $)$ on perceived relationship success.

Figure 37: Interaction between attachment avoidance and recent deployment status $(<1$ mon $)$ on relationship satisfaction (RDAS) 
Figure 38: Interaction between attachment avoidance and recent deployment status ( $<1$ mon) on

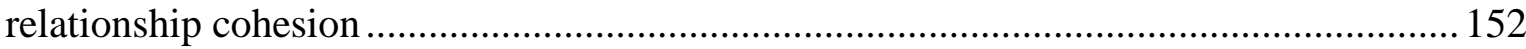

Figure 39: Interaction between attachment anxiety and recent deployment status $(<1 \mathrm{mon})$ on perceived relationship support.

Figure 40: Interaction between attachment anxiety and recent deployment status ( $<1$ mon) on cognitive fitness.

Figure 41: Interaction between attachment anxiety and recent deployment status $(<1$ mon) on perceived relationship success

Figure 42: Interaction between attachment anxiety and recent deployment status $(<1 \mathrm{mon})$ on relationship satisfaction (RDAS)

Figure 43: Interaction between attachment avoidance and number of months deployed (transformed and mean centered) on reported stress during general military experiences 159

Figure 44: Interaction between attachment anxiety and number of months deployed (transformed and mean centered) on relationship success.... 159

Figure 45: Interaction among attachment anxiety, attachment avoidance, and number of months deployed (transformed and mean centered) on stress during general military experiences

Figure 46: Interaction between attachment anxiety, attachment avoidance, and number of months deployed (transformed and mean centered) on coping success.

Figure 47: Interaction between attachment avoidance and number of months deployed (transformed and mean-centered) squared on the use of self-blame as a coping strategy

Figure 48: Interaction between attachment anxiety and number of months deployed (transformed and mean-centered) squared on relationship satisfaction (QMI) 164

Figure 49: Interaction between attachment anxiety and recent reunion status on perceived relationship success 172

Figure 50: Interaction between attachment anxiety and recent reunion status on relationship consensus

Figure 51: Interaction between attachment avoidance and months reunited on life mastery ..... 176

Figure 52: Interaction between attachment anxiety and months reunited on life mastery ......... 176

Figure 53: Interaction between attachment avoidance and months reunited squared on relationship success 178

Figure 54: Interaction between attachment avoidance and months reunited squared on relationship satisfaction using the QMI measure

Figure 55: Interaction between attachment anxiety and months reunited squared on life readiness 180

Figure 56: Interaction between attachment anxiety and months reunited squared on relationship success 


\section{List of Tables}

Table 1: Descriptive Statistics for Separation Measures ................................................................ 49

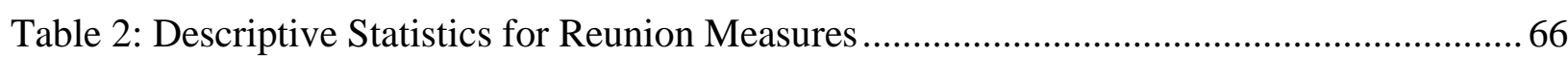

Table 3: Bootstrap Results for the Indirect Effects of Attachment Anxiety on Listed DVs

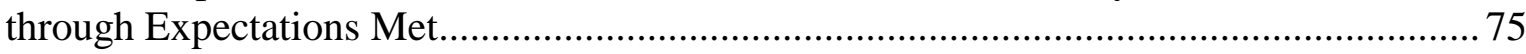

Table 4: Descriptive Statistics for Monthly Survey Measures ………………………….............. 92

Table 5: Descriptive Statistics for Reunion Measures for Phase Two Group ...............................96

Table 6: Descriptive Statistics for Quality of Life Measures ..................................................... 129

Table 7: Standardized Regression Coefficients for the Unique and Interactive Effects of Attachment Anxiety and Avoidance on all Dependent Measures .................................... 134

Table 8: Mediation Models for Relationships Between Attachment Anxiety and Coping Outcomes as Mediated by Emotional Fitness ………………......................................... 184

Table 9: Mediation Models for Relationships Between Attachment Anxiety and Relationship Perceptions as Mediated by Perceived Relationship Support ............................................ 185 


\section{Chapter 1}

\section{General Introduction and Overview}

Why some individuals should recover, largely or completely, from experiences of separation and loss while others seem not to is a central question, but one not easily answered. (Bowlby, 1973, p. 23).

Although it is recognized that being deployed can affect the physical and psychological well-being of military personnel, it is less appreciated that their absences also profoundly affect family members who remain at home. Families of deployed personnel are often called the “overlooked casualties of conflict” (The Royal Navy and Royal Marines Children's Fund, 2009) because they face a number of unique challenges over the course of a deployment. Indeed, various challenges arise at different stages of the deployment process as couples prepare for deployment, as they are separated for the duration of deployment, and when they are reunited. Such difficulties include adjusting to the member's deployment in a variety of domains (e.g., parenting styles, household and employment decisions, changes to the nature of the relationship) and then readjusting upon the member's return.

Previous work on the impact of deployments on spouses has often evaluated individuals at one point in time during the deployment cycle (e.g., prior to deployment, during deployment, or following reunion). Many researchers in this area, both inside and outside the Canadian Armed Forces (CAF), have recognized this limitation (e.g., De Burgh et al., 2011; Chandra et al., 2008). Therefore, they have stressed the need for research to follow military couples throughout the deployment cycle to see whether specific stages of it are more difficult to experience than others.

In addition to assessing whether particular deployment stages are especially challenging for military couples, it is also valuable to investigate whether individual differences are 
associated with adjustment to deployment. In other words, are specific traits associated with the ability to cope emotionally, physically, and practically with life's challenges before, during, and after a partner has deployed? For my dissertation, I explore whether spouses' attachment orientations predict how they adjust to challenges faced at all stages of the deployment process (at the initial separation, during the deployment, and at the reunion). Attachment theory has contributed a great deal to the understanding of relationship processes and serves as the theoretical framework for my research.

\section{Attachment Theory}

Attachment theory was originally proposed by Bowlby $(1969,1973)$ to explain the close bonds that develop between infants and their primary caregivers. Bowlby believed that through early interactions with their primary caregivers (called attachment figures) infants begin to generate expectations and beliefs regarding their caregivers' availability and responsiveness.

Here, an infant begins to learn whether he or she can depend on their caregiver for physical and emotional security. Further, Bowlby explained that this process is universal such that all individuals possess an attachment behavioural system which functions to regulate individuals' emotions and behaviour under conditions of threat. The goal of all infants is to achieve emotional security. Specifically, during infancy individuals do not yet possess the cognitive capacity required to cope with environmental threats and will instinctively seek proximity to their caregivers under stressful or dangerous conditions. Although some infants receive adequate emotional support, others do not and develop insecurities regarding the availability of attachment figures and display ineffective emotion regulation strategies in response to stressors. A secure attachment occurs when the infant can successfully achieve emotional security by seeking proximity to the attachment figure, and the attachment figure is consistently available 
and responsive. An insecure attachment occurs when the infant seeks proximity to the attachment figure, yet is not consistently met with an available or responsive attachment figure (Cassidy, 2008). For these insecure infants, they develop other strategies to achieve security, either by engaging in 'hyperactivating' strategies aimed at escalating attachment to ensure their caregivers take notice (e.g., escalating distress-signalling behaviours), or by engaging in 'deactivating' strategies aimed at inhibiting signs of distress and experiencing potential rejection from their caregiver (e.g., avoiding caregivers, learning to manage the threat independently, even if ineffectively).

Ainsworth (Ainsworth, Blehar, Waters, \& Wall, 1978) was the first to classify differences in infant-parent attachment behaviour using the Strange Situation Procedure which classifies infants according to their behavioural responses to a number of scenarios, involving separations and reunions with the caregiver as well as encounters with a stranger. Secure infants become noticeably upset when separated from their caregivers, however seek proximity to their caregivers, and greet them warmly, upon reunion. These infants tend to have caregivers who are more sensitive and responsive relative to caregivers of insecurely attached infants (De Wolff \& Van Ijzendoorn, 1997). Resistant infants demonstrate strong separation protest following separation from a caregiver (albeit a sign of their use of hyperactivating strategies) and also show ambivalence in desiring proximity to, yet resisting contact with, their caregivers upon reunion. These infants often have mothers who are inconsistent in their caregiving and who are often rejecting (De Wolff \& Van Ijzendoorn, 1997; Isabella, 1993). Avoidant infants show little separation protest (a sign of their use of deactivating strategies), and often ignore their caregiver upon their return. Mothers of avoidant infants tend to be rejecting, insensitively under or over stimulating, and tend to think quite negatively of their infants (Belsky, Rovine, \& Taylor, 1984). 
Finally, an infant is classified as disorganized in the Strange Situation when he/she shows disorganized or disoriented behaviours because these infants have likely not developed a coherent emotional regulation strategy in response to attachment-related threats. One predictor of infant disorganized attachment is parental frightening behaviour (see Van Ijzendoorn, Schuengel, \& Bakermans-Kranenburg, 1999 for a review). Because this type of attachment is associated with an absence of organized emotion regulation strategies, these infants (and later, adults) experience the most poorly managed emotions (see Lyons-Ruth \& Jacobvitz, 2008). More recently, attachment theory has been applied to the understanding of adult romantic love (Hazan \& Shaver, 1987, 1994). Adult attachment theory is guided by the assumption that the very motivational system which regulates emotional bonds between infants and their primary caregivers also regulates the bonds that develop between adult romantic partners. This logic is based on Bowlby's assertion that we develop 'internal working models' (internalized expectations and beliefs regarding the responsiveness and availability of others) that later shape interactions with significant others. Indeed, much research supports the view that attachment styles tend to be stable across time and relationships (Baldwin \& Fehr, 1995; Fraley, 2002; Scharfe \& Bartholomew, 1994; Waters et al., 2000). In other words, individuals' expectations about how responsive others will be to their needs, how dependable others are, and how relationships typically function, tend to be internalized and are fairly stable over time.

The way that attachment orientations are assessed in adults has evolved considerably since the Hazan and Shaver's seminal (1987) contribution. Currently, there is consensus that, adult attachment is best assessed along two independent dimensions: attachment avoidance and anxiety (Fraley, Waller, \& Brennan, 2000). Attachment avoidance reflects the extent to which individuals are uncomfortable with emotional closeness and intimacy and prefer emotional 
independence in a relationship. Attachment anxiety represents the degree to which individuals are preoccupied by thoughts of rejection and abandonment by their partners and, as a result, how much they feel they must monitor their environment for signs of disapproval or loss of interest (Mikulincer, Gillath, \& Shaver, 2002). Attachment anxiety also captures the extent to which individuals feel unworthy of love and, as a result, seek constant reassurance and validation from their partners. For my dissertation, I focus on the role of attachment anxiety and attachment avoidance in shaping spouses' experience of a deployment.

\section{Attachment and Reactions to Separations and Reunions}

According to Bowlby (1982), upon encountering physical or psychological threats the attachment system becomes activated such that infants become motivated to maintain or restore proximity to their attachment figures. Mikulincer, Gillath, and Shaver (2002) demonstrated that similar processes occur in adulthood. In particular, the adult attachment system becomes activated under conditions of attachment-related threat (e.g., potential or experienced loss of an attachment figure) as well as general threat (e.g., stress or physical danger). Such conditions automatically and implicitly activate thoughts of attachment figures that can motivate an individual to increase physical or psychological proximity to them. In other words, working models of attachment should regulate attachment behaviour (e.g., proximity seeking strategies, distress-signalling, or distancing). Indeed, individual differences in attachment behaviour should be most pronounced under stressful conditions in the social or physical environment (Simpson \& Rholes, 1994).

By definition, attachment anxiety is associated with fears of rejection and abandonment from attachment figures (Fraley \& Waller, 1998). Due to their persistent insecurities and doubts about others' responsiveness, it is understood that highly anxious individuals experience chronic 
hyperactivation of the attachment system (Mikulincer, Gillath, \& Shaver, 2002; Mikulincer \& Shaver, 2007). In this sense, anxious individuals are preoccupied with thoughts of potential rejection and therefore vigilantly monitor their environment for cues of disapproval or loss of interest, and experience heightened cognitive accessibility of attachment figures (Mikulincer, Gillath, \& Shaver, 2002). Indeed, research has demonstrated that highly anxious individuals are hypervigilant in the detection and processing of rejection-related threat cues (Mikulincer \& Shaver, 2003, 2007), can quickly access words related to proximity and distance (e.g., acceptance and rejection; Mikulincer, Birnbaum, Woddis, \& Nachmias, 2000; Mikulincer, Gillath, \& Shaver, 2002), experience difficulty in inhibiting expectations for rejection (Baldwin \& Kay, 2003), as well as suppressing thoughts related to partner separation (Mikulincer, Dolev, \& Shaver, 2004) relative to individuals low in anxiety.

Given that attachment anxiety is associated with enhanced detection and processing of rejection related stimuli, one might expect that highly anxious individuals would also be more reactive to actual experiences of rejection and separation from one's romantic partner.

Consistent with this idea, individuals high in attachment anxiety tend to become more distraught following a romantic breakup than those lower in attachment anxiety (Davis, Shaver, \& Vernon, 2003; Feeney \& Noller, 1992). Attachment anxiety is also predictive of reactions to physical separations from romantic partners. For example, Fraley and Shaver (1998) examined couples as they parted in an airport (i.e., one partner was boarding a plane and the other one was staying behind) and found that women high in attachment anxiety reported more distress as they bade their partner good-bye relative to women low in attachment anxiety. In addition, an anxious attachment style is associated with emotion-focused coping strategies in response to divorce and increased signals of distress towards romantic partners (e.g., Birnbaum, Orr, Mikulincer, \& 
Florian, 1997; Meyer, Olivier, and Roth, 2005). Mayseless, Danieli, and Sharabany (1996) found that an anxious attachment style was related to intense distress and self-blaming in response to imagined separations. Thus, it seems as though highly anxious individuals are acting in accordance with their internal working models. Specifically, just as anxious-resistant infants show strong separation protest to separation with their mothers, anxious adults show similar hyperactivating responses including greater distress when separated from their romantic partners, relative to less anxious adults.

Attachment avoidance, on the other hand, should be associated with deactivating strategies aimed at promoting interpersonal distance when the attachment system is activated (e.g., stress or attachment-related threats). However, under conditions of low threat attachment avoidance should not be as strongly related to distancing behaviours. Consistent with this idea, Simpson, Rholes, and Nelligan (1992) found that avoidant women pulled away from their partners only when they were anticipating a stressful task, but not when they were anticipating a neutral task. In Fraley and Shaver's airport study, avoidant women were also behaving in accordance with their working models. The highly avoidant women who were anticipating a separation did not report greater distress relative to less avoidant women, but they showed distancing behaviours by pulling away from their partners more and avoiding close contact relative to less avoidant women. Finally, in response to imagining separations with a partner Mayseless and colleagues (1996) found that avoidant attachment was associated with refraining to manage the threat. This suggests that highly avoidant individuals engage in distancing behaviours when the attachment system is activated (i.e., in times of stress) in order to reduce their physiological arousal and experienced distress. 
Some researchers have questioned the success of deactivating strategies at regulating emotional responses to threats, attachment-related threats in particular. Research with infants (Sroufe \& Waters, 1977) seems to suggest that defensive strategies are not associated with decreases in physiological arousal, and that the pattern of heart rate acceleration for infants that displayed defensive strategies (physical distancing from their attachment figure) following a separation were similar to the infants who were highly distressed. However, research with avoidant adults suggests that efforts to suppress thoughts of attachment-related threats like separation or potential loss of a romantic partner are successful, and such strategies lead to decreased activation of thoughts related to abandonment (Fraley \& Shaver, 1997, Study 1), and decreased skin conductance responses (Fraley \& Shaver, 1997, Study 2) relative to thought suppression about neutral stimuli. Therefore, deactivating strategies in response to threats seems to result in successful de-activation of the attachment-related system for avoidant adults.

However, there is evidence to suggest that when facing a more serious stressor such as a life transition, avoidant individuals may show ineffective coping strategies and psychological distress, and may resemble anxious-ambivalent individuals in terms of affect and behaviour (Mikulincer \& Florian, 1998). Specifically, in a study examining the impact of mothers' attachment styles on coping and adjustment to the birth of their first child, Mikulincer and Florian (1998) found that, similar to anxious-ambivalent mothers, avoidant first-time mothers reported higher levels of distress, and described the task of being a mother in more threatening terms than control mothers.

\section{The Relevancy of Attachment Theory to Military Deployments}

The stages of a deployment can be viewed as analogous to those included in Ainsworth's Strange Situation Procedure (initial separation, introduction of a threat, and reunion with the 
caregiver). Therefore, military deployments are a relevant and unique context within which to study the principles of attachment theory in adult romantic relationships. Although some research has shown that attachment styles predicts one's cognitive and emotional reactions to military and war-related stressors (e.g., Mikulincer, Florian, \& Wellar, 1993; Mikulincer \& Florian, 1995), there has been little work done examining the role of attachment styles in predicting reactions to a romantic partner's military deployment. Although previous work has shown that attachment anxiety predicts reactions to separations with romantic partners (Fraley and Shaver, 1998), the separations examined in previous research were unlike those experienced by military personnel and their partners. Indeed, the experiences of military families are unlike those experienced by most civilian families (see Ainspan, Bryan, \& Penk, 2016). Deployments can occur for a number of reasons (e.g., training, peacekeeping, disaster relief, humanitarian operations), can entail various and unpredictable levels of threat to one's safety, and can occur with little advance notice. Therefore, military couples are not always able to predict their separations, how long their separations will last, what the separation will entail, and even the possibility of psychological consequences such as posttraumatic stress disorder. Further, deployments could entail periods of high operational tempo (high demand, long work hours, unpredictable schedules) making communication efforts difficult. In other words, a partner's availability and responsiveness might be unpredictable, the crucial pillars of a secure attachment. If military partners are experiencing endangerment or exposing a service member to traumatic experiences, they may not be able to fulfill their spouses' attachment needs.

Cafferty and colleagues (1994) examined the relevance of attachment orientations in the functioning of couples four months after being reunited following a military deployment. Specifically, the researchers looked at relationships between attachment orientations (assessed 
using Hazan and Shaver's typological approach), relationship satisfaction, and levels of positive and negative affect in military male partners and their female spouses who remained at home. Secure individuals in general (women and men) reported less conflict and more satisfaction than preoccupied partners' post-reunion, but secure men only (not their female spouses) reported greater positive, and less negative, affect during the post-reunion period. Although these initial findings suggest that attachment orientations are relevant in the context of military separations, many answers remain. For example, these researchers used the typological approach that does not allow for a more accurate assessment of the unique and interactive effects of attachment anxiety and avoidance that is possible with the more recent dimensional approach. Second, these researchers only captured the post-reunion period so did not explore the relevance of attachment processes during the separation itself. Third, these researchers did not explore or identify potential mechanisms that might explain the relationships between attachment security and postreunion difficulties. Finally, this study did not include a comparison group or a longitudinal component so it is difficult to determine whether attachment dimensions are relevant in explaining relationship outcomes during transitional periods or whether attachment dimensions are chronically relevant across all deployment stages and relationship processes.

\section{The Current Project}

I initiated the current project in collaboration with the Royal Military College of Canada, Director General Military Personnel Research and Analysis (DGMPRA), and Director Military Family Services (Department of National Defence). The purpose of this project was to examine how attachment anxiety and attachment avoidance predicted the functioning of at-home civilian spouses who are being separated from partners due to a military deployment. More specifically, I was interested in assessing how attachment anxiety and attachment avoidance predict the 
affective, cognitive, and behavioural reactions of spouses across various stages of the deployment cycle (i.e., initial separation, ongoing separation, and the reunion).

There are three phases to this project. The first phase consisted of a cross-sectional design whereby DGMPRA recruited civilian spouses/partners of those who were Regular Force (not Augmentees or Reservists ${ }^{1}$, or dual-service couples) who were separated from their military spouses/partners due to deployment (Group A), and another similar group of civilian/civilian spouses/partners who had recently experienced a reunion with their military spouses/partners due to a deployment (Group B). The purpose of this first phase was to examine the effects of adult attachment on reactions to both a deployment separation and reunion. At the end of Group A's separation questionnaire, these individuals were invited to participate in a longitudinal study which comprised the second phase of this project. The purpose of this phase was to examine the effects of attachment on spousal functioning during the ongoing separation. Group A participants were also asked to complete a final questionnaire shortly after they were reunited with their partners to assess functioning during the reunion phase similar to Group B. In the third and final phase of the project I examined how attachment dimensions moderated the impact of military life on spouses of military members in a large-scale survey. This survey captured a number of indices of spouse's perceptions of their intrapersonal and interpersonal (relationship) functioning for spouses of varying partner deployment status' (spouses of partners not currently deployed, deployed, preparing for a deployment, and those recently reunited with their partners from a deployment). The goal of this final project is to elucidate the relationships between attachment styles and spousal functioning on a number of variables indicating their

\footnotetext{
${ }^{1}$ The Reserve Force supports, augments, and sustains the Regular Force in international and domestic operations. Many reservists are employed full-time outside of the CAF in civilian occupations, and others are full-time students (see Canadian Army, 2017).
} 
psychological adjustment, coping strategies, and relationship perceptions, but also to replicate findings from the previous three phases. In particular, I was interested in testing whether the effects of attachment anxiety and avoidance might be exacerbated for those currently experiencing a deployment separation or reunion relative to those not experiencing a separation or reunion, and the potential trajectory of such outcomes as a function of separation or reunion length.

Hypotheses

\section{Separation}

In line with findings from Fraley and Shaver (1998), Mayseless and colleagues (1996), and Cafferty and colleagues (1994), I predicted that individuals higher in attachment anxiety would report greater difficulties during the separation relative to individuals lower in attachment anxiety. To reiterate, individual differences in attachment behaviour should be most pronounced under stressful conditions in the social or physical environment (Simpson \& Rholes, 1994). Further, the separation should be particularly stressful for anxious individuals given their strong desires for closeness and intimacy and their reliance on attachment figures for emotional regulation and support. Indeed, this is a situation that anxious individuals fear the most: abandonment by an attachment figure. High anxiety individuals would likely experience greater adjustments during this phase relative to low anxiety individuals as they learn to cope with being separated from their partner and must be less reliant on their romantic partners for validation and reassurance. High anxiety individuals would likely report higher levels of distress, negative affect, decreased coping capacity and more maladaptive coping strategies (like self-blame: Mayseless et al., 1996) relative to those lower in attachment anxiety. Further, I predicted that 
attachment anxiety will be related to more negative relationship perceptions during the separation in line with previous research (e.g., Collins \& Read, 1990).

According to attachment theory, attachment avoidance should be associated with deactivating strategies in response to environmental threats. Therefore, I predicted that attachment avoidance should not be associated with reported distress or indices of well-being, and, if anything, attachment avoidance might be associated with reports of enhanced emotional well-being during a partner separation given that avoidance is related to self-reliance and autonomy instead of a reliance on attachment figures for stress regulation. On the other hand, it is possible to see some breakdowns in coping capacity and emotional well-being for highly avoidant spouses if military separations are perceived to be high-stress experiences for these individuals in line with findings by Mikulincer and Florian (1998).

It is difficult to make informed predictions regarding potential interactions between anxiety and avoidance for a few reasons. First, researchers who have assessed relationships between attachment styles and cognitive, affective, and behavioural responses to attachmentrelated or attachment-unrelated threats have typically used categorical attachment measures such as Hazen and Shaver's (1987) typological approach (e.g., Burant, Mikulincer, \& Shaver, 2008; Fraley \& Shaver, 1997; Mikulincer \& Florian, 1998; Mikulincer, Florian, \& Wellar, 1993). This poses difficulty in assessing whether the effects of attachment anxiety in particular are exacerbated for those low, relative to high, in attachment avoidance. Some research suggests that both groups experience difficulty with adjustment to trauma or actual partner loss. For example, in one study preoccupied individuals were the least well-adjusted group following the September $11^{\text {th }}$ terrorist attacks (Fraley, Fazzari, Bonanno, \& Dekel, 2006). However, other researchers have found the fearful group to experience the greatest difficulties with psychological 
adjustment and coping after the loss of a spouse (Fraley \& Bonanno, 2004; Wijngaards-de-Meij et al., 2007). Therefore, it is difficult to predict whether the effects of anxiety would be exacerbated for those also high, or low, in avoidance, if interactions between avoidance and anxiety were reported.

\section{Stages of Spousal Adjustment to Military Deployments}

The Canadian Forces Deployment Handbook outlines what reactions are expected throughout the deployment cycle including the initial and ongoing separation and reunion periods (Egerton-Graham, 2009; see Figure 1). The stages and their associated typical responses are based on an empirically-derived model by Logan (1987). Logan argues that one of the most challenging stages is Stage 2 which occurs 1-3 weeks prior to the deployment called detachment and withdrawal and is associated with cognitive, emotional, and relationship difficulties including anxiety, sleep disturbances, regressive behaviours, and ambivalence with displays of intimacy with one's partner. Stage 3, emotional disorganization, occurs during the first 3-6 weeks of the deployment and is also associated with emotional and relationship difficulties including anxiety, emotional instability, clinginess, distrust, and sleep disturbances. However, spouses can expect to experience recovery and stabilization during Stage 4, or 6-12 weeks into the deployment. Given that stages 2 and 3 are perceived to be the most stressful, one might expect individual differences in attachment behaviours to be most pronounced during these stages. 


\begin{tabular}{|c|c|c|c|}
\hline Phase & Stage & Time Frame & $\begin{array}{c}\text { Some Possible Behaviours } \\
\text { Family Members }\end{array}$ \\
\hline \multirow[t]{2}{*}{$\begin{array}{l}\text { Pre- } \\
\text { Deployment }\end{array}$} & $\begin{array}{l}\text { Stage } 1 \\
\text { Anticipation of } \\
\text { loss }\end{array}$ & $\begin{array}{l}6-12 \text { weeks prior } \\
\text { to deployment }\end{array}$ & Easily agitated, angered, frustrated, tired \\
\hline & $\begin{array}{l}\text { Stage } 2 \\
\text { Detachment \& } \\
\text { Withdrawal }\end{array}$ & $\begin{array}{l}1-3 \text { weeks prior } \\
\text { deployment }\end{array}$ & $\begin{array}{l}\text { Lack of focus, difficulty concentrating, } \\
\text { seemingly disinterested, anxious, regressive } \\
\text { behaviours, sleep disturbances }\end{array}$ \\
\hline \multirow[t]{5}{*}{$\begin{array}{l}\text { During } \\
\text { Deployment }\end{array}$} & $\begin{array}{l}\text { Stage 3 } \\
\text { Emotional } \\
\text { Disorganization }\end{array}$ & $\begin{array}{l}\text { First } 3-6 \text { weeks of } \\
\text { deployment }\end{array}$ & $\begin{array}{l}\text { Lack of focus, anxious, overly emotional, } \\
\text { physical symptoms, increased dependence, } \\
\text { insecure, distrustful, sleep disturbances, } \\
\text { resentful, regressive behaviours }\end{array}$ \\
\hline & $\begin{array}{l}\text { Stage } 4 \\
\text { Recovery and } \\
\text { Stabilization }\end{array}$ & $6-12$ weeks & $\begin{array}{l}\text { More relaxed, accepting, happier, open, less } \\
\text { emotional, more attentive, more invested, } \\
\text { overall wellness }\end{array}$ \\
\hline & $\begin{array}{l}\text { Stage } 5 \\
\text { Anticipation of } \\
\text { Homecoming }\end{array}$ & $\begin{array}{l}1-3 \text { weeks prior to } \\
\text { Home Leave Travel } \\
\text { Allowance (HLTA) }\end{array}$ & $\begin{array}{l}\text { Anxious, energetic, impulsive, elated, nervous, } \\
\text { imaginative }\end{array}$ \\
\hline & $\begin{array}{l}\text { Stage } 6 \\
\text { Emotional } \\
\text { Disorganization } \\
\text { (Again!) }\end{array}$ & $\begin{array}{l}\text { First } 3-6 \text { weeks of } \\
\text { re-deployment }\end{array}$ & $\begin{array}{l}\text { Lack of focus, anxious, overly emotional, } \\
\text { physical symptoms, increased dependence, } \\
\text { insecure, distrustful, sleep disturbances, } \\
\text { resentful, regressive behaviours }\end{array}$ \\
\hline & $\begin{array}{l}\text { Stage } 7 \\
\text { Recovery and } \\
\text { Stabilization }\end{array}$ & $6-12$ weeks & $\begin{array}{l}\text { More relaxed, accepting, happier, open, less } \\
\text { emotional, more attentive, more invested, } \\
\text { driven, focused }\end{array}$ \\
\hline \multirow[t]{2}{*}{$\begin{array}{l}\text { Post- } \\
\text { Deployment }\end{array}$} & $\begin{array}{l}\text { Stage } 8 \\
\text { Anticipation of } \\
\text { Homecoming }\end{array}$ & $\begin{array}{l}1-3 \text { weeks prior to } \\
\text { retum of family } \\
\text { member }\end{array}$ & $\begin{array}{l}\text { Anxious, energetic, impulsive, sudden elation, } \\
\text { nervousness, lack of focus, imaginative, happy, } \\
\text { excited }\end{array}$ \\
\hline & $\begin{array}{l}\text { Stage 9 } \\
\text { Reintegration and } \\
\text { Stabilization }\end{array}$ & $8-12$ weeks & $\begin{array}{l}\text { Uncertain, withdrawn, anxious, seemingly tired, } \\
\text { unpredictable, frustrated, resentful, avoidance, } \\
\text { denial, reluctance, physical ailments }\end{array}$ \\
\hline
\end{tabular}

- These times are guidelines. Depending on the length of the mission there may be more than one HLTA.

- Some stages may take longer than others and are dependent on other factors such as, how well parents are coping with the deployment.

- Rollercoaster effect might go back \& forth in stages.

•adapted from the Emotional Cycle of Deployment by Kathleen Vestal Logan

Figure 1. Deployment Cycle for Families (Egerton-Graham, 2009). Note that HTLA stands for Home Leave Travel Assistance.

\section{Ongoing Separation}

Although research suggests that attachment anxiety predicts reactions at the point of separation with a romantic partner, no research to date has examined the effects of attachment to 
coping during periods of prolonged separations with one's partner. Given that individuals high in attachment anxiety are said to experience chronic activation of the attachment system, it may be the case that such individuals will exhibit difficulties across the entire separation. In other words, individuals high in attachment anxiety may never effectively adjust to their partner's separation and experience emotional, psychological, and physiological difficulties as a result relative to low anxiety individuals. However, as outlined by Simpson and Rholes (1994) and further reiterated by Campbell and Marshall (2010), attachment dimensions are interactive in nature and are most relevant in contexts signalling attachment concerns. In particular, individuals high in attachment anxiety do not always show relationship difficulties in more neutral contexts. Therefore, it could also be argued that I should only really see the relevance of attachment dimensions, particularly anxiety, during the more stressful and transitional periods of separation and reunion. These transitional periods (such as stages 2 and 3 of the separation) are typically fraught with more stress for the couple, which should make internal working models more salient. In particular, as a result of heightened activation of the attachment system during these periods, individuals high in attachment anxiety might exhibit less effective emotion regulation leading to emotional and psychological difficulties, construe their partner's behaviour more negatively, and be less confident in their relationships relative to those low in attachment anxiety. Consistent with the logic stated above, I predicted that attachment anxiety would be related to greater distress during the prolonged separation given their chronic activation of the attachment system, but that this distress would be most pronounced during the transitional stages (closer to the initial separation and during the reunion period). On the other hand, in line with the separation phase, I predicted that attachment avoidance would be less predictive of self-reported distress during the prolonged separation as highly avoidant individuals tend to engage in 
distancing behaviours when stressed and require less comfort and support from attachment figures. In fact, attachment avoidance could be associated with enhanced well-being during transition stages.

I also predicted that highly anxious individuals would report more unrealistically optimistic expectations of their partners return across the prolonged separation, relative to less anxious individuals. This would be consistent with findings that individuals higher in attachment anxiety report being more dependent on their partners for validation and support in general, regardless of the context (e.g., Davilla, 2001; Wei, Mallinckrodt, Larson, \& Zakalik, 2005). If individuals high in attachment anxiety are generally more in need of reassurance and validation, regardless of whether their attachments are being threatened, they might be more prone to idealizing their future reunion at all points during the separation period, such as fantasizing about what the reunion period will be like. In other words, being more reliant on their partners for validation and reassurance, and experiencing greater separation distress, should lead anxious individuals to perceive the reunion phase more positively (e.g., "Everything will be better once he's home") leading them to be less realistic about what the reunion phase might ultimately be like.

Group A participants are also being asked to complete a final questionnaire shortly after they are reunited with their partners to assess functioning during the reunion phase similar to Group B. I expect similar findings with this group; because the reunion phase is a transitional period, there should be increased activation of attachment systems during this time and the increased relevance of attachment dimensions in predicting individual and relationship functioning. In particular, individuals high in attachment anxiety might be prone to experiencing emotional difficulties, lower quality partner interactions, decreased relationship satisfaction, and 
some difficulties providing emotional support to one's partner (Collins \& Feeney, 2000).

Individuals high in attachment avoidance will be less prone to report emotional difficulties, but will report more negative partner perceptions, lower quality partner interactions, decreased relationship satisfaction, and more discomfort in seeking emotional support from one's partner (Collins \& Read, 1990).

\section{Reunion}

Attachment anxiety seems to be related to greater fluctuations in daily interactions with one's partner such that individuals high in attachment anxiety seem to react more positively to positive events, and more negatively to negative events, relative to those low in anxiety (Campbell, Simpson, Boldry, \& Kashy, 2005; Tidwell, Reis, \& Shaver, 1996). Therefore, to be able to predict how attachment anxiety might relate to reactions to the reunion phase (more positive or negative reactions), one needs to first understand whether the reunion phase is construed as a (mostly) positive or negative event, or whether the reunion phase is more multifaceted with both negative and positive instances of intrapersonal coping, relationship interactions, and relationship perceptions. Some research suggests that the reunion (or postdeployment) phase is often identified by serving members as the "most stressful period" of the deployment cycle with the three most stressful issues being difficulty re-establishing intimacy with romantic partners, grieving the loss of independence and freedom, and the acceptance of new family routines (e.g., De Burgh et al. 2011). However, The Canadian Forces Deployment Handbook, informed by an empirically-derived model by Logan (1987), does describe the reunion as being a multifaceted experience, characterized by periods of positive intrapersonal and relationship functioning, and periods of negative intrapersonal and relationship functioning. According to this handbook, in the first couple of weeks (0-2 weeks' post reunion) most couples 
experience 'Elation', a second honeymoon period filled with mostly positive emotions including elation, happiness, and excitation. However, some spouses do report more negative emotional states including impulsivity, and nervousness. If individuals high in attachment anxiety are dependent on their partners for emotional comfort, reassurance, and validation, experience increased distress in response to partner separations, and desire greater levels of closeness in their relationships, it could be argued that those high in attachment anxiety should respond more positively to this honeymoon period. Such individuals might report feeling more emotionally connected to their partners, more satisfied in their relationships, and experience improved emotional well-being relative to those lower in attachment anxiety. On the other hand, highly avoidant individuals may be less satisfied with their partners' behaviour during the Elation period as they learn to cope with their partners being physically closer. Having one's partner home would naturally increase levels of intimacy in the relationship making avoidant individuals uncomfortable and might potentially lead to conflict and more unpleasant partner interactions. This would be in line with work by Tidwell, Reis, and Shaver (1996) that found that avoidant individuals reported less intimacy, enjoyment and positive emotions with their partners, and that avoidant individuals would structure social activities in ways that minimize intimacy and closeness. In other words, avoidant individuals avoid intimacy-building opportunities within their relationships, especially when they are stressed.

Couples then enter the 'Reintegration' (weeks 2-6) and 'Renegotiation' (weeks 6-12) phases which are characterized by greater reactivity or increased stress and decreased coping ability. Common emotions experienced during these phases include frustration, unpredictability, withdrawal, anxiety, physical ailments and fatigue. With many spouses reporting difficulties during these periods, it may be the case that these problems may be amplified for those high in 
attachment anxiety. There is some research supporting this notion, that attachment anxiety predicts a decline in marital functioning during major life transitions. For example, Rholes, Simpson, Campbell and Grich (2001) examined married couples becoming parents for the first time and found that anxious women perceived less support from their husbands during their pregnancy than less anxious women, and that anxious women's views of spousal support and marital satisfaction continued to worsen (so a negative linear trend) across the transition to parenthood. In explaining the findings by Rholes and colleagues (2001), Simpson and Rholes (2008) proposed that the negative outcomes experienced by high anxiety individuals during the transition to parenthood might be tied to their unrealistically optimistic views of their romantic partners and relationships. Specifically, anxious individuals may become frustrated and dissatisfied because they perceive that their partners are not living up to their expectations. Military spouses high in attachment anxiety might be prone to similar overly optimistic views about the post-deployment reunion phase, leading them to be disappointed when their partners' return does not live up to their expectations. As a result, highly anxious individuals may feel less close to their partners, less supported by them, less satisfied in their relationship, and they may not be able to cope as effectively as less anxious individuals. In contrast, individuals higher in attachment avoidance might show recovery from their initial difficulties with re-connection and might not report the difficulties that those lower in avoidance might.

Lastly, I will test mediation models to try to understand the relationships between attachment anxiety, in particular, and post-reunion difficulties. In line with findings by Rholes and colleagues (2001), I predicted that such difficulties might be driven by unrealistically optimistic expectations captured by reports that one's expectations of the reunion period have not been met. 


\section{Large-Scale Survey}

This survey was broad in scope, capturing not only how spouses have been impacted by military experiences personally (their own psychological and physical well-being), and in terms of their interpersonal relationships (particularly with their military spouse), but also how they are presently coping intrapersonally, as well as their perceptions of their relationship functioning and success. This survey also captured details surrounding deployments currently or recently experienced including time separated or reunited with a spouse due to a deployment. This information allowed me to test how deployment status (separated or reunited) moderated the effects of attachment orientations on intrapersonal and relationship functioning. In line with the previous two phases, I predicted that attachment anxiety and avoidance would be related to indices of intrapersonal and relationship functioning. In particular, I predicted attachment anxiety to be related to reports that one's partner's military experiences such as deployments and postings has had a more negative impact on the quality of their relationships, that various military experiences will be perceived as significantly more stressful relative to those lower in attachment anxiety. This would be in line with the findings of Fraley and Shaver (1998) and Mayseless and colleagues (1996) that those with anxious attachment report greater distress with experienced and imagined partner separations.

In line with phases one and two, I also predicted that the effects of attachment orientations on perceptions of intrapersonal and interpersonal functioning might be moderated by the presence or absence of an attachment-related threat (i.e., the deployment status of the partner). Specifically, the relationship between attachment anxiety and intrapersonal and relationship difficulties might be most pronounced for spouses who have been recently separated from their partners, or reunited with their partners, due to a deployment. With respect to 
attachment avoidance, I anticipate that the link between attachment avoidance and interpersonal difficulties might not vary as strongly as a function of deployment status, with the assumption that highly avoidant individuals report less distress upon experiencing attachment-related threats. On the other hand, as previously mentioned, it is possible to see some breakdowns in coping capacity and emotional well-being for highly avoidant spouses if military separations are perceived to be high-stress experiences for these individuals in line with findings by Mikulincer and Florian (1998).

Although the other two phases were assessed within a similar context across participants (similar deployment experience for most participants), which provides greater control over the effects of individual differences and extraneous variables on our key measures, the third phase of the project provides a broader view on the relationships among attachment dimensions and the impact of military experiences on relationship and interpersonal functioning with a larger sample than the first two phases. Most importantly, as a result of this increased power relative to the other phases, and with this being an independent design (not repeated measures) I was also able to more easily assess the trajectory of the effects of attachment dimensions on intrapersonal and relationship outcomes as a function of time separated, and time reunited. Finally, a number of measures relevant to demographics, individual differences, and relationship variables included in the survey (e.g., perceived partner support, relationship cohesion, relationship satisfaction), and adequate sample size, I was able to use mediation models to identity consistent and powerful mediators in the relationships between attachment orientations and intrapersonal and relationship outcomes.

In summary, although one initial study points to the relevance of adult attachment in spousal adjustment to deployment experiences, specifically the post-reunion period (Cafferty et 
al., 1994), methodological concerns and lack of more complex statistical analyses leave many questions unanswered. First, it is not clear what attachment dimensions specifically are related to problems in emotional and relationship adjustment during deployment phases as previous research did not assess the unique and interactive effects of attachment anxiety and attachment avoidance, but instead used the Hazan and Shaver categorical approach. Next, it is not clear from the current literature on attachment and long term separations and transition periods why particular attachment dimensions are related to such outcomes (what mechanisms can explain these relationships). Finally, we do not yet know when or how attachment dimensions are relevant in predicting outcomes across the deployment cycle (during the separation, reunion, and what trajectory?). These are the goals of my dissertation. 


\section{Chapter 2}

\section{Phase One - Separation Group}

The main purpose of this study was to assess the unique and interactive effects of attachment anxiety and avoidance on perceptions of individual and relationship functioning during a major partner separation due to deployment. In line with findings from Fraley and Shaver (1998) and Cafferty and colleagues (1994), I predicted that, given their strong desires for closeness and intimacy and their reliance on attachment figures for emotional regulation and support, individuals higher in attachment anxiety would report greater difficulties during the separation relative to individuals lower in attachment anxiety. Specifically, I predicted that attachment anxiety would be related to higher levels of distress, negative affect, decreased coping capacity relative to those lower in attachment anxiety. Further, I predicted that attachment anxiety would be related to more negative relationship perceptions during the separation in line with previous research (e.g., Collins \& Read, 1990).

In addition, I predicted that attachment avoidance should be associated with deactivating strategies in response to environmental threats such that attachment avoidance should not be associated with reported distress or indices of well-being. Alternatively, it is possible to see some breakdowns in coping capacity and emotional well-being for highly avoidant spouses if military separations are perceived to be high-stress experiences (Mikulincer \& Florian (1998).

I also predicted that the effects of attachment dimensions might be amplified as a function of stress experienced as they are interactive in nature (Simpson \& Rholes, 1994; Campbell \& Marshall, 2010), and are most relevant in contexts signalling attachment concerns. Therefore, we should see the greatest relevance of attachment dimensions, particularly anxiety, during the more stressful and transitional periods of a separation (i.e., stages 2 and 3). Further, 
perceptions of how stressful the deployment separation is also depends on one's experience with deployments. Indeed, the negative effects of deployment separations seem to be amplified for those who are experiencing their first deployment (Padden, Connors, \& Agazio, 2011; Frankel, Snowden, \& Nelson, 1993). Therefore, I expect that the effects of attachment dimensions might be moderated by previous deployment status.

\section{Method}

\section{Participants and Procedure}

DGMPRA mailed surveys to civilian spouses/partners of those who were Regular Force (not Augmentees or Reservists, or dual-service couples, and who had a listed home address as surveys were administered using Canada Post. In total, surveys were sent to 373 spouses of CAF Regular Force personnel who had been deployed. At the end of the survey, spouses were given the option to be entered into one of five draws for $\$ 100$. The remuneration was provided $100 \%$ by external researchers at Queen's University. A total of 85 spouses completed the survey, a response rate of $23 \%$, which is typical for military samples ${ }^{2}$. The spouses were all women between 23-51 years of age $(M=34.52, S D=7.70)$, and had been married or in a common-law relationship with their partner ${ }^{3}$ for an average of 6.80 years $(S D=6.54$ years $)$. Spouses' partners were mostly in the Canadian Army (CA) (78\%, 9\% Royal Canadian Air Force (RCAF), $6 \%$ Royal Canadian Navy (RCN), 7\% undisclosed) and had recently deployed to Afghanistan (96\%). For many spouses, this was not the first time they had experienced a military deployment (53\%), and at the time of completing the survey had been separated from their partners for an average of

\footnotetext{
${ }^{2}$ Researchers internal and external to the Department of National Defence (e.g., Newell, Rosenfeld, Harris, \& Hindelang, 2004; Lamerson, 2001) have reported decreasing response rates for military samples since the 1980s with recent samples hovering around $30 \%$ compliance.

${ }^{3}$ All partners were men between 20-50 years of age and were 6 months older than the female spouses on average $(M$ $=35.01, S D=7.19$ ). An age difference of less than 2 years is typical for married samples (e.g., Meltzer, McNulty, Jackson, \& Karney, 2014; Neff \& Geers, 2014).
} 
84.81 days $(S D=21.03 \text {, range }=22-189 \text { days })^{4}$. Most of the women indicated that they had children (69\%), most were employed (70\%) and, of those employed, $88 \%$ worked outside the home.

\section{Measures}

Attachment Anxiety

The Experiences in Close Relationships Scale- Revised (ECR-R: Fraley, Waller, \& Brennan, 2000; see Appendix A) assesses an individual's level of attachment anxiety and avoidance in close relationships. This 36 -item questionnaire includes 18 items that measure anxiety (e.g., "I often worry that my partner doesn't really love me") and 18 items that measure avoidance (e.g., "I prefer not to show my partner how I feel deep down”). Responses are indicated on a scale from 1 (Strongly Disagree) to 7 (Strongly Agree) and total scores for each dimension are calculated using the mean of the respective 18 items with higher scores indicating higher levels of attachment anxiety and avoidance (see Table 1 for descriptive statistics). In this sample, scale scores for both anxiety (Cronbach's alpha $=.88)$ and avoidance (Cronbach's alpha $=.88)$ were found to be reliable and were correlated, $r(85)=.59, p<.001$.

\section{Self-Esteem}

The Rosenberg Self Esteem Scale (RSE: Rosenberg, 1965; see Appendix B) contains 10 items that measure self-esteem (e.g., “On the whole, I am satisfied with myself”). Responses are indicated on a scale from 1 (Strongly Disagree) to 7 (Strongly Agree) and total scores for selfesteem are calculated using the mean of all 10 items (after reverse coding negatively phrased items). Higher scores indicate higher levels of self-esteem (see Table 1 for descriptive

\footnotetext{
${ }^{4}$ It should be noted that number of days separated was not significantly correlated with attachment anxiety nor avoidance ( $r \mathrm{~s}=.13$ and .07 respectively).
} 
statistics). The scale scores for this measure of self-esteem were found to be reliable (Cronbach's alpha $=.90)$.

\section{Relationship Satisfaction}

Relationship satisfaction was assessed using the Relationship Assessment Scale (RAS:

Hendrick, 1988; see Appendix C). This scale has 6 items (e.g., "How much do you love your partner?") and I included two additional items for added reliability ("How much do you trust your spouse?" and "How often do you wish you hadn't gotten into this relationship?)".

Responses are indicated on a scale from 1 (Strongly Disagree) to 7 (Strongly Agree) and total scores for relationship satisfaction are calculated using the mean of all eight items (after reverse coding negatively phrased items). Higher scores indicate greater relationship satisfaction (see Table 1 for descriptive statistics). With this sample, the scale scores for relationship satisfaction were found to be reliable (Cronbach's alpha $=.86$ ).

State Anxiety

I used the State Trait Anxiety Inventory (STAI: Spielberger, Gorsuch, Lushene, Vagg, \& Jacobs, 1970; see Appendix D) to measure the extent to which individuals are experiencing state (or current levels of) anxiety. Respondents are asked to indicate the appropriate response for each of the 20 statements (e.g., "I feel tense") on a scale from 1 (not at all) to 4 (very much so). A total state anxiety score is calculated using the average of all 20 items once negatively worded items are reverse scored, with higher scores indicating higher levels of state anxiety (see Table 1 for descriptive statistics). State anxiety scale scores were found to be reliable (Cronbach's alpha $=.92)$. 


\section{Positive and Negative Affect}

The Positive and Negative Affect Schedule (PANAS: Watson, Clark, \& Tellegen, 1988;

see Appendix E) was used to measure current experiences of positive and negative affect. There are 10 items that measure positive affect (e.g., "excited") and 10 items that measure negative affect (e.g., "irritable"). Respondents indicate the extent to which they are experiencing each item on a scale from 1 (not at all) to 5 (extremely). Total scores for positive and negative affect are calculated using the mean of the respective 10 items, with higher scores reflecting greater positive and negative affect respectively (see Table 1 for descriptive statistics). For this sample, positive affect scale scores (Cronbach's alpha $=.87)$ and negative affect scale scores $($ Cronbach's alpha $=.87)$ were reliable.

\section{Relationship Quality}

Relationship quality was assessed using a measure developed by MacDonald and Ross (1999; see Appendix F) and includes the following subscales: relationship harmony (12 items), relationship importance (single item), ease of finding an alternative partner (single item), predicting a positive future for the relationship (three items), negative future for the relationship (three items), and feeling as though relationship events are within one's control (single item). Responses to each item are indicated on a scale from 1 (Strongly Disagree) to 7 (Strongly Agree).

In order to determine whether I could uncover a fewer number of latent variables that could account for bivariate correlations among these items ( $r$ s ranging from .003 to .756 ), instead of performing a confirmatory factor analysis given that there was no strong theoretical foundation behind the six factors discovered by MacDonald and Ross. I conducted exploratory 
factor analyses (EFA) in accordance with procedures recommended by Fabrigar and Wegener (2012).

In accordance with recommendations by Fabrigar and Wegener (2012), I then conducted an EFA using Maximum Likelihood (ML) as the fitting model, relative to Principal Axis Factoring, given the additional information that it provides (tests of fit, confidence intervals, and statistical tests of parameters). In order to determine the number of factors present amongst these 21 variables, I first used the scree test, by examining the scree plot based on eigenvalues from the reduced matrix (see Figure 2). Although the eigenvalue-greater-than-one rule (or Kaisan criterion) is probably one of the more widely used procedures for assessing the number of common factors, it's meant to be used on eigenvalues from the unreduced correlation matrix, and is therefore inappropriate in the context of common factor analysis (see Fabrigar \& Wegener, 2013). Using this method, the appropriate number of common factors corresponds to the number of eigenvalues prior to the last major drop (Fabrigar \& Wegener, 2012). According this plot, there is a good case for the presence of 1 factor, with there being a substantial drop in variance in explained among variables after the first factor (from 7.04 to 1.28). However, one could also argue for a three-factor solution, with there being another visible drop in the eigenvalues after the third factor (from 1.05 to .64).

As a secondary test for determining the appropriate number of common factors, I performed a parallel analysis which compares the eigenvalues from the sample data with eigenvalues obtained from random data. If the eigenvalues from the sample data are larger than those produced randomly, this indicates the likely presence of 'real' common factors. I used the following parameters: $\mathrm{N}=85$, number of variables $=21$, number of datasets $=100$, and confidence interval $=.95$. According to this analysis, only one factor had an eigenvalue greater 
than those produced by random data, either by the mean or $95^{\text {th }}$ percentile values. Therefore, according to this analysis, the one factor solution is more appropriate.

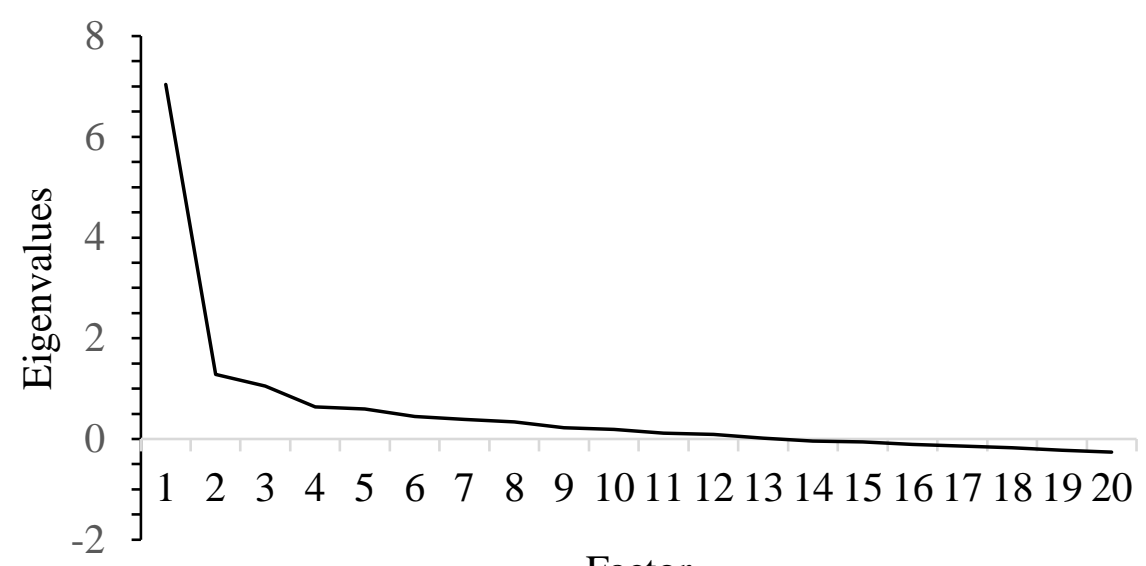

Factor

Figure 2. Scree plot of the reduced correlation matrix for relationship quality items (Phase One separation).

The final method I used to determine the appropriate number of factors was computing the root mean square error estimates for one, two, and three factor models. Using the RMSEA as a fit index is recommended over the chi-square likelihood ratio test statistic, as the likelihood ratio test statistic has a number of drawbacks. For example, the likelihood ratio test statistic can make it likely to produce models that are overfactored with large samples, and underfactored with small samples (see Fabrigar \& Wegener, 2012). As a guideline, an RMSEA from .05-.08 indicates an acceptable fit, with RMSEAs below .05 indicating a close fit. Accordingly, all three models indicated an acceptable fit for the data, (one-factor RMSEA $=.077$, two-factor RMSEA $=.065$, three-factor RMSEA $=.052$ ). While the three-factor model certainly showed the best fit index relative to the previous two models, all were in the acceptable range. In order to better determine whether a three-factor model was appropriate, I next examined the interpretability of the factor loadings. 
To interpret the three factors, I conducted a ML EFA with an oblique rotation given that there is no substantive reason to assume that these factors are orthogonal to one another. I first examined the communalities, which ranged from .07-.99, and only six communalities less than .30 , suggesting that the factors explained a good proportion of the variance in each of the items. Further, some factors correlated with one another (factor correlation coefficients ranging from .20 to .37 ) suggesting that an oblique rotation was appropriate.

The first factor explained 33.5\% of the variance across measures (Eigenvalue $=7.04$ ), was labelled wanting change (two items: e.g., "I do not like aspects of my partner's personality"), and reflects disliking aspects of a partner and desiring change in a partner. Scores for these items were calculated taking the mean of the two items. The scale scores were found to be reliable (Cronbach's $\alpha=.77)$.

The second factor explained 6\% of the variance amongst items (Eigenvalue $=1.28$ ), was labelled relationship harmony (10 items: e.g., "My partner and I have similar goals"), and reflects feeling as though one is a good 'match' with their partner, that the couple communicates well, and feeling valued and emotionally supported by one's partner. Scores for relationship harmony were calculated by taking the mean of the respective items with higher scores indicating greater relationship harmony. The scale scores were found to be reliable (Cronbach's $\alpha=.90)$ and no items would improve reliability if deleted.

The third factor accounted for $5 \%$ of the variance across measures (Eigenvalue $=1.05$ ), was labelled commitment and closeness (six items: e.g., "I never tire of my spouse's company, no matter how much time we spend together"), and reflects feeling attracted to one's partner, never tiring of their company, and feeling sure about continuing the relationship. Scores for commitment and closeness were calculated by taking the mean of respective items after reverse 
coding necessary items with higher scores indicating more partner attraction. The scale scores' reliability was questionable (Cronbach's $\alpha=.66$ ) and no items would improve reliability if deleted. I decided to maintain this subscale instead of treating these variables as separate subscales, given that the estimate was closer to the acceptable cut off than questionable cut-off, and for model parsimony.

In addition to these factors, there were three items that did not load highly (loadings > .30) onto any factors, and one item that loaded highly on more than one factor. Therefore, the following three items were treated as separate variables: "Over time, it is likely that I will discover areas in which my needs conflict with those of my partner" (conflict over time), "Whether this relationship continues depends primarily on factors within my control" (relationship within control), and "I could get by without a romantic relationship" (get by without). Descriptive statistics for all relationship quality factors and items can be found in Table 1.

\section{Coping Capacity}

I created a total of 14 items (see Appendix G) that captured coping capacity or general well-being with facets such as emotional (e.g., "I am feeling upbeat"), physical (e.g., "I have been sleeping well”), social (e.g., "I feel good about my social life"), and practical (e.g., "I can manage the ups and downs of everyday life") functioning. Respondents indicated their agreement with these items on a scale from 1(Strongly Disagree) to 7(Strongly Agree). I performed an EFA to see if I could uncover a fewer number of latent variables that could account for bivariate correlations among these items ( $r$ s ranging from .008 to .563 ) using ML as the fitting model and other procedures outlined by Fabrigar and Wegener (2012), similar to the relationship quality scale. 
In order to determine the number of factors present amongst these 14 variables, I used the scree test, by examining the scree plot based on eigenvalues from the reduced matrix (see Figure 3). According to this plot, there is a good case for the presence of one factor, with there being a major drop in the eigenvalue after the first factor (from 4.97 to 0.79). However, one could also argue for the presence of four factors, with the last major and visible drop in variance explained occurring after the fourth eigenvalue (from .45 to .16 ).

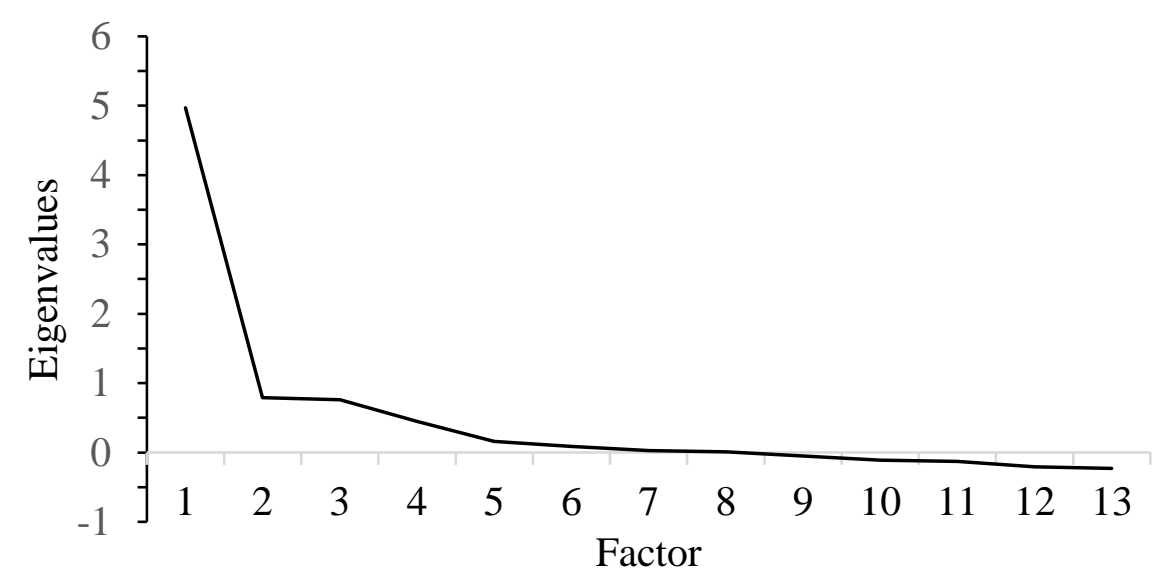

Figure 3. Scree plot of the reduced correlation matrix for coping capacity items (Phase One separation).

As a secondary test for determining the appropriate number of common factors, I performed a parallel analysis. I used the following parameters: $\mathrm{N}=85$, number of variables $=14$, number of datasets $=100$, and confidence interval $=.95$. According to this analysis, four factors had an eigenvalue greater than those produced by random data, either by the mean or $95^{\text {th }}$ percentile values. Therefore, according to this analysis, the four-factor solution seems appropriate.

The final method I used to determine the appropriate number of factors was assessing model fit using the root mean square error estimates for one, two, three, and four factor models. 
The one-factor and two-factor models showed an acceptable fit for the data $(\mathrm{RMSEAs}=.075$ and .058 respectively). However, the RMSEAs for the three and four factor models were zero, because the chi-square values were less than the degrees of freedom for these models. Therefore, while the scree test and parallel analysis indicated that four factors might be appropriate, the model fit index was not consistent with this interpretation. In order to better determine whether a four-factor model was appropriate, I next examined the interpretability of the factor loadings.

To interpret the three factors, I conducted a ML EFA with an oblique rotation given that there is no substantive reason to assume that these factors are orthogonal to one another. I first examined the communalities, which ranged from .31-.72, suggesting that the factors explained a good proportion of the variance in each of the items. Further, some factors correlated with one another (factor correlation coefficients ranging from .15 to -.54) suggesting that an oblique rotation was appropriate.

The first factor explained $35.5 \%$ of the variance across measures (Eigenvalue $=4.97$, was labelled physical well-being (five items: e.g., "I am feeling healthy"), and reflects feeling energetic, healthy, well rested, and socially active. Scores for this factor were calculated by taking the mean of the respective items, with higher scores reflecting better physical well-being. The scale scores were found to have good reliability (Cronbach's $\alpha=.81$ ).

The second factor explained $5.6 \%$ of the variance amongst items (Eigenvalue $=0.79$ ), was labelled emotional well-being (three items: e.g., "I feel overwhelmed"), and reflects feeling depressed, anxious, and lonely. Scores for emotional well-being were calculated by reverse scoring all items and then calculating the mean of these items such that higher scores indicated better emotional well-being. The reliability for scale scores was acceptable (Cronbach's $\alpha=.65$ ). 
The third factor accounted for 5.4\% of the variance across measures (Eigenvalue $=0.76$ ), and was comprised of (two items: "I feel supported" and "I am satisfied with my marriage"). The reliability of the scale scores was poor (Cronbach's $\alpha=.48$ ). For this reason, the items should be treated as separate measures. However, given that I already had a measure of relationship satisfaction, I decided to drop the marital satisfaction measure, and retain the perceived social support item.

The fourth factor accounted for $3.2 \%$ of variance in measures (Eigenvalue $=0.45)$. There were two items that loaded highly on this factor, and another two items that loaded highly on this factor, and one other factor (cross-loadings). Conceptually, the items with cross-loadings seemed to fit with the other items on factor four, capturing the extent to which individuals felt like they were coping with daily life. For this reason, and to make the model more parsimonious, I decided to retain the fourth factor with four items, and labelled it practical well-being (four items: e.g., "I can manage the ups and downs of everyday life"). Specifically, this factor reflects feeling good about one's financial situation, completely managing daily life, and feeling good about oneself. Scores for this factor were calculated by taking the mean of the four items with higher scores indicating better practical well-being. The scale scores were reliable (Cronbach's $\alpha$ $=.77)$. Descriptive statistics for all coping capacity factors can be found in Table 1 . 
Table 1

Descriptive Statistics for Separation Measures

\begin{tabular}{|c|c|c|c|}
\hline & & $M$ & $S D$ \\
\hline Attachment anxiety $^{1}$ & & 2.27 & 0.90 \\
\hline Attachment avoidance $^{1}$ & & 2.07 & 0.77 \\
\hline Self-esteem ${ }^{1}$ & & 5.81 & 0.93 \\
\hline Relationship satisfaction $^{1}$ & & 6.36 & 0.57 \\
\hline State anxiety ${ }^{2}$ & & 1.70 & 0.49 \\
\hline Positive affect ${ }^{3}$ & & 3.50 & 0.71 \\
\hline Negative affect ${ }^{3}$ & & 1.52 & 0.55 \\
\hline \multirow[t]{6}{*}{ Relationship quality $^{1}$} & Wanting change factor & 3.75 & 1.59 \\
\hline & Relationship harmony factor & 6.25 & 0.74 \\
\hline & Commitment closeness factor & 6.05 & 0.75 \\
\hline & Conflict over time & 3.16 & 1.72 \\
\hline & Relationship within control & 4.04 & 2.00 \\
\hline & Get by without & 3.95 & 1.92 \\
\hline \multirow[t]{4}{*}{ Coping capacity $^{1}$} & Physical well-being & 5.23 & 1.17 \\
\hline & Emotional well-being & 4.31 & 1.36 \\
\hline & Perceived social support & 6.19 & 0.72 \\
\hline & Practical well-being & 5.70 & 0.93 \\
\hline \multicolumn{4}{|c|}{${ }^{1}$ Response range $=1($ Strongly Disagree $)$ to 7 (Strongly Agree $)$} \\
\hline \multicolumn{4}{|c|}{${ }^{2}$ Response range $=1$ (not at all) to 4 (very much so) } \\
\hline \multicolumn{4}{|c|}{${ }^{3}$ Response range $=1$ (not at all) to 5 (extremely) } \\
\hline
\end{tabular}

\section{Results}

First, I was interested in assessing the unique and interactive effects of attachment anxiety and avoidance on perceptions of individual and relationship functioning during a major partner separation due to deployment. To do so, I conducted a series of multiple linear regressions with anxiety, avoidance, and their interaction term as predictors on the following dependent variables: positive and negative affect, relationship satisfaction, relationship quality (relationship harmony, partner attraction, wanting change, closer with events, satisfied over time, conflict over time, partner gets along, find another partner, get by without, relationship within control), and coping capacity (physical well-being, emotional well-being, social/marital support, practical well-being). I tested for the presence of potential confounds that have been reported in prior work on attachment anxiety including self-esteem (e.g., Collins \& Read, 1990; 
Bartholomew \& Horowitz, 1991), relationship length (e.g., Duemmler \& Kobak, 2001; Fraley \& Shaver, 1998), and whether or not the spouse had children (e.g., Medway, Davis, Cafferty, Chappell, \& O'Hearn, 1995). After conducting bivariate correlations between attachment anxiety and all potential covariates, the only significant relationship that emerged was that between attachment anxiety and self-esteem, $(r(83)=-.53, p<.001)$. As a result, self-esteem was included in all analyses as a covariate. Following the recommendations of Aiken and West (1991), scores for anxiety, avoidance, and self-esteem were mean centered in order to reduce multicollinearity ${ }^{5}$. Further, the anxiety and avoidance interaction term was calculated using the mean centered scores.

Conducting regression analyses with 15 dependent variables, and three predictors can raise concerns regarding inflation of alpha rates. Specifically, by chance I could find 2 significant effects. Although this is a real concern, I also did not have the statistical power to compensate for this type I error inflation without also compromising power and increases in type II error. Specifically, to maintain a .001 alpha rate $(.05 / 45)$, and power at .90 , with three predictors, I would need a minimum of 145 participants. For this reason, I decided to not implement an alpha correction and maintain the .05 alpha criterion. Therefore, any significant findings should be interpreted with caution.

There were no significant interactions between attachment anxiety and avoidance on any of the measures, but there were significant main effects. Specifically, attachment anxiety was positively associated with state anxiety $(B=.23, \beta=.46, t(80)=3.44, p=.001)$, and negatively associated with relationship satisfaction $(B=-.32, \beta=-.50, t(80)=-4.77, p<.001)$, relationship

\footnotetext{
${ }^{5}$ According to Aiken and West (1991), if first order variables are not centered, product terms can be highly correlated with the first order terms, which can lead to large standard errors for the lower order terms when included in regression analyses with higher order terms.
} 
harmony $(B=-.30, \beta=-.37, t(80)=-3.12 p=.003)$, emotional well-being $(B=-.62, \beta=-.41$

$t(80)=-3.24, p=.002)$, and perceived social support $(B=-.41, \beta=-.34, t(80)=-2.45, p=.016)$.

Attachment avoidance was negatively associated with relationship satisfaction $(B=-.28$, $\beta=-.38, t(80)=-3.76, p<.001)$, relationship harmony $(B=-.40, \beta=-.41, t(80)=-3.69, p<$ $.001)$, and commitment and closeness $(B=-.41, \beta=-.42, t(80)=-3.42, p=.001)$. Attachment avoidance was also positively associated with desires to change a partner's characteristics $(B=$ $.58, \beta=.28, t(80)=2.19, p=.032)$, feeling as though one could get by without a romantic relationship $(B=.77, \beta=.31, t(80)=2.27, p=.026)$, and emotional well-being $(B=.52, \beta=.29$, $t(80)=2.44, p=.017)$. In addition, attachment avoidance was marginally negatively related to positive affect, $B=-.24, \beta=-.25, t(80)=-1.88, p=.063$.

I also predicted that the effects of attachment dimensions might be amplified for those who are experiencing their first deployment. I tested this hypothesis, assessing whether the significant relationships between attachment anxiety and state anxiety, relationship satisfaction, relationship harmony, emotional well-being, and perceived social support are moderated by whether or not couples had experienced deployments before, by adding the (centered) previous deployment variable and the anxiety by previous deployment interaction term to the regression models for all four dependent measures. I indeed found a significant two-way interaction between anxiety and previous deployment status for relationship harmony, $B=.16, \beta=.20, t(78)$ $=2.22, p=.029$, but none of the other interaction coefficients approached significance $(p \mathrm{~s}$ between .12-.75). I decomposed this interaction using simple slopes analysis (Aiken \& West, 1991) in order to examine the effects of attachment anxiety on relationship harmony for those who were experiencing their first deployment versus those who were not experiencing their first deployment. For those who were experiencing a deployment for the first time, there was a 
significant negative relationship between attachment anxiety and relationship harmony, $B=-.46$, $\beta=-.56, t(78)=-3.88, p<.001$ (see Figure 4). However, for those who have experienced deployments before, there was no significant relationship between attachment anxiety and relationship harmony, $B=-.14, \beta=-.17, t(78)=-1.14, p=.260$.

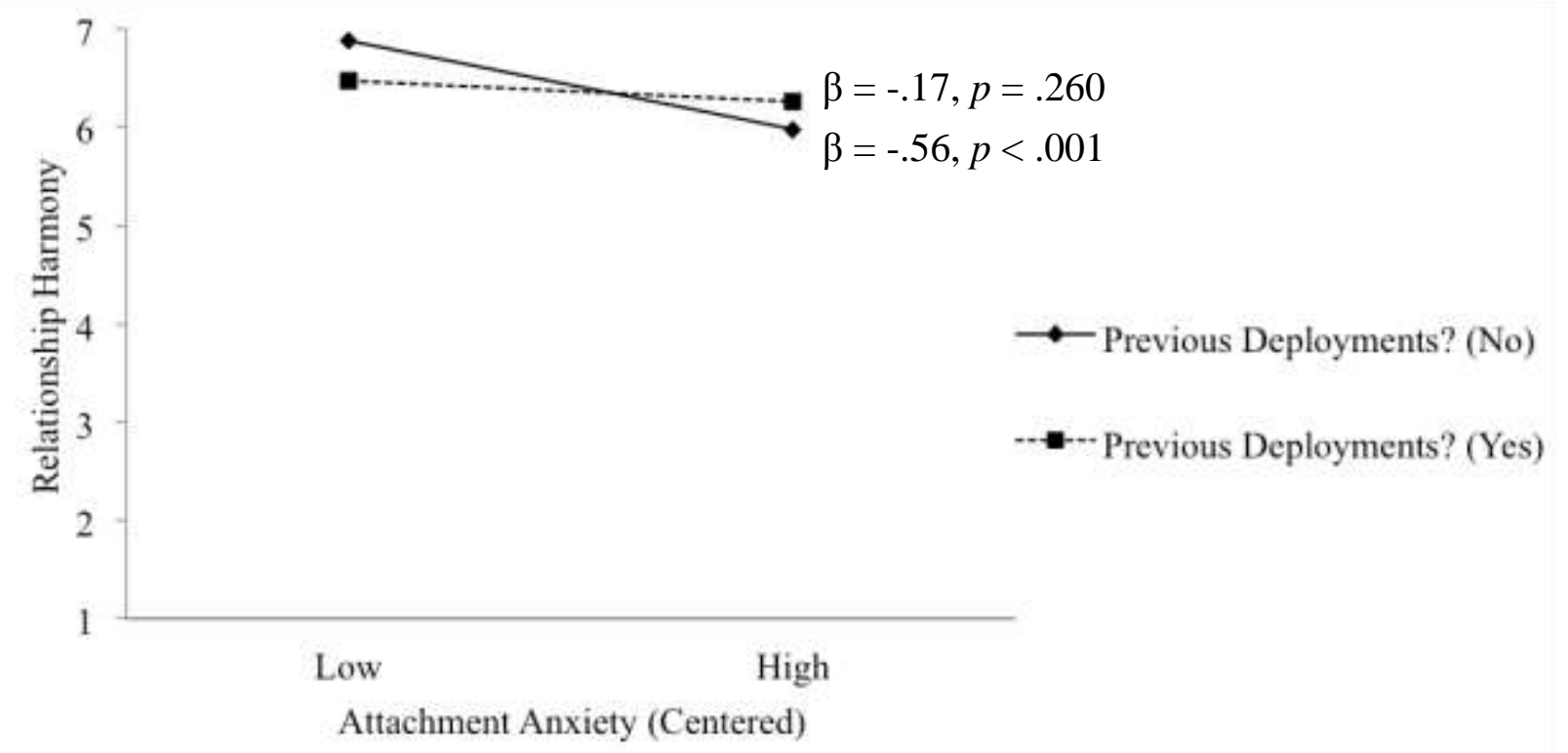

Figure 4. Interaction between attachment anxiety and previous deployment status on perceived relationship harmony during the deployment separation.

Next, I wanted to test whether the relationships between attachment anxiety and perceptions of intrapersonal functioning and relationship quality are moderated by the stage of the separation (stages 2 and 3 versus 4) which vary in the extent to which they are perceived as being stressful or difficult. Unfortunately, I only had 3 individuals who were in the 'reacting' stage 3, and no participants who were experiencing stage 2. However, I was still interested in assessing whether the effects of attachment dimensions might be moderated by the number of days separated, even if most participants are in the recovery and stabilization period (stage 4). In particular, I was interested in assessing whether the intrapersonal and relationship difficulties 
that highly anxious individuals are experiencing, relative to less anxious individuals, were exacerbated closer to or further from the initial separation. Similar to the above analysis, I ran multiple linear regressions with anxiety and avoidance (centered), the anxiety and avoidance interaction term, days separated (centered), and the anxiety by days separated interaction term as predictors on the five criterion variables similar to above (state anxiety, relationship satisfaction, relationship harmony, emotional well-being, social/marital support). First, I found a significant anxiety by days separated interaction for relationship satisfaction $(B=-.01, \beta=-.19, t(74)=-$ $2.11, p=.038)$, and relationship harmony $(B=-.01, \beta=-.26, t(74)=-2.71, p=.008) . \mathrm{I}$ decomposed these interactions using simple slopes analyses (Aiken \& West, 1991) to examine the effects of days separated on relationship satisfaction and harmony at low (-1 SD below the mean) versus high (+1 SD above the mean) levels of anxiety ${ }^{6}$.

At low levels of anxiety, there was no significant relationship between days separated and satisfaction $(B=.01, \beta=.15, t(74)=1.01, p=.316$; see Figure 5). However, at high levels of anxiety there was a significant negative relationship between days separated and satisfaction $(B=-.01, \beta=-.23, t(74)=-2.30, p=.024)$. Similarly, at low levels of attachment anxiety, the relationship between days separated and relationship harmony was not significant $(B=.01, \beta=$ $.19, t(74)=1.26, p=.213$; see Figure 6$)$. However, at high levels of anxiety there was a significant negative relationship between days separated and relationship harmony $(B=-.01, \beta=$ $-.38, t(74)=-3.63, p=.001)$.

\footnotetext{
${ }^{6}$ In reality, simple slopes for days reunited were calculated at -1 and +1 SD from the centered mean (so values equal to 64 days and 106 days separated, respectively). The decentered values are presented for better interpretability.
} 


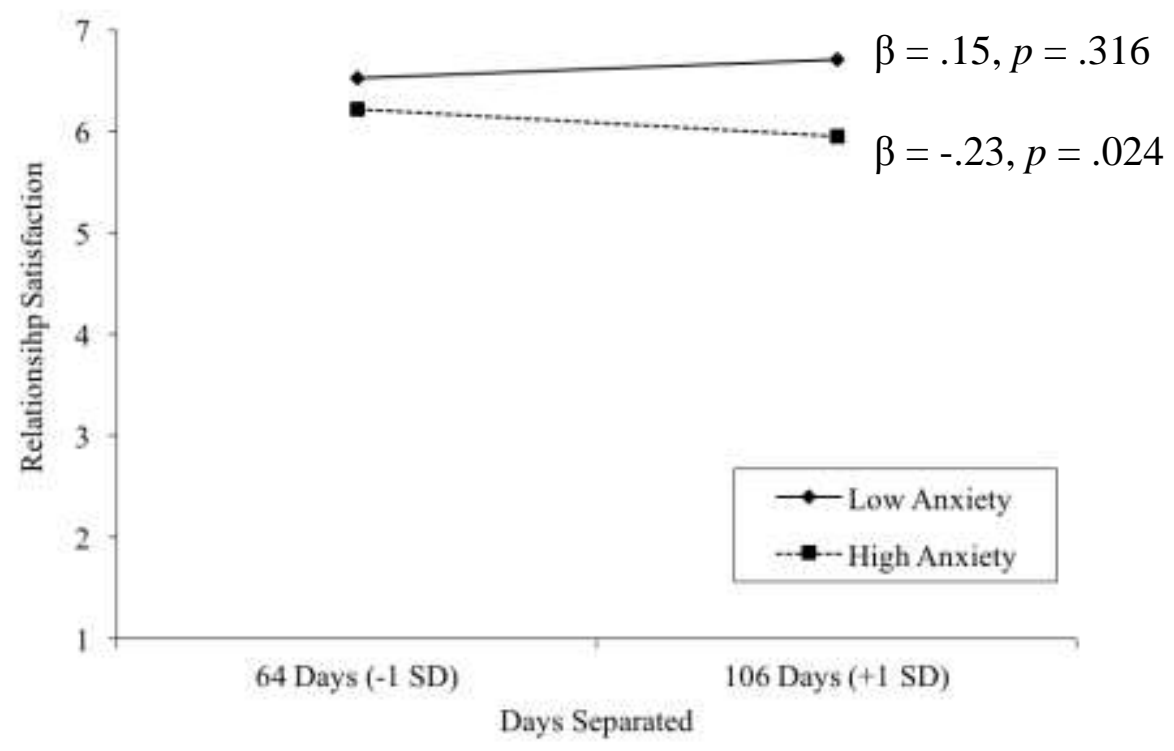

Figure 5. Interaction between attachment anxiety and number of days separated on relationship satisfaction during the deployment separation.

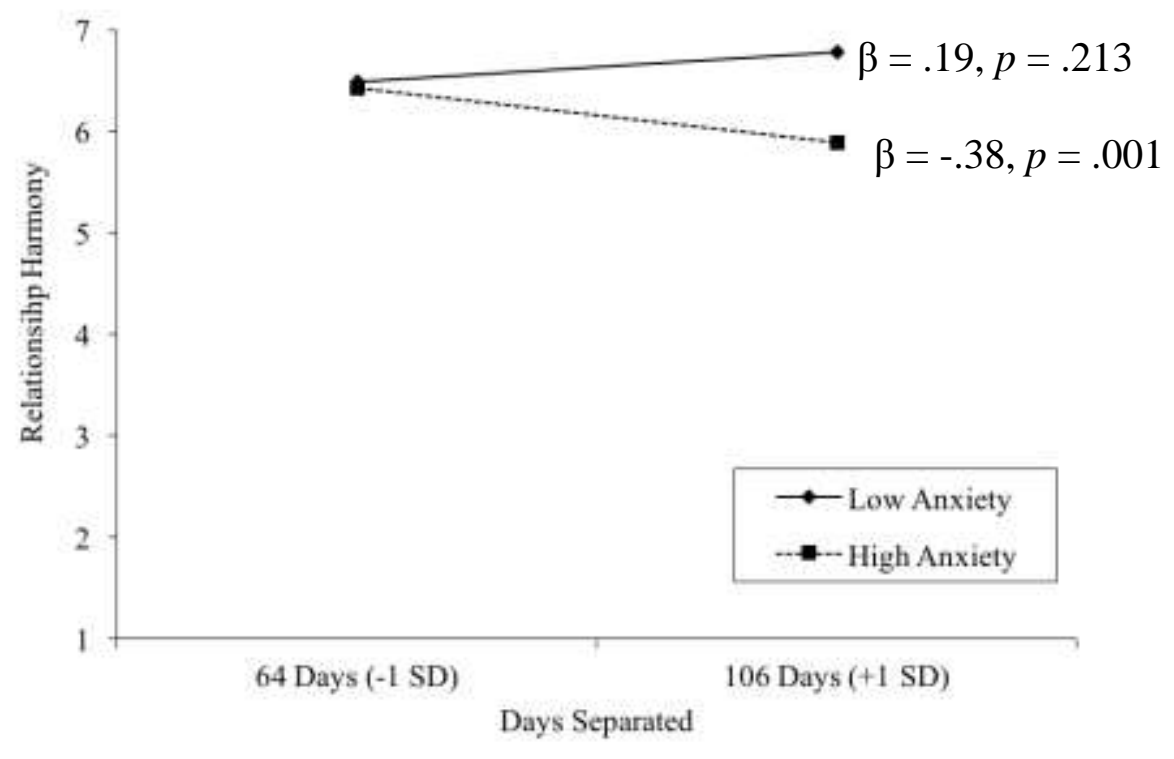

Figure 6. Interaction between attachment anxiety and number of days separated on perceived relationship harmony during the deployment separation. 


\section{Discussion}

Consistent with findings from Fraley and Shaver (1998) and Cafferty and colleagues (1994), I predicted that individuals higher in attachment anxiety would report greater difficulties during the separation relative to individuals lower in attachment. Further, I predicted that attachment anxiety would be related to more negative relationship perceptions (e.g., Collins \& Read, 1990). In line with my predictions, higher levels of attachment anxiety were associated with decreased emotional well-being and more negative relationship perceptions during the separation relative to lower levels of attachment anxiety. Specifically, attachment anxiety was associated with higher levels of state anxiety, negative affect, and emotional well-being, as well as less relationship satisfaction, less relationship harmony, and less perceived social support.

In addition, I predicted that attachment avoidance would be associated with deactivating strategies in response to environmental threats such that attachment avoidance and as such would not be associated with reported distress or compromised well-being. On the other hand, it is possible to see some breakdowns in coping capacity and emotional well-being for highly avoidant spouses if military separations are perceived to be high-stress experiences (Mikulincer \& Florian, 1998). Interestingly, and in line with the former theorizing, attachment avoidance was actually associated with increased emotional well-being, but related to more negative relationship perceptions compared to attachment anxiety. Specifically, attachment avoidance was associated with less relationship satisfaction, less relationship harmony, less commitment and closeness, a stronger desire to change a partner's characteristics, and feeling more strongly that one could get by without a romantic relationship.

I also predicted that the effects of attachment dimensions might be exacerbated by factors that might amplify the magnitude of how stressful the deployment is perceived to be, or during 
periods of the deployment that involve increased attachment concerns, given that attachment orientations and attachment threats are interactive in nature (Simpson \& Rholes, 1994; Campbell \& Marshall, 2010). Therefore, I predicted that the effects of attachment dimensions on functioning during a deployment might to be amplified for those who are experiencing their first deployment (Padden, Connors, \& Agazio, 2011; Frankel, Snowden, \& Nelson, 1993), and during the more stressful periods of a separation (i.e., stages 2 and 3). I did find evidence to suggest that the effects of attachment anxiety on relationship perceptions during a deployment separation are exacerbated for those who are experiencing a deployment for the first time. Specifically, I found that for those who were experiencing a deployment for the first time, there was a significant negative relationship between attachment anxiety and relationship harmony. However, for those who have experienced deployments before, there was no significant relationship between attachment anxiety and relationship harmony. In other words, attachment anxiety is more predictive of relationship perceptions during first experiences of a separation, and is less predictive of relationship perceptions when a couple has had experience with previous deployment separations. Previous deployment status did not, however, moderate the effects of attachment avoidance on intrapersonal or relationship outcomes.

Following this logic, I predicted that the effects of attachment orientations might be exacerbated during stages 2 (detachment and withdrawal: 1-3 weeks before the separation) and 3 (emotional disorganization: weeks 1-6 after the separation) relative to stage 4 (recovery and stabilization: weeks 6-12 after separation). Unfortunately, I did not have enough responses at stages 2 or 3 to test these predictions, with none completing the survey prior to the separation, and only 3 who completed the survey during stage 2 . However, I was still able to test linear effects of time separated although the anchors (representing 1 standard deviation below and 
above the mean) of comparison were technically both in the recovery and stabilization period or stage 3 (weeks 9 and 15 respectively). Specifically, my findings suggest that the trajectory of relationship perceptions across the deployment separation might not be the same for those low versus high in attachment anxiety. For those lower in attachment anxiety, there was no relationship between days separated and relationship harmony or satisfaction. In other words, for those low in anxiety, their perceptions of their relationship harmony and satisfaction did not change in a linear fashion across stage 3 of the separation. However, the relationship perceptions of those higher in attachment anxiety became more negative the longer that they progressed in stage 3. There is some research supporting this notion, that attachment anxiety predicts a decline in marital functioning during major life transitions (Rholes et al., 2001) who found that anxious women's views of spousal support and marital satisfaction continued to worsen (so a negative linear trend) across the transition to parenthood.

In conclusion, consistent with prior research, I found evidence to suggest that attachment dimensions moderate intrapersonal and relationship perceptions during a long-term separation from a spouse. Although prior research has assessed the relevance of attachment dimensions at the actual point of separation (Fraley \& Shaver, 1998), to date no research has assessed the relevance of attachment dimension in predicting emotional or relationship functioning during the ongoing separation particularly in the context of a military deployment. Therefore, extending prior research I found not only that attachment dimensions uniquely predicted perceptions of intrapersonal and relationship functioning, but also that the effects of attachment anxiety were moderated by previous deployment experience and as a function of time separated. Therefore, the effects of attachment anxiety in particular during a separation appear to be both chronic and, for a subset of variables, situational in nature. Specifically, the effects of attachment anxiety on 
relationship perceptions were moderated by (what I believe to be) situations that amplify signals of attachment concerns to a greater extent than were perceptions of emotional well-being and intrapersonal functioning. 


\section{Chapter 3}

\section{Phase One - Reunion Group}

The main purpose of this study was to assess the relationships among attachment anxiety, avoidance, and perceptions of one's interpersonal well-being and relationship quality during the reunion period. I predicted that, in line with the separation findings and with research by Cafferty and colleagues (1994), attachment anxiety in particular would be relevant in predicting how well spouses report that they are coping with the deployment reunion including decreased emotional well-being and relationship perceptions. Further, I predicted that attachment anxiety would be related to perceptions that the reunion period has not lived up to one's original expectations in line with theorizing by Simpson and Rholes (1998). Although Cafferty and colleagues (1994) did not report any significant differences between secure and avoidant athome spouses in their post-reunion functioning, Tidwell, Reis, and Shaver (1996) found that avoidant individuals reported less intimacy, enjoyment and positive emotions with their partners, and that avoidant individuals would structure social activities in ways that minimize intimacy and closeness. Therefore, I also predicted that attachment avoidance could be related to difficulties during the reunion period including decreased emotional well-being and relationship perceptions.

Next, I wanted to investigate whether the relationships between attachment anxiety and post-reunion outcomes are moderated by the number of days they had been reunited with their spouse. I ideally wanted to compare the effects of attachment dimensions at Stage 2 ('Elation') versus stages 3 ('Reintegration': weeks 2-6) and stage 4 ('Renegotiation': weeks 6-12) which are characterized by greater reactivity, increased stress, and decreased coping ability. If individuals high in attachment anxiety are dependent on their partners for emotional comfort, reassurance, 
and validation, experience increased distress in response to partner separations, and desire greater levels of closeness in their relationships, it could be argued that those high in attachment anxiety should respond more positively to stage 2 ('Elation'). However, with more spouses reporting difficulties during stages 3 and 4, it may be the case that these problems may be amplified for those high in attachment anxiety particularly with respect to relationship outcomes (Rholes et al., 2001). In addition, highly avoidant individuals may be less satisfied with their partners' behaviour during stage 2 ('Elation') as they learn to cope with their partners being physically closer, increasing levels of intimacy in the relationship, leading avoidant individuals to feel uncomfortable and potentially increasing conflicts and more unpleasant partner interactions (Tidwell et al., 1996). On the other hand, individuals higher in attachment avoidance might show recovery in stages 3 and 4 from their initial difficulties in stage 2 with the eventual establishment of re-connection and therefore might not report the difficulties that those lower in avoidance might.

For my main analyses, I was interested in testing the hypothesis that individuals with higher levels of attachment anxiety are experiencing greater difficulties with their relationships post-reunion that is mediated by their unrealistically optimistic expectations about what the reunion period might be like. In other words, I was interested in testing whether the relationships between attachment anxiety and difficulties post-reunion are mediated by perceptions that one's original expectations were not met.

Finally, spouses were asked how they felt, and what they did, when they saw their partner for the first time after the deployment. Up until this point, there is little work examining relationships between attachment dimensions and emotions felt during the moment spouses are first reunited with their partners from a long term separation. The literature on mother-child 
interactions indicates that the group of infants that greet their mothers most warmly and are quickly soothed are the secure infants, and that highly anxious infants tend to display emotional ambivalence, oscillating between displays of connection and displays of anger (Ainsworth et al., 1978). Finally, the avoidant infants tend to ignore their mothers more than the other infants, in efforts to suppress their attachment needs. Therefore, it is possible that those who are more 'secure' (lower in anxiety and avoidance) might report the strongest positive emotions during the initial meeting, relative to those higher in attachment anxiety and avoidance.

\section{Method}

\section{Participants and Procedure}

Reunion surveys were sent to 296 spouses/partners of CAF Regular Force personnel (not Augmentees or Reservists, or dual-service couples), who had been deployed, and who had a listed home address as surveys were administered using Canada Post. At the end of the survey, spouses were given the option to be entered into one of five draws for $\$ 100$, as remuneration. The remuneration was provided $100 \%$ by external researchers at Queen's University. A total of 68 spouses completed the survey, a response rate of $23 \%$. The spouses were all women between 22-55 years of age $(M=39.35, S D=8.20)$, and had been married or in a common-law relationship with their partner ${ }^{7}$ for 10.80 years ( $S D=8.76$ years). Out of these 68 spouses, most had partners who were in the $\mathrm{CA}(71 \%, 14 \% \mathrm{RCAF}, 3 \% \mathrm{RCN}, 11 \%$ not disclosed) and had recently returned from a deployment to Afghanistan (89\%). For many of these spouses (65\%), this was not the first time they had been separated from (and reunited with) their partners due to a military deployment, and at the time of completing the survey spouses and partners had been

\footnotetext{
${ }^{7}$ Partners were all men between 24-54 years of age, and were 2 years older than the female spouses on average $(M=$ $41.00, S D=8.35$ ) which is a typical age difference for married samples (e.g., Meltzer, McNulty, Jackson, \& Karney, 2014; Neff \& Geers, 2014).
} 
reunited for an average of 70.95 days $(S D=26.63 \text {, range }=9-181 \text { days })^{8}$. Most of the women indicate that they had children (73\%), most were employed (83\%), and, of those employed, most (79\%) worked outside the home.

\section{Measures}

Attachment Anxiety and Avoidance

Attachment anxiety and avoidance were assessed using the same ECR-R measure from the Separation Survey (see Appendix A). The scale scores for both anxiety (Cronbach's $\alpha=.89$ ) and avoidance (Cronbach's $\alpha=.92$ ) were reliable and were highly correlated ${ }^{9}, r(63)=.70, p<$ .001. Descriptive statistics for both anxiety and avoidance are listed in Table 2.

\section{Relationship Satisfaction}

Relationship satisfaction was assessed using the same RAS measure from the Separation Survey (see Appendix C). With this sample, scale scores for relationship satisfaction had good reliability (Cronbach's $\alpha=.89$ ). Descriptive statistics for this sample are listed in Table 2.

\section{Coping Capacity}

Coping Capacity was assessed using the same 14-item scale from the Separation Survey (see Appendix G). This was a measure developed by the research team so its reliability has not been well established. For this reason, I decided to run a new EFA similar to my previous analyses and in line with recommendations by Fabrigar and Wegener such as using ML as the fitting procedure (2012).

\footnotetext{
${ }^{8}$ It should be noted that number of days reunited was not significantly correlated with attachment anxiety nor avoidance ( $r s=-.17$ and -.10 respectively).

${ }^{9}$ This correlation coefficient is higher than that typically reported in the literature. For example, based on an online sample of over 22,000 individuals, Fraley reports a correlation coefficient between attachment anxiety and attachment avoidance of .41 (Fraley, 2010).
} 
In order to determine the number of factors present amongst these 14 variables, I used the scree test, by examining the scree plot based on eigenvalues from the reduced matrix (see Figure 7). According to this plot, there is a good case for the presence of two factors, with the last final visible drop in the eigenvalues occurring after the second factor (from 1.44 to 0.74 ). However, one could also make a case for the presence of five common factors, with another visible drop in the eigenvalue after factor five (from .48-.23).

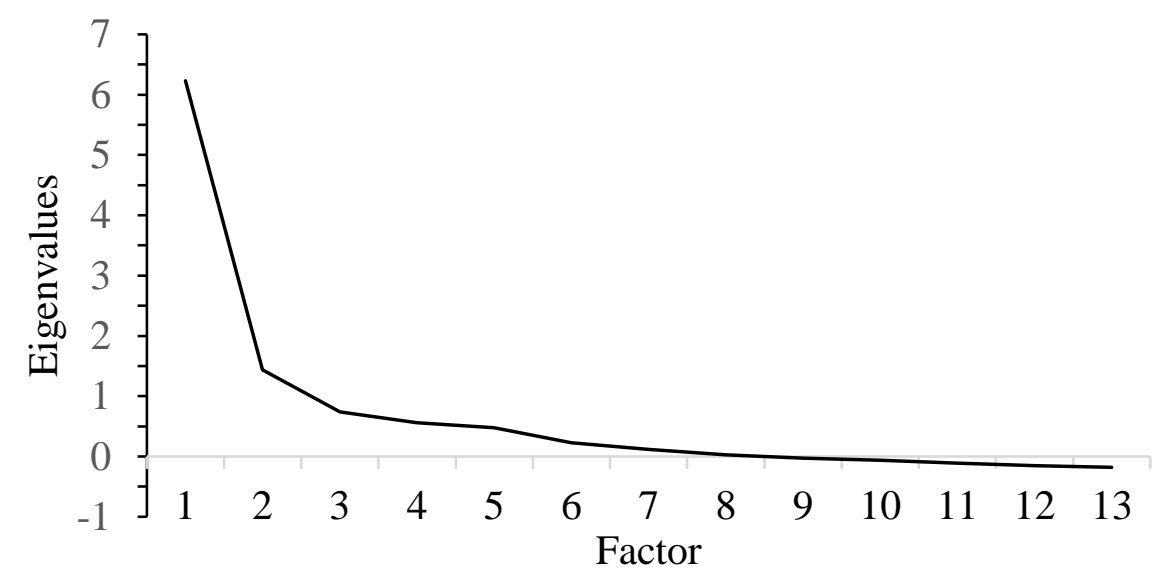

Figure 7. Scree plot of the reduced correlation matrix for coping capacity items (Phase One reunion).

As a secondary test for determining the appropriate number of common factors, I performed a parallel analysis. I used the following parameters: $\mathrm{N}=63$, number of variables $=14$, number of datasets $=100$, and confidence interval $=.95$. According to this analysis, two factors had an eigenvalue greater than those produced by random data, either by the mean or $95^{\text {th }}$ percentile values. Therefore, according to this analysis, the two- factor solution seems appropriate.

The final method I used to determine the appropriate number of factors was assessing model fit by computing the root mean square error estimates for one, two, three, four, and five 
factor models. The one, two, three, and four factor models showed marginal to poor fit (RMSEAs between .17-.09). However, the RMSEA for the five-factor model showed a close fit $(=.04)$. Therefore, while the scree test and parallel analysis indicated that two factors might be appropriate, the model fit index was more consistent with a five-factor model being appropriate. In order to better determine whether a two-factor or five-factor model was appropriate, I next examined the interpretability of the factor loadings. Specifically, I performed EFAs using ML as the extraction method and using an oblique rotation.

To summarize the differences between the two models, the two-factor model included three complex loadings (loadings $>.3$ on more than one factor), and had three low communalities indicating that three items were not strongly related to other items. For two of the complex loadings, the items loaded more strongly onto one factor relative to the other (.98 and $.33 ; .32$ and -.67). On the other hand, the five-factor model contained only one low communality, but five complex loadings and one item that didn't load highly onto any of the five factors. I decided to retain the two-factor solution given the lower number of complex loadings, and better conceptual interpretability relative to the five-factor model.

The communalities for the two-factor model ranged from .26-.87, suggesting that the factors explained a good proportion of the variance in each of the items. Further, the two factors correlated with one another (factor correlation coefficients $=-.44$ ) suggesting that an oblique rotation was appropriate.

The first factor explained $44.5 \%$ of the variance across measures (Eigenvalue $=6.23$ ), was labelled general well-being (11 items: e.g., "I am satisfied with my life"), and reflects feeling energetic, healthy, feeling good about one's marriage, and being able to manage the ups and downs of life. Scores for this factor were calculated by taking the mean of the respective 
items, with higher scores reflecting better physical well-being. The reliability of these scale scores was good (Cronbach's $\alpha=.89$ ).

The second factor explained $10.25 \%$ of the variance amongst items (Eigenvalue $=1.44$ ), was labelled perceived social support (three items: e.g., "I feel supported") and reflects feeling socially supported and less lonely. The scale scores were reliable (Cronbach's $\alpha=.82)$. Although one item would improve alpha by .06 if removed, the alpha was still sufficiently high with this item included so I decided to leave the scale as is, rather than use the item as a separate measure. Descriptive statistics for the coping measures are listed in Table 2.

\section{Reunion Experiences}

I also asked a number of questions about the reunion itself and how the deployment and reunion has potentially affected their perceptions of their partner and the relationship (see Appendix H). For example, spouses were asked whether the reunion met their original expectations $(1=$ not at all, $7=$ very much so $)$, and relative to the time before the deployment whether they now feel more or less emotionally connected and satisfied $(1=$ a lot less connected/satisfied, $7=$ a lot more connected/satisfied) with their partner, and whether things are running more or less smoothly around the house relative to the time that their partner was away ( 1 = much less smoothly, $7=$ much more smoothly). Since the partner's return, I asked spouses to report the quality of their interactions $(1=$ extremely unpleasant, $7=$ extremely pleasant $)$, the extent to which they experience conflict $(1=$ not at all, $7=$ all the time $)$, and when they do experience conflict what issues are most pressing. Finally, I asked spouses in an open-ended fashion to report what they did (in paragraph form) and how they felt (up to three thoughts/emotions) when they saw their partners for the first time. 
Table 2

Descriptive Statistics for Reunion Measures

\begin{tabular}{llcc}
\hline & & $M$ & $S D$ \\
\hline Attachment anxiety & & 2.18 & 0.86 \\
Attachment avoidance & & 2.34 & 1.01 \\
Relationship satisfaction & & 6.36 & 0.57 \\
\hline Coping capacity & General well-being & 5.33 & 0.97 \\
& Perceived social support & 3.50 & 0.71 \\
\hline Reunion experiences & Expectations met & 6.37 & 0.75 \\
& More emotionally connected & 6.33 & 0.74 \\
& More satisfied & 3.75 & 1.59 \\
& Things running more smoothly & 5.31 & 1.54 \\
& Quality of partner interactions & 6.06 & 1.16 \\
& Extent of conflict & 3.16 & 1.72 \\
\hline
\end{tabular}

Note. Response range $=1-7$ (see scale descriptions for specific value labels)

\section{Open-Ended Responses}

For the open-ended responses, I recruited an undergraduate student who was blind to the hypotheses (and all other variables including attachment dimensions) to code these responses. In addition, I recruited another undergraduate student to act as the reliability/validity check and code a portion (30\%) of the main rater's responses. To assess and quantify the emotion/thought list, the student used Shaver, Schwartz, Kirson, and O'Connor's (1987) emotion labelling taxonomy, which was developed after performing a hierarchical cluster analysis on 135 emotion terms. These researchers reported a solution, which organizes emotion labels within an abstractto-concrete hierarchy with six 'basic' emotions comprising the broadest level: love, joy, surprise, anger, sadness, and fear (see Appendix J). Therefore, when using this taxonomy the researcher's goal is to distil all emotion terms to these six basic terms (i.e., identifying which of the six cluster categories the listed emotion belongs to). In total, 63 participants had listed one emotion, 53 had listed two emotions, and 48 had listed three emotions. There was no relationship between 
attachment anxiety or avoidance and the number of emotions listed ( $r$ s equal to .05 and .10 respectively).

Prior to conducting my main analyses, I assessed the validity of the emotion/thought listing coding by assessing the extent of inter-rater reliability. Inter-rater reliability for categorical variables is typically assessed using either percent agreement or Cohen's Kappa (Cohen, 1960; McHugh, 2012). The benefits of using the percent agreement method is that it is easy to calculate and interpret, however it does not take into account the possibility of raters guessing so should be avoided if this possibility is likely. Although Cohen's Kappa takes chance into account, it may result in excessively low estimates given that its assumptions are not well supported when put to the test (see McHugh, 2012). Cohen's Kappa (к) is similar to correlation coefficients in that its values range from -1 to 1 with 0 representing the amount of agreement that would be expected by chance. Cohen recommends the following as a guideline for interpreting к: .01-.20 indicates none to slight agreement, .21-.40 is fair, .41-.60 is moderate, $.61-.80$ is substantial, and .81-1.00 is near perfect agreement. In this case, I used both estimates to assess inter-rater reliability for the first emotion/thought listing as recommended by McHugh. First, I calculated percent agreement by assigning the raters a 1 when their emotion coding matched, and 0 when they were mismatched. Then, I divided the number of ' 1 's assigned by the total number of subjects that listed an emotion/thought. This resulted in near perfect agreement for the first emotion/thought listing (93\% agreement). However, the Cohen's Kappa estimate suggested moderate agreement $(\kappa=.64)$ as it takes into account chance agreement, which in this case was $81 \%$. Specifically, chance agreement for this variable was high given the little variation across emotion listings (with most listing 'joy' related emotions). Overall, these estimates suggest moderate to high agreement and a valid measure of primary emotions described. 


\section{Results}

In order to test whether attachment anxiety, avoidance, and their interaction were associated with difficulties during the reunion period, I conducted multiple linear regressions with attachment anxiety, avoidance, and their interaction term as predictors on the following dependent variables: relationship satisfaction, general well-being, perceived social support, depressive symptoms, expectations met, and quality of partner interactions. Similar to the separation analyses, I tested for the presence of previously reported covariates in the literature on adult attachment and relationship functioning and reunion experiences. Specifically, I performed bivariate correlations between attachment anxiety and relationship length (e.g., Duemmler \& Kobak, 2001; Fraley \& Shaver, 1998) and the presence of children (e.g., Medway, Davis, Cafferty, Chappell, \& O’Hearn, 1995). Neither variable was significantly associated with attachment anxiety ( $r$ s between .12-.14), so I was not concerned about either being potential confounding variables. Following the recommendations of Aiken and West (1991), scores for anxiety and avoidance were mean centered in order to reduce multicollinearity.

Conducting regression analyses with 9 dependent variables, and three predictors can raise concerns regarding inflation of alpha rates. Specifically, by chance I could find 1.35 significant effects. While this is a concern, I also did not have the statistical power to compensate for this type I error inflation without also compromising power and increases in type II error. Specifically, to maintain a .002 alpha rate $(.05 / 27)$, and power at .90 , with three predictors, I

would need a minimum of 120 participants. For this reason, I decided to not implement an alpha correction and maintain the .05 alpha criterion. Therefore, any significant findings should be interpreted with caution. 
There were no significant two-way interactions but there were significant main effects of anxiety and avoidance. Specifically, attachment anxiety was negatively associated with relationship satisfaction $(B=-.25, \beta=-.28, t(59)=-2.24, p=.029)$, general well-being $(B=-.54$, $\beta=-.48, t(59)=-3.12, p=.003)$, perceived social support $(B=-.54, \beta=-.33, t(59)=-2.29, p=$ $.026)$, quality of partner interactions $(B=-.38, \beta=-.30, t(59)=-2.02, p=.048)$, and feeling as though the reunion lived up to expectations $(B=-.64, \beta=-.45, t(59)=-2.85, p=.006)$.

Attachment avoidance was negatively associated with relationship satisfaction postreunion $(B=-.33, \beta=-.44, t(59)=-2.76, p=.001)$.

Next, I wanted to investigate whether the relationships between attachment anxiety and post-reunion outcomes are moderated by the number of days they had been reunited with their spouse. I ideally wanted to compare the effects of attachment dimensions at Stage 2 ('Elation') versus stages 3 ('Reintegration': weeks 2-6) and stage 4 ('Renegotiation': weeks 6-12) which are characterized by greater reactivity, increased stress, and decreased coping ability. Unfortunately, the data from this sample did not allow an adequate test of this hypothesis, because the majority of participants $(n=43)$ completed the survey in stage 3 , with only one participant captured in Stage 2. Another comparison point for the reacting stages 3 and 4 would be stage 5 ('Integration and Acceptance') but again only 10 participants completed the survey in this stage.

What I was able to test, similar to my analyses with the Separation Group, was whether the effects of attachment dimensions are moderated by the number of days reunited. In other words, are the less positive outcomes reported by those high in attachment anxiety (and avoidance) exacerbated closer to or further from the reunion date, relative to those lower in attachment anxiety (and avoidance)? To do this I added the (mean centered) days reunited variable, and the anxiety by days reunited interaction term, to the regression models for outcome 
variables that anxiety significantly predicted (satisfaction, general well-being, perceived social support, depressive symptoms, to what extent expectations were met and quality of interactions). I decomposed any interactions using simple slopes analyses (Aiken \& West, 1991), examining the effects of days reunited at low (-1 SD) and high (+1 SD) attachment anxiety ${ }^{10}$.

First, there was a marginally significant interaction between attachment anxiety and number of days reunited on relationship satisfaction $(B=-.01, \beta=-.19, t(52)=-1.98, p=.053)$. While neither of the slopes reached significance, the simple slope for those high in anxiety was marginal, such that among those high in attachment anxiety, relationship satisfaction decreased across time following reunion (see Figure 8).

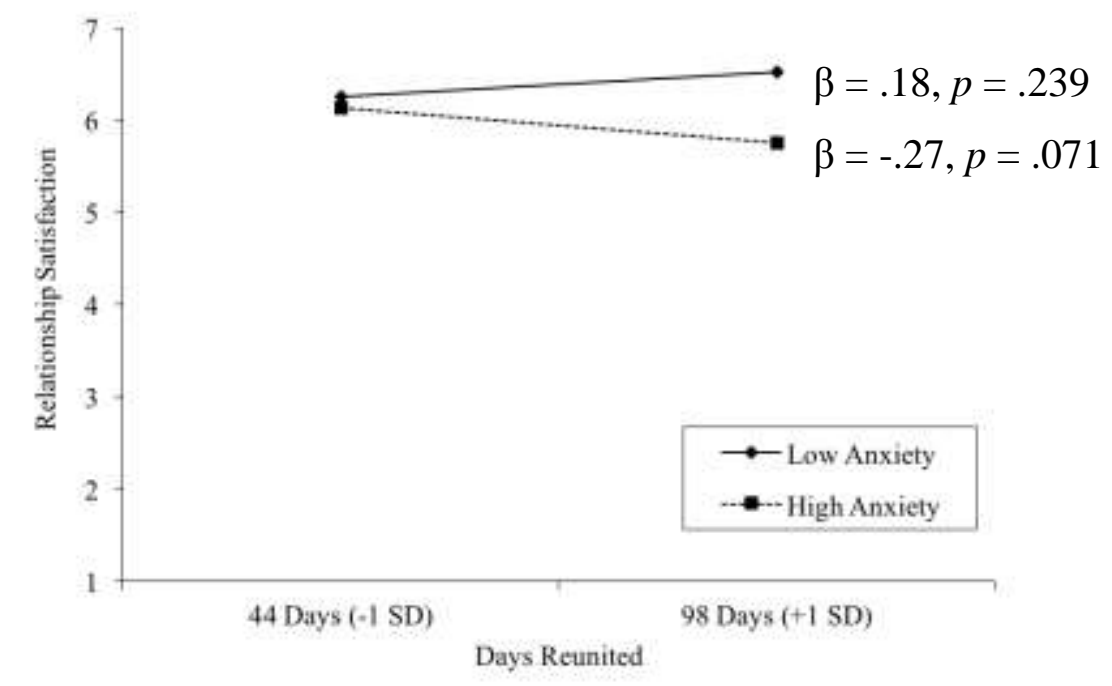

Figure 8. Interaction between attachment anxiety and number of days reunited on relationship satisfaction.

I also found a significant interaction between attachment anxiety and days reunited on quality of partner interactions $(B=-.02, \beta=-.28, t(52)=-2.51, p=.015)$. At low levels of

\footnotetext{
${ }^{10}$ In reality, simple slopes for anxiety were calculated at -1 and +1 SD from the centered mean (so values equal to 26.63 and +26.63 ). The decentered values are presented for better interpretability.
} 
anxiety, there was no relationship between days reunited and quality of interactions $(B=.01, \beta=$ $.14, t(52)=0.85, p=.401$; see Figure 9). However, at high levels of anxiety there was a significant negative relationship between days reunited and quality of interactions $(B=-.02, \beta=$ $-.50, t(52)=-3.04, p=.004)$.

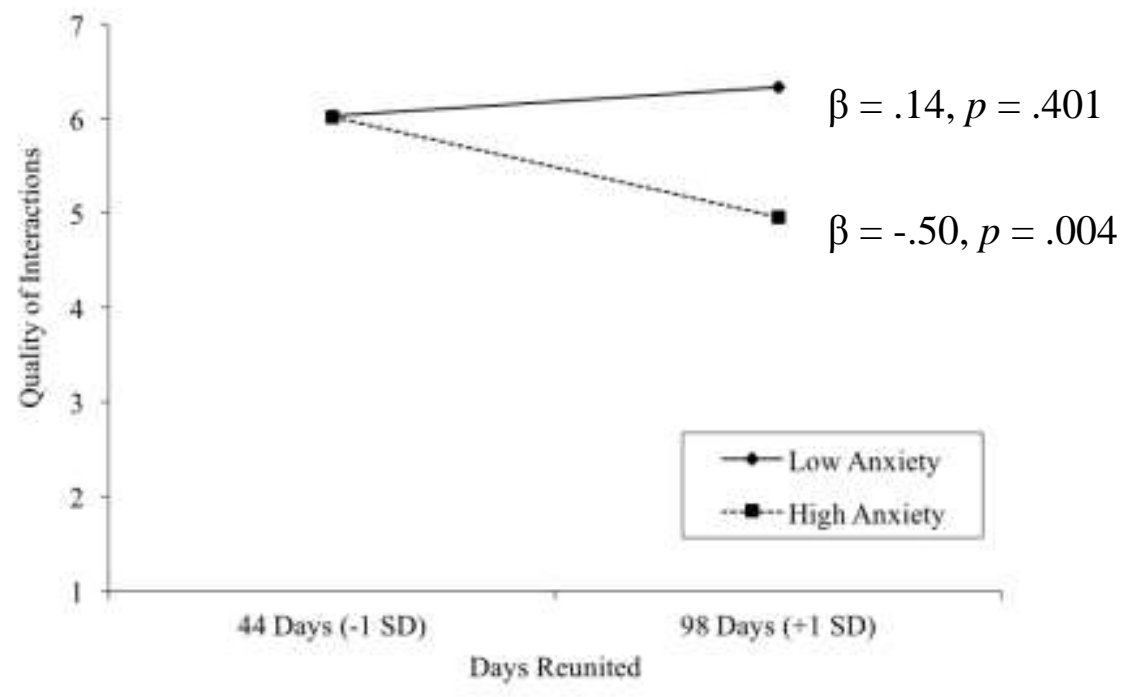

Figure 9. Interaction between attachment anxiety and number of days reunited on quality of partner interactions.

\section{Mediation Models}

For my main analyses, I was interested in testing whether the relationships between attachment anxiety and difficulties post-reunion are mediated by perceptions that one's original expectations were not met. I used the bootstrapping method outlined by Preacher and Hayes (2008) to test a number of mediation models using attachment anxiety as the predictor (controlling for avoidance and the two-way interaction term), and expectations met as the mediator, on all relevant dependent variables that anxiety alone predicted (relationship satisfaction, quality of partner interactions, general well-being, perceived social support, 
depressive symptoms, and satisfaction relative to before the deployment) using the Preacher and Hayes (2008) bootstrapping method. This method is preferred over the widely-adopted Baron and Kenny (1986) mediation method when dealing with small samples. Specifically, the bootstrapping method does not make assumptions about the shape of the distribution of the indirect effect estimate circumventing issues of non-normality and low power (Efron \& Tibshirani, 1993; Mooney \& Duval, 1993; Preacher \& Hayes, 2004; Shrout \& Bolger, 2002). Although there are no specific guidelines with respect to minimum required sample size, Preacher and Hayes state that mathematically the lowest limit would be the sample size minus the total number of predictors plus one. In fact, studies with sample sizes as low as 32 have been published using this method (Cobeanu, 2013). The bootstrapping method (here using 1000 samples) produces an empirically derived confidence interval (CI) of the indirect effect estimate. If zero is not included in the CI, one can conclude that the indirect effect is significant. I performed these analyses using an SPSS multiple mediator macro (available from afhayes.com) that allows for the inclusion of control variables into the tests of indirect effects.

The first model included relationship satisfaction as the criterion. According to this model, the extent that expectations for the reunion were met did mediate the relationship between attachment anxiety and relationship satisfaction (see Figure 10), as zero was not included in the CI (95\% bias-corrected CI: -.22 to -.01). Further, the standardized regression coefficient of the X-Y path (c to c') reduced from -.17 to -.12, and with the $p$ value for the estimate changing from .029 to .146 with the inclusion of expectations met suggesting full mediation $\left(\right.$ model $\left.F(4,58)=17.74, p<.001, R^{2}=.55\right)$. However, it should be noted that the MY path (or b path) was marginal (= .059) meaning that the mediator was not a strong one (in fact some might argue that this model is not valid as a result). For this reason, I remained skeptical 
about drawing inferences about the findings of this model.

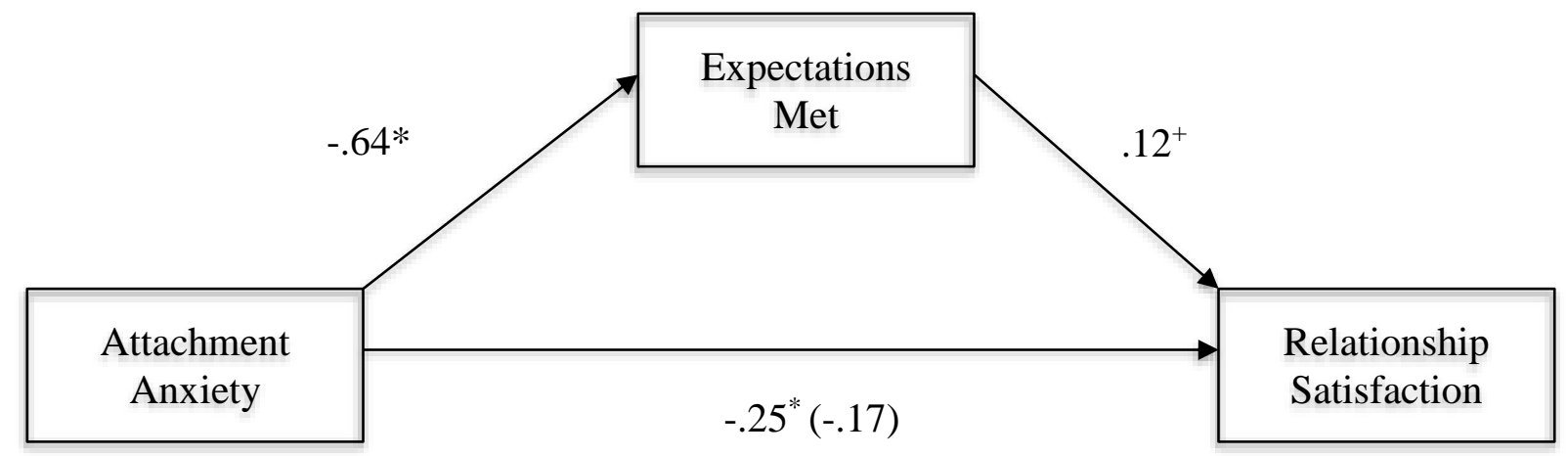

Figure 10. Unstandardized regression coefficients for the relationship between attachment anxiety and relationship satisfaction as mediated by expectations of the reunion being met. The unstandardized regression coefficient between attachment anxiety and relationship satisfaction, controlling for expectations being met, is in parentheses.

${ }^{*} p<.05,{ }^{+} p<.10$.

The second model was tested with quality of interactions as the criterion, and was a stronger model as the mediator more effectively predicted variance in the criterion (see Figure 11). More importantly, zero was not included in the CI (95\% bias-corrected CI: -.65 to -.11). Further, the standardized regression coefficient of the X-Y path (c to c') reduced from -.38 to .03 , and $p$ values increasing from .048 to .803 with inclusion of expectations met suggesting full mediation $\left(\operatorname{model} F(4,58)=21.32, p<.001, R^{2}=.60\right)$.

To better determine the nature of the relationships among attachment anxiety, expectations met, and relationship indices, I also tested alternative models, swapping the order of the dependent measures and mediator. Specifically, I tested models using attachment anxiety as the predictor, extent of expectations met as the dependent measure, and relationship satisfaction and then quality of interactions as the mediators. In both of these models, there were decreases in the X-Y estimates when the mediators were included, however both of the CIs included zero, 
meaning that the mediation models were not significant.

The third, fourth, and fifth models included general well-being, perceived social support, and depressive symptoms as criterions respectively. None of these models came close to demonstrating mediation of any sort (partial or full). The bootstrap results for the indirect effects of attachment anxiety on the three criterions are listed in Table 3. Specifically, for general wellbeing and perceived social support, the extent that expectations were met was not a significant mediator (as demonstrated by the $\mathrm{M} \rightarrow \mathrm{Y}$ path), and for depressive symptoms the $\mathrm{M} \rightarrow \mathrm{Y}$ path was marginal. However, and more importantly, for all three criterions, zero was included in the biascorrected CI suggesting no mediation effects. I also tested the alternative models for these variables, treating the three coping measures as mediators, and extent of expectations met as the dependent measure. None of these models resulted in significant mediation.

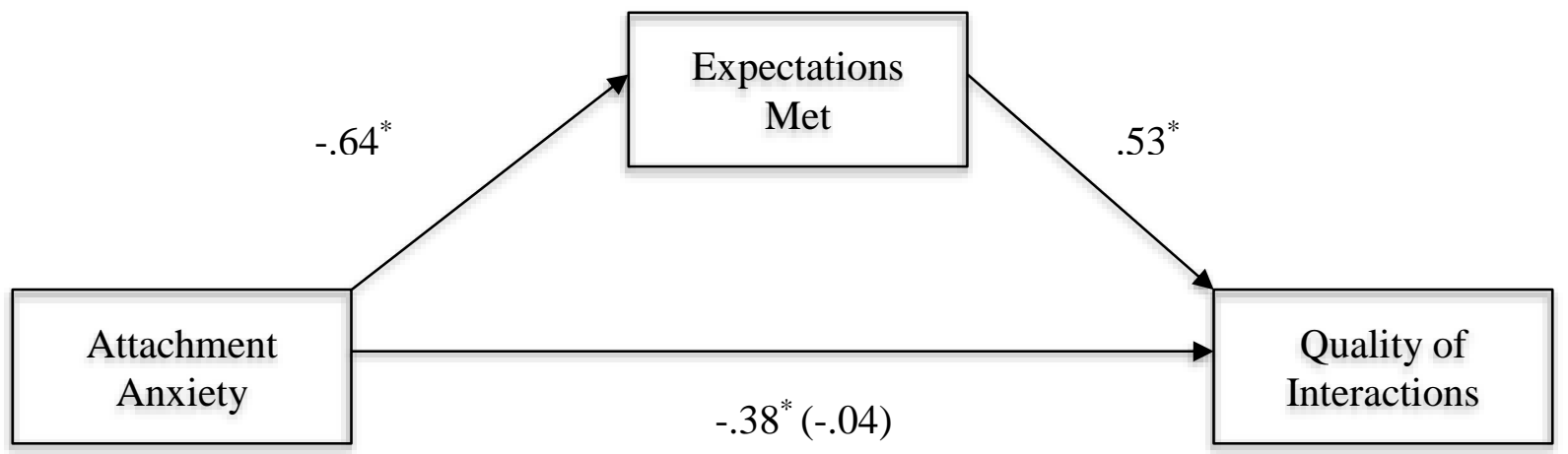

Figure 11. Unstandardized regression coefficients for the relationship between attachment anxiety and quality of partner interactions as mediated by expectations of the reunion being met. The unstandardized regression coefficient between attachment anxiety and quality of interactions, controlling for expectations being met, is in parentheses. ${ }^{*} p<.05,{ }^{+} p<.10$. 
Table 3

Bootstrap Results for the Indirect Effects of Attachment Anxiety on Listed DVs through Expectations Met

\begin{tabular}{|c|c|c|c|c|c|c|}
\hline & & & & & $\begin{array}{l}95 \% \text { Bootstr } \\
\text { bias-correcte }\end{array}$ & $\begin{array}{l}\text { pping } \\
95 \% \mathrm{CI}\end{array}$ \\
\hline Dependent Variable & $\begin{array}{l}\text { Path } a \\
(\mathrm{X} \rightarrow \mathrm{M})\end{array}$ & $\begin{array}{l}\text { Path } b \\
(\mathrm{M} \rightarrow \mathrm{Y})\end{array}$ & $\begin{array}{l}\text { Path } c^{\prime} \\
(\mathrm{X} \rightarrow \mathrm{Y} . \mathrm{M})\end{array}$ & $\begin{array}{l}\text { Path c } \\
(X \rightarrow Y)\end{array}$ & Lower limit & $\begin{array}{l}\text { Upper } \\
\text { limit }\end{array}$ \\
\hline General well-being & $-.64^{*}$ & .05 & $-.46^{*}$ & $-.49^{*}$ & -.20 & .10 \\
\hline Perceived Social support & $-.64^{*}$ & .12 & $-.46^{*}$ & $-.54^{*}$ & -.37 & .10 \\
\hline Depressive symptoms & $-.64^{*}$ & $-.32^{+}$ & & & & \\
\hline $\begin{array}{r}{ }^{*} p<.05 . \\
{ }^{+} p<.10 .\end{array}$ & & & & & & \\
\hline
\end{tabular}

Although attachment anxiety was only marginally predictive of satisfaction with one's partner relative to the time before the deployment, I decided to run the same mediation model with this variable as a criterion to see if the observed findings would be consistent with the first three models which were also relationship-specific outcomes. Similar to the prior models, expectations met mediated the relationship (albeit marginal relationship) between attachment anxiety and satisfaction with one's partner relative to before the deployment (see Figure 12), as zero was not included in the CI (95\% bias-corrected CI: -.67 to -.05). Further, the standardized regression coefficient of the $\mathrm{X}-\mathrm{Y}$ path (c to c') reduced from -.52 to -.27 , and $p$ values increased from .058 to .329 with inclusion of expectations met suggesting full mediation $($ model $F(4,58)=$ $\left.6.31, p<.001, R^{2}=.30\right)$.

I also tested the alternative model, with satisfaction relative to before the deployment as the mediator, and extent of expectations met as the dependent measure. While there was a decrease in the X-Y path (from -.64 to -.50), however the X-Y path remained significant with inclusion of the mediator, implying evidence for only partial mediation. Therefore, the previous model was a stronger one. 


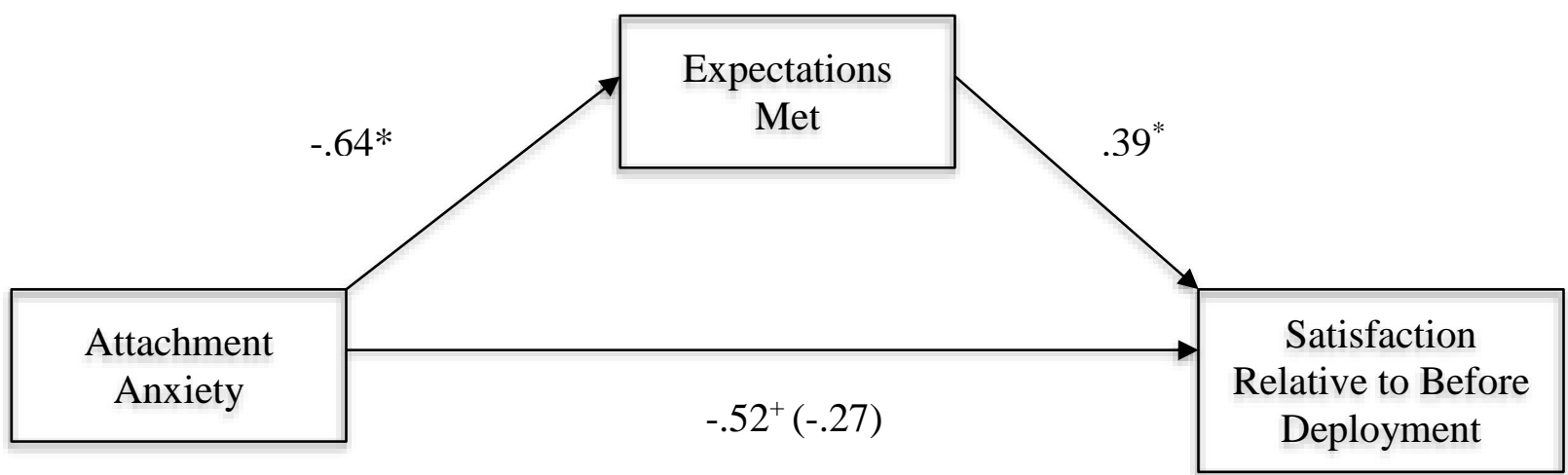

Figure 12. Unstandardized regression coefficients for the relationship between attachment anxiety and satisfaction with one's partner relative to the time before the deployment as mediated by expectations of the reunion being met. The unstandardized regression coefficient between attachment anxiety and satisfaction relative to before deployment, controlling for expectations being met, is in parentheses.

\section{Open-Ended Responses}

Up until this point, there is little work examining relationships between attachment dimensions and emotions felt during the moment spouses are first reunited with their partners from a long term separation. The literature on mother-child interactions indicates that the group of infants that greet their mothers most warmly and are quickly soothed are the secure infants, and that highly anxious infants tend to display emotional ambivalence, oscillating between displays of connection and displays of anger (Ainsworth et al., 1978). Finally, the avoidant infants tend to ignore their mothers more than the other infants, in efforts to suppress their attachment needs. Therefore, it is possible that those who are more 'secure' (lower in anxiety and avoidance) might report the strongest positive emotions, relative to those higher in attachment anxiety and avoidance. In order to assess relationships between attachment anxiety and avoidance, and the nature of emotions listed at the time of the reunion, I performed a discriminant analysis. This analysis assesses whether predictor variables (in this case the 
centered anxiety, avoidance, and interaction terms) can reliably determine group membership, which in this case was the emotion described (out of the six previously outlined). In general, only emotion labels indicating joy, fear, love, and anger were reported (emotion labels related to surprise and sadness were not reported). For the first emotion/thought listing, 83\% of participants reported emotions indicative of joy, 14\% fear, and 3\% love. For the second emotion/thought listing $81 \%$ reported feeling joy, $17 \%$ fear, and $2 \%$ anger. Finally, for the third emotion/thought listing 77\% reported joy, $13 \%$ fear and 10\% love. Given the similar distribution of emotions described in each thought listing and concern over low power for the second and third emotion/thought listings, I will only report results for the assessment of variation in reported emotions as a function of anxiety, avoidance, and their interaction for the first emotion/thought listing which 63 participants completed ${ }^{11}$.

First, the assumption of homogeneity of group variances was upheld, with Box's $M=$ $9.72, F(6,1130)=1.39, p=.217$. Next, none of the tests of equality of group means were significant, meaning that individuals who listed different emotions were not significantly different in terms of levels of anxiety, avoidance, or an interaction between anxiety and avoidance (Wilk's Lambdas ranging from .95-.98, and $p$ values ranging from .23-.56). These initial tests suggest that variance in the predictor variables was not related to the different emotions listed, or the predictor variables were not discriminating emotion labels. Two canonical discriminant functions were produced, one that was largely driven by the prediction of anxiety (with a loading of 1.19), and the other that was driven by the prediction of the anxiety by avoidance interaction term (with a loading of .81). Neither of these functions were associated

\footnotetext{
${ }^{11}$ I did conduct the same analyses on the second and third emotion/thought listings which both resulted in similar, non-significant findings.
} 
with strong canonical correlations (which squared is an estimate of effect size), with canonical coefficients equal to .24 and .17 respectively, which are indices of the strength of the relationships between the predictor variables and the dependent categorical variable. Further, the two functions were not able to predict group membership at a statistically significant level (Wilk's Lambda's $=.92$ and .97 , and $p$ values $=.52$ and .43 respectively). In other words, there was no indication from this analysis that attachment anxiety, avoidance, or their interaction, were associated with different emotions experienced when initially reunited with one's partner.

Participants also described in an open-ended fashion what they did when they saw their partner for the first time. These descriptions were coded by the same primary rater for the degree of physical intimacy described in the initial meeting. Specifically, descriptions were given a rating from 1-4, where 1 represented descriptions with little to no physical contact mentioned, 2 represented some physical intimacy mentioned (hugged or kissed), 3 represented a moderate amount of physical intimacy (a long embrace, kissed and hugged, ran into the partner's arms), and 4 assigned to descriptions that were high in physical intimacy (ran into the partner's arms plus a long embrace or kissing, saying "I love you", crying). This resulted in a variable that was fairly normally distributed, with 7\% assigned a ' 1 ', $23 \%$ assigned a ' 2 ', $49 \%$ assigned a ' 3 ', and $21 \%$ assigned a ' 4 ' for the extent of physical intimacy in the initial meeting.

Similar to prior analyses, I was interested in assessing the extent to which levels of attachment anxiety, avoidance, or an interaction between anxiety and avoidance, are related to reported levels of physical intimacy in seeing one's partner for the first time after the deployment. Fraley and Shaver (1998) found that attachment avoidance was related to decreased physical displays of affection during airport separations. Indeed, attachment avoidance is characterized by discomfort with physical and emotional intimacy. Therefore, I predicted that 
attachment avoidance, more so than attachment anxiety, would be negatively related to lower levels of reported physical intimacy during the initial meeting. To do this, I conducted a multiple linear regression with mean centered anxiety, avoidance, and interaction terms as predictors on the dependent measure physical intimacy during the reunion. Consistent with my predictions, attachment avoidance was a significant predictor of physical intimacy, with avoidance negatively related to intimacy at the reunion, $B=-.32, \beta=-.38, t(53)=-2.04, p=.047$. No other effects approached significance.

\section{Discussion}

Initial research (Cafferty et al., 1994) examining the effects of adult attachment on intrapersonal and relationship outcomes following a military deployment reunion found that secure spouses reported less conflict and more satisfaction relative to insecure spouses, but there were no effects of spousal attachment styles on spousal emotional well-being. The findings from this study suggest that the effects of attachment anxiety, in particular, on outcomes during a reunion period extend beyond relationship perceptions. Specifically, I found that attachment anxiety was related to less effective emotional coping including decreased general well-being, less perceived social support, and more depressive symptoms. In addition, attachment anxiety was related to decreased relationship satisfaction, and feeling more strongly that the reunion has not lived up to one's original expectations. In addition, and extending previous findings, attachment avoidance was also associated with less relationship satisfaction during the postreunion period.

Further extending prior work, and consistent with my separation findings, I found that the effects of attachment anxiety on some relationship outcomes were moderated by time reunited. For example, for highly anxious individuals their perceptions of their interactions with 
their partners declined as a function of time reunited. However, there was no relationship between quality of interactions and time reunited for those lower in attachment anxiety. This is consistent with the findings of Rholes and colleagues (2001) who found that for anxious spouses, their perceptions of their partner's support declined across the transition into parenthood.

In addition, I was able to identify one potential mechanism to explain why higher levels of attachment anxiety are related to more negative relationship perceptions during the reunion period - beliefs that one's original expectations have not been met. Indeed, we found that the relationships between attachment anxiety and decreased relationship satisfaction and quality of partner interactions were fully mediated by beliefs that the reunion period has not lived up to one's original expectations. In explaining the findings by Rholes and colleagues (2001), Simpson and Rholes (2008) speculate that the negative outcomes experienced by high anxiety individuals during the transition to parenthood might be tied to their unrealistically optimistic views of their romantic partners and relationships. Specifically, anxious individuals may become frustrated and dissatisfied because they perceive that their partners are not living up to their expectations.

Finally, consistent with the airport findings of Fraley and Shaver (1998), higher levels of attachment avoidance were related to reports of less physical intimacy during the initial meeting of the reunion relative to lower levels of avoidance. Increases in intimacy tend to make avoidant individuals uncomfortable, so they often engage in distancing behaviours towards their partners or structure activities such as to reduce intimacy and closeness (Tidwell et al., 1996). Again, this is more evidence that highly avoidant individuals avoid intimacy-building opportunities within their relationships, relative to those lower in avoidance. 
Unfortunately, I was not able to test the relevance of attachment dimensions in predicting intrapersonal adjustment and relationship perceptions according to identified reunion stages (Egerton-Graham, 2009), some of which typically entail greater stress and emotional reactivity than others. This was due to a lack of variance, or restricted range, in time reunited when the spouses were surveyed. Too few spouses were surveyed outside of the stage 3 window, either in the stages preceding or following that stage to allow for adequate testing as a function of stage progression. Fortunately, however, these are questions that can be addressed with the larger and more adequately powered large-scale survey described later.

Finally, some might question whether the effects of attachment dimensions have more specific parameters, centered around the transition periods, and that the effects of attachment dimensions become more or less irrelevant as time goes on. While some analyses as a function of time separated and time reunited point to the continued importance of attachment anxiety, in particular, in explaining spousal adjustment across time, the purpose of the next chapter was to continue to assess the overall importance of attachment anxiety and attachment avoidance in a longitudinal design that tracked the same group of participants over 4 months of an overseas deployment. Most importantly, different from the other phases, this study assessed how frequently and intimately partners communicated with one another from afar, spouse's perceptions of the quality of emotional support received by one's partner, and provided to one's partner, how provision of emotional support is distributed within the relationship, and also the extent to which spouses fantasize about their partners and their return. 


\section{Chapter 4}

\section{Phase Two - Longitudinal Study}

Consistent with findings from Fraley and Shaver (1998) and Cafferty and colleagues (1994), as well as the findings from Phase One, I predicted that individuals higher in attachment anxiety would report greater difficulties during the separation relative to individuals lower in attachment anxiety given their strong desires for closeness and intimacy and their reliance on attachment figures for emotional regulation and support. Further, I predicted that attachment anxiety will be related to more negative relationship perceptions (e.g., Collins \& Read, 1990). Specifically, I predicted that higher levels of attachment anxiety would be associated with decreased coping capacity, and lower quality partner interactions, decreased relationship satisfaction, and some difficulties providing emotional support to one's partner (Collins \& Feeney, 2000) during the ongoing separation. In addition, I predict levels of attachment anxiety to be related to greater fantasizing about the reunion, given that highly anxious individuals are more dependent on their partners for validation and support in general, regardless of the context (e.g., Davilla, 2001; Wei, Mallinckrodt, Larson, \& Zakalik, 2005).

On the other hand, I predicted that attachment avoidance would be associated with deactivating strategies in response to environmental threats such that attachment avoidance would not be associated with decreased coping capacity, but would be related to more negative partner perceptions, lower quality partner interactions, decreased relationship satisfaction, and more discomfort in seeking emotional support from one's partner (Collins \& Read, 1990). Further, individuals higher in attachment avoidance might be less likely to fantasize about their partner's return given their ability and motivation to decrease the activation of thoughts related to abandonment (Fraley \& Shaver, 1997, Study 1). 
Next, I was interested in assessing the relationships among attachment dimensions and post-reunion outcomes similar to Phase One. Consistent with previous research (Cafferty et al., 1994; Rholes et al., 2001) and the findings from Phase One, I predicted that attachment anxiety would be related to less effective emotional coping including self-blaming (Mayseless et al., 1996), and more negative perceptions of relationship functioning including decreased relationship satisfaction, and feeling more strongly that the reunion has not lived up to one's original expectations. Further, in line with my findings from Phase One, I predicted that attachment avoidance would be associated with less relationship satisfaction during the postreunion period.

Finally, I was interested in testing mediation models to assess relationships between attachment orientations and perceptions of relationship and intrapersonal functioning during the ongoing separation (e.g. perceptions of emotional support provision and acceptance).

\section{Method}

\section{Participants and Procedure}

After completing their Separation Survey, participants were invited to participate in a longitudinal study that entailed completing monthly surveys (up to a total of four) and an additional reunion survey (identical to that used in Phase One) once their partners had returned home. They were informed that for completion of each survey, and with their consent, their names would be entered into one of two draws for $\$ 500$ as remuneration. The remuneration was provided $100 \%$ by external researchers at Queen's University. Out of the 85 participants that participated in the initial Separation Survey, $62(71 \%)$ agreed to participate in this longitudinal 
component $^{12}$. Out of the 62 who agreed to participate, $50(81 \%)$ completed at least one monthly survey, $31(50 \%)$ completed at least 3 monthly surveys, and $36(66 \%)$ completed the final reunion survey.

\section{Monthly Survey Measures}

\section{Coping Capacity}

I retained a total of 12 items from the Phase One survey coping measure, and added eight more items to more adequately assess perceptions of one's physical well-being (e.g., "I feel nauseous most of the time."). In addition, I also removed two items measuring perceptions of one's marriage (which I felt were already measured by relationship indices) and satisfaction with life. Therefore, a total of 20 items were created by the researchers (see Appendix I) that assess coping capacity or general well-being with facets such as emotional (e.g., "I feel content most of the time."), physical (e.g., "I feel nauseous most of the time”), social (e.g., "I feel good about my social life"), and practical (e.g., "I wish I had more help around the house") functioning. Respondents indicated their agreement with these items on a scale from 1 (Strongly Disagree) to 7 (Strongly Agree). In order to assess the factor structure of these items (given the lack of theoretical framework for the development of this scale), I decided to conduct an EFA on the first month's coping items and then calculate reliability estimates confirming the psychometric properties of such latent variables for other months' coping items. Although this is not the ideal way to factor analyze multilevel data, multilevel factor analyses typically require a minimum of 50 groups (specifically between 50-100; see Muthen, 1994), of which I only had four (four months).

\footnotetext{
12 There were no differences in levels of anxiety, avoidance, or relationship satisfaction between those who did and did not participate in Phase 2
} 
In line with recommendations by Fabrigar and Wegener (2012), I first wanted to determine the appropriate number of common factors that could best explain intercorrelations among items (ranging between .01-.78). Specifically, I assessed the scree plot based on eigenvalues from the reduced matrix (see Figure 13). According to this plot, there is a good case for the presence of one factor, with the only visible and sharp drop in variance explained, evidenced by eigenvalues, occurs after the first factor (from 8.10 to 1.68 ).

As a secondary test for determining the appropriate number of common factors, I performed a parallel analysis. I used the following parameters: $\mathrm{N}=50$, number of variables $=20$, number of datasets $=100$, and confidence interval $=.95$. According to this analysis, three factors had eigenvalues greater than those produced by random data, either by the mean or $95^{\text {th }}$ percentile values. Therefore, according to this analysis, a three- factor solution seems appropriate.

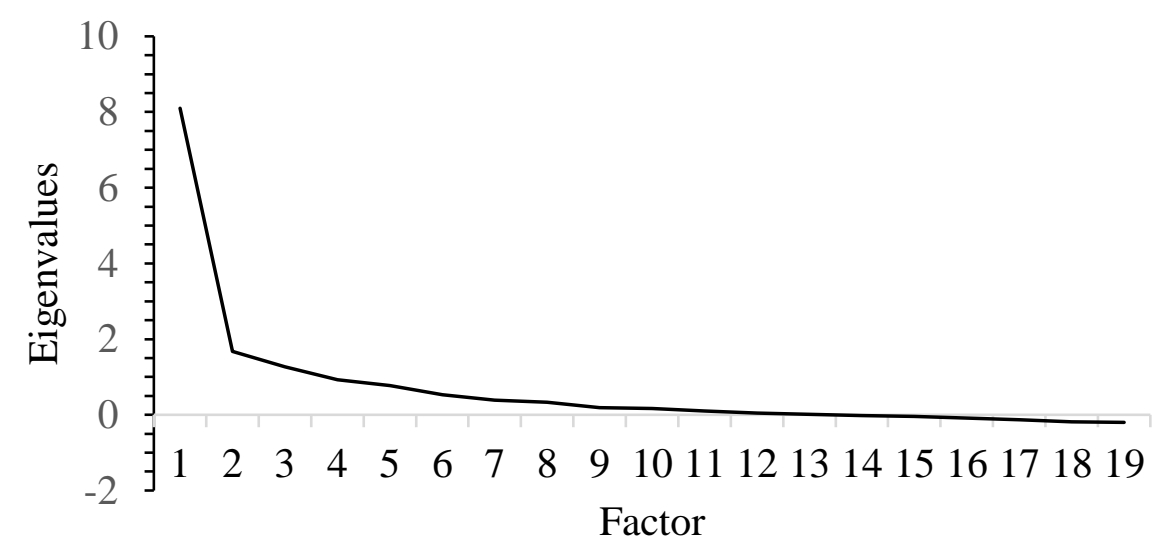

Figure 13. Scree plot of the reduced correlation matrix for coping capacity items (Phase Two first month).

The final method I used to determine the appropriate number of factors was assessing model fit using the root mean square error estimates for one, two, and three factor models. The one and two factor models showed poor and a marginal fit, respectively (RMSEAs $=.114$ and 
.097). However, the RMSEA for the three-factor model showed an acceptable fit (=.064).

Therefore, while the screetest indicated a clear drop in variance explained after the first factor, the parallel analysis and RMSEA fit indices indicated that three factors might be more appropriate. In order to better determine whether a one-factor or three-factor model was more appropriate, I examined the interpretability of the factor loadings. Specifically, I performed EFAs using ML as the extraction method and using an oblique rotation.

To summarize the differences between the two models, the one-factor model contained six items with low communalities, however all items loaded highly $(>.30)$ onto the factor except one item. The three-factor model had only three items with low communalities, however there were seven items with complex loadings (loading $>.30$ on more than 1 factor). The factors were correlated with one another (factor correlation coefficients from .20-.47) suggesting an oblique rotation was appropriate. Given the large number of complex loadings, I decided to retain the one-factor solution. This factor explained $40.49 \%$ of variance among items (Eigenvalue $=8.10$ ). Further, the reliability of scale scores was excellent (Cronbach's $\alpha=.91$ ) according to George and Mallery (2003), and no items would improve this coefficient if removed (including the low loading item "I make an effort to eat well).

I then calculated the Cronbach's $\alpha$ coefficients for the three other months assuming a one-factor structure was appropriate. The reliability estimates for months two, three, and four were all strong with Cronbach's $\alpha$ ranging between .93-.94. Therefore, the one factor solution was appropriate for the coping scale across all months. Overall coping capacity factors were calculated by taking the mean of the respective 20 items (with some reverse scored as required). See Table 4 for descriptive statistics.

\section{Relationship Satisfaction}


I used the RAS measure (Hendrick, 1988; see Appendix C) from the Phase One surveys. This scale has six items (e.g., "How much do you love your partner?”) and responses are indicated on a scale from 1 (Strongly Disagree) to 7 (Strongly Agree). Higher scores indicate greater relationship satisfaction. Given this was an established scale, I simply calculated the internal consistency of the scale for each month. The reliability across all months was good, with Cronbach's $\alpha$ coefficients for scale scores between .83 to .89 . Total scores for relationship satisfaction were then calculated using the mean of all 6 items (after reverse coding negatively phrased items). Descriptive statistics can be found in Table 4.

\section{Emotional Support}

Spouses were asked to complete five items that tapped into perceptions of emotional support in the relationship developed by the research team. Specifically, participants were asked to report how the emotional support provision is divided in the relationship (using percentages totalling 100), whether they are satisfied with the emotional support they receive, how comfortable they are providing emotional support to their partners, whether they tend to "push" their partners away when they provide emotional support, and whether they are comfortable expressing vulnerability to their partners. Respondents indicated their agreement with these last four items using a Likert-type scale from 1 (strongly disagree) to 7 (strongly agree).

The factor structure of the latter four items (similar scale) was not known, so I conducted analyses similar to those reported for the scales above, in line with recommendations by Fabrigar and Wegener (2012), to assess the psychometric properties of this scale. To determine the appropriate number of common factors, I first examined the scree plot based on eigenvalues from the reduced matrix (see Figure 14). According to this plot, there is a good case for the 
presence of one factor, with the only visible and sharp drop in variance explained, evidenced by eigenvalues, occurs after the first factor (from 1.22 to .18).

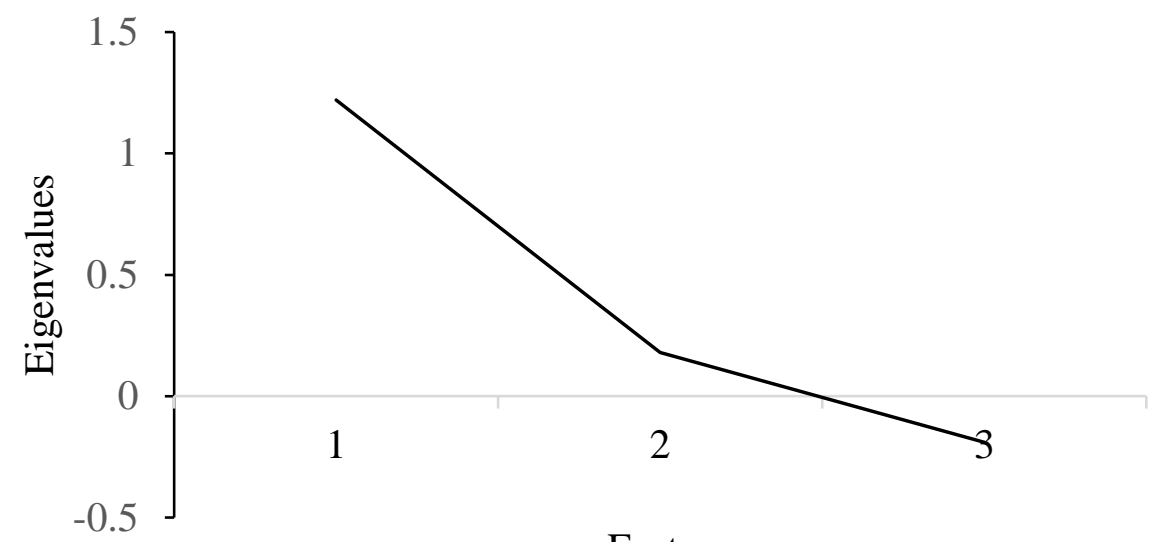

Factor

Figure 14. Scree plot of the reduced correlation matrix for emotional support items (Phase Two first month).

As a secondary test for determining the appropriate number of common factors, I performed a parallel analysis. I used the following parameters: $\mathrm{N}=50$, number of variables $=4$, number of datasets $=100$, and confidence interval $=.95$. According to this analysis, two factors had eigenvalues greater than those produced by random data, either by the mean or $95^{\text {th }}$ percentile values. Therefore, according to this analysis, a two-factor solution seems appropriate.

The final method I used to determine the appropriate number of factors was computing the root mean square error estimates for one, two, and three factor models. The one factor model showed a poor fit (RMSEAs $=.14$ ), and the two-factor model (and beyond) indices could not be computed due to too few degrees of freedom. Therefore, although the scree test suggested a onefactor model might be most appropriate, the RMSEA indicated poor fit, and the parallel analysis pointed to a two-factor model. In order to better determine whether a one-factor or two-factor 
model was more appropriate, I examined the interpretability of the factor loadings. Specifically, I performed EFAs using ML as the extraction method and using an oblique rotation.

Unfortunately, and due to low power, the two-factor model could not be successfully run. Regarding the one-factor model, two of the items had low communalities $(<.30)$ suggesting that some variables were not strongly related to other variables in the scale, although all variables had decent factor loadings $(>.30)$ on the single factor (loadings from .46-.73). However, when I calculated the Cronbach's $\alpha$ reliability estimate, the scale scores demonstrated questionable internal consistency $(\alpha=.62)$ according to George and Mallery (2003). When I calculated the reliability estimates for this four-item scale for the three other months, all estimates were in the poor to questionable range (between .52-.62). I also examined bivariate correlations among all items aggregating across all months, and the inter-item correlations ranged from .12-.44 suggesting only weak to moderate correlations. For this reason, I decided to treat all items as separate measures for this scale (see Table 4 for descriptives).

\section{Expectations for the Reunion}

A total of four items tapped into expectations for the partner's return. Spouses were asked the extent to which they think about their partner often, fantasize about their partner and their return, whether they believe life would improve once their partner is home, and how eager they are for their return on a scale from 1 (strongly disagree) to 7 (strongly agree). Consistent with my analyses for previous scales, I conducted EFAs in line with recommendations by Fabrigar and Wegener (2012), to assess the psychometric properties of this scale. To determine the appropriate number of common factors, I first examined the scree plot based on eigenvalues from the reduced matrix (see Figure 15). According to this plot, there is a good case for the 
presence of one factor, with the only visible and sharp drop in variance explained, evidenced by eigenvalues, occurs after the first factor (from 1.44 to .11).

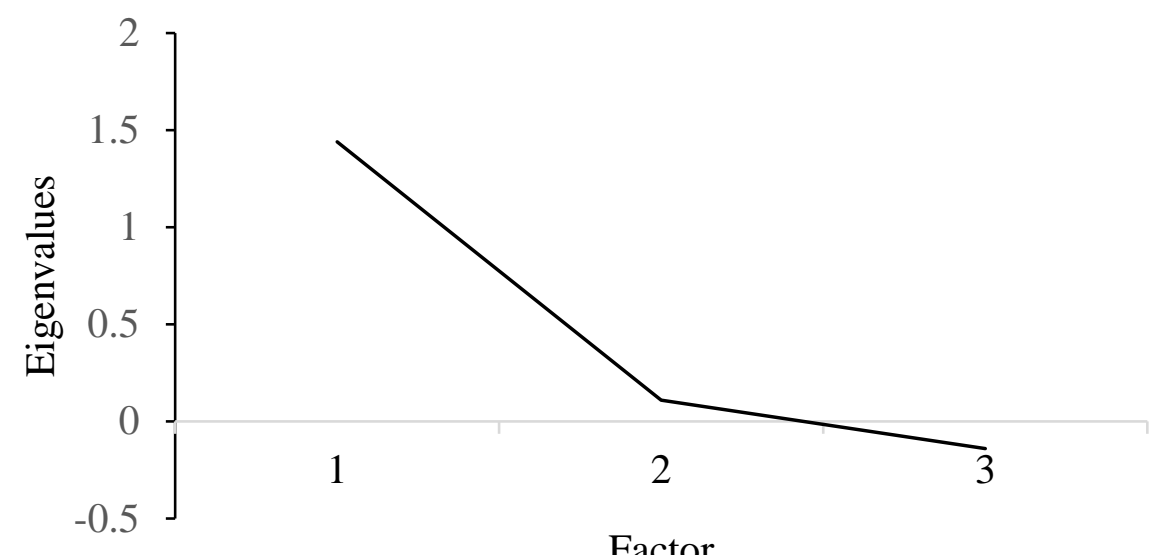

Figure 15. Scree plot of the reduced correlation matrix for expectations for the reunion items (Phase Two first month).

As a secondary test for determining the appropriate number of common factors, I performed a parallel analysis. I used the following parameters: $\mathrm{N}=50$, number of variables $=4$, number of datasets $=100$, and confidence interval $=.95$. According to this analysis, only one factor had an eigenvalue greater than those produced by random data, either by the mean or $95^{\text {th }}$ percentile values. Therefore, according to this analysis, a one-factor solution seems appropriate.

The final method I used to determine the appropriate number of factors was by computing the root mean square error estimates for the one-factor model. The one factor model showed a marginal fit $(\mathrm{RMSEA}=.10 . \mathrm{I}$ then examined the interpretability of the factor loadings, by performing an EFA using ML as the extraction method and using an oblique rotation. The factor explained $39 \%$ of variance among variables (Eigenvalue $=1.56$ ), but one item had a low communality (= .164). All factor loadings were above .30 (from .41-.78). However, this scale's scores demonstrated poor internal consistency (Cronbach's $\alpha=.57$ ). These findings were 
consistent across all months' responses, with internal consistency estimates between .58-.69 which are in the poor to questionable range (George \& Mallery, 2003). Further, inter-item correlations aggregated across all months ranged from .32-.54 suggesting moderate correlations between items. These findings indicate that items are distinct enough that they might be best treated as separate measures. For this reason, I treated these items as separate measures (see Table 4 for descriptive statistics).

\section{Communication and Quality of Interactions}

Spouses were asked questions about their communication with their partners.

Specifically, spouses were asked how often they communicated with their partners in an openended fashion. Responses ranged from multiple times daily, to once a week. Therefore, these responses were coded to represent the number of times they communicated with their partners per week. Next, spouses were asked how they communicated with their partners. Methods of contact were rank ordered from least intimate $(1=$ email $)$ to most intimate $(5=$ in person $)$. Finally, spouses were asked to provide details about the last interaction they had with their partners. For example, spouses were asked about the quality of that interaction from 1 (very unpleasant) to 7 (very pleasant), and whether they felt worse or better immediately after the interaction and the following day from 1 (much worse) to 7 (much better). Descriptive statistics for all measures are listed in Table 4. 
Table 4

Descriptive Statistics for Monthly Survey Measures

\begin{tabular}{|c|c|c|c|c|c|c|c|c|}
\hline \multirow[b]{2}{*}{ Measure } & \multicolumn{2}{|c|}{ Month 1} & \multicolumn{2}{|c|}{ Month 2} & \multicolumn{2}{|c|}{ Month 3} & \multicolumn{2}{|c|}{ Month 4} \\
\hline & $M$ & $S D$ & $M$ & $S D$ & $M$ & $S D$ & $M$ & $S D$ \\
\hline Coping capacity ${ }^{1}$ & 5.00 & 1.02 & 5.10 & 1.11 & 4.95 & 1.19 & 4.91 & 1.16 \\
\hline Relationship satisfaction ${ }^{1}$ & 6.26 & 0.81 & 6.42 & 0.57 & 6.53 & 0.46 & 6.43 & 0.63 \\
\hline $\begin{array}{l}\text { Satisfaction with emotional } \\
\text { support received }{ }^{1}\end{array}$ & 5.92 & 1.22 & 6.10 & 1.01 & 6.28 & 1.00 & 6.06 & 1.22 \\
\hline Push away partner ${ }^{1}$ & 2.39 & 1.50 & 2.57 & 1.66 & 2.36 & 1.51 & 2.33 & 1.63 \\
\hline Like providing support ${ }^{1}$ & 6.35 & 0.86 & 6.55 & 0.59 & 6.44 & 0.68 & 6.48 & 0.67 \\
\hline $\begin{array}{l}\text { Comfort expressing } \\
\text { vulnerability }{ }^{1}\end{array}$ & 5.53 & 1.66 & 5.86 & 1.37 & 5.69 & 1.44 & 5.91 & 1.18 \\
\hline $\begin{array}{l}\text { Provision of emotional support } \\
(\% \text { self })^{2}\end{array}$ & 57.43 & 14.77 & 52.02 & 8.48 & 50.64 & 10.21 & 55.45 & 11.41 \\
\hline Think about partner often & 6.78 & 0.47 & 6.76 & 0.48 & 6.69 & 0.52 & 6.82 & 0.39 \\
\hline Eager for partner's return & 6.78 & 0.47 & 6.71 & 0.64 & 6.85 & 0.37 & 6.88 & 0.33 \\
\hline $\begin{array}{l}\text { Fantasize about partner's } \\
\text { return }{ }^{1}\end{array}$ & 5.92 & 1.34 & 5.90 & 1.48 & 5.95 & 1.41 & 5.94 & 1.37 \\
\hline Life will improve once home ${ }^{1}$ & 6.12 & 1.03 & 6.05 & 1.23 & 6.13 & 1.15 & 6.06 & 1.35 \\
\hline $\begin{array}{l}\text { Frequency of communication } \\
\text { (times/week) }\end{array}$ & 6.90 & 4.80 & 6.55 & 2.58 & 6.74 & 3.68 & 7.85 & 5.11 \\
\hline Method of communication ${ }^{4}$ & 3.06 & 1.13 & 3.00 & 1.27 & 2.79 & 1.15 & 2.73 & 1.15 \\
\hline Quality of last interaction ${ }^{3}$ & 6.33 & 1.09 & 6.36 & 1.12 & 6.15 & 1.11 & 6.42 & 0.87 \\
\hline $\begin{array}{l}\text { How did you feel immediately } \\
\text { after }\end{array}$ & 5.73 & 1.15 & 5.79 & 1.20 & 5.33 & 1.24 & 5.67 & 1.32 \\
\hline How did you feel the next day & 4.67 & 0.89 & 4.82 & 0.98 & 5.08 & 1.17 & 5.00 & 1.20 \\
\hline $\begin{array}{l}{ }^{1} \text { Response range }=1-7 \text { (see scal } \\
\text { labels) } \\
{ }^{2} \text { Response range }=1-100 \\
{ }^{3} \text { Response range }=1-21 \\
{ }^{4} \text { Response range }=1-4\end{array}$ & escri & & & & & & & \\
\hline
\end{tabular}

\section{Reunion Survey Measures}

The following are the same measures from the reunion survey distributed in Phase One. 
Attachment anxiety and avoidance were assessed using the same ECR-R measure from Phase One (see Appendix A). The scale scores for both anxiety (Cronbach's $\alpha=.93$ ) and avoidance (Cronbach's $\alpha=.89$ ) were reliable and were highly correlated, $r(36)=.73, p<.001$. Descriptive statistics for both anxiety and avoidance are listed in Table 5. Relationship Satisfaction

Relationship satisfaction was assessed using the RAS (Hendrick, 1988; see Appendix C). With this sample, relationship satisfaction was a reliable measure (Cronbach's $\alpha=.88$ ). Descriptive statistics for this sample are listed in Table 5.

\section{Coping Capacity}

Coping Capacity was assessed using the same 14-item scale from the Phase One surveys (see Appendix G). This measure produced different factor structures for both the separation and reunion samples, so a clear framework of latent variables was not yet well established. For this reason, I decided to run EFA analyses similar to prior analyses.

To determine the appropriate number of common factors, I first examined the scree plot based on eigenvalues from the reduced matrix (see Figure 16). According to this plot, there is a good case for the presence of either three or four factors, with visible drops in eigenvalues occurring both after the third (from 1.17 to .73) and fourth (from .73 to .38 ) factors.

As a secondary test for determining the appropriate number of common factors, I performed a parallel analysis. I used the following parameters: $\mathrm{N}=41$, number of variables $=14$, number of datasets $=100$, and confidence interval $=.95$. According to this analysis, only one factor had an eigenvalue greater than those produced by random data, either by the mean or $95^{\text {th }}$ percentile values. Therefore, according to this analysis, a one-factor solution seems appropriate. 


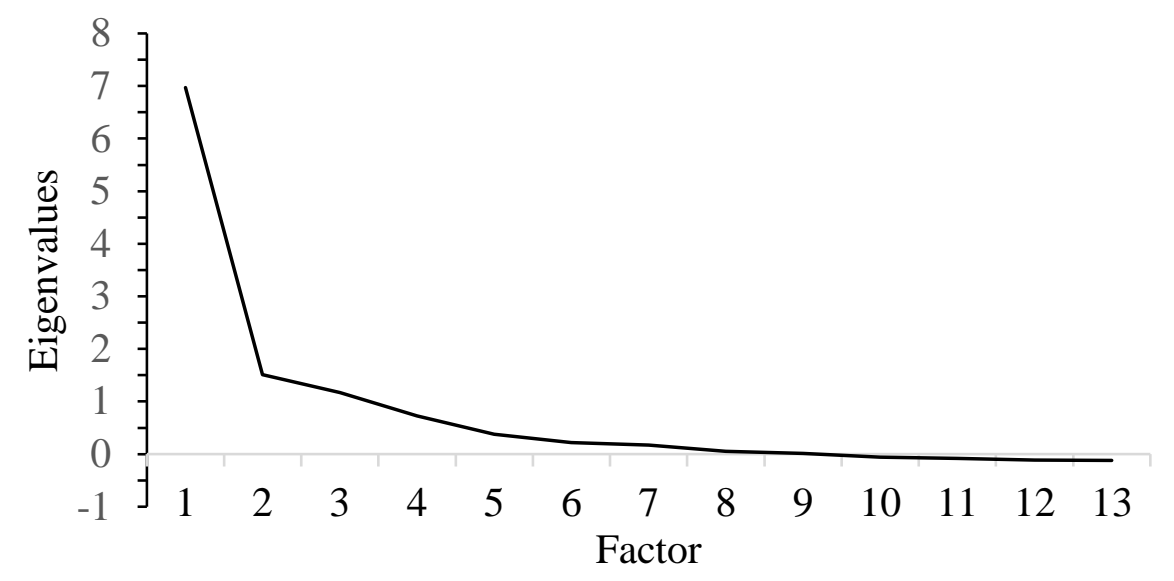

Figure 16. Scree plot of the reduced correlation matrix for coping capacity items (Phase Two reunion).

The final method I used to determine the appropriate number of factors was assessing model fit using the root mean square error estimates for the one, three, and four-factor models. The one-factor model (RMSEA = .18) and the three-factor model (RMSEA =.12) showed poor fit, and the four-factor model showed an acceptable fit (RMSEA $=.07)$. Therefore, while the scree plot shows that either a three or four-factor model might be appropriate, the parallel analysis indicated a one-factor solution, and the goodness of fit index pointed to the appropriateness of a four-factor solution. In order to better determine how many common factors would be appropriate, I examined the interpretability of the factor loadings for the three and four-factor models, by performing an EFA using ML as the extraction method and using an oblique rotation.

To summarize the findings from both models, the four-factor model had no variables with low communalities and one complex loading. However, interpreting the factors conceptually was more challenging, partly because two of the factors were comprised of only two variables. On the other hand, the three-factor model also contained only one complex 
loading, contained one variable with a low communality, but was more parsimonious and conceptually more interpretable. Further, all factors contained at least three variables making it easier to also calculate other estimates of internal consistency. For this reason, I decided to retain the three-factor model and report the findings as follows.

Factor one explained $49.81 \%$ of variance in measures (Eigenvalue $=6.97$ ), was labelled well-rested (four items: e.g., "I have been sleeping well”), and reflects feeling energetic, well rested, and the ability to manage daily tasks. Scores for this factor were calculated by taking the mean of the respective items, with higher scores reflecting feeling more well-rested. This scale was found to have good reliability (Cronbach's $\alpha=.84$ ), and no items would improve this value if removed.

Factor two explained $10.76 \%$ of variance in measures $($ Eigenvalue $=1.51)$, was labelled general well-being (seven items: e.g., "I am satisfied with my life"), and reflects feeling satisfied with one's self, financial situation, and social life. Scores for this factor were calculated by taking the mean of the respective items, with higher scores reflecting feeling more well-rested. This scale was found to have excellent reliability (Cronbach's $\alpha=.91$ ), and no items would improve this value if removed.

Finally, factor three explained $8.34 \%$ of variance in measures (Eigenvalue $=1.17$ ), was labelled perceived relationship support (three items: e.g., "I am satisfied with my marriage"), and reflects feeling satisfied with one's marriage, feeling supported, and not lonely. Scores for this factor were calculated by taking the mean of the respective items, with higher scores reflecting feeling more well-rested. This scale was found to have good reliability (Cronbach's $\alpha$ $=.83$ ), and no items would improve this estimate if removed. Descriptive statistics for these measures are provided in Table 5. 


\section{Reunion Experiences}

Items from this section of the survey were identical to those listed in the reunion survey from Phase One. Descriptives for the scaled items are provided in Table 5. Aside from the scale items, the open-ended response items were also included in this survey. Specifically, spouses were asked to report what they did (in paragraph form) and how they felt (up to 3 thoughts/emotions) when they saw their partners for the first time.

Table 5

Descriptive Statistics for Reunion Measures for Phase Two Group

\begin{tabular}{llcc}
\hline & & $M$ & $S D$ \\
\hline Attachment anxiety & & 2.23 & 1.10 \\
Attachment avoidance & & 2.02 & 0.76 \\
Relationship satisfaction & & 6.31 & 0.77 \\
\hline Coping capacity & Well-rested & 5.48 & 1.22 \\
& General well-being & 5.71 & 1.07 \\
& Perceived relationship support & 6.32 & 0.92 \\
\hline Reunion experiences & Expectations met & 6.28 & 1.06 \\
& More emotionally connected & 4.94 & 1.35 \\
& More satisfied & 4.94 & 1.59 \\
& Things running more smoothly & 4.61 & 1.54 \\
& Quality of interactions & 5.81 & 1.35 \\
& Extent of conflict & 2.72 & 1.54 \\
\hline
\end{tabular}

Note. Response range $=1-7$ (see scale descriptions for specific value labels)

\section{Open-Ended Responses}

For the open-ended responses, I recruited an undergraduate student who was blind to the hypotheses (and all other variables including attachment dimensions) to code these responses. In addition, I acted as the reliability/validity check and coded a portion (30\%) of the main rater's responses. To assess and quantify the emotion/thought list, the student used Shaver and colleagues' (1987) emotion labelling taxonomy, consistent with Phase One (see Appendix J). To reiterate, when using this taxonomy the researcher's goal is to distil all emotion terms to these six basic terms (i.e., identifying which of the six cluster categories the listed emotion belongs 
to). In total, 41 participants had listed one emotion, 41 had listed two emotions, and 39 had listed three emotions. There was no relationship between attachment anxiety or avoidance and the number of emotions listed ( $r$ s equal to .18 and .07 respectively).

Prior to conducting my main analyses, I assessed the validity of the emotion/thought listing coding by assessing the extent of inter-rater reliability using percent agreement or Cohen’s Kappa (McHugh, 2012; Cohen, 1960). Cohen's Kappa (к) is similar to correlation coefficients in that its values range from -1 to 1 with 0 representing the amount of agreement that would be expected by chance. In this case, I used both estimates to assess inter-rater reliability for the first emotion/thought listing as recommended by McHugh. First, I calculated percent agreement by assigning the raters a 1 when their emotion coding matched, and 0 when they were mismatched. Then, I divided the number of ' 1 's assigned by the total number of subjects that listed an emotion/thought. This resulted in a perfect agreement for the first emotion/thought listing (100\% agreement). Further, the Cohen's Kappa estimate suggested near perfect agreement $(\kappa=1.00)$ as it takes into account chance agreement, which in this case was $85 \%$. Specifically, chance agreement for this variable was high given the little variation across emotion listings (with most listing 'joy' related emotions). Overall, these estimates suggest moderate to high agreement and a valid measure of primary emotions described.

\section{Results}

To assess the unique and interactive effects of attachment anxiety and attachment avoidance on the monthly diary outcomes (i.e., coping, relationship satisfaction, emotional support, quality of interactions), I used Multilevel Modelling (MLM) procedures. MLM procedures are ideal for analyzing nested data sets such as diary data (i.e., where repeated measures are nested within individuals). Prior to conducting any analyses, I first centered the 
attachment anxiety and attachment avoidance scores collected from the separation survey around the grand mean (Enders \& Tofighi, 2007). To conduct the following analyses, I used the SPSS mixed models platform (Painter) with participant treated as the level 1 predictor, and attachment anxiety, avoidance, and the interaction, as the level 2 predictors.

There were some main effects of attachment anxiety. Specifically, attachment anxiety was related to decreased coping capacity $[B=-.39, t(51)=-2.00, p=.050]$ and less relationship satisfaction $[B=-.29, t(48)=-3.01, p=.004]$. Next, there were a number of main effects of attachment avoidance. With respect to provision of emotional support within the relationship, attachment avoidance was related to feeling less satisfied with the emotional support received from one's partner $[B=-.43, t(48)=-2.04, p=.047]$, the tendency to push one's partner away when they provide support $[B=1.19, t(46)=5.13, p<.001]$, not liking to provide support to one's partner $[B=-.40, t(43)=-3.03, p=.004]$, and feeling more discomfort in expressing vulnerability to one's partner $[B=-1.13, t(47)=-6.05, p<.001]$. Attachment avoidance was also related to a decreased tendency to think about one's partner $[B=-.32, t(46)=-4.33, p<.001]$, not being as eager for one's partner's return $[B=-.21, t(46)=-2.97, p=.005]$, and not fantasizing about one's partner $[B=-.67, t(51)=-2.95, p=.005]$. Further, attachment avoidance was related to decreased quality of interactions with one's partner $[B=-.42, t(44)=-2.32, p=$ $.025]$.

To assess the unique and interactive effects of attachment anxiety and avoidance on the reunion outcomes, I conducted multiple linear regressions with the attachment scores collected in the reunion survey (as opposed to the separation survey for the prior analyses). Specifically, I used the mean-centered attachment anxiety, reunion mean-centered attachment avoidance, and interaction term as the predictors on the 10 reunion outcomes. Two-way interactions were 
assessed using simple slopes analyses (Aiken \& West, 1991), examining the effects of attachment anxiety at lower (-1 SD from the mean) and higher (+1 SD from the mean) levels of attachment avoidance.

There were significant main effects of attachment anxiety. Specifically, attachment anxiety was related to decreased relationship satisfaction, $[B=-.30, \beta=-.43, t(32)=-2.69, p=$ .011], however attachment anxiety was also related to reports that one is more satisfied with the relationship now (during the reunion) relative to the time before the deployment $[B=.59, \beta=$ $.48, t(32)=2.21, p=.034]$

There were also significant main effects of attachment avoidance. Specifically, attachment avoidance was related to feeling less well-rested $[B=-.98, \beta=-.59, t(37)=-2.74, p=$ $.009]$, less perceived relationship support $[B=-.56, \beta=-.44, t(37)=-2.98, p=.005]$, compromised general well-being $[B=-1.04, \beta=-.71, t(37)=3.77, p=.001]$, and lower quality interactions with one's partner $[B=-1.06, \beta=-.59, t(32)=-3.16, p=.003]$.

These main effects were qualified by significant two-way interactions between attachment anxiety and avoidance. First, there was a significant two-way interaction for relationship satisfaction, $B=-.21, \beta=-.28, t(32)=-2.11, p=.043$ (see Figure 17). Specifically, at low levels of attachment avoidance the relationship between attachment anxiety and relationship satisfaction was not significant, $B=-.14, \beta=-.20, t(32)=-0.97, p=.339$. However, at high levels of avoidance there was a significant negative relationship between attachment anxiety and relationship satisfaction, $B=-.46, \beta=-.66, t(32)=-3.75, p=.043$. 


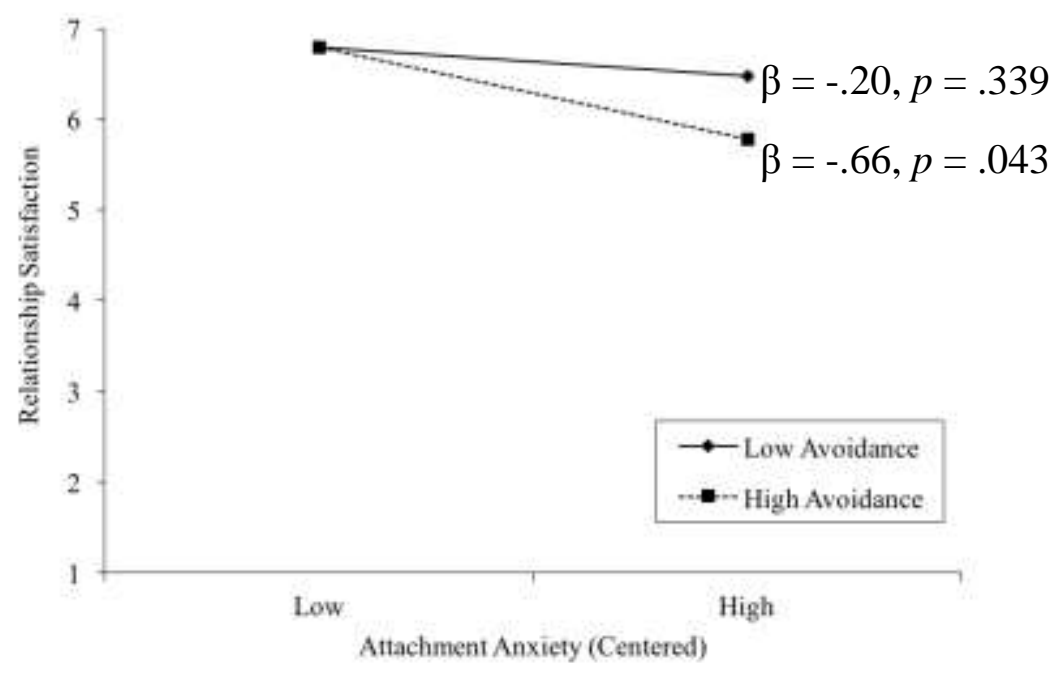

Figure 17. Interaction between attachment anxiety and attachment avoidance on relationship satisfaction.

Next, there was a significant two-way interaction for expectations met, $B=-.34, \beta=-.33$, $t(32)=-2.12, p=.042$. Similar to the previous analysis, at low levels of avoidance the relationship between attachment anxiety and expectations met was not significant, $B=-.01, \beta=-$ $.01, t(32)=-0.05, p=.958$ (see Figure 18). However, at higher levels of avoidance there was a significant negative relationship between attachment anxiety and expectations met, $B=-.54, \beta=$ $-.56, t(32)=-2.67, p=.012$.

There was a significant two-way interaction for feeling more versus less satisfied relative to the time before the deployment, $B=-.76, \beta=-.58, t(32)=-3.21, p=.003$ (see Figure 19). This time, at low levels of avoidance there was a significant positive relationship between attachment anxiety and feeling more satisfied relative to the time before the deployment, $B=$ $1.17, \beta=.95, t(32)=3.36, p=.002$. However, at high levels of avoidance the relationship between attachment anxiety and feeling more versus less satisfied relative to before the deployment was not significant, $B=.01, \beta=.01, t(32)=0.04, p=.971$. 


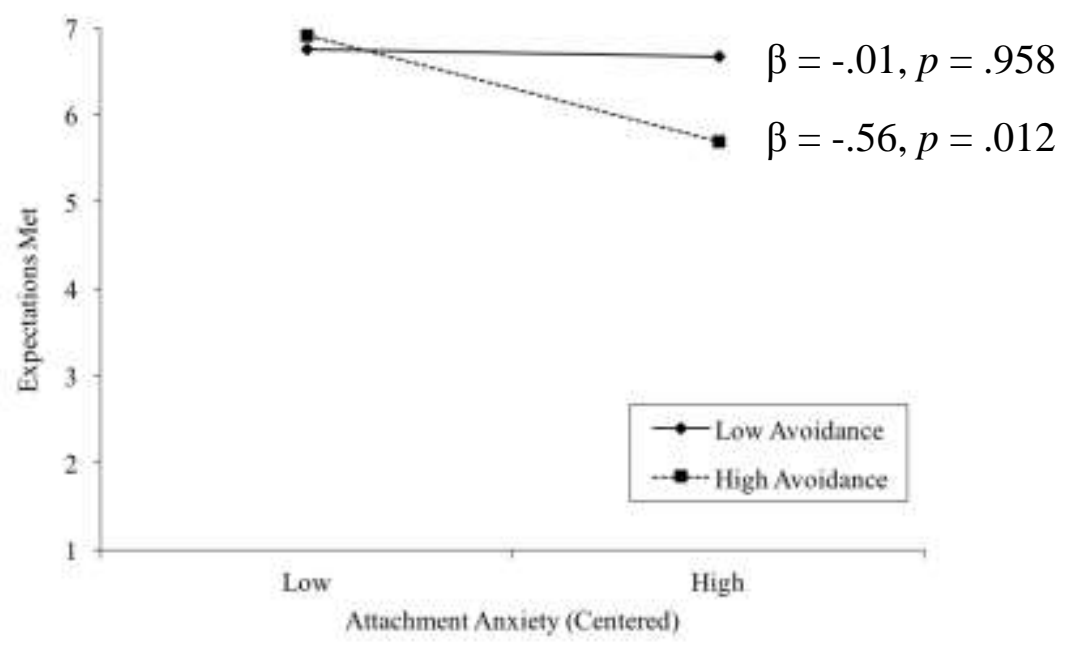

Figure 18. Interaction between attachment anxiety and attachment avoidance on the extent to which expectations of the reunion were met.

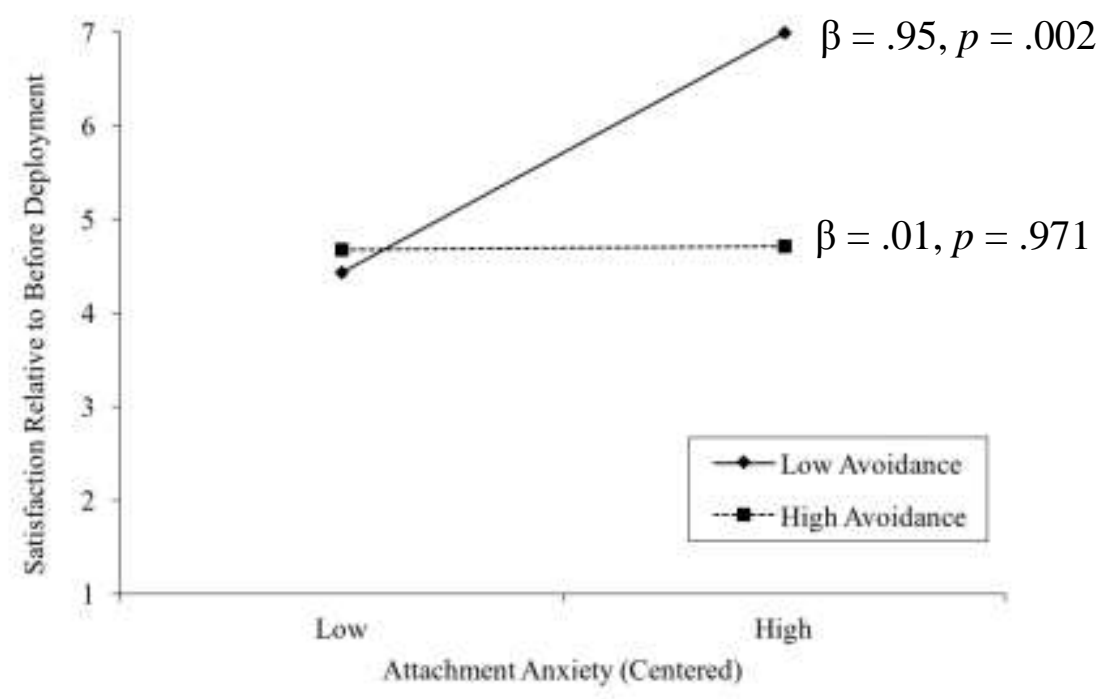

Figure 19. Interaction between attachment anxiety and attachment avoidance on feeling more versus less satisfied with one's relationship relative to the time before the deployment.

There was also a significant two-way interaction for feeling more versus less emotionally connected relative to the time before the deployment, $B=-.78, \beta=-.50, t(32)=-2.58, p=.015$ 
(see Figure 20). While neither of the simple slopes reached significance, the simple slope for low avoidance approached significance.

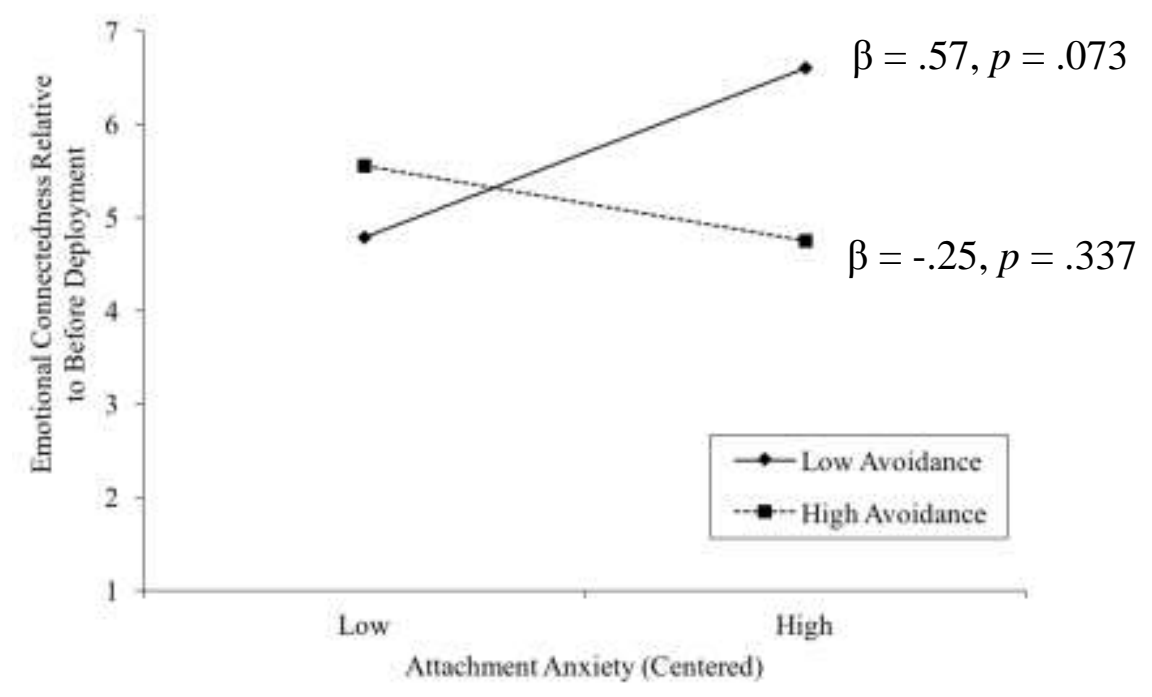

Figure 20. Interaction between attachment anxiety and attachment avoidance on feeling more versus less emotionally connected to one's partner relative to the time before the deployment.

Finally, there was a significant two-way interaction for feeling as though things are running more versus less smoothly around the house relative to before the deployment, $B=-.67$, $\beta=-.45, t(32)=-2.29, p=.029$ (see Figure 21). Again, neither of the simple slopes were significant however the simple slope for low avoidance approached significance.

In summary, for those higher in attachment avoidance, attachment anxiety was negatively related to relationship satisfaction and beliefs that one's expectations of the reunion were met. In contrast, for those lower in attachment avoidance, attachment anxiety was positively related to one's relationship satisfaction relative to before the deployment, and somewhat related to feeling more emotionally connected to one's partner, and that the household was running more smoothly, relative to before the deployment 


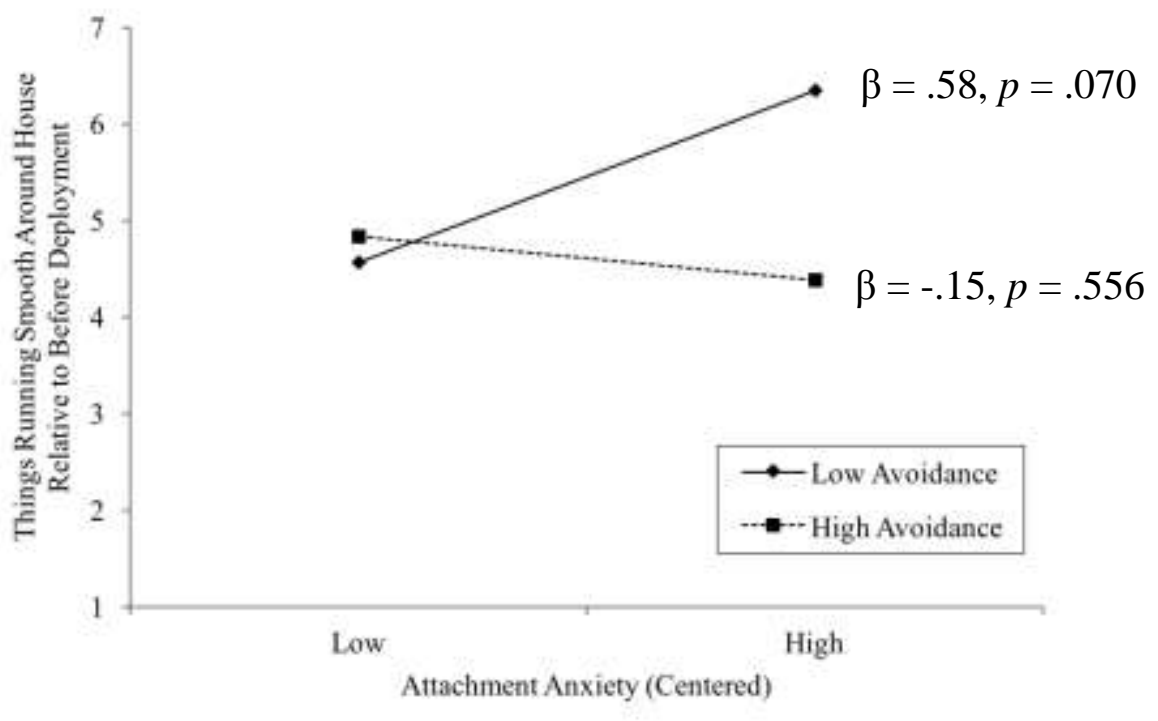

Figure 21. Interaction between attachment anxiety and attachment avoidance on feeling as though things are running more versus less smoothly around the house relative to the time before the deployment.

\section{Mediation Models}

Next, I was interested in testing mediation models to assess relationships between attachment orientations and perceptions of relationship and intrapersonal functioning during the ongoing separation. Although attachment anxiety wasn't predictive of many of the monthly indices, attachment avoidance was associated with a number of indices intrapersonal, and relationship indices. For this reason, the following analyses were focused on explaining some of the relationships between attachment avoidance and perceptions of emotional support provision and relationship and coping outcomes. Although MLM is an effective means to study relationships between variables that are clustered, nested, and have some degree of dependence, these same analyses cannot accurately or successfully accommodate testing of mediation models. In particular, models involving more than the assessment of level 1 predictors, mediators, and dependent variables (so the presence of at least one level 2 predictor) cannot be 
fit using MLM (see Preacher, Zhang, \& Zyphur, 2011). Given that I was interested in assessing relationships between level 2 predictors (attachment avoidance), and level 1 mediators (provision of emotional support) and outcomes (coping capacity and relationship satisfaction), using MLM procedures would not be appropriate. For this reason, I decided to calculate overall scores on each of the monthly measures and conduct more conventional tests of indirect effects using Preacher and Hayes (2008) bootstrapping method.

Specifically, I wanted to test whether (dis)comfort in expressing emotional vulnerability (a defining feature of attachment avoidance) predicted the tendency to push one's partner away when they are providing support, satisfaction with the emotional support received, (dis)like for providing support to one's partner, as well as coping capacity, relationship satisfaction, and quality of interactions during the separation (controlling for attachment anxiety and the two-way interaction term. The Preacher and Hayes's (2008) method was particularly appropriate given the small sample size $(n=50)$, as it does not make assumptions about the shape of the distribution of the indirect effect estimate circumventing issues of non-normality and low power (Preacher \& Hayes, 2004; Efron \& Tibshirani, 1993; Mooney \& Duval, 1993; Shrout \& Bolger, 2002). To reiterate, the bootstrapping method (here using 1000 samples) produces an empirically derived CI of the indirect effect estimate. If zero is not included in the CI, one can conclude that the indirect effect is significant. I performed these analyses using an SPSS multiple mediator macro (available from afhayes.com) that allows for the inclusion of control variables into the tests of indirect effects.

Unfortunately, I was not able to test any of these models, as attachment avoidance was not significantly related to most of the monthly aggregate measures including overall relationship satisfaction, coping capacity, and satisfaction with emotional support received. 
Attachment avoidance was only significantly related to the overall tendency to push one's

partner away $[B=.73, \beta=.51, \mathrm{t}(48)=2.96, p=.005]$ and being less likely to providing support to one's partner $[B=-.21, \beta=-.37, t(48)=-2.12, p=.039$. I do not have any reason to believe that one of these variables might be mediating the effects of the other, so I did not test any mediation models with these variables. ${ }^{13}$

\section{Open-Ended Responses}

In order to assess relationships between attachment anxiety and avoidance, and the nature of emotions listed at the time of the reunion, I performed a discriminant analysis. This analysis assesses whether predictor variables (in this case the centered anxiety, avoidance, and interaction terms) can reliably determine group membership, which in this case was the emotion described (out of the six previously outlined). In general, only emotion labels indicating joy, fear, love, anger, and sadness were reported (emotion labels related to surprise and anger were not reported). For the first emotion/thought listing, $90 \%$ of participants reported emotions indicative of joy, $7.5 \%$ fear, and $2.5 \%$ love. For the second emotion/thought listing $78 \%$ reported feeling joy, $19.5 \%$ fear, and $2.5 \%$ love. Finally, for the third emotion/thought listing $70 \%$ reported joy, $21 \%$ fear, $6 \%$ love, and $3 \%$ sadness. With this sample, there was more variation in the distribution of emotions described in each thought listing and more participants reported all three emotions $(n=33)$, so I am reporting results for the assessment of variation in reported emotions as a function of anxiety, avoidance, and their interaction for all three emotion/thought listings.

\footnotetext{
${ }^{13}$ I was originally interested in testing the trajectory of the relationships among attachment anxiety, attachment avoidance, and separation outcomes and seeing whether the relationship is consistent (individuals with higher levels of attachment anxiety or avoidance consistently report difficulties across the separation period) or if this relationship changes as a function of time in a linear or non-linear fashion. Unfortunately, only 31 spouses completed a minimum of 3 monthly surveys which would be required to test curvilinear patterns. Due to issues surrounding low power with this particular sample, I decided to not assess linear and curvilinear effects of attachment anxiety as a function of time. I was concerned about the generalizability and replicability of any observed effects if any were found. Also, the large scale survey did allow me to test such trends with adequate power.
} 
Unfortunately, for all three analyses, the assumption of homogeneity of group variances could not be assessed, as there were fewer than two non-singular group covariance matrices. This may be a result of too much shared variance between attachment anxiety and avoidance, too few cases, or nonnormality with the dependent variables. Regardless, I decided not to proceed with interpretation of the main analyses given potential issues with the group covariance matrices.

Participants also described in an open-ended fashion what they did when they saw their partner for the first time. These descriptions were coded by the same primary rater for the degree of physical intimacy described in the initial meeting. Specifically, descriptions were given a rating from 1-4, where 1 represented descriptions with little to no physical contact mentioned, 2 represented some physical intimacy mentioned (hugged or kissed), 3 represented a moderate amount of physical intimacy (a long embrace, kissed and hugged, ran into the partner's arms), and 4 assigned to descriptions that were high in physical intimacy (ran into the partner's arms plus a long embrace or kissing, saying "I love you", crying). This resulted in a variable that was fairly normally distributed, with $17 \%$ assigned a ' 1 ', $12 \%$ assigned a ' 2 ', $56 \%$ assigned a ' 3 ', and $15 \%$ assigned a ' 4 ' for the extent of physical intimacy in the initial meeting.

Similar to prior analyses, I was interested in assessing the extent to which levels of attachment anxiety, avoidance, or an interaction between anxiety and avoidance, are related to reported levels of physical intimacy in seeing one's partner for the first time after the deployment. In Phase One, I found that avoidance significantly predicted less intimacy reported during the reunion. Therefore, I predicted that attachment avoidance, more so than attachment anxiety, would be negatively related to lower levels of reported physical intimacy during the initial meeting. However, neither attachment avoidance, attachment anxiety, nor their interaction 
were significantly related to reports of physical intimacy during the reunion ( $p s$ ranging from $.17-.22)$.

\section{Discussion}

Consistent with previous findings (Collins \& Read, 1990; Fraley \& Shaver, 1998) and in line with the findings from Phase One, attachment anxiety was related to a decreased ability to cope and to decreased relationship satisfaction during the ongoing deployment (across four months). This is intuitive given that attachment anxiety is associated with strong desires for closeness and intimacy and their reliance on attachment figures for emotional regulation and support. However, I also predicted that attachment anxiety might be related to some difficulties providing emotional support to one's partner during the ongoing separation (Collins \& Feeney, 2000), but attachment anxiety was not predictive of indices of emotional support. I also predicted that attachment anxiety would be related to greater fantasizing about the reunion, given that highly anxious individuals are more dependent on their partners for validation and support in general, regardless of the context (e.g., Davilla, 2001; Wei, Mallinckrodt, Larson, \& Zakalik, 2005). With this particular sample, attachment anxiety and attachment avoidance were strongly correlated. Attachment avoidance uniquely predicted perceptions in both of these domains (emotional support and fantasizing), so it is possible that because avoidance was a better predictor, there was not enough unique variance of attachment anxiety to be able to effectively predict leftover variance in these indices once the relationship between anxiety and avoidance was controlled for. This study was also underpowered due to a low response rate among invited participants, so it is possible that the effects of attachment anxiety on these constructs might be distinguishable with larger and different samples with less of an overlap between levels of attachment anxiety and attachment avoidance. 
Consistent with the theorizing that attachment avoidance would be associated with deactivating strategies in response to environmental threats, I found that attachment avoidance was not related to indices of emotional well-being but was related a decreased tendency to think and fantasize about one's partner and their return. Further, in line with findings by Collins and Read (1990), attachment avoidance was related to lower quality partner interactions, and difficulties with the provision of emotional support. Specifically, attachment avoidance was related to a general discomfort in expressing vulnerability to one's partner and not enjoying the process of providing emotional support to one's deployed partner. In addition, attachment avoidance was related to less satisfaction with the emotional support received from one's partner, and to pushing one's partner away when they do provide support.

The next part of the study was a replication of the phase one reunion survey. In line with findings by Cafferty and colleagues (1994) and the reunion findings, attachment anxiety was related to decreased relationship satisfaction, but not to coping capacity. On the other hand, different from the reunion findings, attachment avoidance was now related to decreased coping capacity as assessed by all three indices (feeling well-rested, general well-being, perceived relationship support). This might be an indication of the breakdowns in coping abilities for highly avoidant individuals as observed by Mikulincer and Florian (1998). On the other hand, and similar to my explanation above, it is possible that due to a high degree of shared variance between the attachment constructs with this sample, attachment anxiety was not able to predict much unique variance once the effects of attachment avoidance were controlled for (and thus the relationship between attachment anxiety and avoidance controlled for). In line with previous research and the reunion findings, attachment avoidance was associated with lower quality partner interactions. 
It was interesting to find that those lower in attachment avoidance, attachment anxiety was associated with decreased relationship satisfaction, but also that one is more satisfied with the relationship now than before the deployment. Although seemingly contradictory, these findings are consistent with those observed by Sprecher (1999) that although participants who remained together over the course of a longitudinal study expressed a belief that their love and satisfaction increased over time, their actual ratings of love and satisfaction over time were not improving. Individuals with lower levels of avoidance desire higher levels of emotional and physical intimacy, so it is understandable that lower levels of avoidance coupled with higher levels of attachment anxiety (fears of abandonment, and in need of validation and reassurance) would be associated with greater satisfaction in being reunited with one's partner, and therefore assume that they must be more satisfied as a result. In other words, those higher in anxiety and lower in avoidance might believe that they are truly more satisfied with their relationship during the reunion than in the past, driven mainly by their concerns for closeness and intimacy which are now resolved.

Finally, although I predicted that attachment anxiety would be more related to perceptions that the reunion period has not lived up to one's original expectations, this relationship was true but only for those with higher levels of attachment avoidance. I should note that this sample was about half of the size of the Phase One sample ( $n=36$ and $n=68$ respectively). Therefore, it is possible that such two-way interactions are spurious in nature, and do not reflect accurate estimates of the parameters examined (Hinkle, Wiersma, \& Jurs, 2003). Tabachnick and Fidell (2007) recommend a minimum sample size of 104 plus a degree of freedom for each independent variable. In this case, I should have sampled a minimum of 107 cases to adequately estimate parameter estimates. 
For a number of reasons (restricted range of time of survey completion, lack of appropriate analyses given the type of data and low sample size), I was not able to adequately assess the trajectory (linear or curvilinear) of the relationships among attachment anxiety, attachment avoidance, and outcomes across the ongoing deployment as a function of time separated and time reunited. As such, this was one of the major goals of Phase Three. 


\section{Chapter 5}

\section{Phase Three - Large-Scale Survey}

The purpose of this third and final phase of the project was to examine how attachment dimensions moderated the impact of military life on spouses of military members in a large-scale survey. Specifically, I wanted to elucidate the relationships between attachment styles and spousal functioning on a number of variables indicating their psychological adjustment, coping strategies, and relationship perceptions, but also to replicate findings from the previous three phases. In particular, I was interested in testing whether the effects of attachment anxiety and avoidance might be exacerbated for those currently experiencing a deployment separation or reunion relative to those not experiencing a separation or reunion, and the potential trajectory of such outcomes as a function of separation or reunion length. This survey captured a number of indices of spouse's perceptions of their intrapersonal and interpersonal (relationship) functioning

for spouses of varying partner deployment status' (spouses of partners not currently deployed, deployed, preparing for a deployment, and those recently reunited with their partners from a deployment). Due to a more adequate sample size, and with this being an independent design (not repeated measures), I was also able to more easily assess the trajectory of the effects of attachment dimensions on intrapersonal and relationship outcomes as a function of time separated, and time reunited (linear and curvilinear functions). Finally, I was able to use mediation models to identity consistent and powerful mediators in the relationships between attachment orientations and intrapersonal and relationship outcomes. 


\section{Method}

\section{Participants and Procedure}

The third and final phase of this project consisted of a large-scale survey (titled 'Quality of Life Among Military Families: A Survey of Spouses and Partners of Canadian Forces Members') that was created and administered by DGMPRA and targeted to a sample of spouses/partners of CAF Regular Force members in $2012^{14}$. The population, all spouses or common-law partners of CAF Regular Force members, was divided into subsets (called strata) using stratified sampling before a sample of each of these subsets was selected. This method increases the precision of the overall population estimates by ensuring that the number of individuals selected from each stratum for the sample is proportional to the number of individuals in each stratum in the population of interest (Statistics Canada, 2003). A sampling frame was then extracted from the CAF administrative list of 39,104 CAF spouses. The sampling frame consisted of three sub-populations: (1) CAF spouses whose military partners were deployed $(N=1,435)$; (2) CAF spouses whose military partners were posted to the Joint Personnel Support Unit ${ }^{15}$ (JPSU: $N=529$ ); and (3) CAF spouses whose military partners were neither deployed nor posted to JPSU $(N=37,140)$. The final sample included all CAF spouses in the first two sub-populations and a stratified random sample of CAF spouses from the third. After excluding invalid home addresses, 9,359 CAF spouses were contacted. The survey was mailed to the home addresses of these 9,359 CAF spouses. Surveys could not be delivered to

\footnotetext{
${ }^{14}$ The survey population excluded spouses of (1) Officer Cadets; (2) personnel with less than one year of service; (3) personnel who are posted on foreign exchange; (4) untrained CAF personnel on the Basic Training List, the Subsidized University Training List, or trained CAF personnel who are not considered effective strength (retirement leave); and (5) personnel with no home address listed in the sampling frame.

15 The Joint Personnel Support Unit (JPSU) provides administrative support and programs to ill or injured CAF members of the Regular and Reserve Force who are experiencing a medical condition that limits them from performing their regular duties for more than six consecutive months (National Defence and the Canadian Armed Forces, 2015).
} 
1,062 home addresses, resulting in a final eligible survey sample of 8,297. A total of $1,895 \mathrm{CAF}$ spouses responded yielding an adjusted response rate of $23 \%$ (similar to the other phases). However, 177 participants completed less than $50 \%$ of the attachment key, so were excluded from relevant analyses. Therefore, the final sample consisted of 1,718 spouses.

Spouses were mostly women $(1,586$ or $93 \%)$ between the ages of 19 to $86(M=39.12$, $S D=9.17)$. Spouses had been in a common-law relationship or married to their partners for an average of 14.23 years $(S D=8.55)$, and most had a partner of the opposite sex $(99 \%)$. Most spouses had a university degree (28\%) or college diploma (24\%), most were employed full-time (51\%), and had children (77\%). A small number of spouses were CAF members (18\%) but the majority were civilian (82\%). The spouses had partners in the RCN (30\%), CA (39\%), and the RCAF (32\%). On average partners had experienced 1.60 deployments $(S D=3.48)$, but most were not currently deployed (89\%). For the spouses of deployed partners, their partners had been deployed an average of 6.62 months ( $S D=9.91$ months, range $=1-60$ months). For the spouses whose partners were not currently deployed, their partners had returned from their most recent deployment an average of 14.92 months ago ( $S D=22.14$ months), with $14 \%(\mathrm{n}=212)$ of partners having returned from a recent ( $<12$ months) deployment. Further, only $6 \%(\mathrm{n}=93)$ of these non-deployed partners were currently preparing for another deployment.

\section{Measures $^{16}$}

Attachment Anxiety and Avoidance

Attachment anxiety and avoidance were assessed using the ECR-R measure from Phases One and Two. In this sample, anxiety and avoidance were found to be reliable (Cronbach's $\alpha=$

\footnotetext{
${ }^{16}$ Prior to conducting any analyses, I requested the following measures from the lead internal researcher on the Quality of Life project. Therefore, the following measures are only a subset of the original survey that was administered. Measures were selected based on their perceived relevance to my research. Analyses were not conducted prior to my selection of relevant variables.
} 
.93 and .91 respectively), and were correlated, $r(1742)=.66, p<.001$ (see Table 6 for descriptive statistics). Impact of Military Life on Career

There were 6 items that captured the extent to which a partner's military career has impacted the participants' employment or career opportunities for better or worse (e.g., "I have made some employment or career sacrifices because of my partner's military career”, see Appendix K) from 1 (strongly disagree) to 5 (strongly agree). This was not an established scale, but given that many items seem to be measuring related constructs I decided to conduct EFA procedures in line with Fabrigar and Wegener (2012) to assess whether the items were capturing a similar latent construct and could therefore be combined into an aggregate score.

To determine the appropriate number of common factors, I first examined the scree plot based on eigenvalues from the reduced matrix (see Figure 22). According to this plot, there is a good case for a one-factor model, with the only visible drop in eigenvalues occurring after the first factor (from 2.16 to .18).

As a secondary test for determining the appropriate number of common factors, I performed a parallel analysis using the following parameters: $\mathrm{N}=1895$, number of variables $=6$, number of datasets $=100$, and confidence interval $=.95$. According to this analysis, only one factor had an eigenvalue greater than those produced by random data, either by the mean or $95^{\text {th }}$ percentile values. Therefore, according to this analysis, and consistent with the scree plot, a onefactor solution seems appropriate. 


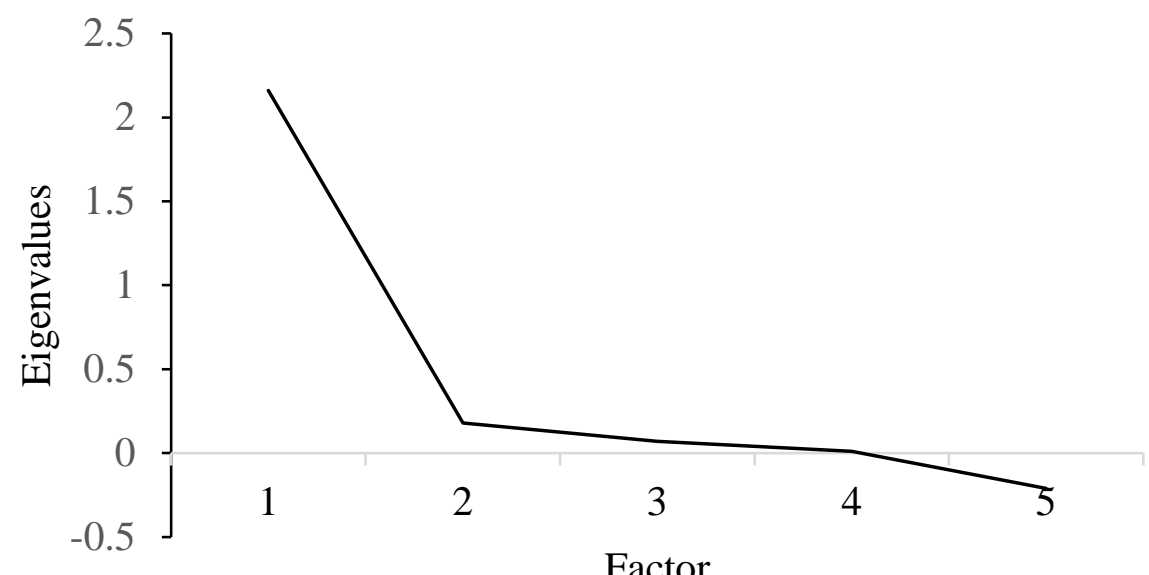

Figure 22. Scree plot of the reduced correlation matrix for impact of military life on career items (Phase Three).

The final method I used to determine the appropriate number of factors was assessing model fit using the root mean square error estimates for the one, three, and four-factor models. The one-factor model showed a marginal fit (RMSEA $=.102)$ and the two-factor model showed an acceptable fit (RMSEA =.062). In order to better determine how many common factors would be appropriate, I examined the interpretability of the factor loadings for the one and twofactor models, by performing an EFA using ML as the extraction method and using an oblique rotation.

To summarize the findings from both models, the two-factor model had one low communality, and two complex loadings. The one-factor model also had one communality, and one variable that loaded weakly on the factor (factor loading $=.25$ ) which was "I am underqualified for the work I am doing because of my spouse/partner's military career". Given the results from the scree test and parallel analysis, and to reduce the problems associated with cross-loadings (shared variance across factors), I decided to retain the one-factor model. This factor explained $36.08 \%$ of variance in measures (Eigenvalue $=2.16$ ), was labelled military life 
impacting career. When I performed a reliability analysis, the problematic item if removed would only improve the overall alpha by .03. For this reason, I decided to include all items in the overall scale, of which the reliability was acceptable (Cronbach's $\alpha=.76$ ). Overall scores for the implications of military life on one's career were calculated using the mean of all items, after reverse scoring relevant items, with higher scores reflecting that military life has had a more positive impact one one's career (see Table 6 for descriptive statistics).

\section{Stress During Military Experiences}

Spouses were asked to indicate how stressful they found 10 different military experiences to be if they had experienced that scenario in the past 12 months (e.g., "A combat-related deployment" and "Uncertainty about future deployments", see Appendix L) from 1 (not at all stressful) to 4 (very stressful). In order to assess whether items were measuring a similar and broader construct (and could therefore be aggregated), I performed EFA procedures similar to previous scales and in line with recommendations by Fabrigar and Wegener (2012).

To determine the appropriate number of common factors, I first examined the scree plot based on eigenvalues from the reduced matrix (see Figure 23). According to this plot, there is a good case for a two-factor model, with the final visible drop in eigenvalues occurring after the second factor (from 1.04 to .30).

As a secondary test for determining the appropriate number of common factors, I performed a parallel analysis using the following parameters: $\mathrm{N}=1895$, number of variables $=$ 10 , number of datasets $=100$, and confidence interval $=.95$. According to this analysis, two factors had eigenvalues greater than those produced by random data, either by the mean or $95^{\text {th }}$ percentile values. Therefore, according to this analysis, and consistent with the scree plot, a twofactor solution seems appropriate. 


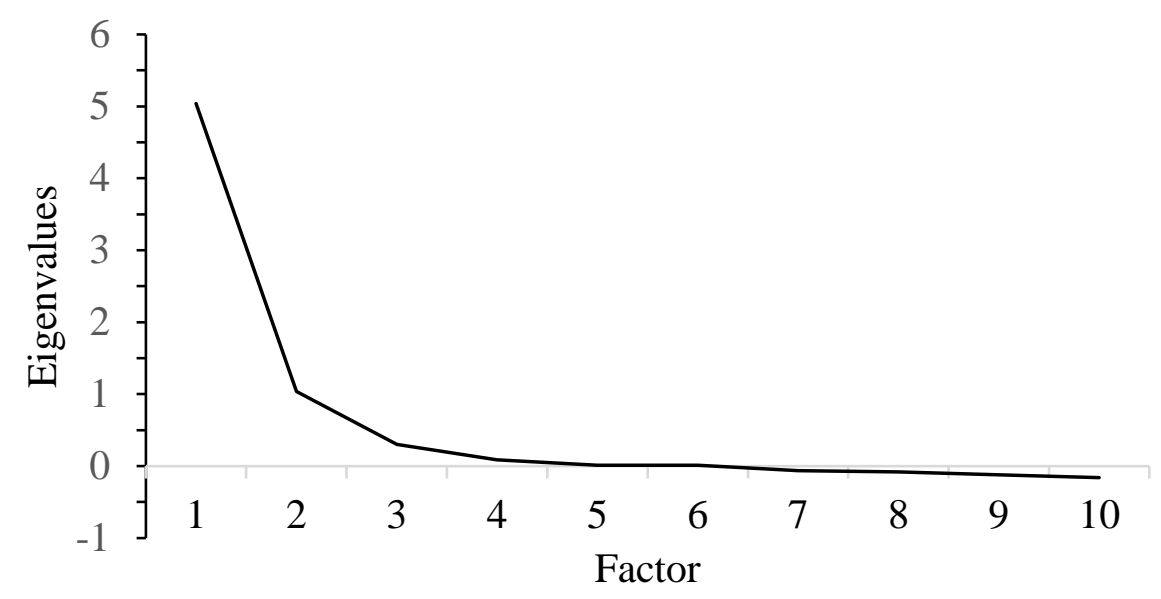

Figure 23. Scree plot of the reduced correlation matrix for stress during military experiences items (Phase Three).

The final method I used to determine the appropriate number of factors was assessing model fit using the root mean square error estimates for the one, three, and four-factor models. The one-factor model showed an acceptable fit $($ RMSEA $=.066)$ and the two, three, and fourfactor models showed a close fit (RMSEAs $=.036, .023$, and .008 respectively). To summarize, the two-factor model seemed appropriate given the findings from the scree test, parallel analysis, and the RMSEA index. In order to assess the interpretability of the two-factor model, I performed an EFA using ML as the extraction method and using an oblique rotation. There were no low communalities, and no complex loadings.

The first factor $($ Eigenvalue $=5.04)$ explained $50.4 \%$ of the variance across measures and reflected stress experienced across general military experiences including a partner's military duties, deployments, and juggling military and family life (6 items: e.g., "Difficulty balancing demands of family life and your spouse/partner's military duties" and "Intensified training schedule for your spouse/partner"). The 6 items were aggregated by taking the mean of all items, with higher scores reflecting more stress experienced during general military experiences. This 
scale's reliability was good (Cronbach's $\alpha=.90)$ and no items would improve the scale if removed.

The second factor explained $10.4 \%$ of the variance across measures (Eigenvalue $=1.04$ ) and reflected stress experienced as a result of injuries or a combat-related deployment (4 items: e.g., "caring for your ill, injured, or disabled spouse/partner"). The 4 items were aggregated by taking the mean of the respective items, with higher scores indicating greater stress experienced as a result of partner injury or combat deployment. This scale had good reliability (Cronbach's $\alpha$ $=.87$ ) and no items would improve this result if removed. The two factors were moderately correlated (factor correlation coefficient $=.59$ ) suggesting an oblique rotation was appropriate Descriptive statistics for the factors and single item can be found in Table 6.

Stress

Four items tapped into the amount of stress that spouses are currently experiencing (e.g., "Thinking about the amount of stress in your life, would you say that most days are", see Appendix M) and each item was associated with a response scale from 1 (e.g., Not at all stressful) to 5 (e.g., Extremely Stressful). I performed an EFA on the items to see whether they were capturing a broader construct and could therefore be aggregated, using procedures similar to previous scales and in line with recommendations by Fabrigar and Wegener (2012). To determine the appropriate number of common factors, I first examined the scree plot based on eigenvalues from the reduced matrix (see Figure 24). According to this plot, a one-factor model seems appropriate, the only visible drop in eigenvalues occurring after the first factor (from 1.30 to .08). 


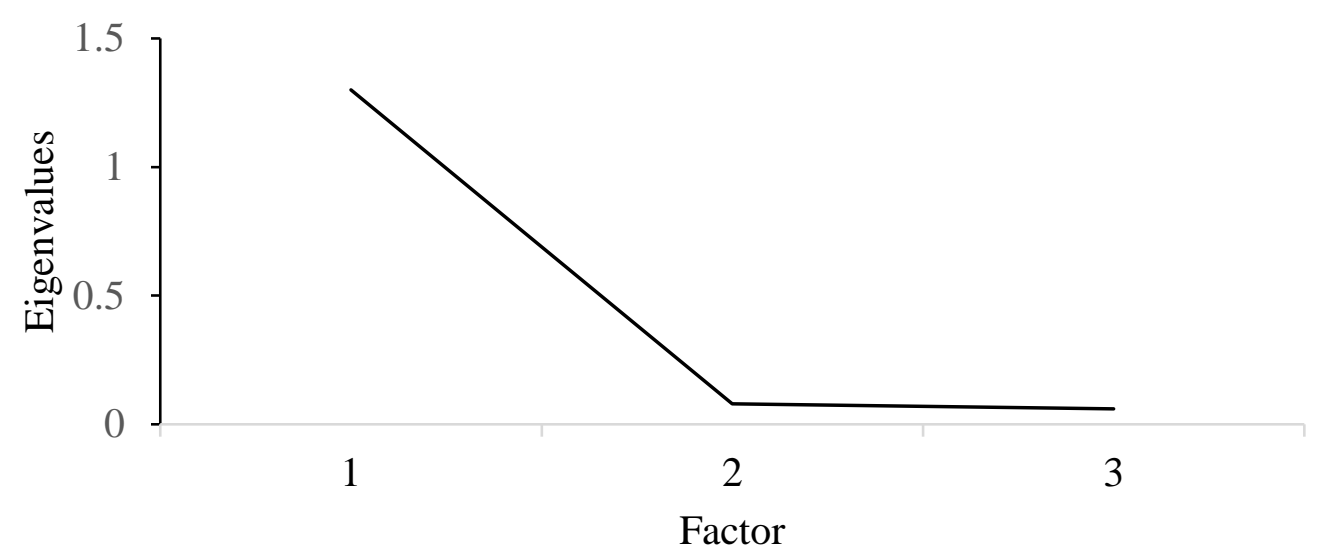

Figure 24. Scree plot of the reduced correlation matrix for stress items (Phase Three).

As a secondary test for determining the appropriate number of common factors, I performed a parallel analysis using the following parameters: $\mathrm{N}=1895$, number of variables $=$ 10 , number of datasets $=100$, and confidence interval $=.95$. According to this analysis, two factors had eigenvalues greater than those produced by random data, either by the mean or $95^{\text {th }}$ percentile values. Therefore, while the scree plot indicates that a one-factor solution might be appropriate, these results suggest that a two-factor solution might also be considered appropriate.

The final method I used to determine the appropriate number of factors was assessing model fit by computing the root mean square error estimates for the one-factor model, which showed an acceptable fit (RMSEAs $=.053)$. To summarize, the scree test and RMSEA index support a one-factor model, although results from the parallel analysis suggested that two factors might be appropriate. In order to assess the interpretability of the one-factor model, I performed an EFA using ML as the extraction method and using an oblique rotation.

The factor explained $32.4 \%$ of variance in measures (Eigenvalue $=1.30$ ) and had two items with low communalities (one as low as .008). In examining the factor loadings, three measures had strong loadings (between -.32-.99), but one variable "Do you have the desire to eat when stressed?" did not load strongly (loading = -.09). For this reason, I decided to aggregate the 
three items together, into an overall ability to handle daily demands measure by taking the mean of the two items, with the exception of eating when stressed which was left as a separate measure. Descriptive statistics for the factor and items are listed in Table 6.

\section{Subjective Well-Being}

The Satisfaction With Life Scale (SWLS: Diener, Emmons, Larsen, \& Griffin, 1985; Pavot \& Diener, 1993) was used to assess subjective well-being or life satisfaction. This scale contains 5 items (e.g., "In most ways my life is close to my ideal", see Appendix N) and participants are to indicate their agreement with these items on a scale from 1 (strongly disagree) to 7 (strongly agree). In this sample, the reliability was excellent (Cronbach's $\alpha=.91$ ) and total scores were calculated by taking the mean of all five items with higher scores indicating better subjective well-being or greater life satisfaction. Descriptive statistics for subjective well-being are listed in Table 6.

\section{Psychological Distress.}

The Kessler Psychological Distress Scale (K10: Kessler et al., 2002) was used to assess psychological distress, specifically the extent of anxiety and depressive symptoms experienced in the most recent four-week period. This scale contains 10 items (e.g., "During the last 30 days, about how often did you feel so sad that nothing could cheer you up?", see Appendix O) and respondents are to indicate the extent to which they experienced that symptom from 1 (none of the time) to 5 (most of the time). This scale showed good reliability with this sample (Cronbach's $\alpha=.90)$. Overall distress scores are calculated by summing responses to all items, with total scores ranging from 10 to 50. This scale is typically used as a screening instrument for clinicians: scores under 20 indicate the patient is well, scores from 20-24 indicate a likely mild mental disorder, scores from 25-29 indicate a likely moderate mental disorder, and scores over 
30 indicate a likely severe mental disorder. Kessler and colleagues report that about $13 \%$ of the adult population will score 20 and over, and about $25 \%$ of patients seen in primary care score 20 and over. Descriptives for psychological distress are listed in Table 6.

Mastery

The Pearlin Mastery Scale (PM: Pearlin \& Schooler, 1981; Pearlin, Lieberman, Menaghan, \& Mullan, 1981) was used to assess spouse's degree of mastery or the extent to which they perceive life events as being under one's control or a matter of fate. Mastery is seen as a psychological resource that can help individuals cope in the face of environmental stressors. This scale contains 7 items (e.g., "I often feel helpless in dealing with the problems of life", see Appendix P) and respondents are asked to indicate their agreement with these statements from 1 (strongly disagree) to 4 (strongly agree). Scores for mastery were calculated by taking the mean of the respective items after reverse scoring relevant items, with higher scores reflecting greater perceived mastery over life events. This scale showed good reliability (Cronbach's $\alpha=.81$ ). Descriptives for mastery scores are listed in Table 6.

\section{Coping Strategies}

The Brief COPE scale (Carver, 1997) was used to assess the various ways that spouses cope with disparate stressors. This scale contains 28 items that cover 14 coping strategies (2 items for each strategy, see Appendix Q). Respondents indicate the extent to which they use each strategy on a scale from 1 (not at all) to 4 (a lot). The 14 strategies are self-distraction (e.g., "I've been turning to work or other activities to take my mind off things"), active coping (e.g., "I've been concentrating my efforts on doing something about the situation I'm in"), denial (e.g., "I've been saying to myself "this isn't real."'), substance use (e.g., "I've been using alcohol or other drugs to make myself feel better"), use of emotional support (e.g., "I've been getting 
emotional support from others"), use of instrumental support (e.g., "I've been getting help and advice from other people"), behavioral disengagement (e.g., "I've been giving up trying to deal with it"), venting (e.g., "I've been saying things to let my unpleasant feelings escape"), positive reframing (e.g., "I've been trying to see it in a different light, to make it seem more positive), planning (e.g., I've been trying to come up with a strategy about what to do), humor (e.g., "I've been making jokes about it"), acceptance (e.g., "I've been accepting the reality of the fact that it has happened"), religion (e.g., "I've been trying to find comfort in my religion or spiritual beliefs"), and self-blame (e.g., "I've been criticizing myself"). Scores for the 14 strategies are calculated by taking the mean of the respective two items, with higher scores reflecting greater use of that strategy during stressful events.

Although a researcher may choose to analyze these strategies separately, Carver (2007) also suggests creating second-order factors from the 14 strategies. Given the large number of inter-correlations among scales (each strategy was correlated with at least seven other strategies), I indeed decided to perform EFAs consistent with previous scales and see whether there might be second-order factors that could explain such correlations.

To determine the appropriate number of common factors, I first examined the scree plot based on eigenvalues from the reduced matrix (see Figure 25). According to this plot, a threefactor model seems most appropriate, as the final most visible drop in eigenvalues occurring after the third factor (from .50 to .24 ). 


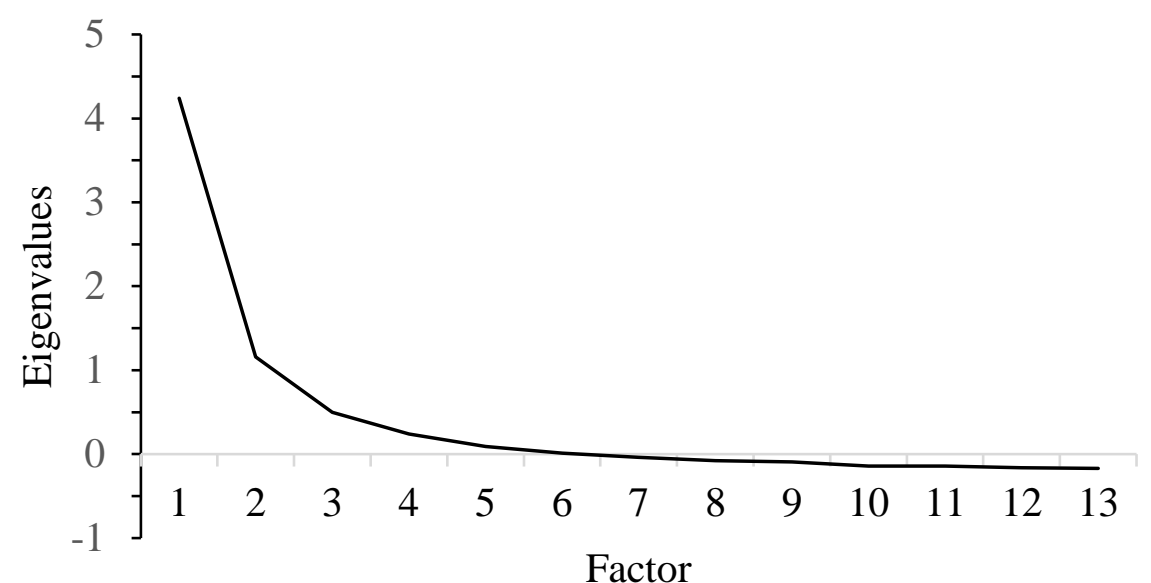

Figure 25. Scree plot of the reduced correlation matrix for coping strategies items (Phase Three).

As a secondary test for determining the appropriate number of common factors, I performed a parallel analysis using the following parameters: $\mathrm{N}=1895$, number of variables $=$ 14 , number of datasets $=100$, and confidence interval $=.95$. According to this analysis, two factors had eigenvalues greater than those produced by random data, either by the mean or $95^{\text {th }}$ percentile values. Therefore, while the scree plot indicates that a three-factor solution might be appropriate, these results suggest that a two-factor solution might also be considered appropriate.

The final method I used to determine the appropriate number of factors was assessing model fit by computing the root mean square error estimates for the one, two, and three-factor models. The one-factor model showed a poor fit $($ RMSEA $=.121)$, the two-factor model showed a marginal fit $(\mathrm{RMSEA}=.963)$, however the three-factor model showed an acceptable fit $($ RMSEA $=.0633)$. To summarize, the scree test and RMSEA index support a three-factor model, although results from the parallel analysis suggested that two factors might be appropriate. In order to assess the interpretability of the three-factor model, I performed an EFA using ML as the extraction method and using an oblique rotation. 
The three-factor model contained three items that had low communalities (.11-.22) suggesting weak inter-item correlations for these measures (religion, substance-use, humour). The first factor accounted for $30.3 \%$ of the variance in measures (Eigenvalue $=4.24$ ), was labeled active coping and reflected individuals using strategies aimed at addressing the stressor and managing the impact of that stressor. There were five strategies that loaded highly on this factor alone (communalities $>$.30) which were positive reframing, acceptance, planning, active coping, and using humour. This scale demonstrated good reliability (Cronbach's $\alpha=.82$ ), and no items would improve reliability if removed. The second factor accounted for $8.3 \%$ of the variance in measures $($ Eigenvalue $=1.16)$ and was labeled passive coping, reflecting the use of strategies aimed at avoiding confronting the stressor and managing it effectively. Specifically, the following four strategies loaded highly on this factor alone: behavioural disengagement, selfblame, denial, and substance use. The reliability for this scale was questionable, but no items would improve this value if removed (Cronbach's $\alpha=.61$ ). For this reason, I decided to leave the four passive coping strategies as separate measures. The third factor accounted for $3.5 \%$ of variance in measures (Eigenvalue $=0.50)$ and clearly reflected use of social support, with instrumental support, emotional support, and venting all loading highly on this factor alone. This scale had good reliability and no items would improve it if removed (Cronbach's $\alpha=.81$ ). There were two problematic items that I decided to leave as separate measures. The use of religion did not load highly on any factor (communalities between .01 to .27), and selfdistraction loaded highly on both active and passive coping factors (communalities $=.42$ and .30 respectively). Therefore, as a result of this analysis I settled on eight coping measures. Descriptive statistics for the two factors and six items are provided in Table 6. 


\section{Perceived Support and Resiliency}

Perceived support and resiliency was assessed using Bowen and Martin's (2011) Support and Resiliency Inventory for Spouses. Specifically, this measure examines military spouse's perceptions of support in their lives, their individual fitness, positive behaviours towards self and others, and their success in adapting to life challenges (military and otherwise). While spouses completed the full version, which contains 109 items that capture 37 dimensions, I selected to examine only 10 of these dimensions that seemed most relevant to my research, captured with a total of 30 items (see Appendix R). Responses to all items were indicated on a scale from 1 (strongly disagree) to 6 (strongly agree). In their own work, Bowen and Martin (2011) report these scales to be valid and reliable with good to excellent indices of internal consistency. The first dimension is relationship support and reflects feeling valued, cared for, and supported by one's partner (3 items: e.g., "I receive support and encouragement from my spouse/partner"). This scale had excellent reliability with this sample (Cronbach's $\alpha=.91$ ) and no items would improve it if removed. The second dimension is physical fitness and captures the extent to which a spouse is maintaining a healthy and active lifestyle (3 items: e.g., "I exercise on a regular basis"). The reliability for this scale was good and would not improve if items were deleted (Cronbach's $\alpha=.87$ ). The third dimension is emotional fitness and reflects an optimistic outlook and being able to enjoy life ( 3 items: e.g., "I look forward to beginning each day"). The reliability for this scale was excellent, and removing items would not improve it (Cronbach's $\alpha=$ .90). The fourth dimension is cognitive fitness and captures the use of planning and critical thinking when facing adversity ( 3 items: e.g., "When I face adversities, I think carefully about the consequences of my response before I act”). The reliability for this scale`s scores was good and would not be improved with items removed (Cronbach's $\alpha=.88$ ). The fifth factor is social 
fitness capturing the extent to which a spouse is willing to solicit and accept support from others in the community ( 3 items: e.g., "I am willing to turn to other people in the community for help or assistance, if I need it). This scale had good reliability which would not improve with the deletion of any items (Cronbach's $\alpha=.78$ ). The sixth factor called ethical fitness reflected the extent to which spouses lived life and face challenges according to a guiding set of principles or beliefs ( 3 items: e.g., "I draw strength from a guiding set of principles or beliefs when I face life challenges"). The reliability for this scale was excellent and could not be improved with items removed (Cronbach's $\alpha=.95)$. The seventh dimension life readiness reflects feeling capable of handling life challenges and daily matters, particularly when one's partner is not available (3 items: e.g., "I know where to turn for assistance or support if my spouse/partner is unavailable"). The reliability for this scale was acceptable (Cronbach's $\alpha=.74$ ) and no items would improve this scale if deleted. The eighth dimension called partner support reflected the extent to which spouses feel they are supportive of their partner and their military career ( 3 items: e.g., "I am supportive of my spouse/partner in or working for the military"). This scale's reliability was good and wouldn't improve with any items deleted (Cronbach's $\alpha=.81$ ). The ninth dimension called coping success captured whether spouses felt that they could cope with life stressors (3 items: e.g., "I confront the problem directly when I face a challenge or difficulty"). The reliability for this scale was good, and wouldn't improve with items removed (Cronbach's $\alpha=$ .84). Finally, the $10^{\text {th }}$ dimension relationship success assessed the extent to which spouses are happy with their relationships and committed to making the relationship successful (3 items: e.g., "I am committed to making my relationship with my spouse/partner a success"). The reliability for this scale was excellent (Cronbach's $\alpha=.90$ ) that couldn't be improved with any 
items deleted. Scores for all 10 dimensions were calculated by taking the mean of the respective three items (see Table 6 for descriptive statistics).

\section{Relationship Satisfaction}

There were two scales used to assess relationship satisfaction. The first scale is called the Quality of Marriage Index (QMI: Norton, 1983) and contains 6 items ${ }^{17}$ that assess relationship quality (e.g., "Our marriage/relationship is strong”, see Appendix S) on a scale from 1 (strongly disagree) to 7 (strongly agree). The reliability for this scale was excellent (Cronbach's $\alpha=.97$ ) and this would not improve with any items deleted. Scores for relationship satisfaction (QMI) were calculated by taking the mean of all items, with higher scores reflecting greater relationship satisfaction (see Table 6 for descriptive statistics).

The second scale used to assess relationship satisfaction was the Revised Dyadic Adjustment Scale (RDAS: Busby, Christensen, Crane, \& Larsen, 1995), which contains three subscales ${ }^{18}$ that are captured using a total of 14 items (see Appendix T). The first subscale is dyadic consensus, which asks spouses to rate the extent to which they agree with their partner on six important matters (6 items: e.g., “demonstrations of affection”) on a scale from 1 (always disagree) to 6 (always agree). The reliability for this scale was acceptable, and couldn't be improved with the removal of any items (Cronbach's $\alpha=.76$ ). Scores were calculated by taking the mean of the respective items, with higher scores reflecting greater perceived agreement on important issues (thus more satisfaction). The second subscale is dyadic satisfaction capturing

\footnotetext{
${ }^{17}$ In its original form, the first five items of the QMI are answered on a 1-7 scale, and the sixth item (degree of happiness in the relationship) on a 1-10 scale. However, responses for each item are typically standardized prior to calculating overall scores to make the scales somewhat equivalent. Norton (1983) reports this scale to be reliable, with uniformly strong inter-item correlation coefficients. For ease of responding and calculating scores, a 1-7 scale was used for all 6 items here.

${ }^{18}$ Results from an EFA using principal axis factoring and an oblique rotation confirmed the presence of 3 clear factors that were moderately correlated with one another ( $r$ s between .55-.64), validating the use of the three subscales.
} 
the degree to which spouses feel satisfied with their partner (4 items: e.g., "How often do you discuss or have you considered divorce, separation, or terminating your relationship?") on a scale from 1 (never) to 6 (all the time). This scale's reliability was good, and could not be improved with any items deleted (Cronbach's $\alpha=.82$ ). Dyadic satisfaction scores were calculated by calculating the mean of all items reverse-scored such that higher scores reflect greater dyadic satisfaction (thus greater relationship satisfaction). The final subscale dyadic cohesion contains 4 items, one item that asks spouses how often they participate in outside activities together from 1 (never) to 5 (everyday), and three items that ask spouses how often they engage in more specific activities (e.g., "have a stimulating exchange of ideas") from 0 (never) to 5 (more often). These four items were first transformed into z scores to standardize the response scales. Then, cohesion scores were calculated by taking the mean of the respective items standardized given their different response scales, and then mean scores were calculated with higher scores indicating greater cohesion. This scale's reliability was acceptable, and could not be improved with the removal of any items (Cronbach's $\alpha=.79)$. Descriptives for all three subscales are reported in Table 6. 
Table 6

Descriptive Statistics for Quality of Life Measures

\begin{tabular}{|c|c|c|c|c|}
\hline & & $M$ & $S D$ & $\alpha$ \\
\hline Attachment anxiety $^{1}$ & & 2.50 & 1.10 & .93 \\
\hline Attachment avoidance ${ }^{1}$ & & 2.37 & 0.95 & .91 \\
\hline Military life impacting career ${ }^{2}$ & & 2.72 & 0.97 & .76 \\
\hline Stress during general military experiences ${ }^{3}$ & & 2.47 & 0.82 & .90 \\
\hline $\begin{array}{l}\text { Stress during partner injury or combat } \\
\text { deplovment }\end{array}$ & & 2.36 & 1.00 & .87 \\
\hline \multirow[t]{2}{*}{ Stress $^{2}$} & Ability to handle daily demands & 3.25 & 0.71 & - \\
\hline & Desire to eat when stressed & 2.97 & 1.13 & - \\
\hline Subjective well-being ${ }^{1}$ & & 4.80 & 1.41 & .91 \\
\hline Psychological distress ${ }^{4}$ & & 17.8 & 6.77 & .90 \\
\hline Mastery $^{3}$ & & 3.00 & 0.54 & .81 \\
\hline \multirow[t]{8}{*}{ Coping strategies $^{3}$} & Active coping factor & 2.51 & 0.64 & .82 \\
\hline & Social support factor & 2.26 & 0.75 & .81 \\
\hline & Self-blame & 1.83 & 0.83 & - \\
\hline & Denial & 1.23 & 0.50 & - \\
\hline & Substance use & 1.21 & 0.54 & - \\
\hline & Behavioural disengagement & 1.38 & 0.70 & - \\
\hline & Self-distraction & 2.40 & 0.82 & - \\
\hline & Religion & 1.63 & 0.92 & - \\
\hline \multirow[t]{10}{*}{ Perceived support and resiliency ${ }^{5}$} & Relationship support & 5.38 & 0.93 & .91 \\
\hline & Physical fitness & 4.54 & 1.02 & .87 \\
\hline & Emotional fitness & 4.62 & 0.94 & .90 \\
\hline & Cognitive fitness & 4.89 & 0.72 & .88 \\
\hline & Social fitness & 3.51 & 1.18 & .78 \\
\hline & Ethical fitness & 4.88 & 1.17 & .95 \\
\hline & Life readiness & 4.95 & 0.83 & .74 \\
\hline & Partner support & 5.33 & 0.76 & .81 \\
\hline & Coping success & 4.51 & 0.88 & .84 \\
\hline & Relationship success & 5.42 & 0.84 & .90 \\
\hline Relationship satisfaction (QMI) $^{1}$ & & 5.89 & 1.26 & .97 \\
\hline \multirow[t]{3}{*}{ Relationship satisfaction (RDAS) } & Dyadic consensus $^{5}$ & 4.89 & 0.66 & .76 \\
\hline & Dyadic satisfaction $^{5}$ & 4.89 & 0.69 & .82 \\
\hline & Dyadic cohesion $^{6}$ & 0.00 & 0.79 & .79 \\
\hline $\begin{array}{l}{ }^{1} \text { Response range }=1-7 \text { (see scale descriptic } \\
{ }^{2} \text { Response range }=1-5 \\
{ }^{3} \text { Response range }=1-4 \\
{ }^{4} \text { Response range }=10-50 \\
{ }^{5} \text { Response range }=1-6 \\
{ }^{6} \text { This is an aggregate of standardized scores }\end{array}$ & as for specific value labels) & & & \\
\hline
\end{tabular}




\section{Results}

Prior to conducting my main analyses, I examined the distributional properties of my attachment dimensions including skewness, kurtosis, and the presence of outliers as such issues can inflate and distort relationships between variables. Although there are no established guidelines with respect to acceptable kurtosis and skewness values, West, Finch, and Curran (1995) recommend conducting normality transformations on variables that have skewness values above $|2|$ and/or kurtosis values greater than 7. The skewness (.92 and .71) and kurtosis values (.76 and -.128) for anxiety and avoidance were within this acceptable range. I then checked for the presence of univariate outliers for anxiety and avoidance, specifically values in excess of $z=$ -/+ 3.29 (Tabachnick \& Fidell, 2007). There were 10 cases that had avoidance $z$ scores in excess of 3.29, and three cases with anxiety $z$ scores in excess of 3.29 ( 1 of these cases had outstanding values for both variables). Therefore, there were 12 cases that had excess values on at least one of the attachment dimensions. For univariate outliers, Tabachnick and Fidell (2007) recommend changing the outliers such that they are still deviant scores, but just are not as deviant as before. Indeed, Osborne (2001) has shown that treatment or removal of outliers can reduce the probability of Type I and Type II errors. One way to handle outliers is the winsorized mean method whereby the outlying scores are replaced by the next-to-highest value, or the next-tooutlying value. In this case, the high scores would be replaced by the value closest to, but not above a $z$ score $=3.29$. The avoidance score (in 7 -scale units) closest to a $z$-score $=3.29$ was 5.44. Therefore, I inputted an avoidance score of 5.44 for the 10 extreme cases. Next, the closest anxiety score within the stated limits was 6 , so 3 cases were assigned a value of 3 for anxiety.

Given the number of analyses that I performed in Phase Three, and in order to make the following results more comprehensible, I decided to decompose all significant interactions yet 
only incorporate figures for those with significant simple slopes in the thesis body. Figures depicting interactions with non-significant simple slopes are presented in Appendices.

\section{Attachment Anxiety and Avoidance as Predictors}

I predicted that attachment anxiety and avoidance would be related to indices of intrapersonal and relationship functioning. In particular, I predicted attachment anxiety to be related to reports that one's partner's military experiences such as deployments and postings has had a more negative impact on the quality of their relationships, that various military experiences will be perceived as significantly more stressful relative to those lower in attachment anxiety. This would be consistent with the findings of Fraley and Shaver (1998) that women higher in attachment anxiety reported increased distress with long term separations. Further, consistent with findings by Collins and Read (1990), I predicted that attachment anxiety, in particular, would be related to more negative relationship perceptions. In line with findings by Mayseless and colleagues (1996) I also predicted that attachment anxiety would be related to emotion-focused coping strategies such as self-blame, and decreased coping outcomes. On the other hand, I predicted that attachment avoidance would be related to deactivating and distancing coping strategies (e.g., Mayseless et al., 1996), and would not as strongly be related to reports of well-being.

To test these predictions, I conducted multiple linear regressions with the mean centered attachment anxiety, avoidance, and interaction terms as predictors on all 30 dependent measures (listed in Table 6). Again, prior to conducting these analyses, I tested for the presence of reported confounds for attachment anxiety including self-esteem (e.g., Collins \& Read, 1990; Bartholomew \& Horowitz, 1991) relationship length (e.g., Duemmler \& Kobak, 2001; Fraley \& Shaver, 1998), whether or not the spouse had children (e.g., Medway, Davis, Cafferty, Chappell, 
\& O'Hearn, 1995), and awareness of military and/or local civilian community resources (e.g., Nurius, \& Lester, 2013). Unfortunately, an established measure of self-esteem was not included in this survey. However, one of the K10 (Kessler et al., 2010) items captures the assessment of one's worth, a defining characteristic of self-esteem (Baumeister, 1998; Rosenberg, 1965). For this reason, this item was used as a proxy measure of self-esteem. I conducted bivariate correlations between attachment anxiety and all potential covariates. There were significant relationships between attachment anxiety and perceptions of self-worth $[r(1737)=-.39, p<$ $.001]$, and awareness of military and/or local civilian community resources $[r(1734)=-.10, p<$ .001]. In addition, there was a significant relationship between avoidance and relationship length $[r(1732)=.06, p=.008]$, and awareness of support programs $[r(1735)=-.10, p<.001]$. As a result, self-worth, awareness of support programs, and relationship length were included as covariates in all analyses. Further, given that I was conducting regression analyses on 30 dependent measures and with three predictors, I decided to use the more stringent alpha criterion of $p<.001(.05 / 90)$. Therefore, about .09 results would be expected by chance alone.

\section{Main Effects of Attachment Anxiety}

There were a number of significant main effects of attachment anxiety (see Table 7 for all results). Specifically, attachment anxiety was associated with greater reported stress during general military experiences $[B=.15, \beta=.20, t(1578)=6.13, p<.001]$, decreased ability to handle daily demands $[B=-.13, \beta=-.20, t(1712)=-6.93 p<.001]$, increased desire to eat when stressed $[B=.11, \beta=.11, t(1707)=3.24, p=.001]$, decreased subjective well-being $[B=-.13, \beta$ $=-.10, t(1713)=-3.29, p<.001]$, greater psychological distress $[B=.954, \beta=.16 t(1713)=$ $6.86, p<.001]$, less mastery over one's life $[B=-.12, \beta=-.24, t(1712)=-8.33, p<.001]$, greater use of self-blame as a coping strategy $[B=.19, \beta=.26, t(1704)=9.04, p<.001]$, greater use of 
distraction as a coping strategy $[B=.08, \beta=.11, t(1705)=3.48, p=.001]$, less perceived relationship support $[B=-.19, \beta=-.22, t(1705)=-8.45, p<.001]$, less emotional fitness $[B=-$ $.13, \beta=-.15, t(1706)=-5.42, p<.001]$, less cognitive fitness $[B=-.09, \beta=-.14, t(1705)=-4.50$, $p<.001]$, less life readiness $[B=-.09, \beta=-.12, t(1710)=-3.97, p<.001]$, less coping success $[B$ $=-.16, \beta=-.20, t(1709)=-6.92, p<.001]$, less perceived relationship success $[B=-.07, \beta=-$ $.09, t(1710)=-3.39, p=.001]$, less relationship satisfaction according to two indices [QMI: $B=-$ $.26, \beta=-.23, t(1712)=-9.08, p<.001$; RDAS: $B=-.15, \beta=-.25, t(1710)=-9.17, p<.001]$, less perceived relationship consensus $[B=-.12, \beta=-.20, t(1707)=-7.40, p<.001]$, and less perceived relationship cohesion $[B=-.07, \beta=-.10 t(1711)=-3.58, p<.001]$.

\section{Main Effects of Attachment Avoidance}

There also were a number of main effects for attachment avoidance. Attachment avoidance was related to decreased subjective well-being $[B=-.30, \beta=-.21, t(1713)=-7.18, p<$ $.001], p<.001]$, less perceived social support $[B=-.10, \beta=-.09, t(1705)=-3.58, p<.001]$, greater use of behavioural disengagement as a coping strategy $[B=.09, \beta=.12, t(1703)=3.86$, $p<.001)$, less reliance on religion to cope $[B=-.10, \beta=-.11, t(1689)=-3.21, p=.001]$, less perceived relationship support $[B=-.33, \beta=-.34, t(1705)=-12.98, p<.001]$, less emotional fitness $[B=-.18, \beta=-.18, t(1706)=-6.76, p<.001]$, less cognitive fitness $[B=-.11, \beta=-.14$, $t(1705)=-4.61, p<.001]$, less social fitness $[B=-.23, \beta=-.18, t(1704)=-6.07, p<.001]$, less ethical fitness $[B=-.21, \beta=-.17, t(1544)=-5.09, p<.001]$, less perceived partner support $[B=-$ $.14, \beta=-.18, t(1708)=-5.69, p<.001]$, less coping success $[B=-.08, \beta=-.09, t(1709)=-3.20$, $p=.001]$, less perceived relationship success $[B=-.40, \beta=-.45, t(1710)=-16.75, p<.001]$, less relationship satisfaction according to both indices (QMI: $B=-.54, \beta=-.41, t(1712)=-16.19, p<$ $.001 ;$ RDAS: $B=-.23, \beta=-.32, t(1710)=-11.54, p<.001]$, less perceived relationship 
consensus $[B=-.27, \beta=-.39, t(1707)=-14.52, p<.001]$, and less perceived relationship cohesion $[B=-.34, \beta=-.41, t(1711)=-14.43, p<.001]$.

Table 7

Standardized Regression Coefficients for the Unique and Interactive Effects of Attachment Anxiety and Avoidance on all Dependent Measures.

\begin{tabular}{|c|c|c|c|c|c|c|}
\hline \multirow[b]{2}{*}{ Measure } & \multicolumn{2}{|c|}{ Anxiety } & \multicolumn{2}{|c|}{ Avoidance } & \multicolumn{2}{|c|}{ Anx*Avo } \\
\hline & $\beta$ & $t$ & $\beta$ & $t$ & $\beta$ & $t$ \\
\hline Military life impacting career & -.05 & -1.51 & .01 & 0.32 & .00 & -0.02 \\
\hline Stress during general military & .20 & $6.13^{*}$ & -.04 & -1.33 & -.00 & -0.13 \\
\hline $\begin{array}{l}\text { Stress during partner injury or } \\
\text { combat deplopyment }\end{array}$ & .07 & 1.62 & .02 & 0.34 & -.01 & -0.29 \\
\hline Ability to handle daily demands & -.18 & $-5.81^{*}$ & -.08 & -2.49 & .07 & $2.76^{+}$ \\
\hline Desire to eat when stressed & .11 & $3.29^{*}$ & -.03 & -0.86 & -.02 & -0.66 \\
\hline Subjective well-being & -.10 & $-3.43^{*}$ & -.21 & $-7.18^{*}$ & -.01 & -0.33 \\
\hline Psychological distress & .16 & $6.86^{*}$ & .04 & 1.57 & -.04 & -2.40 \\
\hline Mastery & -.23 & $-8.33^{*}$ & -.08 & $-2.79^{*}$ & .05 & 2.37 \\
\hline Active coping factor & .03 & 1.02 & -.09 & $-2.78^{+}$ & -.07 & $-2.68^{+}$ \\
\hline Social support coping factor & .07 & 2.28 & -.12 & $-3.58^{*}$ & -.10 & $-3.67^{*}$ \\
\hline Self-blame coping & .26 & $9.07^{*}$ & -.02 & -0.79 & -.10 & $-4.56^{*}$ \\
\hline Denial coping & .08 & $2.57^{+}$ & .04 & 1.25 & -.00 & -0.18 \\
\hline Substance use coping & .10 & $2.98^{+}$ & .04 & 1.15 & -.01 & -0.40 \\
\hline $\begin{array}{l}\text { Behavioural disengagement } \\
\text { coping }\end{array}$ & .03 & 1.04 & .12 & $3.88^{*}$ & .01 & 0.54 \\
\hline Self-distraction coping & .11 & $3.48^{*}$ & -.02 & -0.53 & -.08 & $-3.28^{*}$ \\
\hline Religion coping & .03 & 0.82 & -.11 & $-3.21^{*}$ & -.03 & -1.08 \\
\hline Relationship support & -.22 & $-8.45^{*}$ & -.34 & $-12.98^{*}$ & -.15 & $-7.37^{*}$ \\
\hline Physical fitness & -.09 & $-2.75^{+}$ & -.09 & $-2.87^{+}$ & .00 & 0.07 \\
\hline Emotional fitness & -.15 & $-5.42^{*}$ & -.18 & $-6.80^{*}$ & .02 & 0.95 \\
\hline Cognitive fitness & -.14 & $-4.50^{*}$ & -.14 & $-4.61^{*}$ & .02 & 0.93 \\
\hline Social fitness & .02 & 0.50 & -.18 & $-6.07^{*}$ & .02 & 0.74 \\
\hline Ethical fitness & .01 & 0.31 & -.17 & $-5.14^{*}$ & .09 & $3.55^{*}$ \\
\hline Life readiness & -.12 & $-3.97^{*}$ & -.05 & -1.61 & .07 & 3.04 \\
\hline Partner support & -.02 & -0.48 & -.18 & $-5.69^{*}$ & .06 & $2.61^{+}$ \\
\hline Coping success & -.20 & $-6.92^{*}$ & -.09 & $-3.20^{*}$ & .05 & 2.21 \\
\hline Relationship success & -.09 & $-3.39^{*}$ & -.45 & $-16.75^{*}$ & -.10 & $-4.54^{*}$ \\
\hline Relationship satisfaction & -.23 & $-9.08^{*}$ & -.41 & $-16.19^{*}$ & -.08 & $-4.09^{*}$ \\
\hline Dyadic consensus & -.20 & $-7.40^{*}$ & -.39 & $-14.52^{*}$ & -.02 & -1.14 \\
\hline Dyadic satisfaction & -.25 & $-9.17^{*}$ & -.31 & $-11.54^{*}$ & -.05 & -2.36 \\
\hline Dyadic cohesion & -.10 & $-3.58^{*}$ & $-.41^{*}$ & $-14.43^{*}$ & -.02 & -0.96 \\
\hline
\end{tabular}


Two-Way Interactions Between Attachment Anxiety and Attachment Avoidance

These main effects were qualified by a number of anxiety-by-avoidance interactions. All two-way interactions were decomposed using simple slopes analyses (Aiken \& West, 1991), examining the effects of attachment anxiety at lower (-1 SD from the mean) and higher (+1 SD from the mean) levels of attachment avoidance.

There was a significant two-way interaction for use of social support as a coping strategy, $[B=-.06, \beta=-.10, t(1705)=-3.67, p<.001]$. At lower levels of attachment avoidance, there was a significant positive relationship between attachment anxiety and the use of social support as a coping strategy $[B=.11, \beta=.16, t(1705)=3.82, p<.001$; see Figure 26]. However, at higher levels of attachment avoidance, the relationship between attachment anxiety and the use of social support was not significant $[B=-.01, \beta=-.01, t(1705)=-0.21, p=.833)$.

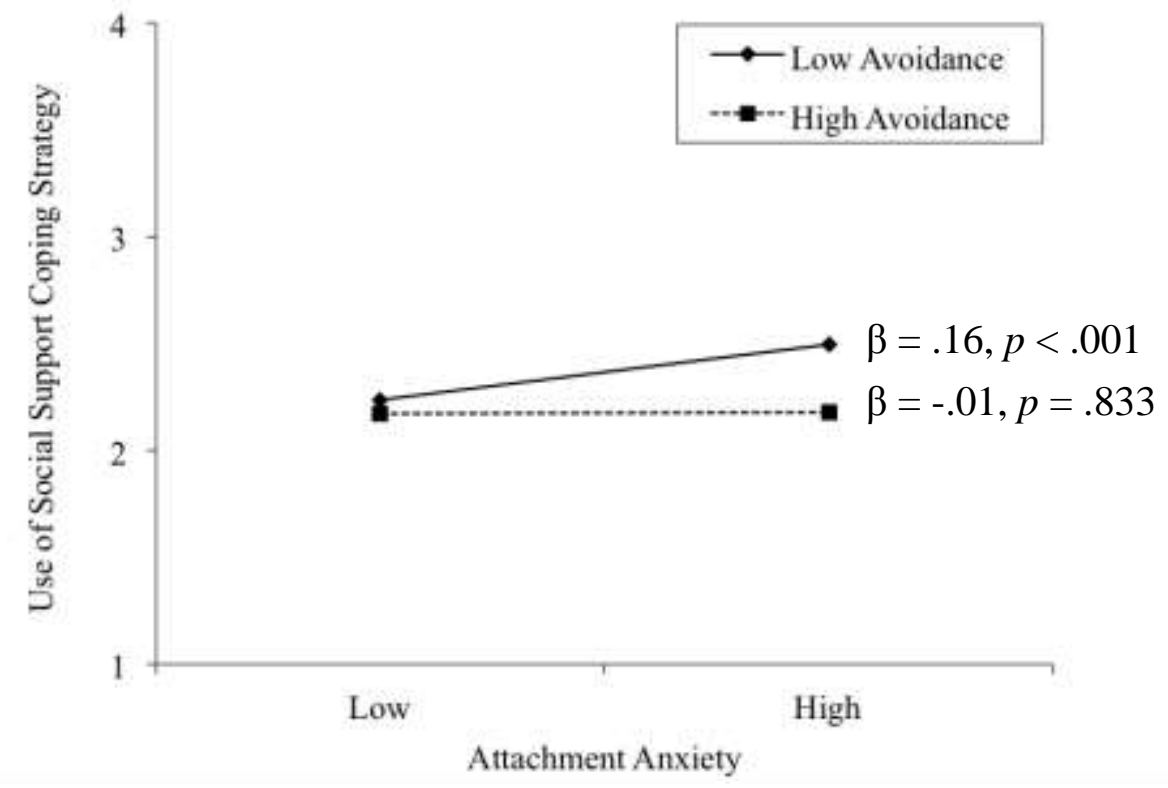

Figure 26. Interaction between attachment anxiety and attachment avoidance on the use of social support as a coping strategy. 
Next, there was a significant two-way interaction for use of self-blame as a coping strategy, $[B=-.07, \beta=-.10, t(1704)=-4.56, p<.001]$. At lower levels of attachment avoidance, there was a significant positive relationship between attachment anxiety and the use of selfblame $[B=.26, \beta=.35, t(1704)=9.69, p<.001$; see Figure 27). Further, at higher levels of attachment avoidance, there was a significant positive relationship, albeit weaker than the previous, between attachment anxiety and the use of self-blame $[B=.13, \beta=.17, t(1704)=5.11$, $p<.001]$.

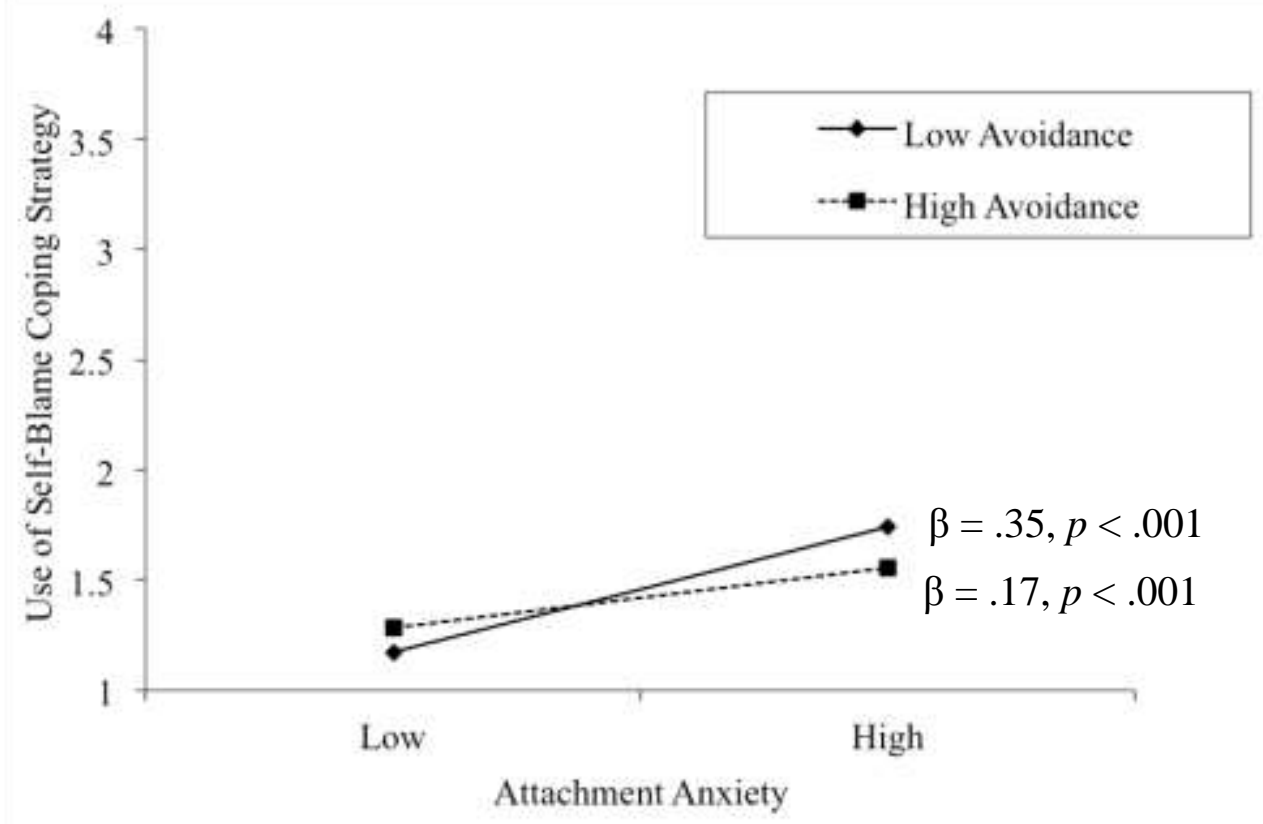

Figure 27. Interaction between attachment anxiety and attachment avoidance on the use of selfblame as a coping strategy.

There was a significant two-way interaction for use of self-distraction as a coping strategy, $[B=-.06, \beta=-.08, t(1705)=-3.28, p=.001]$. At lower levels of attachment avoidance, there was a significant positive relationship between attachment anxiety and the use of selfdistraction as a coping strategy $[B=.14, \beta=.19, t(1705)=4.53, p<.001$; see Figure 28]. 
However, at higher levels of attachment avoidance, the relationship between attachment anxiety and the use of self-distraction was not significant $[B=.03, \beta=.04, t(1705)=1.04, p=.296)$.

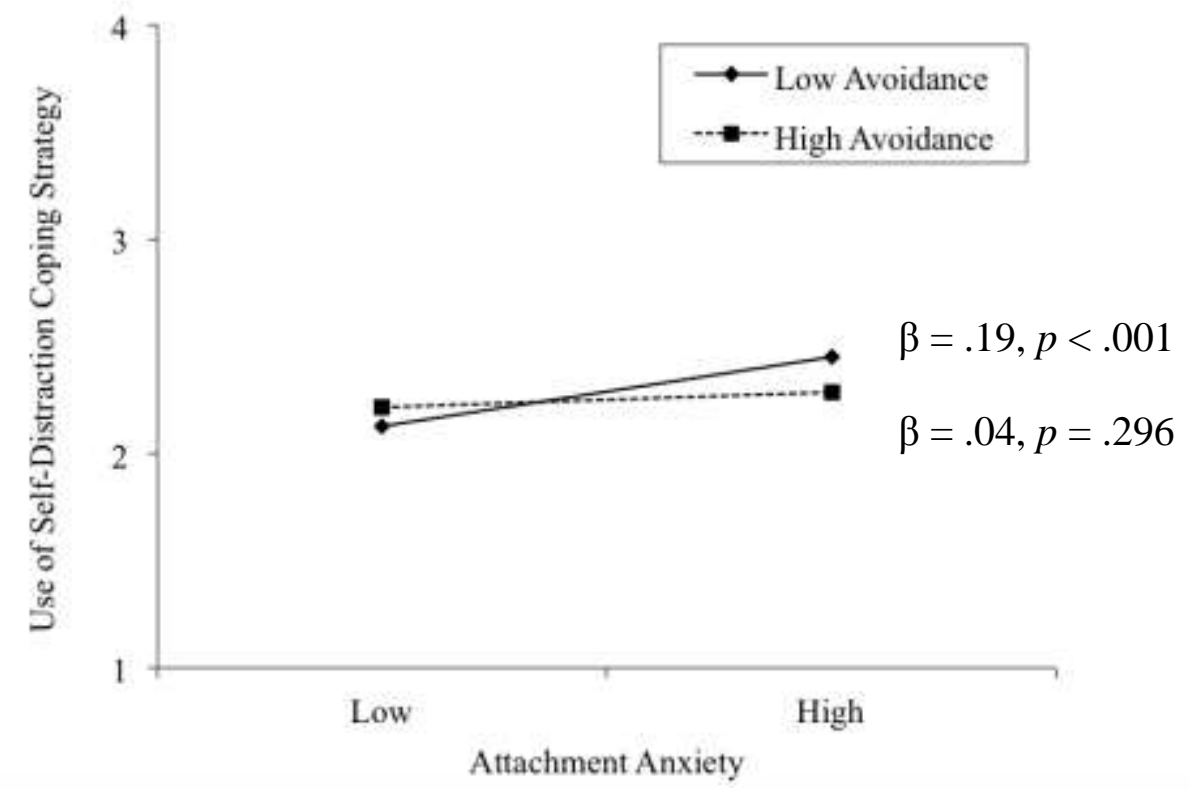

Figure 28. Interaction between attachment anxiety and attachment avoidance on the use of selfdistraction as a coping strategy.

Next, there was a significant two-way interaction for perceived relationship support $[B=$ $-.11, \beta=-.15, t(1705)=-7.37, p<.001]$. At lower levels of attachment avoidance, the relationship between attachment anxiety and relationship support approached significance $[B=-$ $.08, \beta=-.09, t(1705)=-2.69, p=.007$; see Figure 29]. At higher levels of attachment avoidance, there was a significant negative relationship between attachment anxiety and relationship support $[B=-.30, \beta=-.36, t(1705)=-11.58, p<.001]$.

Next, there was a two-way interaction for perceived relationship success $[B=-.06, \beta=-$ $.09, t(1710)=-4.54, p<.001]$. At lower levels of attachment avoidance, the relationship between attachment anxiety and relationship success was not significant $[B=-.01, \beta=-.01$, $t(1710)=-0.22, p=.823$; see Figure 30]. However, at higher levels of attachment avoidance 
there was a significant negative relationship between anxiety and relationship success $[B=-.13$, $\beta=-.18, t(1710)=-5.57, p<.001]$

Finally, there was also a two-way interaction for the QMI measure of relationship satisfaction $[B=-.08, \beta=-.08, t(1712)=-4.09, p<.001]$. At lower levels of attachment avoidance, there was a significant negative relationship between attachment anxiety and relationship satisfaction $[B=-.18, \beta=-.16, t(1712)=-4.98, p<.001$; see Figure 31$)$. In addition, at higher levels of attachment avoidance there was a significant negative relationship (albeit stronger) between anxiety and relationship satisfaction significant $[B=-.35, \beta=-.30, t(1712)=-$ $10.19, p<.001]$.

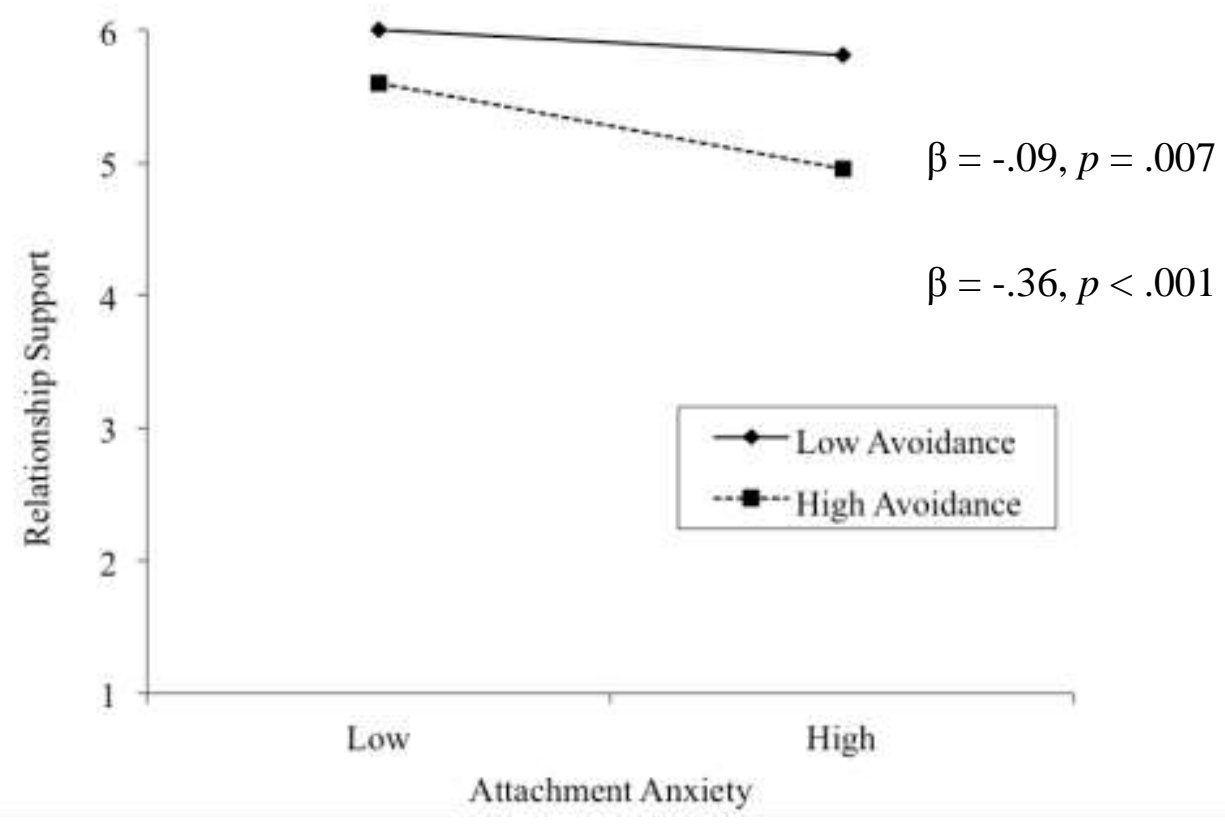

Figure 29. Interaction between attachment anxiety and attachment avoidance on perceived relationship support. 


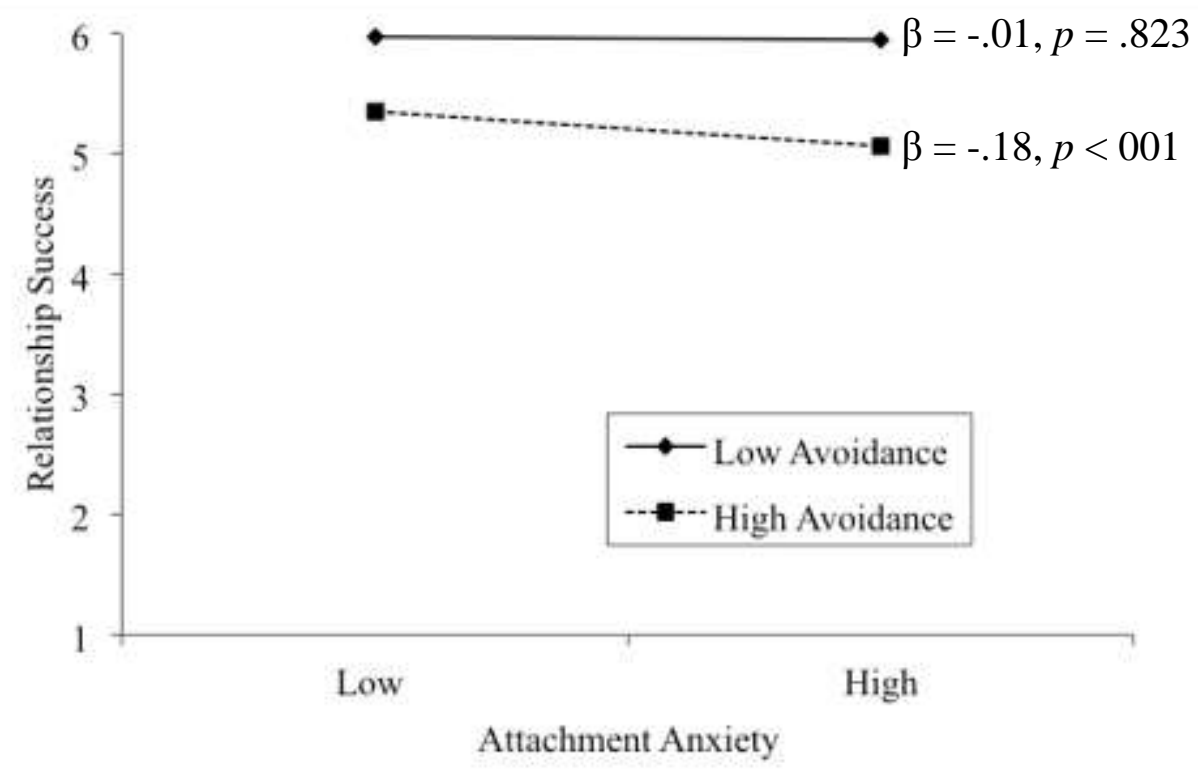

Figure 30. Interaction between attachment anxiety and attachment avoidance on relationship success.

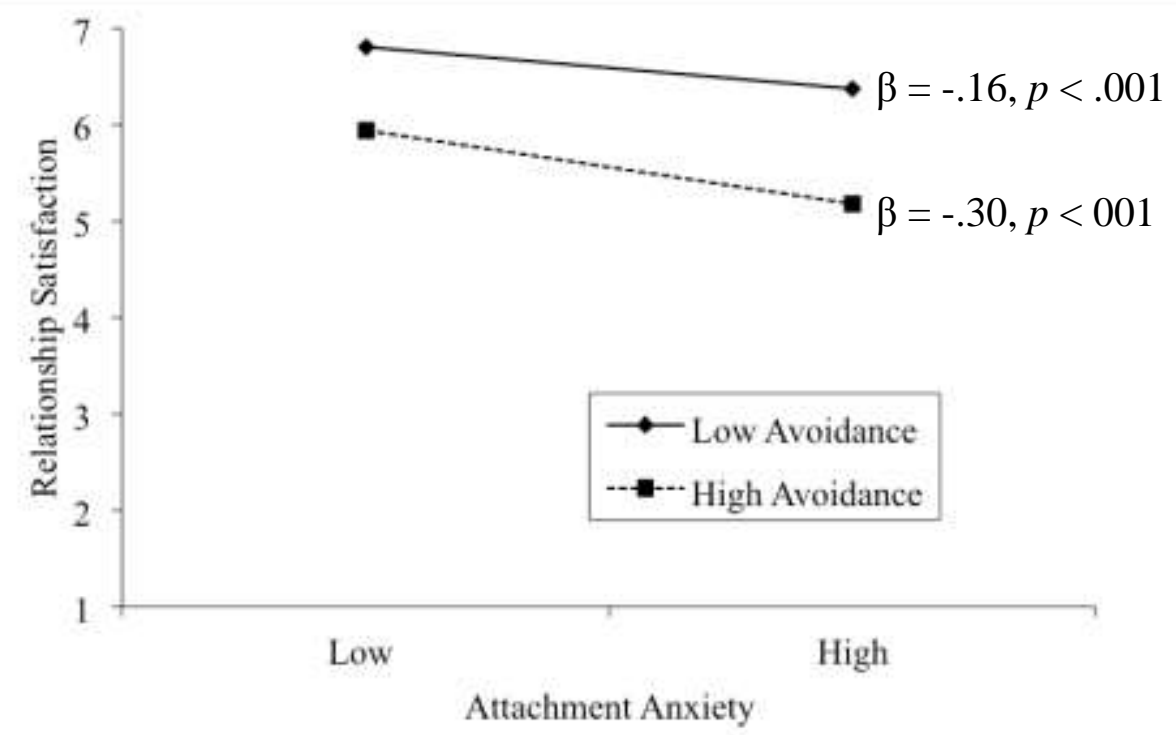

Figure 31. Interaction between attachment anxiety and attachment avoidance on relationship satisfaction (QMI). 


\section{Attachment Anxiety, Avoidance, and Deployment Status}

Although main effects of attachment dimensions are often reported and were therefore expected, individual differences in attachment behaviour should be most pronounced under stressful conditions in the social or physical environment (Simpson \& Rholes, 1994). Therefore, I predicted that the effects of attachment dimensions on intrapersonal coping and relationship outcomes might be moderated by deployment status, or whether or not the spouses were currently separated by their partners from a military deployment. Specifically, attachment anxiety is associated with hyperactivating strategies in response including increased distress and emotion-focused coping strategies in response to attachment threats (Birnbaum et al., 1997; Fraley \& Shaver, 1998; Mayseless et al., 1996, Meyer et al., 2005). However, attachment avoidance should be associated with deactivating strategies aimed at promoting interpersonal distance when the attachment system is activated (Fraley \& Shaver, 1997; Fraley \& Shaver, 1998; Mayseless et al., 1996; Simpson et al., 1992). Therefore, if deployment status entails different levels of threat, attachment anxiety should be predictive of decreased well-being, the use of emotion-focused coping strategies, and potentially more negative relationship perceptions for those separated from their partners, relative to those that are not. On the other hand, attachment avoidance might be less predictive of, or even associated with increased emotional well-being, but perhaps more negative relationship perceptions for those experiencing a deployment, relative to those not experiencing a deployment.

Specifically, I was interested in assessing how deployment status might moderate the relationships between attachment anxiety, attachment avoidance, their interaction, and 18 of the associated outcomes reported above. A total of 211 spouses had partners who were currently deployed, while 1,301 had spouses who were not currently deployed, not preparing for a 
deployment, and had not returned from a deployment in the last 12 months. When making comparisons between samples or using grouping variables as moderators, large discrepancies in sample sizes can compromise the validity of null hypothesis decisions. Specifically, differences in sample sizes can lead to heterogeneity of variance, which in the case of regression analyses may ultimately threaten the assumption of homoscedasticity if error variances are unequal across levels of a moderator, and also potentially unequal across combinations of the IV and moderating variables. In order to avoid such issues, I decided to use a random sample of 211 spouses with non-deployed partners (an $n$ equal to that of the group of spouses whose partners were deployed), whose spouses had not recently (last 12 months) experienced a deployment, and who were not currently preparing for a deployment. After selecting my random sub sample of 211 spouses whose partners were not currently deployed, I decided to conduct t-tests (or chisquare tests where appropriate) between my two groups to ensure that they did not differ in important ways (demographics and attachment dimensions) other than the key dependent variables and therefore to identify any potential control variables.

The two groups were not statistically different with respect to being married to a same or opposite sex partner, gender, age, education, whether the spouse has children, the spouse's employment status, whether they were aware of support programs within their community, their partner's rank and element, and levels of attachment avoidance, or anxiety ${ }^{19}$ ( $p$ s between .24.85). However, deployed partners had experienced significantly more deployments in the past five years $(M=2.50, S D=2.79)$ relative to the non-deployed partners $(M=1.03, S D=2.04)$, $t(374)=-6.1, p<.001)$. Further, the proportion of spouses who were currently CAF members

\footnotetext{
${ }^{19}$ I also conducted t-tests or chi-square tests between my random sample of non-deployed partners and the rest of the group on these same variables to ensure that my sample was representative. The random sample was not statistically different from the rest of the group on any of these variables, suggesting it was indeed representative.
} 
(regular or reserve force) or civilians was not consistent across the two groups, $\chi^{2}(3, N=416)=$ $9.80, p=.020$. Specifically, the deployed group had a greater proportion of at-home spouses who were regular force members than expected, and the non-deployed group has fewer regular force spouses than expected. Further, relationship status was not consistent across the two groups, $\chi^{2}(5, N=418)=14.14, p=.15$. Specifically, the deployed group had a greater proportion of common-law relationships than expected, and the non-deployed group had a greater proportion of legally married relationships than expected. For this reason, number of deployments experienced in the past five years, spouse CAF status, and relationship status were included as covariates in subsequent analyses. As in the previous analyses, a measure of selfworth, relationship length, and awareness of support programs were also included as covariates. Deployment status was coded such that spouses of non-deployed members were assigned a value of -1 , and spouses of deployed members were assigned a value of 1 .

Given that I had already assessed the effects of attachment anxiety, avoidance, and their interaction term on the following indices, I am only reporting below the moderating effects of partner deployment status on the attachment dimensions. Specifically, in addition to reporting main effects of deployment status, I am reporting whether there were significant two-way interactions between deployment status and avoidance, deployment status and anxiety, or a three-way interaction among deployment status, avoidance, and anxiety. Many of these analyses were exploratory in nature, given the sparse amount of research assessing the moderating effects of deployment-related experiences in the relationships among attachment dimensions and intrapersonal and relationship outcomes. For this reason, I was hesitant to employ a conservative Bonferroni correction like in previous analyses and risk inflating Type II error rates. However, there is a trade off in not employing any correction at all, leading to inflations in Type I errors. 
However, I had fairly clear predictions about the nature of these interactions (that the effects of anxiety would be exacerbated for spouses whose partners were currently deployed), and the measures with which I would find these effects (those same key measures that effects of anxiety were reported for). All of the above considered, I decided to employ a more conservative probability criterion of .01 , which is one of the more frequently used conservative levels of significance (e.g., Hinkle, Wiersma, \& Jurs, 2003; Tabachnick \& Fidell, 2007). Therefore, the following results were significant if they met the corrected level of significance, $p<.01$. It is important to note that I was conducting 54 analyses (18 dependent measures and three predictors), which would lead to .5 findings being produced by chance with an alpha criterion of .01. Here, two findings were statistically significant.

First, there were no significant two-way interactions between anxiety and deployment status. There were, however, significant two-way interactions between avoidance and deployment status. I decomposed these interactions using simple slopes analysis (Aiken \& West, 1991) examining the effects of deployment status at low (-1 SD) and high (+1 SD) levels of attachment avoidance. Specifically, there was a significant two-way relationship between avoidance and deployment status on perceived relationship support, $B=-.15, \beta=-.16, t(390)=$ $2.85, p=.005$. At high levels of attachment avoidance, there was a significant negative relationship between deployment status and perceived relationship support, $B=-.21, \beta=-.23$, $t(391)=-2.85 p=.005$ (see Figure 32). However, at low levels of attachment avoidance, the relationship between deployment status and perceived relationship support was not significant, $B$ $=.07, \beta=.08, t(391)=1.23 p=.221$. 


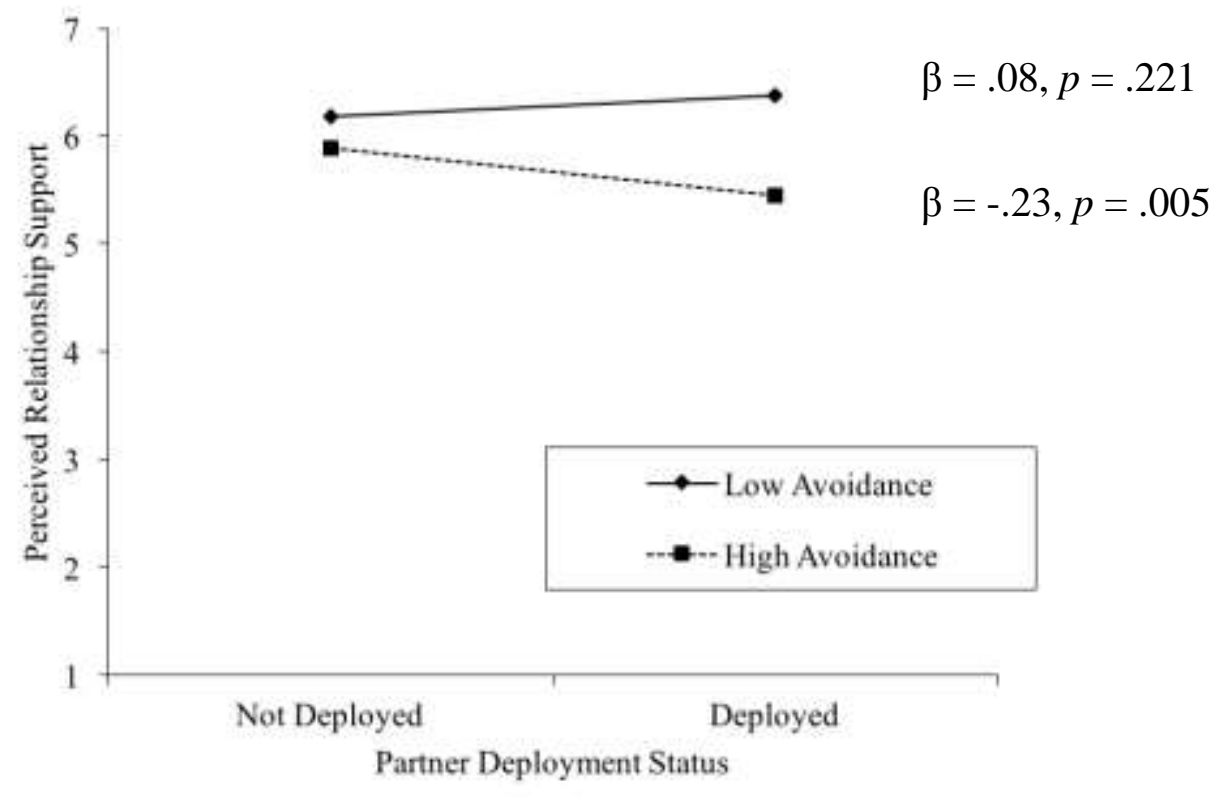

Figure 32. Interaction between attachment avoidance and deployment status on perceived relationship support.

In addition, there was a two-way relationship between avoidance and deployment status on cognitive fitness, $B=-.13, \beta=-.17, t(390)=-2.66, p=.008$. At high levels of avoidance, there was a significant negative relationship between deployment status and cognitive fitness, $B$ $=-.16, \beta=-.22, t(391)=-2.33, p=.020$ (see Figure 33). However, at low levels of avoidance, the relationship between deployment status and cognitive fitness was not significant, $B=.10, \beta=$ $.14, t(391)=1.82, p=.069$. 


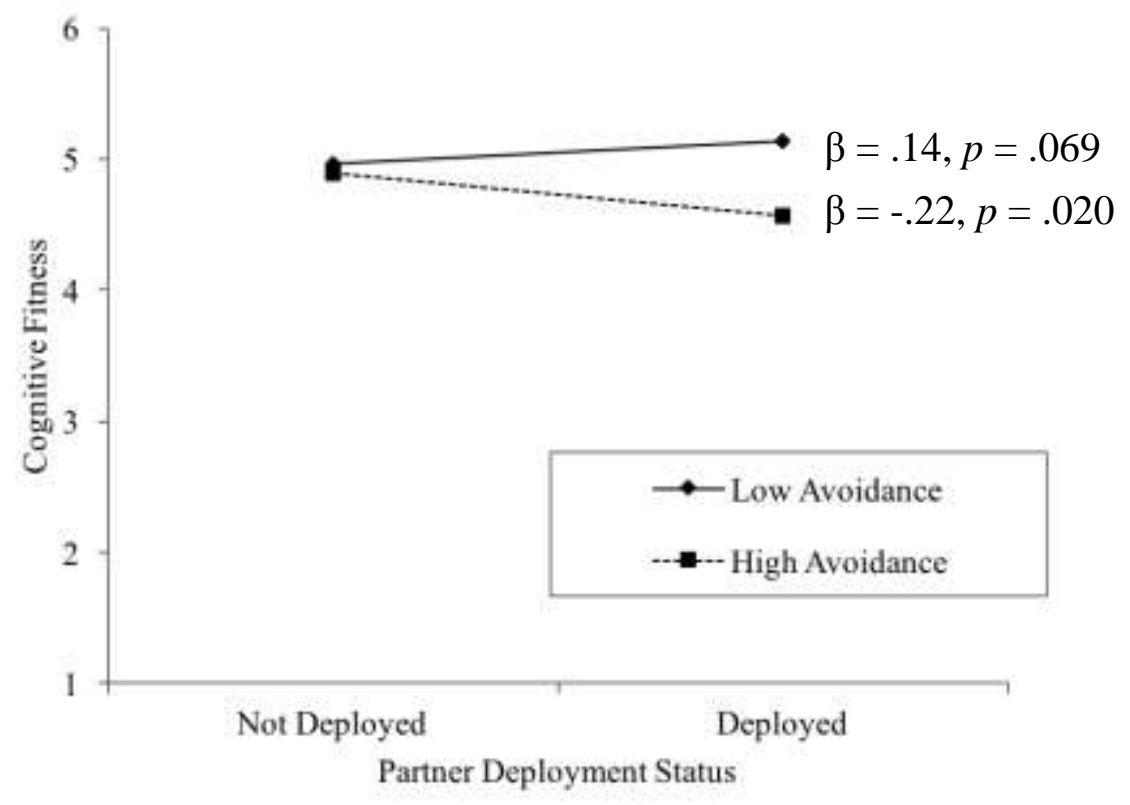

Figure 33. Interaction between attachment avoidance and deployment status on cognitive fitness.

There were no significant two-way interactions between attachment anxiety and deployment status, nor were there significant three-way interactions among attachment anxiety, avoidance, and deployment status

\section{Attachment Anxiety, Avoidance, and Recent Deployment Status}

In summary, the above findings largely did not confirm my predictions, that the effects of attachment anxiety (and avoidance) on intrapersonal and relationship functioning would be exacerbated for the spouses whose partners are currently away on a military deployment.

Instead, although some effects for the interaction between anxiety and avoidance were reported, overall deployment status did not seem to moderate the effects of attachment anxiety on most intrapersonal functioning and relationship outcomes. I wondered if these results were in part due to the fact that the spouses of deployed members had been separated from their partners for an average of 6.62 months ( $S D=9.91$ months). Specifically, there is good reason to expect that the effects of attachment dimensions should be most pronounced under conditions that threaten the 
attachment relationship (Feeney, 1999; Simpson \& Rholes, 1994). In particular, attachment anxiety should be associated with hyperactivating strategies to cope with the recent separation such as increased reports of distress (Fraley \& Shaver, 1998; Mayseless et al., 1996), and perhaps even decreased relationship perceptions (Rholes et al., 2001). However, attachment avoidance should be associated with deactivating strategies to manage the threat of being separated from one's partners such as pulling away from the partner emotionally and reporting more negative relationship perceptions and refraining from managing the threat such as reporting less issues with coping capacity (Mayseless et al., 1996; Simpson et al., 1992). There is the possibility, however, that breakdowns in emotional well-being and coping capacity will be observed with increases in attachment avoidance, if this initial separation period is characterized by high enough levels of stress that deactivating strategies are less effective (Mikulincer \& Florian, 1998).

As outlined in the Canadian Forces Deployment Handbook (Egerton-Graham, 2009), the most challenging stage of the separation is stage 3, occurring during weeks 3-6 of the separation, and consisting of emotional disorganization. Our questionnaire did not assess time separated in weeks, therefore I decided to examine the group of spouses who had most recently ( $\leq 1$ month) separated from their partners $(n=64)$ and who were likely experiencing emotional disorganization, and see whether these spouses demonstrate more pronounced attachment effects than those not currently experiencing a partner separation. In order to compare this group of spouses with an equal number of spouses with non-deployed partners, I again selected a random sample of 64 spouses of non-deployed partners, with the conditions that they also could not have returned from a deployment in the last 12 months, and were not currently preparing for a deployment ( $n=1293)$. I also conducted t-tests (or chi-squared tests where appropriate) on the 
same key demographic items and attachment dimensions that I tested between my samples earlier, but now between both my random sub-sample of non-deployed spouses against the rest of the non-deployed group, and between my non-deployed and deployed partner groups. There were no significant differences between the non-deployed sub-sample and rest of the nondeployed sample and recently deployed samples, suggesting this sub-sample was representative of the main sample of spouses of non-deployed partners. However, there was a significant difference between my recently deployed and non-deployed groups such that the spouses of recently deployed partners had experienced more deployments in the past five years $(M=3.21$, $S D=4.14)$, relative to the spouses of partners not deployed $(M=1.00, S D=1.23), t(73)=-4.05$, $p<.001$. Therefore, number of deployments in the past five years was included as a covariate in subsequent analyses, along with relationship status, self-worth, relationship length, and awareness of support programs consistent with prior analyses. Specifically, I ran the same multiple regressions for the $18 \mathrm{DVs}$ that attachment anxiety significantly predicted earlier. Similar to the previous analyses, recent deployment status ( $\leq 1$ month) was coded such that spouses of partners deployed were assigned a value of 1 , and those not deployed were assigned a value of -1. Also, simple slopes analyses were used to decompose any two and three-way interactions. Similar to previous analyses, I employed an alpha correction, such that the following results were significant if they met the corrected level of significance, $p<.01$. Again, because I was conducting 54 analyses (18 dependent measures and three predictors), .5 findings would be produced by chance with an alpha criterion of .01 . Here, 9 findings were statistically significant.

There was one significant main effect of recent deployment status, such that one's ability to handle daily hassles was positively related to one's partner being recently deployed (relative 
to not deployed), $B=.21, \beta=.25, t(111)=2.40, p=.018$. In other words, spouses of recently deployed members were reporting a greater ability to handle daily hassles, relative to spouses of non-deployed members.

There were also two-way interactions between avoidance and recent deployment status. Specifically, there was a significant two-way interaction between avoidance and recent deployment status on perceived relationship support, $B=-.34, \beta=-.31, t(111)=-3.14, p=.002$ (see Figure 34). Specifically, at low levels of avoidance, there was a significant positive relationship between recent deployment status and perceived relationship support, $B=.45, \beta=$ $.41, t(111)=3.47, p=.001$. However, at high levels of avoidance the relationship between recent deployment status and perceived relationship support was not significant, $B=-.20, \beta=-$ $.19, t(111)=-1.36, p=.175$.

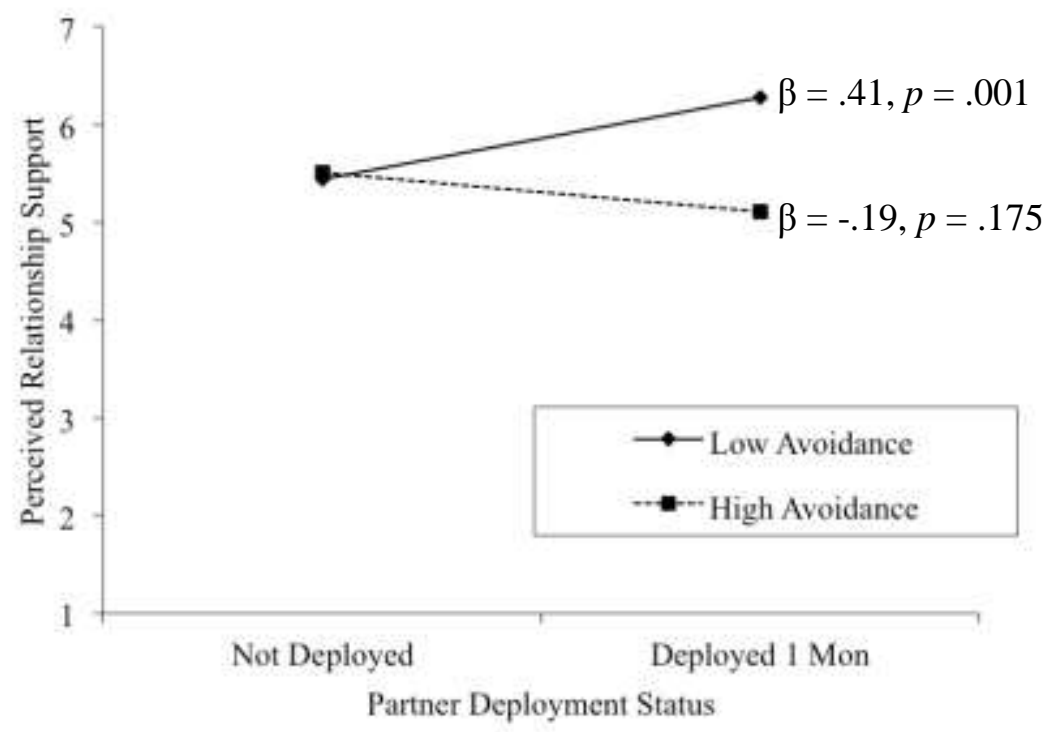

Figure 34. Interaction between attachment avoidance and recent deployment status ( $<1$ mon) on perceived relationship support. 
There was also a significant two-way interaction between attachment avoidance and recent deployment status on cognitive fitness, $B=-.31, \beta=-.36, t(111)=-3.30, p=.001$ (see Figure 35). Consistent with the above findings, at low levels of avoidance, there was a significant positive relationship between recent deployment status and cognitive fitness, $B=.29$, $\beta=.35, t(111)=2.58, p=.011$. This time, at high levels of avoidance there was a significant negative relationship between recent deployment status and cognitive fitness, $B=-.28, \beta=-.34$, $t(111)=-2.20, p=.030$.

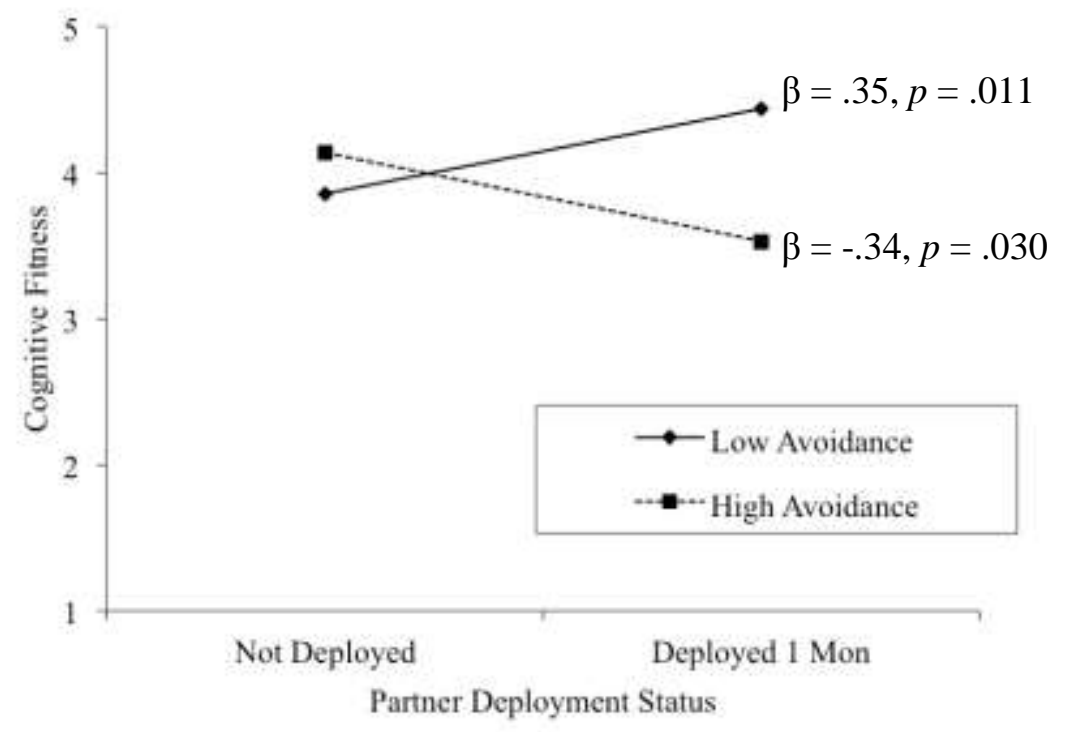

Figure 35. Interaction between attachment avoidance and recent deployment status $(<1$ mon $)$ on cognitive fitness.

In addition, there was a significant two-way interaction between avoidance and recent deployment status for perceived relationship success, $B=-.35, \beta=-.39, t(111)=-3.63, p<.001$ (see Figure 36). Consistent with the prior analyses, at low levels of avoidance, there was a significant positive relationship between recent deployment status and relationship success, $B=$ $.37, \beta=.41, t(111)=3.11, p=.002$. However, at high levels of avoidance there was a significant 
negative relationship between recent deployment status and relationship success, $B=-.31, \beta=-$ $.35, t(111)=-2.32, p=.022$.

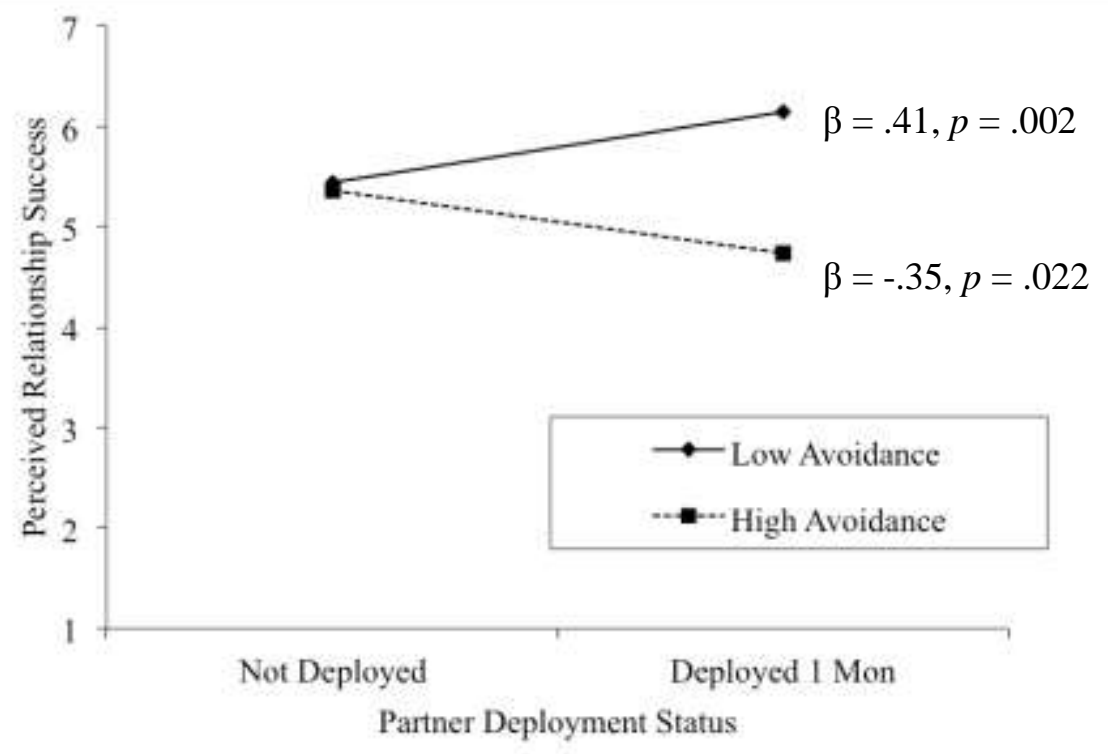

Figure 36. Interaction between attachment avoidance and recent deployment status ( $<1$ mon) on perceived relationship success.

There was also a significant two-way interaction between attachment avoidance and deployment status for relationship satisfaction using the RDAS measure, $B=-.23, \beta=-.27$, $t(111)=-2.75, p=.009$ (see Figure 37). Specifically, at low levels of attachment avoidance, there was a significant positive relationship between recent deployment status and relationship satisfaction, $B=.25, \beta=.31, t(111)=2.55, p=.012$. However, at high levels of avoidance, the relationship between recent deployment status and relationship satisfaction was not significant, $B$ $=-.21, \beta=-.25, t(111)=-1.82, p=.072$. 


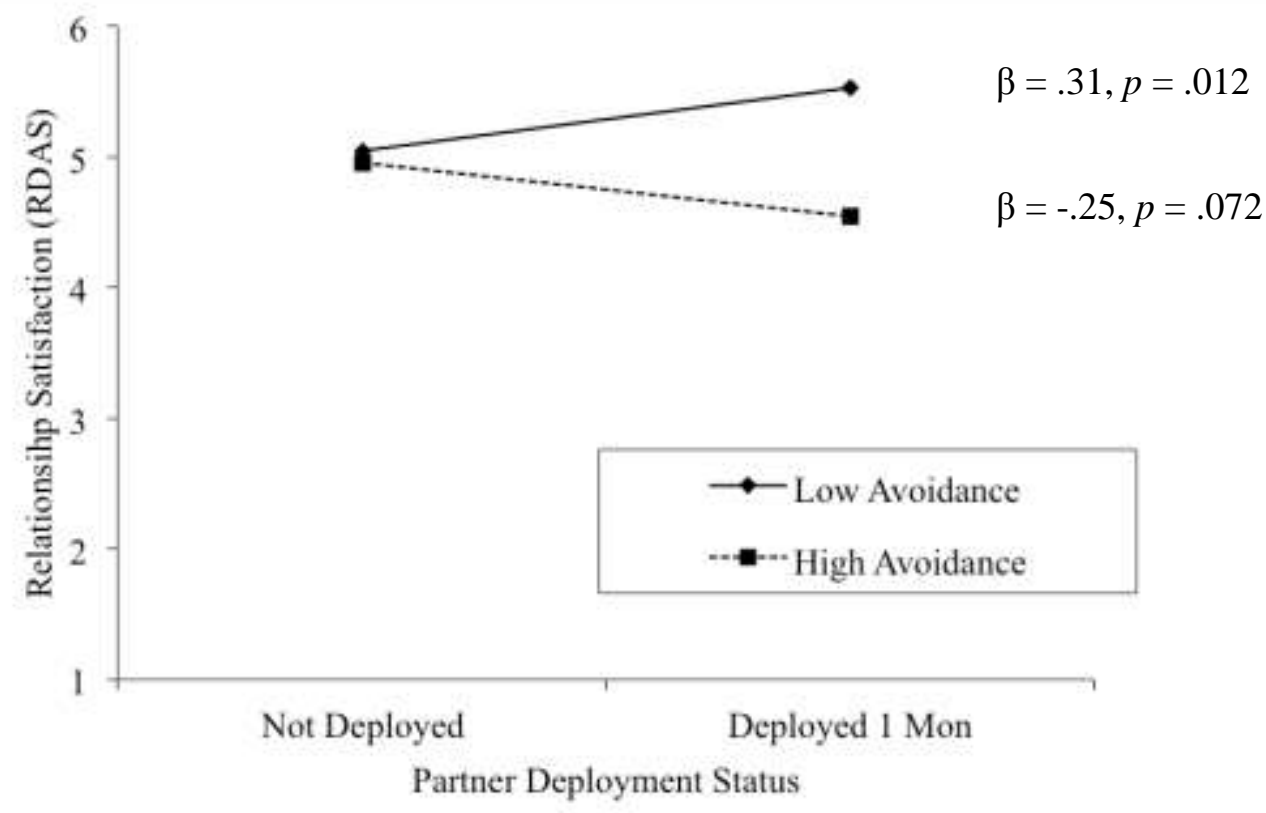

Figure 37. Interaction between attachment avoidance and recent deployment status (<1 mon) on relationship satisfaction (RDAS).

Lastly, there was a significant two-way interaction between attachment avoidance and recent deployment status for perceived relationship cohesion, $B=-.26, \beta=-.35, t(111)=-3.55, p$ $<.001$ (see Figure 38). Specifically, at low levels of attachment avoidance, there was a significant positive relationship between recent deployment status and relationship cohesion, $B=$ $.31, \beta=.43, t(111)=3.52, p=.001$. Further, at high levels of avoidance, there was a significant negative relationship between recent deployment status and relationship cohesion, $B=-.21, \beta=-$ $.29, t(111)=-2.04, p=.044$. 


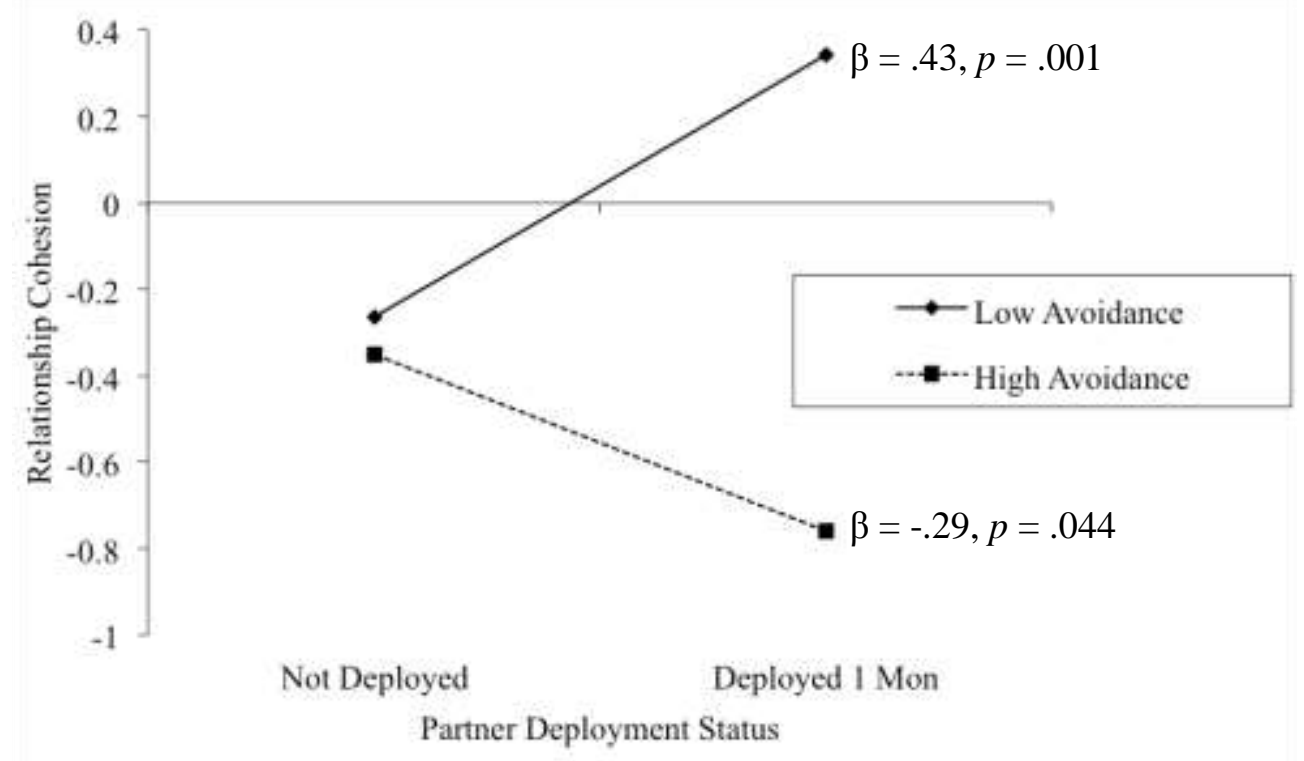

Figure 38. Interaction between attachment avoidance and recent deployment status ( $<1 \mathrm{mon})$ on relationship cohesion.

There also were significant two-way interactions between anxiety and recent deployment status. I decomposed these interactions using simple slopes analyses (Aiken \& West, 1991), examining the effects of recent deployment status at low (-1 SD) and high (+1 SD) levels of attachment anxiety. First, there was a significant two-way interaction for perceived relationship support, $B=.28, \beta=.31, t(111)=3.19, p=.002$ (see Figure 39). At low levels of anxiety, the relationship between recent deployment status and relationship support was not significant, $B=$ $.18, \beta=-.17, t(111)=-1.39, p=.165$. However, at high levels of anxiety, there was a significant positive relationship between deployment status and relationship support, $B=.44, \beta=.41, t(111)$ $=3.29, p=.001$. 


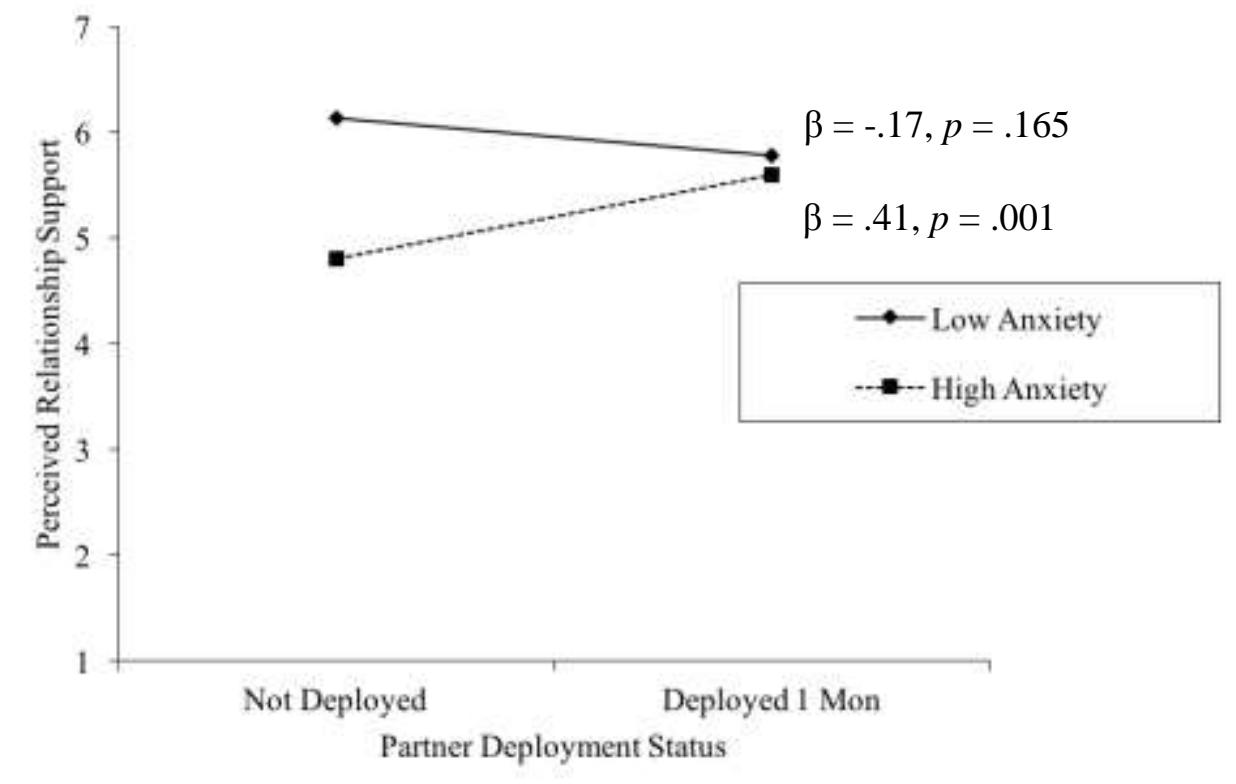

Figure 39. Interaction between attachment anxiety and recent deployment status ( $<1$ mon) on perceived relationship support.

There was also a significant two-way interaction between attachment anxiety and recent deployment status for cognitive fitness, $B=.25, \beta=.36, t(110)=3.46, p=.001$ (see Figure 40). At low levels of anxiety, there was a significant negative relationship between recent deployment status and cognitive fitness, $B=-.27, \beta=-.33, t(110)=-2.41, p=.018$. However, at high levels of anxiety, there was a significant positive relationship between deployment status and cognitive fitness, $B=.28, \beta=.34, t(110)=2.47, p=.015$.

There was a significant two-way between attachment anxiety and recent deployment status for perceived relationship success, $B=.32, \beta=.43, t(110)=4.32, p<.001$ (see Figure 41). At low levels of anxiety, there was a significant negative relationship between recent deployment status and relationship success, $B=-.33, \beta=-.38, t(110)=-2.85, p=.005$. However, at high levels of anxiety, there was a significant positive relationship between deployment status and relationship success, $B=.38, \beta=.43, t(110)=3.25, p=.002$. 


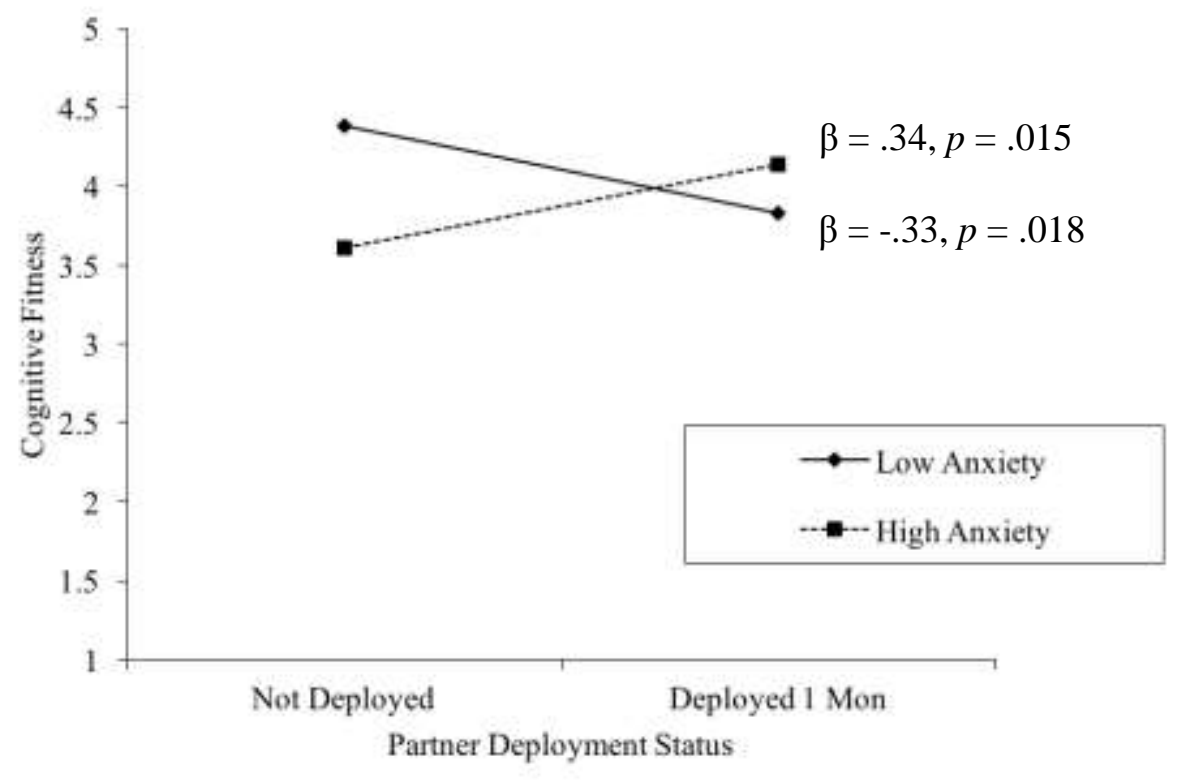

Figure 40. Interaction between attachment anxiety and recent deployment status ( $<1$ mon) on cognitive fitness.

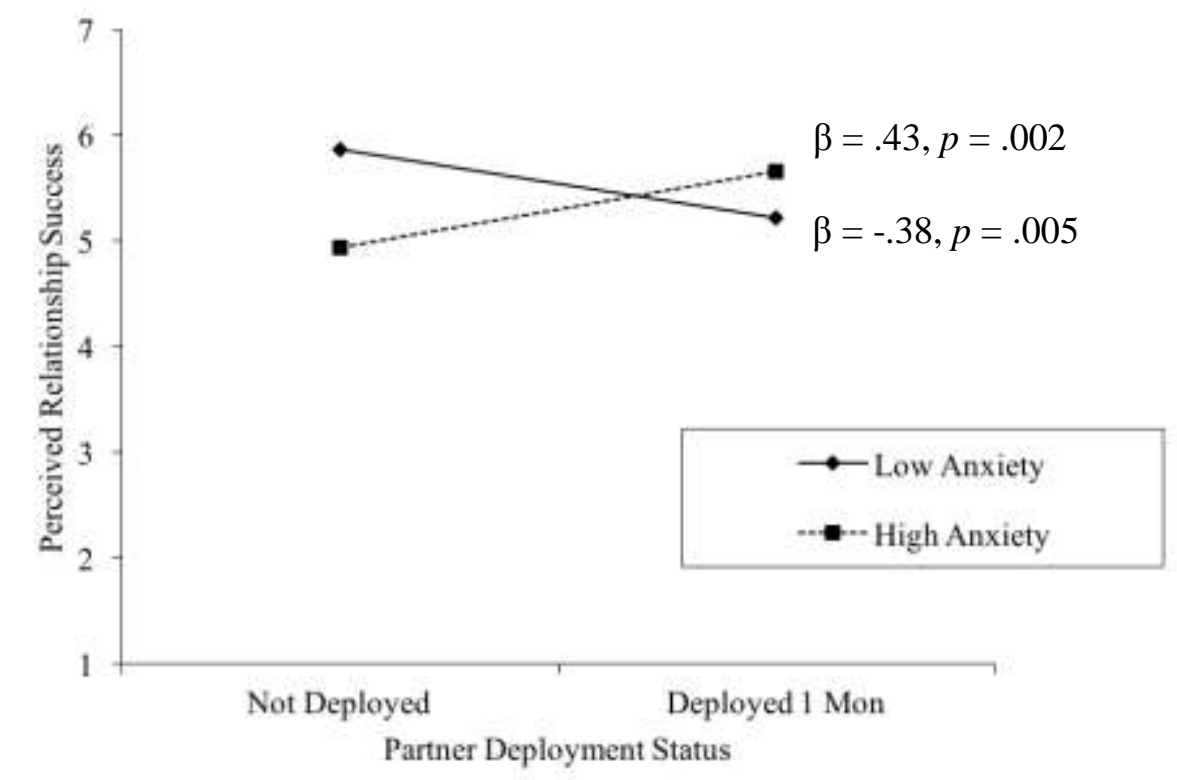

Figure 41. Interaction between attachment anxiety and recent deployment status ( $<1$ mon) on perceived relationship success. 
Finally, there was a two-way interaction for relationship satisfaction using the RDAS measure, $B=.19, \beta=.27, t(111)=2.96, p=.004$ (see Figure 42). At low levels of anxiety the relationship between recent deployment status and relationship satisfaction was not significant, $B$ $=-.19, \beta=-.23, t(111)=-1.89, p=.062$. However, at high levels of anxiety there was a significant positive relationship between recent deployment status and relationship satisfaction, $B=.24, \beta=.28, t(111)=2.28, p=.025$.

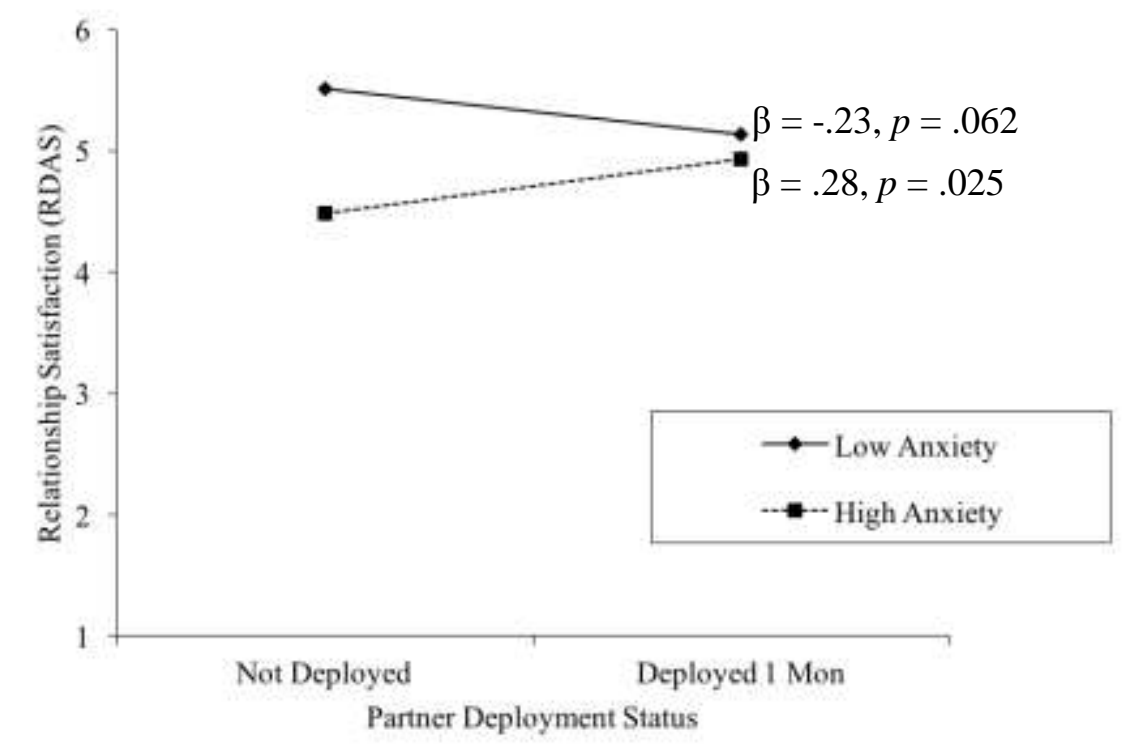

Figure 42. Interaction between attachment anxiety and recent deployment status ( $<1 \mathrm{mon})$ on relationship satisfaction (RDAS).

There were no significant three-way interactions among anxiety, avoidance, and recent deployment status on any of the 19 outcomes.

\section{Attachment Anxiety, Avoidance, and Months Deployed}

Many of the above findings were surprising, specifically that higher levels of attachment anxiety were related to more positive relationship perceptions and indices of well-being. However, individuals higher in attachment anxiety do tend to be more variable in their responses 
to relationship conflicts (Tidwell, Reis, \& Shaver, 1996; Campbell, Simpson, Boldry, \& Kashy, 2005; MacDonald, Wood, and Fabrigar, submitted for publication). In particular, evidence from Phase One, and consistent with findings by Rholes and colleagues (2001), indicates that perceptions of relationship well-being and emotional well-being declines across the separation period for those higher in attachment anxiety. The analyses in this section were intended to test this prediction, that attachment anxiety in particular would interact with the number of months deployed in predicting relationship and intrapersonal outcomes.

Therefore, next I assessed the potential trajectory of the relationships among attachment anxiety, avoidance, and perceptions of one's intrapersonal functioning and relationship perceptions as a function of months deployed. Specifically, I wanted to examine potential linear and curvilinear effects of anxiety and avoidance as a function of months deployed. Recall from earlier that, as outlined in the Canadian Forces Deployment Handbook (Egerton-Graham, 2009), the most challenging stage of the separation is stage 3, occurring during weeks 3-6 of the separation, and consisting of emotional disorganization. Spouse's well-being typically improves when they enter stage 4, recovery and stabilization, which occurs 6-12 weeks into the separation as they have adjusted to their new way of life and distant relationship with their spouse. The next stage after that is the anticipation of homecoming which occurs 1-3 weeks prior to the spouse's arrival home and is fraught with excitement elation and fantasizing.

Although I was interested in examining the potential trajectory of the relationships among anxiety, avoidance, and intrapersonal and interpersonal outcomes as a function of the duration of the deployment, this was challenging with this particular set of data for the following reasons. First, to adequately map the effects of attachment anxiety and avoidance as a function of deployment stages most accurately, I would need to know not only the number of months that 
spouses have been separated from their partners for, but also the projected return date. The return date would allow me to more accurately identify when spouses are likely experiencing the anticipation of homecoming, but unfortunately I didn't have this information. Further, not all deployments are the same in length, so any findings must be interpreted with caution given the variance in deployment length and not being able to adequately infer that the longer the duration, the closer a partner is to returning home.

Nonetheless, assessing the unique and interactive effects of anxiety and avoidance on outcomes as a function of number of months deployed (both linear and curvilinear) gives me an idea about the effects of separation duration on an individual's intrapersonal functioning and relationship perceptions as a function of attachment dimensions. Prior to conducting any analyses, I checked the descriptive properties of the months deployed variable (as I did all variables prior to conducting regressions). This variable was found to be positively skewed (skewness index $=3.57$ ) and with particular descriptive statistics indicating a deviation from normality (e.g., kurtosis index $=12.06$ ), both indicates out of the acceptable ranges proposed by West and colleagues (1995). Therefore, I conducted a normality transformation to the months deployed variable, specifically a Logarithmic ( $\log 10)$ transformation that is appropriate when dealing with a substantial positive skewness. After performing the transformation, this variable had skewness $(=0.40)$ and kurtosis $(=-0.43)$ values that were in an acceptable range, indicating a distribution within normal parameters.

I then conducted multiple linear regressions to assess the unique and interactive effects of (mean centered) attachment anxiety and avoidance, and the number of months deployed (transformed and mean centered) on the same 18 outcomes previously found to be predicted by anxiety in particular. Consistent with previous analyses, relationship length, self-worth, and 
awareness of support programs were included as covariates in these analyses. A simple slopes analyses were used to decompose interactions, examining the effects of deployment months at high (+1 SD) and low (-1 SD) levels of anxiety and avoidance. Similar to previous analyses, I employed an alpha correction, such that the following results were significant if they met the corrected level of significance, $p<.01$. Again, because I was conducting 54 analyses (18 dependent measures and three predictors), .5 findings would be produced by chance with an alpha criterion of .01 . Here, 4 findings were statistically significant.

There was one main effect of months on the desire to eat when stressed $[B=-.52, \beta=-$ $.20, t(186)=-2.48, p=.014]$.

In addition, there was one significant two-way interaction between avoidance and months deployed on stress reported during general military experiences, $B=-.45, \beta=-.23, t(177)=-$ 2.71, $p=.008$ (see Figure 43). At low levels of avoidance, the relationship between months deployed and stress during general military experiences was not significant, $B=.21, \beta=.13$, $t(177)=1.13, p=.262$. However, at high levels of avoidance there was a significant negative relationship between months deployed and stress during general military experiences, $B=-.63, \beta$ $=-.38, t(177)=-2.95, p=.004$.

There was also one significant two-way interaction between attachment anxiety and months deployed on perceived relationship success $[B=-.41, \beta=-.25, t(186)=-3.46, p=.001$; see Figure 44]. At low levels of anxiety, there was a significant positive relationship between months deployed and relationship success, $B=.49, \beta=.25, t(186)=2.72, p=.007$. Further, at high levels of anxiety, there was a significant negative relationship between months deployed and relationship success, $B=-.42, \beta=-.22, t(186)=-2.25, p=.026$. ] 


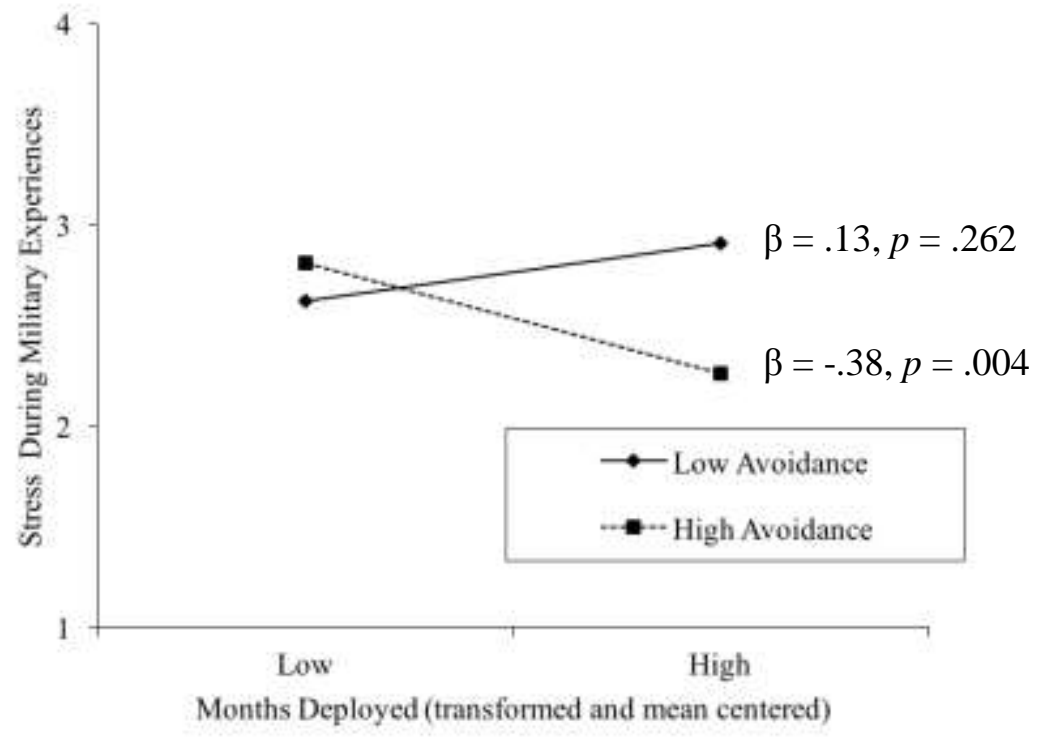

Figure 43. Interaction between attachment avoidance and number of months deployed (transformed and mean centered) on reported stress during general military experiences.

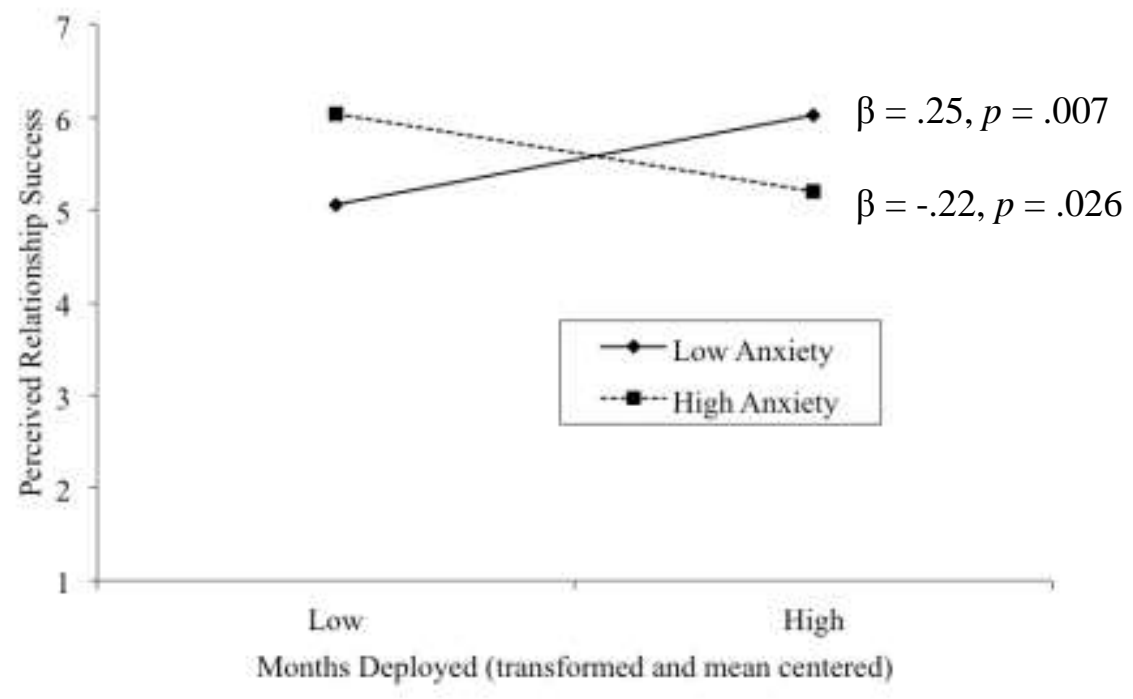

Figure 44. Interaction between attachment anxiety and number of months deployed (transformed and mean centered) on relationship success.

There were two significant higher-order three-way interactions among anxiety, avoidance, and months deployed. Interactions were decomposed using simple slopes analysis 
(Aiken \& West, 1991), examining the effects of months deployed at high (+1 SD) and low (-1 SD) levels of anxiety and avoidance.

First, there was a significant three-way interaction for stress during general military experiences $[B=.28, \beta=.22, t(177)=2.73, p=.007$; see Figure 45$]$. There was only one simple slope that was significant. Specifically, at high levels of avoidance and low levels of anxiety, there was a significant negative relationship between number of months deployed and reported stress during general military experiences, $B=-1.15, \beta=-.69, t(177)=-3.57, p<.001$.

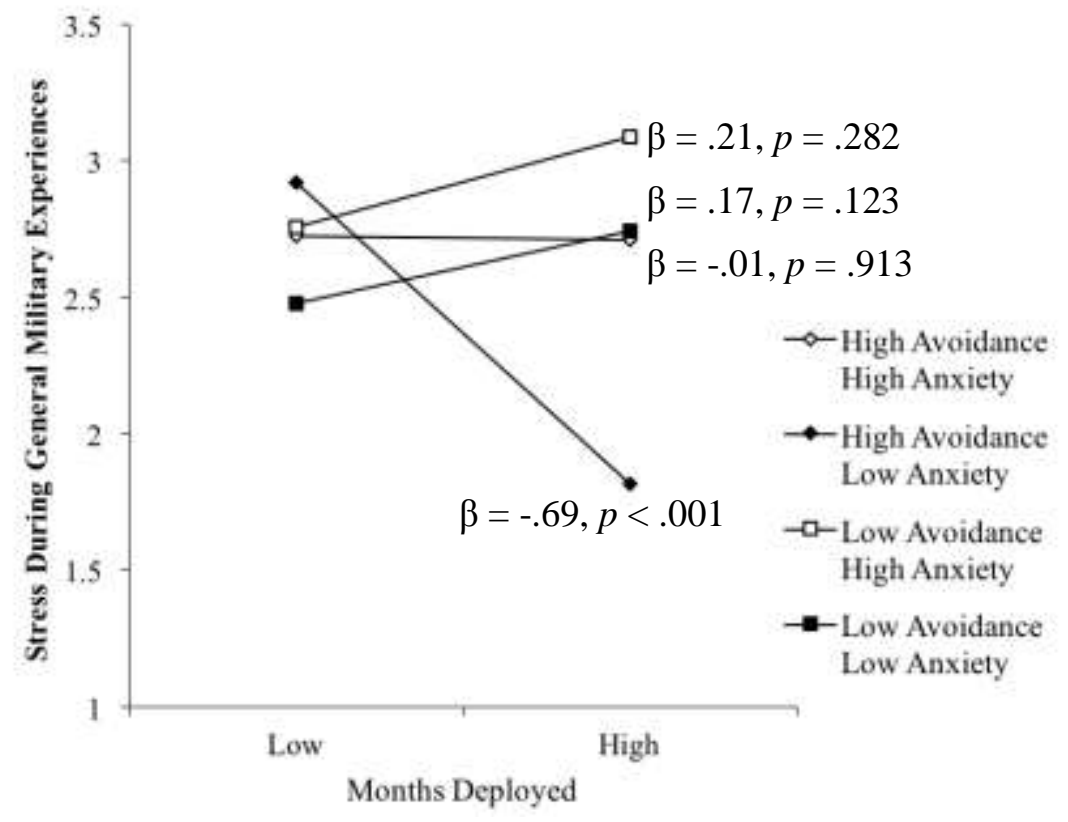

Figure 45. Interaction among attachment anxiety, attachment avoidance, and number of months deployed (transformed and mean centered) on stress during general military experiences.

Second, there was a significant three-way interaction between anxiety, avoidance, and months deployed for coping success $[B=-.36, \beta=-.24, t(185)=-3.29, p=.001$; see Figure 46]. There were two simple slopes that were significant. At high levels of avoidance and low levels of anxiety, there was a significant positive relationship between months deployed and coping success, $B=.97, \beta=.49, t(185)=2.80, p=.006$. Further, at low levels of avoidance and low 
levels of anxiety (the 'secure' group), there was a significant negative relationship between months deployed and coping success, $B=-.47, \beta=-.24, t(185)=-2.46, p=.015$.

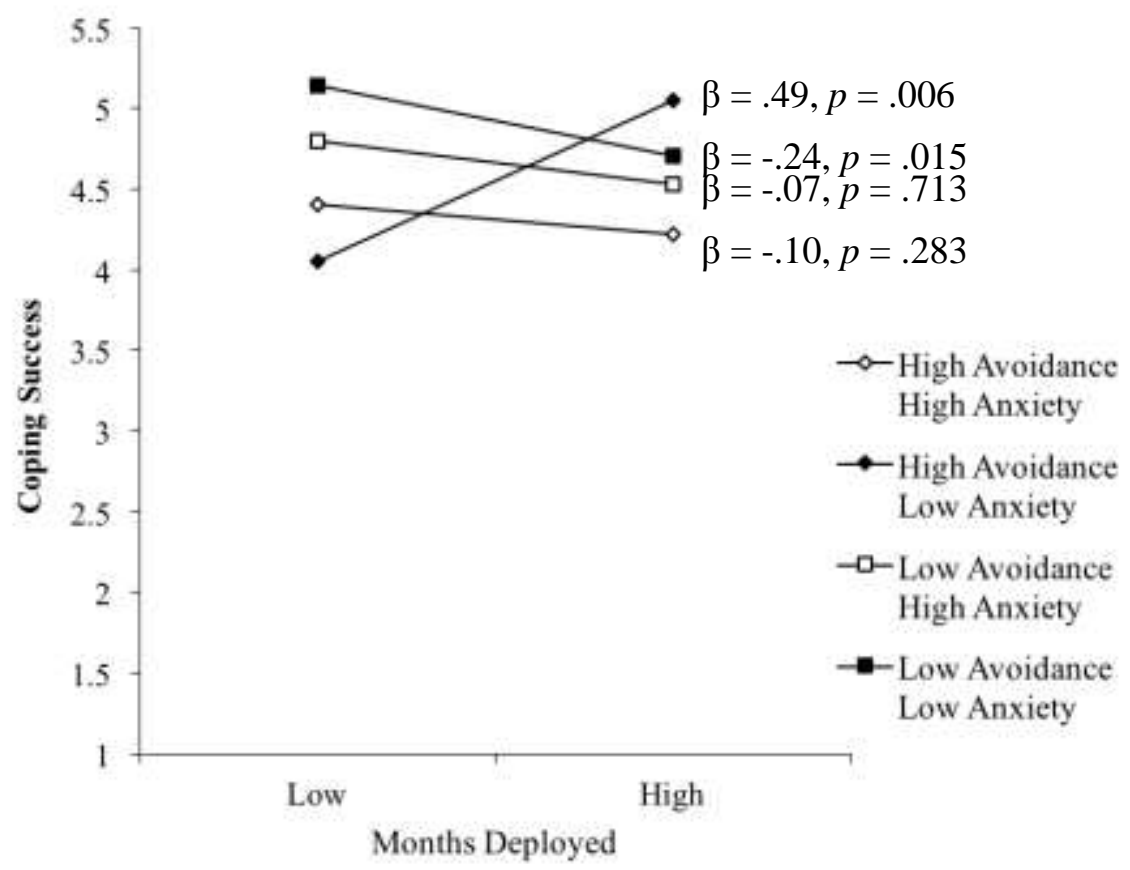

Figure 46. Interaction between attachment anxiety, attachment avoidance, and number of months deployed (transformed and mean centered) on coping success.

\section{Curvilinear Effects of Months Deployed as a Function of Attachment Anxiety and}

\section{Attachment Avoidance}

I was interested in testing whether there were curvilinear effects of months deployed as a function of attachment anxiety and avoidance on the 18 dependent measures. According to the Canadian Forces Deployment Handbook (Egerton-Graham, 2009), the nature of emotional reactivity across the separation is curvilinear. Specifically, spouses progress from more emotional 'reacting' stages (Stages 1-3) to emotional stabilization (Stages 4-5), and then into another reacting stage, Stage 1 of their reunion, which is the anticipation of their partner's homecoming. Therefore, it makes sense to test how the relationships between attachment dimensions and intrapersonal coping and relationship outcomes across the separation might be 
curvilinear in nature. Therefore, I was interested in testing non-linear interactions between anxiety and months deployed, and avoidance and months deployed. To do so, I created the squared function of the transformed and mean-centered months deployed variable as recommended by Aiken and West (1991). Then, I created the months deployed squared by anxiety interaction term, as well as the months deployed squared by avoidance interaction term. I then conducted multiple regressions including the linear and non-linear months deployed main effects, the attachment anxiety and avoidance main effects, the linear and non-linear anxiety and months deployed interaction terms, the linear and non-linear avoidance and months deployed interaction terms, and finally the anxiety and avoidance interaction term. To decompose any significant non-linear interactions, I assessed whether there were curvilinear effects of months deployed at low (-1 SD) and high (+1 SD) levels of anxiety and avoidance. Similar to previous analyses, I employed an alpha correction, such that the following results were significant if they met the corrected level of significance, $p<.01$. Again, because I was conducting 54 analyses (18 dependent measures and three predictors), .5 findings would be produced by chance with an alpha criterion of .01. Here, 2 findings were statistically significant.

There were no curvilinear main effects of months deployed. However, there were significant two-way curvilinear interactions between months deployed squared and attachment anxiety, and months deployed squared and attachment avoidance.

Specifically, there was a significant two-way curvilinear interaction between attachment avoidance and months deployed squared on the use of self-blame as a coping strategy, $B=-.86$, $\beta=-.33 t(182)=-2.77, p=.006$ (see Figure 47). Specifically, at low levels of avoidance the curvilinear relationship between months deployed and use of self-blame was not significant, $B=$ $.47, \beta=.16 t(182)=1.32, p=.189$. However, at high levels of avoidance there was a significant 
curvilinear (inverted $U$ ) relationship between months deployed and use of self-blame as a coping strategy, $B=-1.16, \beta=-.39 t(182)=-3.22, p=.002$.

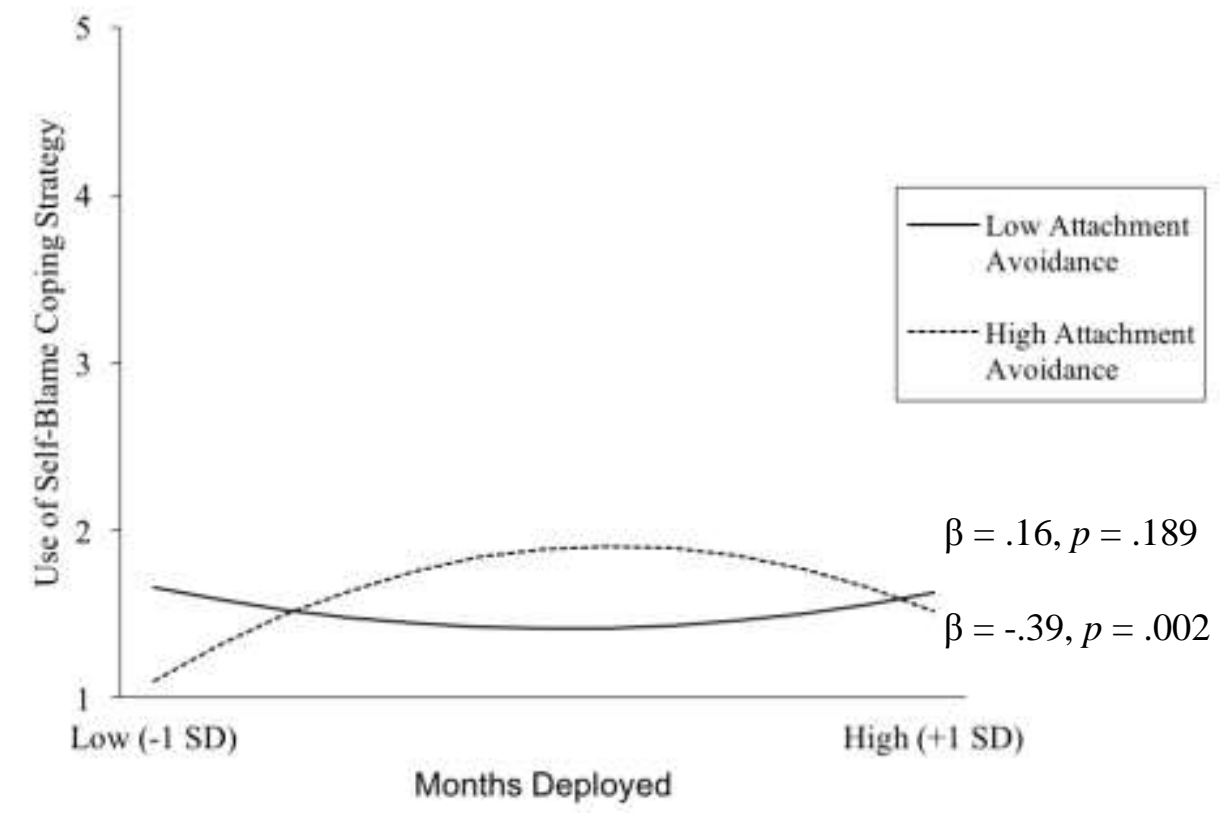

Figure 47. Interaction between attachment avoidance and number of months deployed (transformed and mean-centered) squared on the use of self-blame as a coping strategy.

There was also a significant two-way curvilinear interactions between attachment anxiety and months deployed squared on relationship satisfaction using the QMI measure, $B=-.90, \beta=-$ $.26 t(184)=-2.64, p=.009$. At low levels of anxiety, the curvilinear relationship between months deployed squared and relationship satisfaction was not significant, $B=.88, \beta=.17$ $t(184)=1.80, p=.074$ (see Figure 48). However, at high levels of anxiety there was a significant curvilinear relationship (inverted $\mathrm{U}$ ) between months deployed and relationship satisfaction, $B=$ $-1.10, \beta=-.21 t(184)=-2.32, p=.021$. 


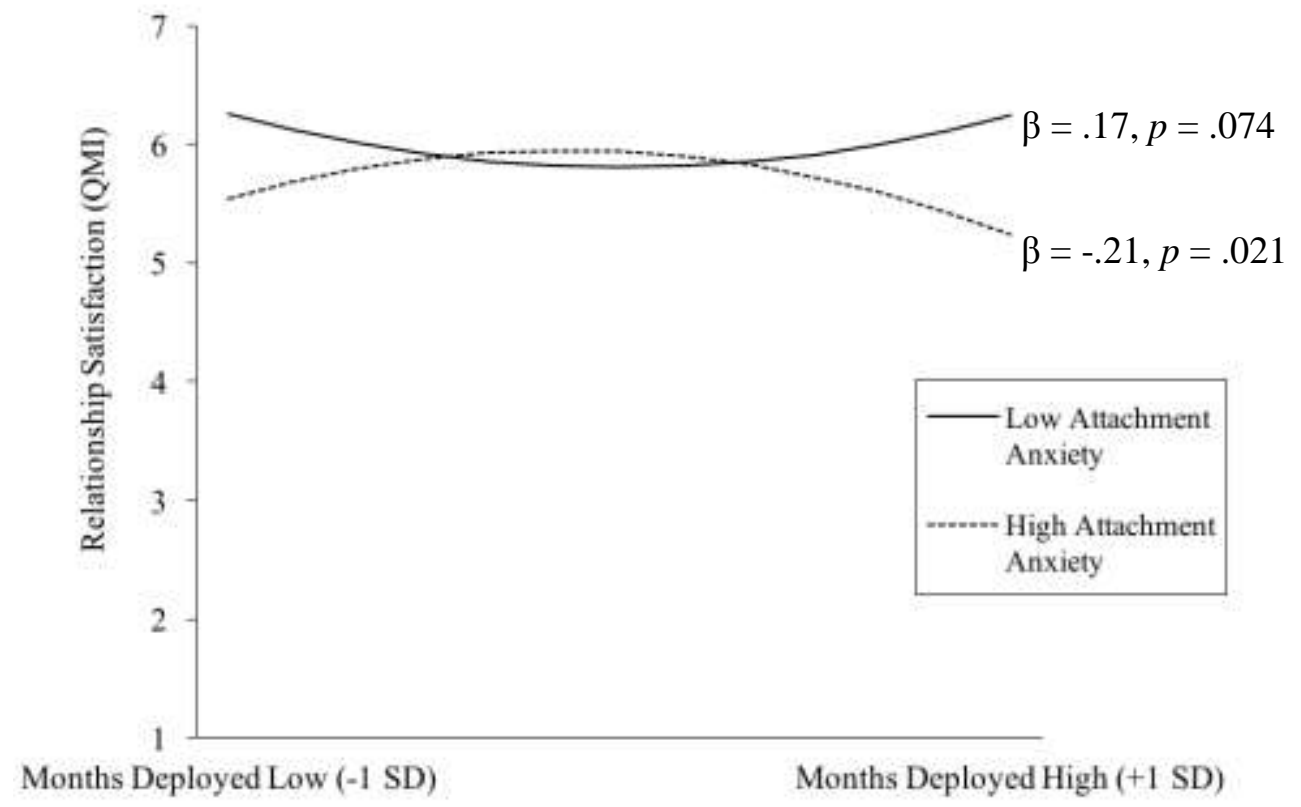

Figure 48. Interaction between attachment anxiety and number of months deployed (transformed and mean-centered) squared on relationship satisfaction (QMI).

\section{Curvilinear Effects of Attachment Anxiety and Attachment Avoidance as a Function of} Months Deployed

It is common in the attachment literature to assume that the effects of attachment dimensions are linear in nature, and, in fact, most researchers are using statistical analyses such as linear regressions that assume these relationships are linear, including here. However, it is important for researchers to start to test these assumptions that we so consistently rely on, so that our theoretical contributions are accurate and informed. Conceptually, it is possible that at some point, being too low in attachment anxiety (no fears of rejection or abandonment), or too low in attachment avoidance (having no discomfort with intimacy and closeness) could be problematic for a relationship. Therefore, as post-hoc analyses, I was interested in testing whether there were curvilinear effects of attachment anxiety and avoidance as a function of months deployed on the 19 dependent measures. Specifically, I was interested in testing non-linear interactions between 
anxiety and months deployed, and avoidance and months deployed. While the bulk of the attachment literature treats the two attachment dimensions as linear, and in fact assumes linear effects of these variables, I wanted to assess the validity of this assumption and test for potential non-linear effects of attachment dimensions in the context of military deployments. To do so, I created the squared function of the mean-centered attachment anxiety and avoidance variables as recommended by Aiken and West (1991). Then, I created the anxiety squared by months deployed interaction term, as well as the avoidance squared by months deployed squared interaction term. I then conducted multiple regressions including the linear and non-linear attachment anxiety and avoidance main effects, the months deployed main effect, the linear and non-linear anxiety and months deployed interaction terms, the linear and non-linear avoidance and months deployed interaction terms, and finally the anxiety and avoidance interaction term. To decompose any significant non-linear interactions, I assessed whether there were curvilinear effects of attachment anxiety and avoidance at low (-1 SD) and high (+1 SD) points of months deployed. Similar to previous analyses, I employed an alpha correction, such that the following results were significant if they met the corrected level of significance, $p<.01$. Again, because I was conducting 54 analyses (18 dependent measures and three predictors), .5 findings would be produced by chance with an alpha criterion of .01 . Here, no findings were statistically significant.

\section{Attachment Anxiety, Avoidance, and Deployment Prep Status}

Logan (1987) argues that one of the most challenging stages is Stage 2 which occurs 1-3 weeks prior to the deployment called detachment and withdrawal and is associated with cognitive, emotional, and relationship difficulties including anxiety, sleep disturbances, regressive behaviours, and ambivalence with displays of intimacy with one's partner. Therefore, 
I predicted that the effects of attachment anxiety on relationship and emotional functioning might be exacerbated for those preparing for a partner's deployment, relative to those not experiencing or preparing for a deployment. Specifically, I examined whether the effects of preparing, versus not preparing, for a deployment moderate the effects of anxiety and avoidance on spousal functioning. To do this, I first collected a different subsample of non-deployed spouses whose partners were not currently preparing for a deployment, and had not returned from a deployment in the past 12 months. Although there were 93 spouses that indicated that their partners were currently preparing for a deployment, 19 were excluded from the subsequent analyses because a) they had also indicated their partners were currently deployed, or b) their partners had returned from a deployment less than 12 months ago. Thus, there were 74 spouses whose partners were currently preparing for a deployment, and were not currently deployed, or had not recently returned from a deployment. For this reason, I collected a sample of 74 nondeployed spouses whose partners had also not returned from a deployment in the past 12 months. This variable was coded such that non-deployed spouses were assigned a value of -1 and spouses preparing for a deployment a value of 1 .

Prior to conducting any analyses, I conducted t-tests (or chi-square tests) first between my non-deployed $(n=74)$ against the larger non-deployed group $(n=1293)$ to ensure that it was a representative sample. Specifically, the groups were compared on the same DVs that were tested between prior groups: being married to same or opposite sex partner, relationship status and duration, gender, age, education, whether the spouse has children, the spouse's employment status, whether the spouse was currently a CAF member, whether they were aware of support programs within their community, their partner's rank and element, and levels of attachment avoidance, or anxiety. The two groups were not statistically different in on any of the DVs. 
Next, I conducted t-tests (or chi-square tests) between the random sample of nondeployed spouses (the 'baseline' group) and the group of spouses currently preparing for a partner's deployment on the same DVs as above. The two groups were not different with respect to being married to the same or opposite sex partner, relationship status, gender, education, whether they have children, whether the spouse is current a serving CAF member, awareness of support programs, or levels of attachment avoidance or anxiety. There were, however, some differences between these two groups. Specifically, the group preparing for a deployment was younger $(M=36.12, S D=7.63)$ than the baseline group $[M=38.76, S D=8.24 ; t(171)=2.63, p$ $=.033]$, had been with their partners for less time $(M=10.57, S D=6.95)$ than the baseline group $[M=14.77, S D=8.41 ; t(166)=4.20, p<.001)$, and had deployed more times in the past five years $(M=1.33, S D=1.48)$ relative to the baseline group $(M=.89, S D=.96 ; t(109)=-$ $2.18, p=.032$ ). Given the above, age, relationship length, number of times deployed in the past 5 years were included as covariates in subsequent analyses.

I then conducted multiple linear regressions to examine the unique and interactive effects of attachment anxiety, avoidance, and deployment prep status on the $18 \mathrm{DV}$ s that were significant for the main effects of anxiety, consistent with the analyses for deployment status, recent deployment status, and months deployed. The following were included as covariates given the above findings: age, relationship length, number of times deployed in the past five years, along with self-worth (which correlated with anxiety), relationship length, and awareness of support programs (which both correlated with avoidance) again consistent with previous analyses. Simple slopes analyses were used to decompose interactions, examining the effects of deployment prep status at high (+1 SD) and low (-1 SD) levels of anxiety and avoidance. Similar to previous analyses, I employed an alpha correction, such that the following results 
were significant if they met the corrected level of significance, $p<.01$. Again, because I was conducting 54 analyses (18 dependent measures and three predictors), .5 findings would be produced by chance with an alpha criterion of .01. Here, no findings were statistically significant.

\section{Attachment Anxiety, Avoidance, and Recent Reunion Status}

According to the Canadian Forces Deployment Handbook (Egerton-Graham, 2009), couples are in 'reacting' stages 2-6 weeks ('reintegration') and 6-12 weeks ('renegotiation') post-reunion. I wanted to assess whether attachment anxiety and avoidance moderated the effects of post-reunion experiences relative to a 'baseline' or couples not experiencing a reunion or separation. With the Phase One reunion group, I found that the effects of attachment anxiety on some relationship outcomes were moderated by time reunited. For example, for highly anxious individuals their perceptions of their interactions with their partners declined as a function of time reunited. However, there was no relationship between quality of interactions and time reunited for those lower in attachment anxiety. This is consistent with the findings of Rholes and colleagues (2001) who found that for anxious spouses, their perceptions of their partner's support declined across the transition into parenthood. This is also consistent with my findings in this study reported earlier, that individuals higher in attachment anxiety, while initially report higher adjustment and well-being at the first stage of the separation relative to a baseline group, these individuals showed declines in relationship perceptions and indices of well-being as the separation progressed. Therefore, I predicted that, in line with the previous findings from this Phase, attachment anxiety would be associated with increased perceptions of relationship functioning and intrapersonal well-being for those recently reunited with their partners, relative to those not experiencing a reunion. Theoretically this makes sense, that if 
individuals high in attachment anxiety are dependent on their partners for emotional comfort, reassurance, and validation, experience increased distress in response to partner separations, and desire greater levels of closeness in their relationships, it could be argued that those high in attachment anxiety should respond more positively to this honeymoon period. Such individuals might report feeling more emotionally connected to their partners, more satisfied in their relationships, and experience improved emotional well-being relative to those lower in attachment anxiety.

On the other hand, highly avoidant individuals may be less satisfied with their partners' behaviour during the Elation period as they learn to cope with their partners being physically closer. Having one's partner home would naturally increase levels of intimacy in the relationship making avoidant individuals uncomfortable and might potentially lead to conflict and more unpleasant partner interactions. This would be consistent with work by Tidwell, Reis, and Shaver (1996) that found that avoidant individuals reported less intimacy, enjoyment and positive emotions with their partners, and that avoidant individuals would structure social activities in ways that minimize intimacy and closeness.

To test these predictions, I compared the group of spouses who had been reunited with their partner from a military deployment for $1-2$ months $(n=76)$ and were not preparing for another deployment, to a group of spouses who were not currently experiencing a reunion or separation with their partner, were not preparing for a deployment and had not deployed in the past 12 months $(\mathrm{n}=1293)$. Given the differences in sample sizes, and consistent with previous analyses, I randomly selected a subsample of 76 individuals from this latter group to use for comparison against the reunion group. 
I then conducted t-tests (and chi-square where appropriate) to determine whether the random sample of non-reunion spouses were representative of the larger non-reunion group on key demographic and predictor variables. Results from these analyses showed that these two groups were statistically different on only one out of the 14 variables. Specifically, the random non-reunion sample was more likely to have children $(M=1.00, S D=.18)$ relative to the recent reunion group $(M=.97, S D=.00), t(1236)=-6.34, p<.001$. However, the two groups were not statistically different on the following variables: whether the spouses were married to a same or opposite sex partner, relationship status, relationship length, gender, age, education, number of partner deployments in the past five years, partner's military element, partner's rank, whether the spouse is currently a CAF member, awareness of support programs, or levels of avoidance or anxiety. Therefore, this sample was fairly representative of the larger non-reunion group.

I also conducted the same t-tests (and chi-square tests) to compare scores on the same variables above between the baseline and reunion groups to rule out potential confounding variables. The two groups were statistically different on two variables. First, the recently reunited group had experienced more deployments in the past five years $(M=3.59, S D=3.60)$ relative to the non-reunited group $[M=0.75, S D=.90 ; t(83)=-6.63, p<.001)$. Second, the proportion of spouses who were currently CAF members (regular or reserve force) or civilians was not consistent across the two groups, $\chi^{2}(2, N=149)=8.84, p=.012$. Specifically, the recently reunited group had fewer at-home spouses who were regular force members and more that were civilian than expected, and the non-reunited group has more spouses who were regular force members and fewer spouses who were not military members than expected. For the above reasons, number of deployments in the past five years and whether the spouses were CAF members were included as covariates in the following analyses along with self-worth, 
relationship length, and awareness of support programs consistent with prior analyses. Simple slopes analyses (Aiken \& West, 1991) were used to decompose any interactions, assessing the effect of recent reunion status at high (+1 SD) and low (-1 SD) levels of attachment anxiety and avoidance. Similar to previous analyses, I used the corrected level of significance of $p<.01$. Again, because I was conducting 54 analyses (18 dependent measures and three predictors), .5 findings would be produced by chance with an alpha criterion of .01. Here, 2 findings were statistically significant.

There was a main effect of recent reunion status on self-distraction as a coping strategy such that those who were recently reunited with their partners engaged in greater self-distraction than those not recently reunited, $B=.27, \beta=.33, t(99)=2.58, p=.011$. There was also a main effect for life readiness, such that those who were recently reunited with their partners reported more life readiness than those not recently reunited, $B=.26, \beta=.32, t(99)=2.69, p=.008$.

Next, there was a significant two-way interaction between anxiety and recent reunion status on perceived relationship success, $B=.41, \beta=.46, t(99)=3.29, p=.001$ (see Figure 49). At low levels of anxiety, there was a significant negative relationship between reunion status and perceived relationship success such that those who were recently reunited reported less relationship success than those not recently reunited, $B=-.39, \beta=-.40, t(99)=-2.14, p=.035$. However, at high levels of anxiety there was a significant positive relationship between recent reunion status and perceived relationship success such that spouses recently reunited with their partners reported higher levels of relationship success relative to those not recently reunited, $B=$ $.52, \beta=.54, t(99)=3.29, p=.001$. 


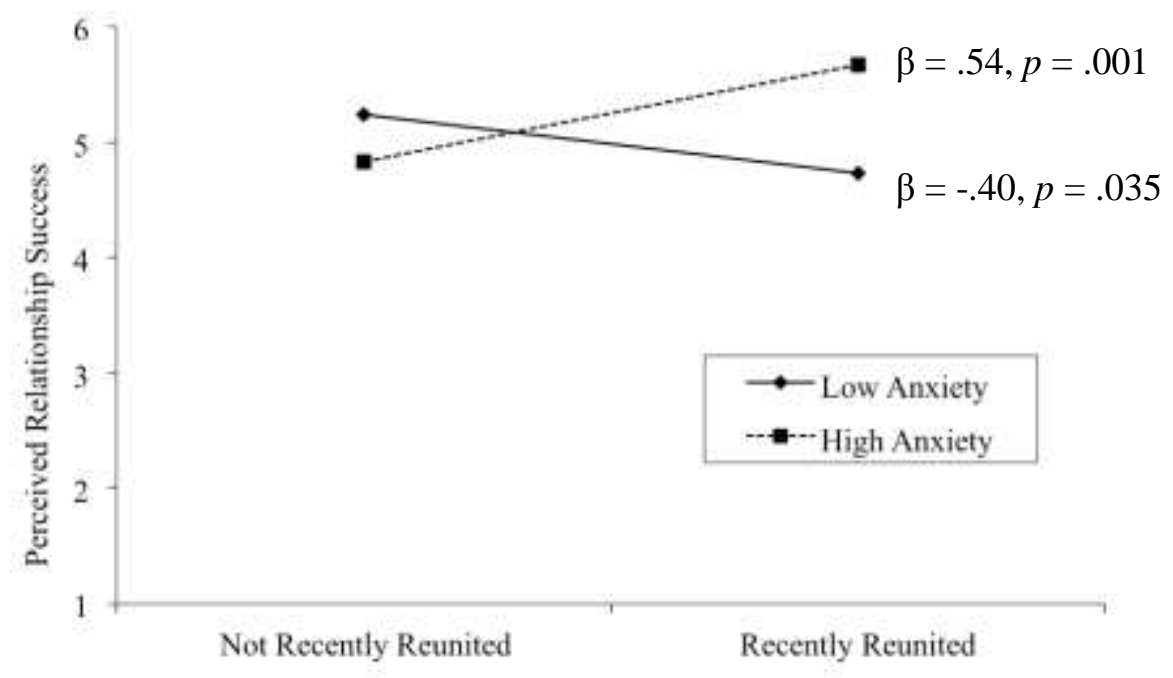

Figure 49. Interaction between attachment anxiety and recent reunion status on perceived relationship success.

There was also a significant two-way interaction for relationship consensus, $B=.24, \beta=$ $.38, t(100)=2.73, p=.007$ (see Figure 50). Again, at low levels of anxiety the relationship between recent reunion status and relationship consensus was not significant, $B=-.23, \beta=-.35$, $t(100)=-1.86, p=.066$. However, at high levels of anxiety there was a significant positive relationship between recent reunion status and relationship consensus such that spouses recently reunited with their partners reported higher levels of relationship consensus relative to those not recently reunited, $B=.30, \beta=.44, t(100)=2.57, p=.012$. 


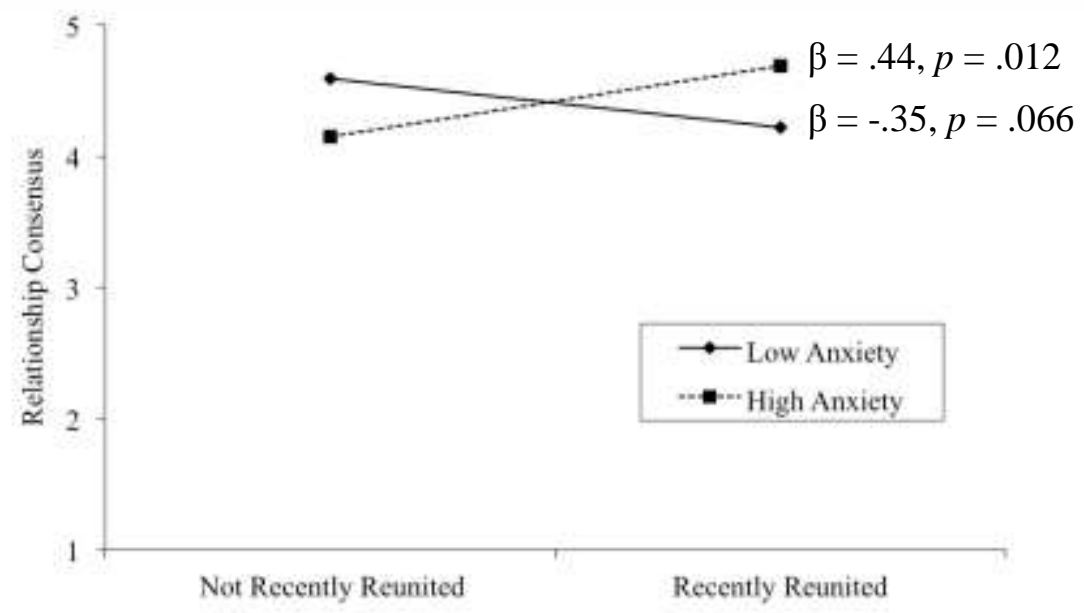

Figure 50. Interaction between attachment anxiety and recent reunion status on relationship consensus.

\section{Attachment Anxiety, Avoidance, and Months Reunited}

Next, I was interested in assessing the potential trajectory of the relationships among attachment anxiety, avoidance, and perceptions of one's intrapersonal functioning and relationship perceptions as a function of months reunited. Specifically, I wanted to examine potential linear and curvilinear effects of anxiety and avoidance as a function of months reunited. According to the Canadian Forces Deployment Handbook (Egerton-Graham, 2009), couples typically move through the following stages during a partner's deployment reunion: elation (0-2 weeks post-reunion), reintegration (2-6 weeks post-reunion), renegotiation (6-12 weeks post-reunion), integration and acceptance (12-16 weeks post-reunion), and stabilization (16 weeks post-reunion and onwards). As previously mentioned, the reintegration and renegotiation stages are typically reported to be the most problematic with spouses reporting the following emotional and behavioural responses more than other stages: uncertainty, withdrawal, anxiety, fatigue, frustration, avoidance, resentment, denial, reluctance, and other physical symptoms. Therefore, the typical response pattern seems to be curvilinear, with couples 
reporting more adaptive emotional and behavioural responses during earlier $(<2$ weeks postreunion), and later (> 12 weeks), stages relative to the middle stages (2-12 weeks post-reunion). However, some couples tend to 'roller coaster' back and forth through particular stages. It is possible that attachment anxiety and avoidance can help to explain potential patterns (curvilinear or linear) in outcomes as a function of months reunited. Theoretically, attachment anxiety should predict a decline in relationship satisfaction and emotional well-being across the reunion period potentially as they start to feel as though their partners' behaviours are not living up to what they had expected (Rholes et al., 2001; Simpson \& Rholes, 1998). This would be in line with work by Cafferty and colleagues (1994) that found that, on average four months after a deployment reunion, spouses who were insecurely attached to their partners reported more conflict and less relationship satisfaction relative to secure spouses.

I first assessed potential linear relationships among attachment anxiety, avoidance, and months reunited on the 18 outcomes previously found to be predicted by attachment anxiety. Given that stabilization post-reunion typically occurs 16 weeks post-reunion and onward, I used the 6 month ( 24 week) mark as the 'stabilization' or final reunion stage end point, rather than the 4 month (16 week) end point, to ensure that I captured enough variability for the stabilization stage. According to the Canadian Forces Deployment Handbook (Egerton-Graham, 2009), couples might progress through some stages slower than others, making some stages longer in duration, which is dependent on the success of one's coping.

I then conducted multiple linear regressions to assess the unique and interactive effects of (mean centered) attachment anxiety and avoidance, and the number of months deployed (also mean centered) on the same 18 outcomes previously found to be predicted by anxiety. Consistent with previous analyses, relationship length, self-worth, and awareness of support 
programs were included as covariates in these analyses. These analyses would determine potential linear effects of attachment dimensions as a function of months reunited. I decomposed interactions using simple slopes analyses (Aiken \& West, 1991), examining the effects of months reunited at low (-1 SD) and high (+1 SD) levels of attachment avoidance and anxiety. Consistent with previous analyses, I employed an alpha correction, such that the following results were significant if they met the corrected level of significance, $p<.01$. Again, because I was conducting 54 analyses (18 dependent measures and three predictors), .5 findings would be produced by chance with an alpha criterion of .01 . Here, 2 findings were statistically significant.

There were no significant main effects of months reunited on any of the 19 outcomes. However, there a significant two-way interaction between avoidance and months reunited on life mastery, $B=-.12, \beta=-.46, t(131)=-3.60, p<.001$ (see Figure 51). At low levels of avoidance, there was a significant positive relationship between months reunited and feeling a sense of mastery over one's life, $B=.10, \beta=.34, t(131)=2.49, p=.014$. However, at high levels of avoidance there was a significant negative relationship between months reunited and mastery, $B=-.12, \beta=-.43, t(131)=-2.91, p=.004$.

In addition, there was a significant two-way interaction between attachment anxiety and months reunited on life mastery, $B=.08, \beta=.31, t(131)=2.65, p=.009$ (see Figure 52). At low levels of anxiety, there was a significant negative relationship between months reunited and mastery, $B=-.10, \beta=-.35, t(131)=-2.51, p=.013$. However, at high levels of anxiety the relationship between months reunited and mastery was not significant, $B=.08, \beta=.27, t(131)=$ $1.68, p=.094$ 


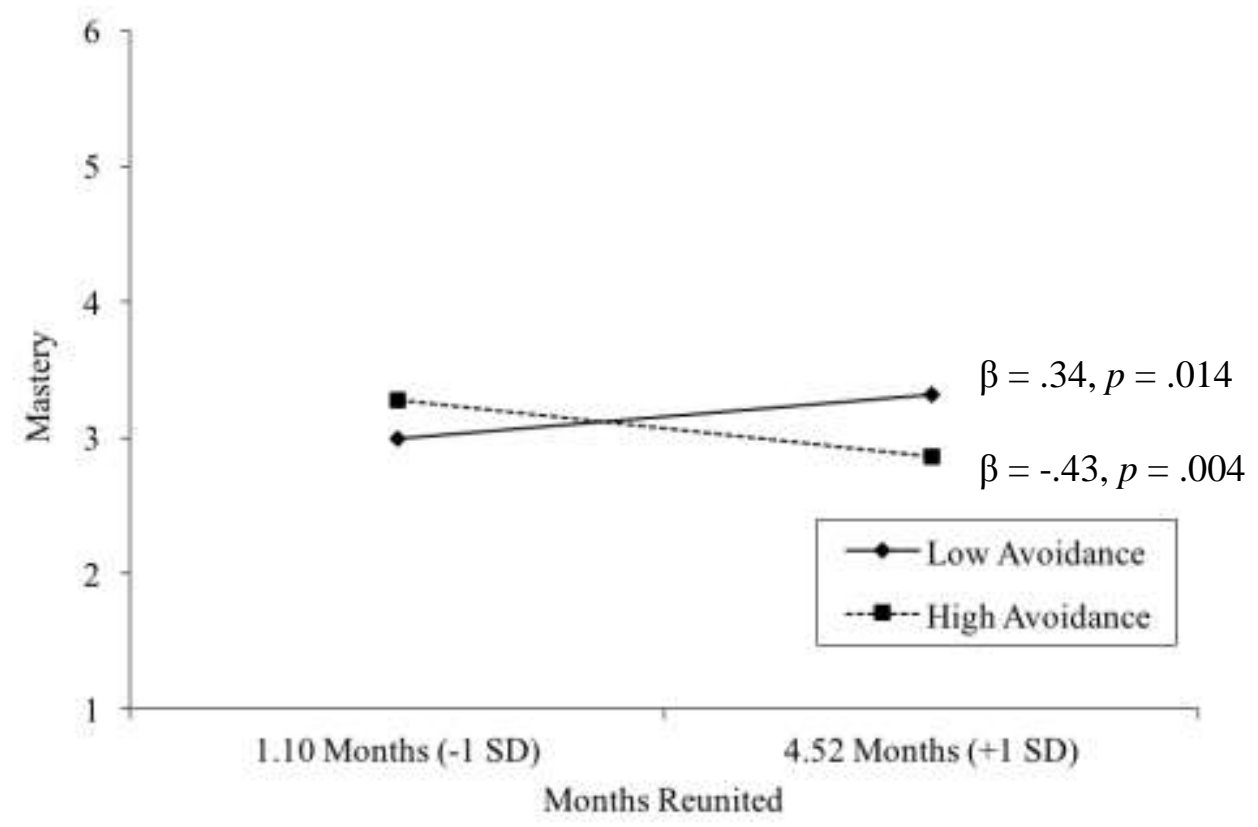

Figure 51. Interaction between attachment avoidance and months reunited on life mastery.

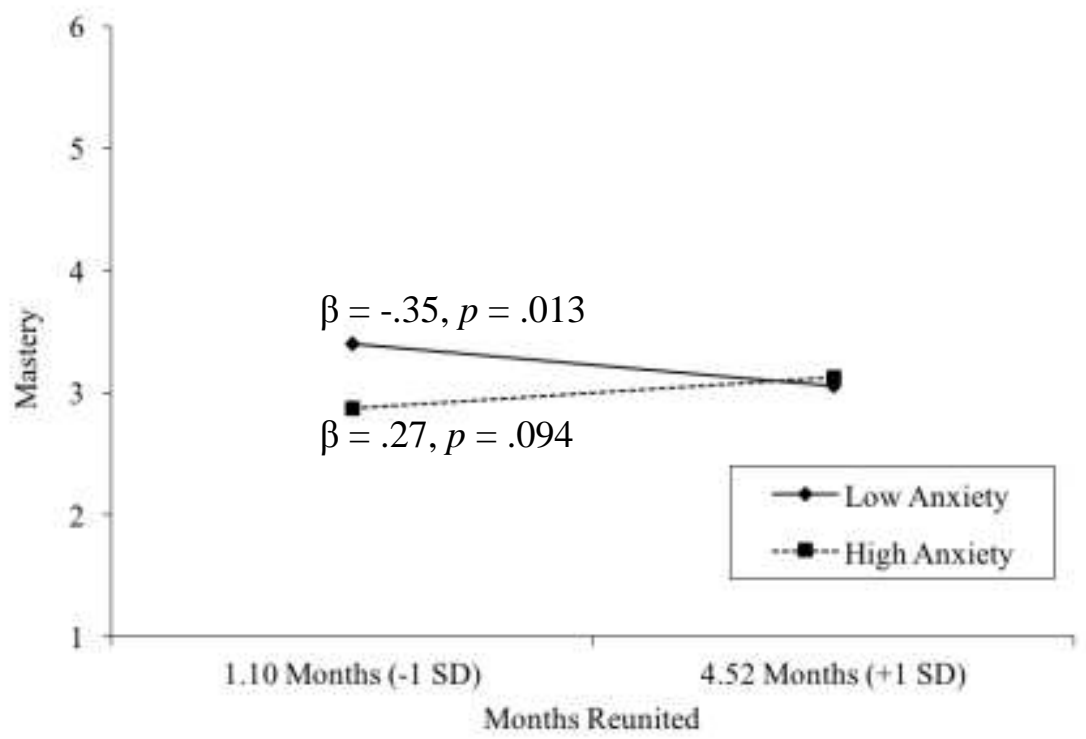

Figure 52. Interaction between attachment anxiety and months reunited on life mastery. 


\section{Curvilinear Effects of Months Reunited as a Function of Attachment Anxiety and}

\section{Attachment Avoidance}

As previously reported in this Phase, I found evidence to suggest that the effects of attachment dimensions on relationship and intrapersonal adjustment to deployment experiences might not always follow a linear trajectory across the deployment. Therefore, I had reason to believe that the same patterns might be true for the reunion period, and next tested whether there were curvilinear effects of months reunited as a function of attachment anxiety and avoidance on the 18 dependent measures. Specifically, I was interested in testing non-linear interactions between anxiety and months reunited, and avoidance and months reunited. To do so, I created the squared function of the mean-centered months reunited variable as recommended by Aiken and West (1991). Then, I created the months reunited squared by anxiety interaction term, as well as the months reunited squared by avoidance interaction term. I then conducted multiple regressions including the linear and non-linear months reunited main effects, the attachment anxiety and avoidance main effects, the linear and non-linear anxiety and months reunited interaction terms, the linear and non-linear avoidance and months reunited interaction terms, and finally the anxiety and avoidance interaction term. To decompose any significant non-linear interactions, I assessed whether there were curvilinear effects of months reunited at low (-1 SD) and high (+1 SD) levels of anxiety and avoidance. I employed an alpha correction, such that the following results were significant if they met the corrected level of significance, $p<.01$.

Because I was conducting 54 analyses (18 dependent measures and three predictors), .5 findings would be produced by chance with an alpha criterion of .01. Here, 4 findings were statistically significant. 
While there were no curvilinear main effects of months reunited, there was a significant two-way interaction between months reunited squared and attachment avoidance on relationship success, $B=-.10, \beta=-.52, t(129)=-2.95, p=.004$ (see Figure 53). Specifically, at low levels of avoidance the curvilinear relationship was not significant between months reunited and relationship success, $B=.08, \beta=.26, t(129)=1.94, p=.055$. However, at high levels of avoidance there was a significant curvilinear relationship (inverted $U$ ) between months reunited and relationship success, $B=-.12, \beta=-.39, t(129)=-2.90, p=.004$.

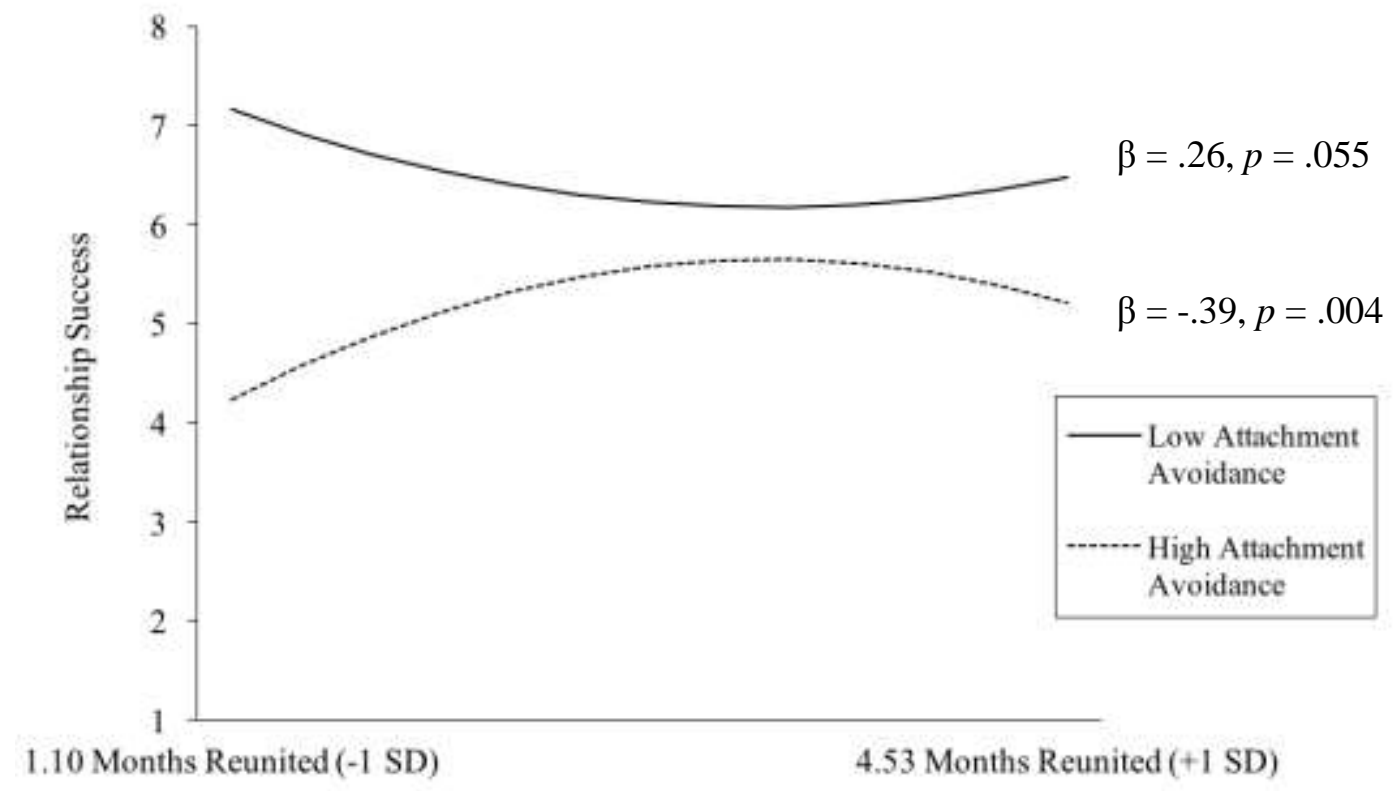

Figure 53. Interaction between attachment avoidance and months reunited squared on relationship success.

Next, there was a significant two-way interaction between attachment avoidance and months reunited squared on relationship satisfaction using the QMI measure, $B=-.13, \beta=-.49$, $t(129)=-2.79, p=.006$ (see Figure 54). Similar to the results for relationship success, at low levels of avoidance the curvilinear relationship was not significant between months reunited and relationship success, $B=.10, \beta=.25, t(129)=1.89, p=.061$. However, at high levels of 
avoidance there was a significant curvilinear relationship (inverted $\mathrm{U}$ ) between months reunited and relationship success, $B=-.15, \beta=-.36, t(129)=-2.69, p=.008$.

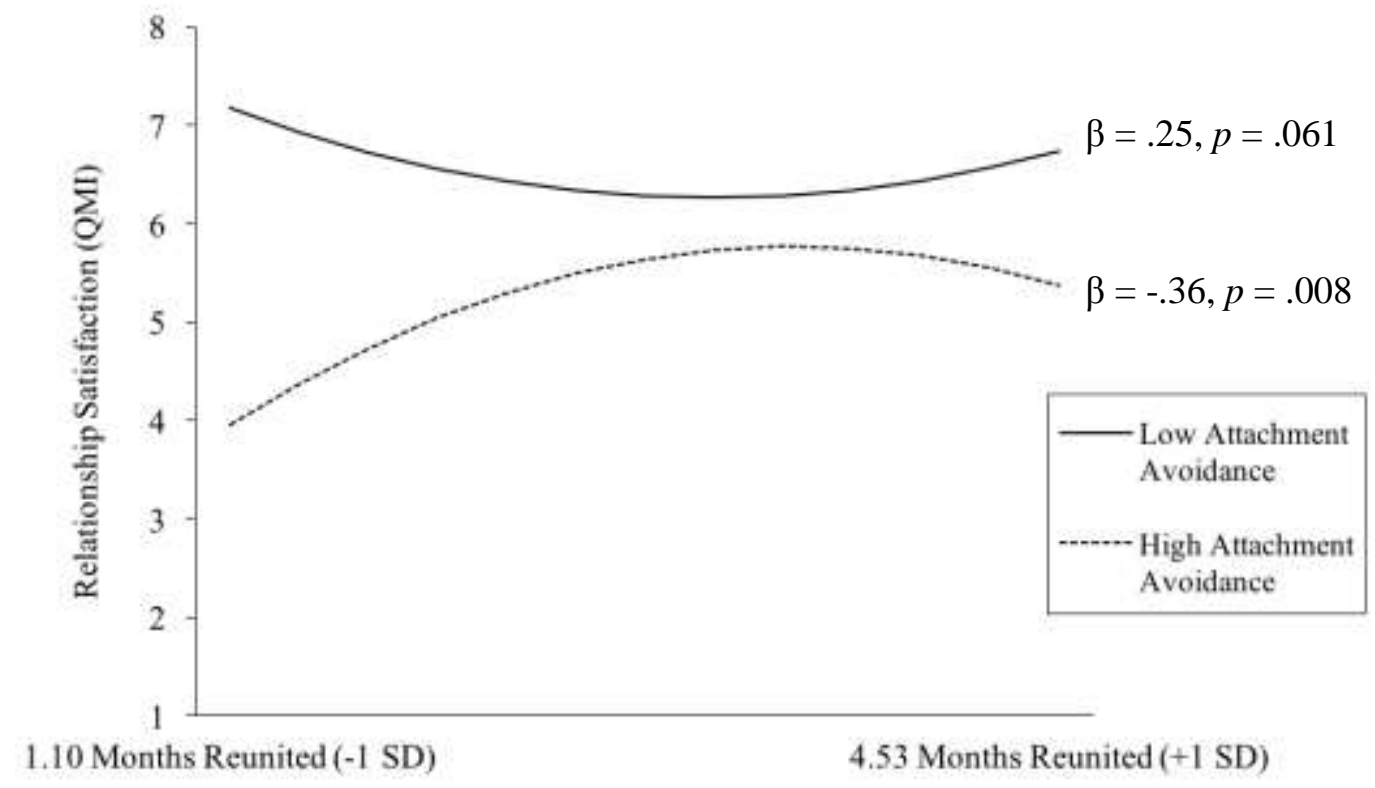

Figure 54. Interaction between attachment avoidance and months reunited squared on relationship satisfaction using the QMI measure.

Next, there were significant two-way interactions between attachment anxiety and months reunited squared. There was a significant two-way interaction for life readiness, $B=$ $.11, \beta=-.57, t(129)=-2.68, p=.008$ (see Figure 55). Specifically, at low levels of anxiety the curvilinear relationship was not significant between months reunited and life readiness, $B=.08$, $\beta=.29, t(129)=1.46, p=.147$. However, at high levels of anxiety there was a significant curvilinear (inverted $\mathrm{U}$ ) relationship between months reunited and life readiness, $B=-.16, \beta=$ $.58, t(129)=-3.11, p=.002$. 


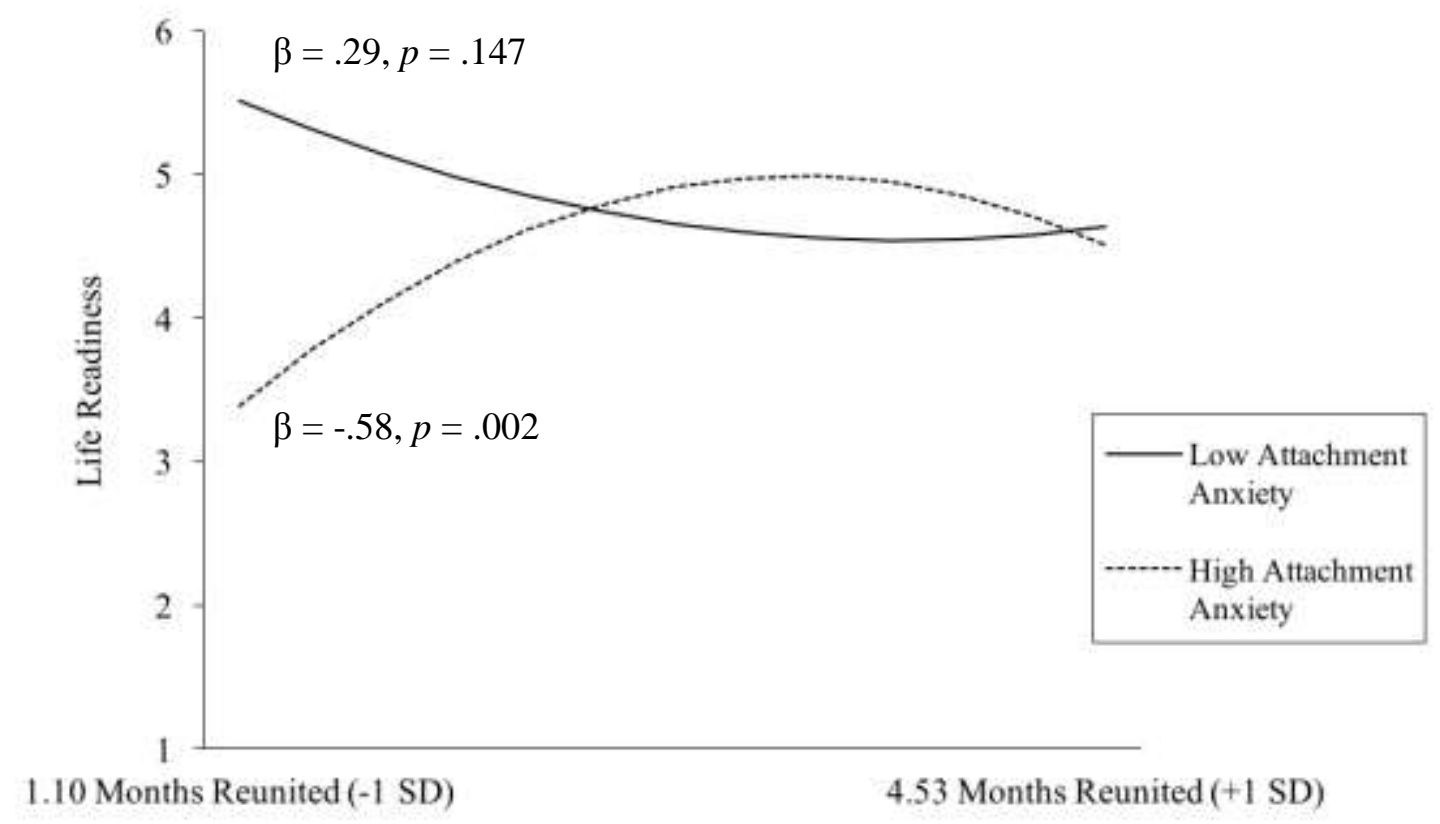

Figure 55. Interaction between attachment anxiety and months reunited squared on life readiness.

There was also a significant two-way interaction for relationship success, $B=.09, \beta=$ $.46, t(129)=2.94, p=.004$ (see Figure 56). This time, at low levels of anxiety there was a significant curvilinear relationship (inverted $\mathrm{U}$ ) between months reunited and relationship success, $B=-.12, \beta=-.42, t(129)=-2.87, p=.005$. In addition, at high levels of anxiety there was a significant curvilinear relationship (U shaped) between months reunited and relationship success, $B=.08, \beta=.28, t(129)=2.06, p=.042$. 


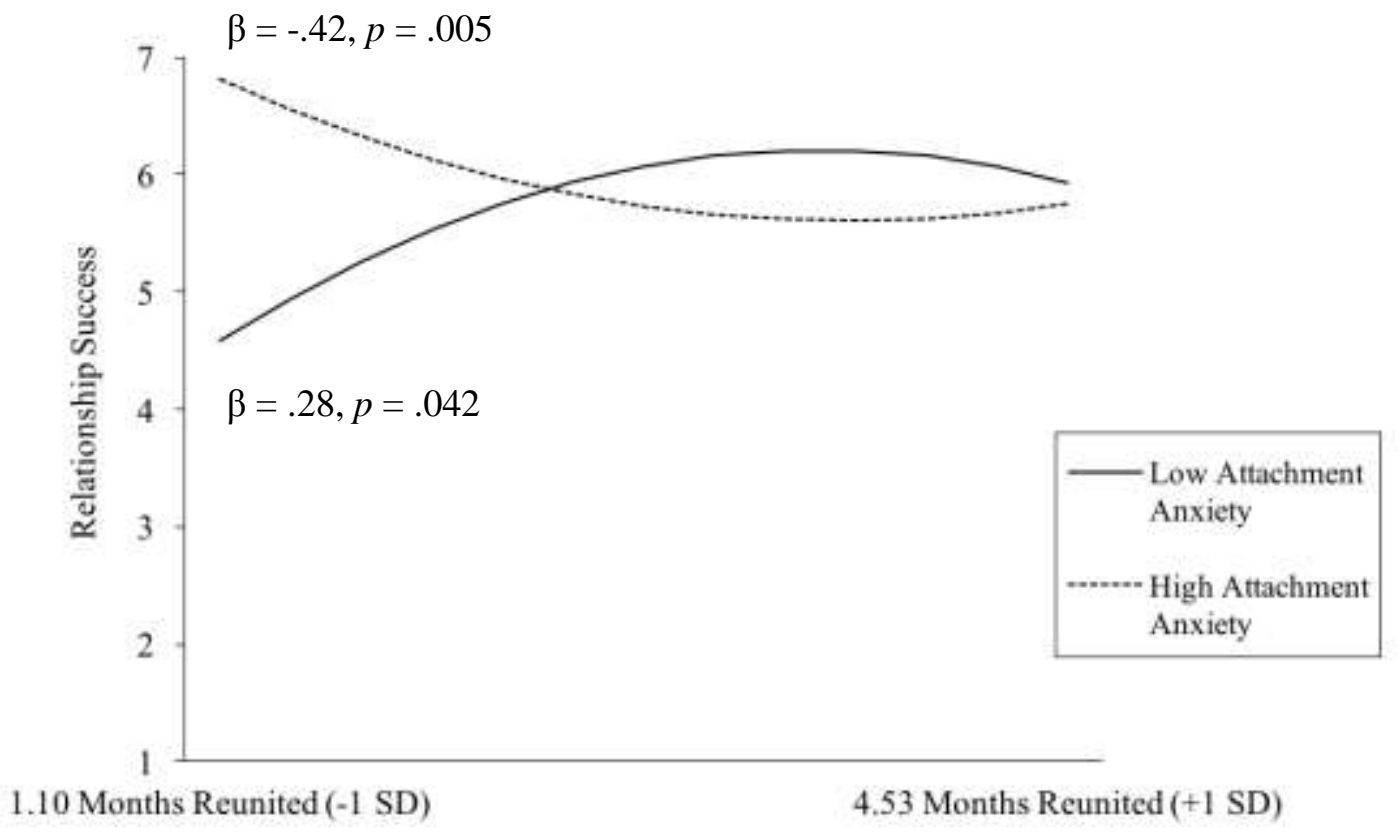

Figure 56. Interaction between attachment anxiety and months reunited squared on relationship success.

\section{Mediation Models for Attachment Anxiety and Related Outcomes}

Finally, I was interested in identifying potential cognitive mechanisms that might explain the relationships between attachment anxiety and intrapersonal outcomes and relationship perceptions post-hoc. Specifically, I was interested in more closely examining the relationships between attachment anxiety and coping and relationship measures that were significantly related to anxiety in previous models. The nine coping outcomes that were significantly related to anxiety were amount psychological distress, subjective well-being, the ability to handle daily hassles, life mastery, coping success, cognitive fitness, emotional fitness, and the use of selfblame and self-distraction coping strategies. In addition, the six relationship outcomes that were significantly related to anxiety were perceived relationship support, perceived relationship success, relationship satisfaction (QMI and RDAS), relationship consensus, and relationship cohesion. 
For the following mediation analyses, I used Preacher and Hayes' (2008) bootstrapping method (here using 1000 samples) which produces an empirically derived CI of the indirect effect estimate. If zero is not included in the CI, one can conclude that the indirect effect is significant. I performed these analyses using an SPSS multiple mediator macro (available from afhayes.com) that allows for the inclusion of control variables into the tests of indirect effects.

The two measures that best capture one's ability to manage stressors (coping capacity), rather than coping outcomes themselves, are emotional fitness (having an optimistic outlook) and cognitive fitness (use of planning and critical thinking when facing adversity). Further, the specific coping strategies that were related to anxiety were the use of self-blame and selfdistraction. I was interested in identifying which of these four variables best acted as a mediator between attachment anxiety and coping outcomes and relationship perceptions. When each of these four variables was entered into the mediation model, the other three variables were treated as dependent measures. Therefore, the predictor in all models was attachment anxiety, one of the four fitness/coping strategies as the mediator, and with 14 dependent measures.

\section{Self-Blame Coping Strategy as a Mediator}

When the use of self-blame as a coping strategy was treated as the mediator in the relationship between attachment anxiety on the 14 dependent measures, there was partial mediation for nine of the 14 dependent measures, full mediation for the use of self-distraction as a coping strategy, but no evidence of mediation for four variables (cognitive fitness, relationship support, consensus, and cohesion). Therefore, for self-blame there was evidence of mediation for six out of eight coping outcomes, and three out of six relationship outcomes. Self-Distraction Coping Strategy as a Mediator 
When the use of self-distraction as a coping strategy was treated as the mediator in the relationship between attachment anxiety and the 14 dependent measures, there was partial mediation for seven variables, but no evidence of mediation for seven variables. Specifically, there was partial mediation for six out of eight coping measures and one out of six relationship measures.

\section{Cognitive Fitness as a Mediator}

Next, when cognitive fitness was treated as the mediator in the relationship between attachment anxiety and the 14 dependent measures, there was partial mediation for 11 out of 14 variables, but no evidence of mediation for two variables (self-blame coping strategy, relationship support, relationship satisfaction using the RDAS measure). Specifically, there was partial mediation for seven out of eight coping measures, and three out of six relationship variables.

\section{Emotional Fitness as a Mediator}

When emotional fitness was treated as the mediator in the relationship between attachment anxiety and the 14 dependent measures, there was partial mediation for 13 out of 14 measures, and with no mediation for relationship satisfaction (RDAS). Therefore, emotional fitness partially mediated eight out of eight coping outcomes, and partially mediated five out of six relationship outcomes.

In summary, emotional fitness was the best mediator in the relationships between attachment anxiety and coping outcomes, specifically psychological distress, subjective wellbeing, the ability to handle daily hassles, life mastery, coping success, cognitive fitness, and the use of self-blame and self-distraction coping strategies (see Table 8). However, none of the coping strategies or coping fitness indices completely mediated the relationships between 
attachment anxiety and relationship outcomes. While emotional fitness acted as a mediator for four out of five of the relationship outcomes, I wanted to investigate whether an index of relationship perceptions better mediated the relationships between attachment anxiety and relationship outcomes. Rholes and colleagues (2001) found that the perceptions of partner support declined across the transition into parenthood for highly anxious spouses. Therefore, in line with these findings, I was interested in assessing whether perceptions of partner support (here called relationship support) mediated the relationships between attachment anxiety and relationship satisfaction (measured using RDAS and QMI), perceived relationship cohesion, perceived relationship consensus, and relationship success. As predicted, relationship support partially mediated the relationships between attachment anxiety and relationship satisfaction (QMI and RDAS), relationship consensus, and relationship success, and fully mediated the relationship between attachment anxiety and relationship cohesion (see Table 9).

Table 8

Mediation Models for Relationships Between Attachment Anxiety and Coping Outcomes as Mediated by Emotional Fitness

\begin{tabular}{lccccc}
\hline Dependent Measure & $\begin{array}{c}a \\
\mathrm{X} \rightarrow \mathrm{Y})\end{array}$ & $\begin{array}{c}b \\
(\mathrm{M} \rightarrow \mathrm{Y})\end{array}$ & $\begin{array}{c}c \\
\text { (indirect) }\end{array}$ & $\begin{array}{c}c^{\prime} \\
\text { (direct) }\end{array}$ & $\begin{array}{c}\text { 95\% BC-CI of } \\
\text { indirect effect }\end{array}$ \\
\hline Psychological distress & - & $-3.83^{*}$ & $2.33^{*}$ & $1.40^{*}$ & $.71-1.17$ \\
Subjective well-being & - & $.67^{*}$ & $-.26^{*}$ & $-.10^{*}$ & $-.21-(-.13)$ \\
Ability to handle hassles & - & $.37^{*}$ & $-21^{*}$ & $-.12^{*}$ & $-.11-(-.07)$ \\
Life mastery & - & $.25^{*}$ & $-.17^{*}$ & $-.11^{*}$ & $-.08-(-.05)$ \\
Coping success & - & $.55^{*}$ & $-.26^{*}$ & $-.12^{*}$ & $-.17-(-.11)$ \\
Cognitive fitness & - & $.38^{*}$ & $-.13^{*}$ & $-.04^{*}$ & $-.11-(-.07)$ \\
Self-blame coping strategy & - & $-.23^{*}$ & $.30^{*}$ & $.24^{*}$ & $.04-.07$ \\
Self-distraction coping strategy & - & $-.06^{*}$ & $.12^{*}$ & $.11^{*}$ & $.01-.03$ \\
\hline Note. X here is attachment anxiety, and M is emotional fitness. BC-CI refers to the bias- \\
corrected confidence interval of the indirect effect estimate. \\
$*$ indicates $p<.05$.
\end{tabular}


Table 9

Mediation Models for Relationships Between Attachment Anxiety and Relationship Perceptions as Mediated by Perceived Relationship Support

\begin{tabular}{lccccc}
\hline Dependent Measure & $\begin{array}{c}a \\
\mathrm{X} \rightarrow \mathrm{Y})\end{array}$ & $\begin{array}{c}b \\
(\mathrm{M} \rightarrow \mathrm{Y})\end{array}$ & $\begin{array}{c}c \\
\text { (indirect) }\end{array}$ & $\begin{array}{c}c^{\prime} \\
\text { (direct) }\end{array}$ & $\begin{array}{c}95 \% \text { BC-CI of } \\
\text { indirect effect }\end{array}$ \\
\hline Relationship satisfaction (QMI) & $-.21^{*}$ & $.81^{*}$ & $-.29^{*}$ & $-.12^{*}$ & $-.23-(-.13)$ \\
Relationship satisfaction (RDAS) & - & $.34^{*}$ & $-.17^{*}$ & $-.10^{*}$ & $-.10-(-.05)$ \\
Relationship consensus & - & $.24^{*}$ & $-.14^{*}$ & $-.09^{*}$ & $-.07-(-.03)$ \\
Relationship cohesion & - & $.28^{*}$ & $-.07^{*}$ & -.01 & $-.08-(-.04)$ \\
Relationship success & - & $.59^{*}$ & $-.34^{*}$ & $-.08^{*}$ & $-.30-(-.22)$ \\
\hline
\end{tabular}

\section{Discussion}

Attachment dimensions seem to be important predictors of a broad range of intrapersonal and relationship outcomes. Theoretically, individuals higher in attachment anxiety perceive emotions as being congruent with attachment goals and therefore tend to exaggerate them, as well as exaggerate the presence and seriousness of threats both related to, and unrelated to, attachment needs. (Feeney, 1998). In line with this reasoning, I found that attachment anxiety was negatively associated with a number of indices capturing emotional well-being including decreased ability to handle daily demands, decreased subjective well-being, less mastery over one's life, and greater use of more maladaptive and emotion-focused coping strategies such as self-blame and self-distraction from the stressor. Attachment anxiety was also associated with greater reported stress during general military experiences, indicating that highly anxious individuals perceive military experiences to be more stressful than less anxious individuals. Attachment anxiety also predicted less resiliency in the face of adversity including less of an ability to think critically in the face of a stressor (cognitive fitness), and less of an ability to enjoy life and remain optimistic about one's situation (emotional fitness). Further, some of these negative effects of attachment anxiety on intrapersonal outcomes were exacerbated for those also 
low in avoidance, particularly for the use of emotion-focused coping strategies and social support, but also life readiness. In other words, the individuals who seem to struggle the greatest with coping with adversities and managing emotions are those low in avoidance but higher in attachment anxiety, relative to those low in avoidance and low in anxiety. These findings extend the current literature on the relationship between attachment anxiety and coping strategies and subjective well-being which have not previously reported such interactions (Birnbaum, Orr, Mikulincer, \& Florian, 1997; Meyer, Olivier, and Roth, 2005). Finally, attachment anxiety was negatively associated with a number of relationship perceptions such as perceiving less support from one's partner, being less satisfied in their relationships (on a few measures), and disagreeing more with one's spouse on various issues. However, most of these effects were exacerbated for those high in attachment avoidance. Indeed, for those low in attachment avoidance, higher levels of anxiety were associated with more negative relationship perceptions. This is consistent with work by Collins and Feeney (2004) who found that fearfully attached individuals (high in attachment anxiety and avoidance) perceived their partners' personal messages of encouragement to be less supportive, controlling for the independent ratings of their partners' support in the messages. Further, these findings generally support a number of studies showing that attachment security is associated with greater relationship satisfaction and communication styles (e.g., Rholes et al., 2001; Simpson, 1990; Kobak \& Hazen, 1991).

On the other hand, individuals with higher levels of attachment avoidance should view negative emotions and opportunities to express vulnerability as being incompatible with their need for self-reliance (Feeney, 1998). Specifically, highly avoidant individuals are known to effectively mask verbal and nonverbal emotional expressions, to suppress emotion-related action tendencies, or beliefs that one can manage emotions effectively on their own (e.g., Kobak, Cole, 
Forenz-Gillies, Fleming, \& Gamble, 1993; Mikulincer \& Shaver, 2003). Further, attachment avoidance is typically associated with distancing coping, however some studies have also shown emotion-focused strategies used by highly avoidant individuals when managing conflicts with their romantic partners (e.g., Lussier, Sabourin, \& Turgeon, 1997). Interestingly, I found that attachment avoidance was indeed related to a type of distancing coping, but also with a number of indices of intrapersonal well-being and coping capacity. Specifically, attachment avoidance was associated with decreased subjective well-being, the extent to which they can manage stressors, having a more negative life outlook, and decreased ability to think critically in the face of a stressor. Consistent with their working models of distrust, and consistent with work by Collins and Read (1990), attachment avoidance was also associated with negative relationship perceptions including perceiving less support from one's partner, being less satisfied (on a few measures), disagreeing more with one's spouse on various issues, and feeling as though one engages in fewer activities with one's partner.

Mikulincer, Gillath, and Shaver (2002) demonstrated that the adult attachment system becomes activated under conditions of attachment-related threat (e.g., potential or experienced separation from an attachment figure) as well as general threat (e.g., stress or physical danger). Such conditions automatically and implicitly activate thoughts of attachment figures that can motivate an individual to increase (or in the case of avoidance, decrease) physical or psychological proximity to them. In other words, working models of attachment should regulate attachment behaviour (e.g., proximity seeking strategies, distress-signalling, or distancing). Indeed, individual differences in attachment behaviour should be most pronounced under stressful conditions in the social or physical environment (Simpson \& Rholes, 1994). Specifically, under threatening conditions (characterized by increased stress or emotional 
reactivity), attachment anxiety should be associated with hyperactivating strategies such as increased distress, emotion-focused coping strategies, and perhaps even more negative relationship perceptions (Birnbaum et al., 1997; Fraley \& Shaver, 1998; Mayseless et al., 1996; Meyer et al., 2005). However, attachment avoidance should be associated with deactivating strategies aimed at promoting interpersonal distance when the attachment system is activated such as potential reports of increased well-being (or a lack of decreased well-being), use of distancing coping strategies, and more negative relationship perceptions (Fraley \& Shaver, 1997; Fraley \& Shaver, 1998; Mayseless et al., 1996; Simpson et al., 1992). There is the possibility, however, that breakdowns in emotional well-being and coping capacity will be observed with increases in attachment avoidance, if environmental conditions are characterized by high enough levels of stress such that deactivating strategies are less effective (Mikulincer \& Florian, 1998). Therefore, I next assessed the extent to which attachment anxiety and avoidance interacted with a number of deployment-related moderating variables that should correspond to variations in levels of experienced threat.

\section{Deployment Separation Findings}

First, I focused on deployment-related separations. I first reasoned that the effects of attachment dimensions on intrapersonal coping and relationship outcomes might be moderated by deployment status, or whether the spouses were currently separated from their partners by a military deployment. However, there were no main effects of deployment status on any coping indices. Therefore, overall whether or not one's spouse is deployed isn't a great predictor of overall well-being. Accordingly, I did not find that deployment status moderated any effects of attachment anxiety. However, deployment status did moderate some effects of attachment avoidance. Specifically, low levels of avoidance were associated with greater use of self- 
distraction as a coping strategy and the ability to think critically in the face of a stressor for those whose spouses were deployed, relative to those whose spouses were not deployed. This is some support for the notion that increased levels of avoidance are associated with some breakdowns in emotional well-being and coping capacity, particularly for those also high in anxiety.

As outlined in the Canadian Forces Deployment Handbook (Egerton-Graham, 2009), the most challenging stage of the separation is stage 3, occurring during weeks 3-6 of the separation, and consisting of emotional disorganization. Using this logic, I compared responses of spouses who had most recently ( $\leq 1$ month) separated from their partners, and therefore most likely experiencing emotional disorganization, relative to an equal number of spouses who were not currently separated from their partners. There was more support for this variable as a threatrelated moderator as spouses whose partners were recently deployed reported a decreased ability to handle daily hassles relative to those not separated from their partners. Interestingly, I found that at lower levels of avoidance, those who were recently separated from their partners reported greater perceived relationship support, relationship success, relationship satisfaction, and feeling as though one agrees with one's partner on various issues, and an increased ability to think critically in the face of a stressor, relative to those whose partners were not deployed. However, at higher levels of avoidance, those who were recently separated from their partners reported a decreased ability to think critically in the face of stressors, decreased perceived relationship support, relationship satisfaction, and feeling more as though one does not agree with one's partner on a number of issues, relative to those not currently separated. In other words, low levels of avoidance are associated with greater emotional and relationship functioning, and higher levels of avoidance are associated with more relationship difficulties, during a high-stress period typically associated with emotional difficulties. 
The nature of the relationships among attachment anxiety and intrapersonal and relationship outcomes also differed depending on whether one was recently separated from one's partner or not. Specifically, lower levels of attachment anxiety were associated with greater psychological distress, a decreased ability to think critically when facing stressors, and decreased perceived relationship success for those recently separated relative to those not separated. However, higher levels of anxiety were associated with more positive coping and relationship perceptions for those recently separated relative to those not separated such as a greater ability to think critically when faced with a stressor, greater perceived relationship success, and relationship satisfaction. In other words, those lower in anxiety are struggling more with intrapersonal coping and relationship functioning during the emotional disorganization period relative to a baseline, whereas those with higher levels of anxiety seem to be experiencing better coping and relationship outcomes during this period.

In general, these findings suggest that those struggling the greatest with the high-stress Stage 3 (initial 3-6 weeks after separation) relative to a baseline state (partner not deployed) are those with higher levels of attachment avoidance or greater discomfort with emotional closeness, and those with lower levels of attachment anxiety, or less fears of rejection and abandonment. Further, those adjusting better during this high-stress period are those with higher levels of attachment anxiety, or greater fears of rejection and abandonment, and those with low levels of attachment avoidance, or more comfort with emotional intimacy. Attachment anxiety is associated with needs for reassurance and validation, which corresponds with a desire for emotional dependency. Therefore, it is possible that those with greater needs for connection and reassurance in this initial period are faring better because they might be making more efforts to connect with their partners, and establish intimacy, or are engaging in more proximity-seeking 
and intimacy-building activities with their partners. This might be reflected in increased attempts to contact one's partner, more intimate conversations involving support seeking and provision, and successfully gaining that reassurance they so desperately desire. It is also possible that their partners are more accepting of the spouse's 'neediness' during this stage, relative to those at home together, and so highly anxious, or less avoidant, individuals are receiving the attention they desire normally but perhaps do not receive to the same extent. However, those with greater levels of discomfort with emotional intimacy and dependency, or less fears of rejection and abandonment are less motivated to connect with their partners, and maintain intimacy, from afar. In other words, tendencies to engage in de-activating strategies to reduce psychological dependency on one's partner, or less of a concern about rejection and abandonment, might lead these individuals to not reach out to one's partner as often or as intimately, to not provide nor seek as much emotional support from one's partner, and maintain more of a 'out of sight out of mind' mentality.

Admittedly, some of these findings were surprising, specifically that higher levels of attachment anxiety were related to more positive relationship perceptions and indices of wellbeing. Although, individuals higher in attachment anxiety do tend to be more variable in their responses to relationship conflicts (Campbell, Simpson, Boldry, \& Kashy, 2005; MacDonald, Wood, \& Fabrigar, submitted for publication; Simpson, \& Kashy, 2005; Tidwell, Reis, \& Shaver, 1996). In particular, evidence from Phase One, and findings by Rholes and colleagues (2001), indicates that perceptions of relationship well-being and emotional well-being declines across the separation period for those higher in attachment anxiety. The analyses in this section were intended to test this prediction, that attachment anxiety in particular would interact with the number of months deployed in predicting relationship and intrapersonal outcomes. I tested both 
linear and curvilinear functions of time as a moderator of attachment avoidance, anxiety, and their interaction.

There were not many effects of attachment avoidance or attachment anxiety as a linear function of time deployed on outcomes, but one finding consistent with those from Phase One, was that low levels of anxiety were associated with increases in perceived relationship success over time, whereas higher levels of anxiety were associated with decreases in perceived relationship success across the separation. Finally, there were some areas where attachment anxiety and avoidance interacted as a function of time. Specifically, for those with lower levels of attachment anxiety and avoidance, perceptions of the extent to which one is coping successfully, decreased across the separation. Also, for those with higher levels of avoidance and lower levels of anxiety, perceptions of stress experienced during general military experiences became more positive over time. Finally, for those with higher levels of avoidance but lower levels of anxiety, coping success improved over the course of the separation. Given that these findings were not consistent across more measures of coping outcomes, and the same slope was not significant across measures, it is premature to speculate about the importance of these interactions to predict relationship and intrapersonal functioning. However, they do illustrate that interactions between these constructs are important to assess and account for, so that accurate interpretations of results, and accurate theoretical contributions, can be made.

According to the Canadian Forces Deployment Handbook (Egerton-Graham, 2009), the nature of emotional reactivity across the separation is curvilinear. Specifically, spouses progress from more emotional 'reacting' stages (Stages 1-3) to emotional stabilization (Stages 4-5), and then into another reacting stage, Stage 1 of their reunion, which is the anticipation of their partner's homecoming. Therefore, it makes sense to test how the relationships between 
attachment dimensions and intrapersonal coping and relationship outcomes across the separation might be curvilinear in nature. I found only weak evidence for curvilinear effects of attachment anxiety and avoidance across the separation (2 significant effects out of 54). Specifically, at high levels of attachment avoidance the use of self-blame during the separation assumed an inverted U shape (starting low, increasing over time, and ending low near the end of the separation). This suggests that those high in avoidance tended to use self-blame (a typically attachment anxietyrelated coping strategy) in the middle of a separation period, but less so during those transition periods. In contrast, higher levels of attachment anxiety were only predictive of curvilinear patterns across time for relationship satisfaction. Specifically, for those with higher levels of attachment anxiety, perceived relationship satisfaction assumed an inverted-U shape. In other words, for those with higher levels of anxiety, relationship satisfaction was less positive near the beginning and end of the separation surveys, but more positive near the middle of the separation. This might be perceived as being inconsistent with the finding that for those higher in attachment anxiety, relationship quality is higher for those recently separated relative to those not recently separated. However, it is possible that relationship quality indices continue to improve after the 1 month mark, but then at some point decline, and then begin to improve again closer to the homecoming. Again, because there was only one curvilinear finding for attachment anxiety on relationship quality as a function of time, it really is too premature to speculate about its significance.

It is common in the attachment literature to assume that the effects of attachment dimensions are linear in nature, and, in fact, most researchers are using statistical analyses such as linear regressions that assume these relationships are linear, including here. However, it is important for researchers to start to test these assumptions that we so consistently rely on, so that 
our theoretical contributions are accurate and informed. Conceptually, it is possible that at some point, being too low in attachment anxiety (no fears of rejection or abandonment), or too low in attachment avoidance (having no discomfort with intimacy and closeness) could be problematic for a relationship. Therefore, I wanted to test for the potential non-linear effects of attachment dimensions in the context of military deployments. While none of the interactions met the corrected level of statistical significance $(<.01)$, there were some interactions that were statistically significant at the .05 level (not reported), demonstrating that it may be possible for some effects of attachment dimensions to be curvilinear in nature, and that future researchers should continue to test curvilinear effects, and the validity of the assumption of linearity of attachment dimensions.

Logan (1987) argued that one of the most challenging stages is Stage 2 which occurs 1-3 weeks prior to the deployment called detachment and withdrawal and is associated with cognitive, emotional, and relationship difficulties including anxiety, sleep disturbances, regressive behaviours, and ambivalence with displays of intimacy with one's partner. Therefore, I predicted that the effects of attachment anxiety on relationship and emotional functioning might be exacerbated for those preparing for a partner's deployment, relative to those not experiencing a deployment, given that highly anxious individuals should by nature be more fearful of an experience of abandonment relative to less anxious individuals. While I found some support that this period is challenging for spouses, with deployment preparation status associated with decreased subjective well-being, there were no other main effects of deployment preparation status on other coping indices. Further, I found no support for the notion that attachment dimensions (or their interaction) moderate spousal functioning during this period. 


\section{Deployment Reunion Findings}

Next, I focused on deployment-related reunion experiences. According to the Canadian Forces Deployment Handbook (Egerton-Graham, 2009), couples are in 'reacting' stages 2-6 weeks ('reintegration') and 6-12 weeks ('renegotiation') post-reunion. I wanted to assess whether attachment anxiety and avoidance moderated the effects of post-reunion experiences in a group of spouses who were likely in the middle of these stages (1-2 months reunited) relative to a 'baseline' or couples not experiencing a reunion or separation. I did not find much support for the notion that recent reunion status moderates relationships among attachment dimensions and spousal functioning. Regarding the findings that were significant, and in line with my predictions and the findings from Phase One, those with higher levels of attachment anxiety reported higher levels of relationship success, and the extent to which one agrees with their partner on various issues, for those recently reunited relative to those not recently reunited. This is consistent with findings from Phase one that attachment anxiety was associated with increased perceptions of relationship functioning and intrapersonal well-being for those recently reunited with their partners, relative to those not experiencing a reunion. Theoretically this makes sense, that if individuals high in attachment anxiety are dependent on their partners for emotional comfort, reassurance, and validation, experience increased distress in response to partner separations, and desire greater levels of closeness in their relationships, those high in attachment anxiety should respond more positively to this honeymoon period. Such individuals might report feeling more emotionally connected to their partners, more satisfied in their relationships, and experience improved emotional well-being relative to those lower in attachment anxiety.

Next, I was interested in assessing the potential trajectory of the relationships among attachment anxiety, avoidance, and perceptions of one's intrapersonal functioning and 
relationship perceptions as a function of months reunited (both linear and curvilinear trajectories). While I did not find much support for the notion that attachment dimensions interact with months deployed in a linear fashion, I did find more support for curvilinear effects across time according to levels of attachment avoidance and anxiety. Specifically, for those with higher levels of attachment avoidance, perceptions of relationship success and relationship satisfaction were lower near the beginning of the reunion, increased over time, and then decreased after the 4.5-month mark (inverted-U shape). These findings are in line with my predictions that highly avoidant individuals might have difficulty in reconnecting with one's partner emotionally, and that having one's partner home would naturally increase levels of intimacy in the relationship making avoidant individuals uncomfortable and might potentially lead to conflict and more unpleasant partner interactions (Tidwell et al., 1996). However, these findings indicate that some degree of adjustment eventually takes place for highly avoidant individuals such that over time their relationship perceptions improve.

Regarding attachment anxiety, for those with lower levels of anxiety, perceptions of life mastery decreased across the reunion. The simple slopes for higher levels of attachment anxiety did not reach statistical significance. There were also curvilinear effects of attachment anxiety on intrapersonal and relationship indices across time. For example, at higher levels of attachment anxiety, perceptions of life readiness were lower near the beginning of the reunion period, improved over time, but then declined again near the 4.5-month mark (inverted-U shape). In contrast, perceptions of relationship success were U-shaped in nature. At higher levels of attachment anxiety, perceptions of relationship success were initially higher, decreased over time, and then started to increase again near the 4.5 -month mark. These findings are again consistent with those from Phase One, where attachment anxiety was associated with increased 
perceptions of relationship functioning and intrapersonal well-being for those recently reunited with their partners, relative to those not experiencing a reunion, but that these relationship perceptions decrease across the reunion. However, these findings suggest that at some point (here the 4.5 month mark), relationship perceptions might improve again.

Certainly, the number of curvilinear relationships between attachment dimensions and spousal outcomes as a function of time reunited were few, therefore, any conclusions should be interpreted with caution. With that in mind, conceptually, it may be the case that the more one is dependent on their partners for emotional comfort, reassurance, and validation, and the more one experiences increased distress in response to partner separations (so higher in attachment anxiety), the more one might respond positively to this honeymoon period. Indeed, those with greater needs for connection and reassurance in this initial reunion period might fare better because they might make more efforts to connect with their partners, and establish intimacy, or engage in more proximity-seeking and intimacy-building activities with their partners. However, the opposite might be true for those lower in attachment anxiety. For them, perceptions of relationship success and satisfaction was lower near the beginning of the reunion period, increased over time, and then began to decrease once again near the 4.5-month mark. It has been a fairly consistent pattern to find that the findings of those with lower levels of anxiety mirror those with higher levels of attachment avoidance. Individuals with less fear of rejection and abandonment, and those with greater discomfort with emotional intimacy might be engaging in less proximity-seeking behaviours, less of a desire for reconnection, and less initiation of, or enjoyment of, intimacy-building activities. 


\section{Mechanisms of Attachment Anxiety on Intrapersonal and Relationship Outcomes}

The final goal of Phase Three was to identify potential cognitive mechanisms that might explain the relationships between attachment anxiety and intrapersonal outcomes and relationship perceptions. As a result of a number of post-hoc mediation models (Preacher \& Hayes, 2008), I found that emotional fitness or the extent to which one maintains an optimistic outlook and is able to enjoy life, partially mediated the relationships between attachment anxiety and all coping outcomes including amount of daily psychological distress, subjective well-being, the ability to handle daily hassles, life mastery, coping success, one's ability to think critically in the face of stressors, and the use of self-blame and self-distraction as coping strategies. In addition, and in line with findings by Rholes and colleagues (2001), perceptions of partner support partially mediated the relationships between attachment anxiety and relationship satisfaction (two indices), perceived relationship success, and the extent to which one agrees with one's partner on a number of issues, and fully mediated the relationship between attachment anxiety and the extent to which partners engage in outside activities together.

\section{Conclusions}

In summary, the findings revealed in this final phase have corroborated and extended prior research in several ways. Consistent with previous research, I found that both attachment anxiety and attachment avoidance were relevant in explaining perceptions of intrapersonal coping and relationship functioning, particularly in the context of military experiences. I also found that attachment anxiety was related to emotion-focused coping strategies and decreased subjective well-being (on several indices), and attachment avoidance was associated with distancing coping strategies. However, extending prior research, attachment avoidance was associated with breakdowns in coping capacity and subjective well-being. Both attachment 
anxiety and avoidance were related to more negative relationship perceptions, consistent with previous research. Further, little research has reported interactions between anxiety and avoidance on intrapersonal coping. Extending prior research, I found that some of these negative effects of attachment anxiety on intrapersonal outcomes were exacerbated for those also low in avoidance, and most of the effects of attachment anxiety on relationship outcomes were exacerbated for those high in attachment avoidance.

Although the other two phases were assessed within a similar context across participants, this third phase of the project provided a broader view on the relationships among attachment dimensions and the impact of military experiences on relationship and interpersonal functioning with a larger sample than the first two phases and assessed couples at various phases of deployment-related experiences (preparing for a deployment, current separated, currently reunited, and neither separated nor recently reunited). Consistent with phases One and Two, I found that some of the effects of attachment orientations on perceptions of intrapersonal and interpersonal functioning were moderated by the presence or absence of an attachment-related threat (i.e., the deployment status of the partner) such as various stages of deployment separations and reunions. However, it is important to mention that many of the relationships among attachment dimensions and intrapersonal and relationship outcomes were not moderated by the deployment-related variables tested here. Indeed, once an alpha-rate correction was employed, many interactions among attachment dimensions and various deployment experiences were not statistically significant. With that in mind, with respect to deployment separations, my findings corroborate those of Egerton-Graham (2009) that the most challenging stage of the separation is stage 3 (weeks 3-6 of the separation) typically consisting of emotional disorganization. Indeed, whether or not spouses were experiencing this stage of a separation 
moderated many relationships among attachment dimensions and relationship outcomes specifically. In particular, lower levels of avoidance, and higher levels of anxiety, were associated with more positive relationship perceptions during this stage, while higher levels of avoidance, and lower levels of anxiety, were associated with more negative relationship perceptions during this stage.

Most importantly, because of this increased power relative to the other phases, I was also able to more easily assess the trajectory of the effects of attachment dimensions on intrapersonal and relationship outcomes as a function of time separated, and time reunited. With respect to the separation, there were not many effects of attachment avoidance or attachment anxiety as a linear function of time deployed on outcomes, but one finding consistent with those from Phase One, was that low levels of anxiety were associated with increases in perceived relationship success over time, whereas higher levels of anxiety were associated with decreases in perceived relationship success across the separation.

With respect to the reunion, I did not find much support for the notion that recent reunion status moderates relationships among attachment dimensions and spousal functioning. Regarding the findings that were significant, and in line with my predictions and the findings from Phase One, those with higher levels of attachment anxiety reported higher levels of relationship success, and the extent to which one agrees with their partner on various issues, for those recently reunited relative to those not recently reunited. While I did not find much support for the notion that attachment dimensions interact with months deployed in a linear fashion, I did find more support for curvilinear effects across time according to levels of attachment avoidance and anxiety and are worthy of highlighting for potential future research. In particular, for those with higher levels of attachment avoidance, perceptions of one's partner and the relationship 
were lower near the beginning of the reunion, increased over time, and then decreased after the 4.5-month mark (inverted-U shape). This makes sense, as having one's partner home would naturally increase levels of intimacy in the relationship making avoidant individuals uncomfortable and might potentially lead to conflict and more unpleasant partner interactions (Tidwell et al., 1996). However, at higher levels of attachment anxiety, perceptions of one's partner and the relationship were initially higher, decreased over time, and then started to increase again near the 4.5-month mark. It appears that the more one is dependent on their partners for emotional comfort, reassurance, and validation, and the more one experiences increased distress in response to partner separations, the more one responds positively to this honeymoon period. Indeed, those with greater needs for connection and reassurance (higher in attachment anxiety) in this initial reunion period are faring better because they might be making more efforts to connect with their partners, and establish intimacy, or are engaging in more proximity-seeking and intimacy-building activities with their partners.

Finally, with the plethora of measures included in the survey, and adequate sample size, I was able to use mediation models to identity consistent and powerful mediators in the relationships between attachment orientations and intrapersonal and relationship outcomes. Specifically, I found that the mediator that could best explain the relationships between attachment anxiety and the use of emotion-focused coping strategies, and decreased subjective well-being and increased psychological distress was decreased emotional fitness, or having a less optimistic outlook on life and less of an ability to enjoy life. Further, consistent with Rholes and colleagues (2001), perceptions of decreased partner support was the best explanation for the relationships between attachment anxiety and more negative relationship perceptions. 


\section{Chapter 6}

\section{General Discussion}

In summary, using different methodologies (longitudinal and cross-sectional), the most current measures of adult attachment that allows for more precise statistical and theoretical inferences, and three separate samples of Canadian spouses in married or common-law relationships, allowing me to test the robustness of any observed effects with replication, I was able to address the three major issues above. Specifically, I assessed 1) what adult attachment dimensions are relevant in explaining spousal reports of intrapersonal coping and perceptions of their partner and their relationship in various deployment-related contexts, 2) when such attachment dimensions are relevant in explaining spousal reports of intrapersonal and relationship functioning, and 3) how attachment dimensions explain reports of intrapersonal and relationship functioning across the separation and reunion (what trajectories) and finally 4) why attachment dimensions, particularly attachment anxiety, predicts spousal reports of intrapersonal and relationship functioning.

\section{Summary of Findings}

\section{Phase One}

I first examined a group of civilian spouses who were currently separated from their military partners, most of which had been deployed to Afghanistan. With this group currently experiencing a military deployment separation, higher levels of attachment anxiety were associated with reports of more compromised emotional well-being and more negative relationship perceptions relative to lower levels of attachment anxiety. This is consistent with prior research examining the relationship between anxious attachment and perceptions of emotional and relationship adjustment during attachment-related threats such as real or imagined 
partner separations, a deployment post-reunion period, and a stressful experience (Fraley \& Shaver, 1998; Cafferty et al., 1994; Collins \& Read, 1990). It would appear as though highly anxious individuals are acting in accordance with their internal working models. Specifically, just as anxious-resistant infants show strong separation protest to separation with their mothers, highly anxious adults show similar hyperactivating responses including emotional escalation, ineffective coping strategies in response to stress (emotion-focused strategies), and more negative relationship perceptions, relative to less anxious adults.

On the other hand, I found that attachment avoidance was associated with increased emotional well-being, but related to more negative relationship perceptions in this separated group. Highly avoidant individuals seem to be acting in accordance with their working models of attachment, effectively deactivating their attachment needs and engaging in distancing coping strategies (Simpson et al., 1992; Mayseless et al., 1996). Although some have suggested that breakdowns in the emotional well-being and coping capacity might be observed with increases in attachment avoidance with high enough levels of stress (Mikulincer \& Florian, 1998), this research suggests that the experience of a deployment separation overall is not a highly stressful event, at least when collapsing across all points of the separation.

One potential shortcoming of this study was the lack of a baseline, or control group. Specifically, it is understood that those who are insecurely attached typically experience less relationship satisfaction (Feeney, 2008; Rholes et al., 2001; Simpson, 1990), more negative affect and destructive communication patterns when interacting with their partners (Feeney, 2008; Simpson et al., 1996), than those who are securely attached. However, the fact that environmental variables moderated some of the relationships between attachment dimensions and relationship outcomes provides some evidence that stress moderates the effects of 
attachment dimensions, over and above their normal functioning. In other words, these findings are consistent with the notion that attachment orientations and attachment threats are interactive in nature (Campbell \& Marshall, 2010; Simpson \& Rholes, 1994). Indeed, extending prior research, I found that the effects of attachment anxiety were amplified depending on what point in the separation spouses were experiencing, and whether they were experiencing deployments for the first time. For example, I found that the effects of attachment dimensions on relationship perceptions were exacerbated for those experiencing their first deployment (Frankel, Snowden, \& Nelson, 1993; Padden, Connors, \& Agazio, 2011), and that perceptions of relationship quality continued to worsen in a linear fashion across the separation for those higher in attachment anxiety. Although no prior research has examined the effects of attachment anxiety during a prolonged partner separation, these findings do converge with those by Rholes and colleagues (2001) that found that perceptions of partner support continued to worsen for highly anxious women across the transition to parenthood for the first time.

Therefore, there are unique effects of both attachment anxiety and attachment avoidance on perceptions of emotional well-being and relationship functioning during a deployment separation, and the effects of both attachment dimensions seem to be somewhat chronic in nature (regardless of stage of the separation). However, I also found the effects of attachment anxiety on some perceptions of relationship quality and satisfaction were interactive in nature, exacerbated by certain environmental moderators (previous deployment experience and point in the separation).

I also examined a group of spouses who had been reunited with their military member partners, who for the most part had returned from an overseas deployment to Afghanistan (however a different group than the separation group). Initial research (Cafferty et al., 1994) 
examining the effects of adult attachment on intrapersonal and relationship outcomes following a military deployment reunion found that secure spouses reported less conflict and more satisfaction relative to insecure spouses, but there were no effects of spousal attachment styles on spousal emotional well-being. The findings from this study suggest that the effects of attachment anxiety specifically on outcomes during a reunion period extend beyond simply relationship perceptions. Specifically, I found that attachment anxiety was related to less effective emotional coping including decreased general well-being, less perceived social support, and more depressive symptoms. In addition, attachment anxiety was related to decreased relationship satisfaction, and feeling more strongly that the reunion has not lived up to one's original expectations. In addition, and extending previous findings, attachment avoidance was also associated with less relationship satisfaction during the post-reunion period.

Further extending prior work, and consistent with my separation findings, I found that the effects of attachment anxiety on some relationship outcomes were moderated by time reunited. For example, for highly anxious individuals their perceptions of their interactions with their partners declined as a function of time reunited. In addition, I was able to identify one potential mechanism to explain why higher levels of attachment anxiety are related to more negative relationship perceptions during the reunion period - beliefs that one's original expectations have not been met. Indeed, the relationships between attachment anxiety and decreased relationship satisfaction and quality of partner interactions were fully mediated by beliefs that the reunion period has not lived up to one's original expectations. This is consistent with the explanation proposed by Simpson and Rholes (2008) that the negative outcomes experienced by high anxiety individuals during the transition to parenthood reported by Rholes and colleagues (2001) might be tied to their unrealistically optimistic views of their romantic 
partners and relationships, and that highly anxious individuals may become frustrated and dissatisfied because they perceive that their partners are not living up to their expectations.

Finally, consistent with the airport findings of Fraley and Shaver (1998), higher levels of attachment avoidance were related to reports of less physical intimacy during the initial first meeting of the reunion relative to lower levels of avoidance. Again, this is more evidence that highly avoidant individuals avoid intimacy-building opportunities within their relationships, relative to those lower in avoidance (Tidwell et al., 1996).

\section{Phase Two}

Consistent with previous findings (Fraley \& Shaver, 1998; Collins \& Read, 1990) and in line with the findings from Phase One, attachment anxiety was related to a decreased ability to cope and to decreased relationship satisfaction during the ongoing deployment (across four months). However, I predicted that attachment anxiety would be related to difficulties in providing emotional support (Collins \& Feeney, 2000), and to greater fantasizing about the reunion (e.g., Davilla, 2001; \& Wei, Mallinckrodt, Larson, \& Zakalik, 2005), but neither of these predictions were supported. Although I predicted that attachment anxiety would be more related to perceptions that the reunion period has not lived up to one's original expectations in line with Phase One findings, and those reported by Rholes and colleagues (2001), this relationship was true but only for those with higher levels of attachment avoidance (those who are 'fearful').

In fact, the most notable findings for this group of spouses were regarding the effects of attachment avoidance. Specifically, in line with my predictions that attachment avoidance would be associated with deactivating strategies in response to environmental threats (e.g., Fraley \& Shaver, 1997), attachment avoidance was not related to indices of emotional well-being but was related to a decreased tendency to think and fantasize about one's partner and their return. 
Further, in line with findings by Collins and Read (1990), attachment avoidance was related to lower quality partner interactions, and difficulties with the provision of emotional support. Specifically, attachment avoidance was related to a general discomfort in expressing vulnerability to one's partner and not enjoying the process of providing emotional support to one's deployed partner. In addition, attachment avoidance was related to less satisfaction with the emotional support received from one's partner, and to pushing one's partner away when they do provide support.

With respect to the separated group's responses at the reunion, consistent with findings by Cafferty and colleagues (1994) and the Phase One reunion findings, attachment anxiety was related to decreased relationship satisfaction, but not to coping capacity. On the other hand, different from the reunion findings, attachment avoidance was now related to decreased coping capacity as assessed by all three indices (general well-being, perceived social support, and more depressive symptoms) suggesting a potential breakdown in coping abilities for this group of highly avoidant individuals as observed by Mikulincer and Florian (1998).

\section{Phase Three}

This phase was not only integrative and summative in nature, allowing me to replicate tests of hypotheses from earlier phases with a larger data set, but also allowed for a comprehensive view of the relationships among attachment dimensions and perceptions of current intrapersonal coping and relationship perceptions in spouses of Canadian military members. The number of variables that I had access to in this phase was extensive. This allowed me to more adequately assess the relevance of attachment dimensions, treated as stable and chronically accessible frameworks that guide one's ability to manage stressors (particularly those relevant to military spouses), and guide the strategies that individuals use to do so, 
regardless of environmental threats. However, I was also able to assess the alternative view that I have also been testing in previous phases, that attachment working models are interactive in nature and should mainly be expressed under conditions of threat (Campbell \& Marshall, 2010; Mikulincer et al., 2002), particularly under conditions that threaten the attachment bond such as deployments. The Phase Three survey contained several scales that tapped into indices of intrapersonal coping and well-being, but also several scales that tapped into perceptions of relationship functioning and quality. This allowed me to more effectively assess whether one view of the workings of attachment dimensions (chronic versus interactive) is more accurate for one type of outcome relative to another. In other words, I could assess whether the effects of attachment dimensions on perceptions of intrapersonal coping and perceptions of relationship functioning are more chronic in nature, or are more interactive with environmental threats. Finally, researchers have only recently started assessing attachment orientations with continuous measures, which allows for the simultaneous assessment of the unique and interactive effects of these constructs (attachment anxiety and attachment avoidance). This is an additional strength of this current line of research, that I can more effectively examine whether the effects of these dimensions on intrapersonal and relationship outcomes are unique or more interactive in nature. Further, this allows one to more accurately conclude whether it is truly attachment anxiety, attachment avoidance, or both, that can more adequately explain intrapersonal or relationship functioning and at what stages of a deployment. In addition, by treating both attachment anxiety and avoidance as continuous dimensions, one can more effectively assess nonlinear relationships between attachment dimensions and outcomes over time, and can also assess whether at any given point in time the effects of attachment dimensions on intrapersonal and relationship outcomes are nonlinear. 
First, I did find that attachment anxiety, attachment avoidance, and their interaction predicted responses to indices of emotional well-being and relationship functioning. In other words, regardless of context or the presence of environmental threats, attachment orientations explain perceptions of intrapersonal and relationship functioning. Specifically, attachment anxiety was associated with decreased emotional well-being and coping capacity (12/23 indices of well-being, coping capacity, and coping strategies), as well as more negative relationship perceptions (6/7 indices) in line with previous phases and previous work. Attachment avoidance was also associated with decreased emotional well-being (10/23), and more negative relationship perceptions (7/7 indices). These unique effects of attachment avoidance on indices of emotional well-being regardless of context are surprising given that such breakdowns in emotional wellbeing and coping capacity have only been observed in conditions of high stress (Mikulincer \& Florian, 1998). I also found that some of the negative intrapersonal coping outcomes observed for those higher in attachment anxiety relative to those lower in anxiety, were exacerbated for those also lower in attachment avoidance. Further, most of the negative relationship outcomes observed for those higher relative to lower in attachment anxiety were exacerbated for those also high in attachment avoidance. This latter finding is consistent with work by Collins and Feeney (2004) who found that fearfully attached individuals (high in attachment anxiety and avoidance) perceived their partners' personal messages of encouragement to be less supportive, controlling for the independent ratings of their partners' support in the messages. However, the interaction between anxiety and avoidance on coping outcomes have not previously been reported (Birnbaum, Orr, Mikulincer, \& Florian, 1997; Meyer, Olivier, \& Roth, 2005) and suggest that researchers should continue to examine potential interactions of the attachment dimensions in the future. 
To assess whether the effects of attachment dimensions on intrapersonal and relationship outcomes were also (and more or less so) interactive in nature, that is being most relevant in explaining responses to indices of intrapersonal coping and well-being and relationship quality under conditions of threat, I used the Canadian Deployment Handbook (Egerton-Graham, 2009) informed by work by Logan (1987) as a reference guide. This handbook very precisely has the stages of a deployment (including the deployment separation and reunion) identified that vary in the extent to which they are perceived to be stressful or emotionally 'reactive' in nature. Even more detailed, this handbook provides typical emotional responses that spouses can expect to feel during each stage, with the qualifier included that some spouses oscillate between stages or do not experience emotions typical in a particular stage (are even more or less reactive than the norm).

According to this handbook, the most challenging stage of the separation is stage 3 (weeks 3-6 of the separation) and consists of emotional disorganization (anxiety, emotional instability, clinginess, distrust, etc.). I assessed whether attachment dimensions interacted with the experience of this stage by comparing a group of individuals currently separated from their partners due to a deployment for one month to a group of spouses not currently separated or reunited with their partners due to a deployment. I did find that this variable, more than any other deployment-related variable, moderated relationships between attachment dimensions and relationship outcomes specifically, lending support to the notion that this period is a potentially threatening one. Surprisingly, those who perceive more relationship dysfunction during the highstress stage 3 relative to a baseline state (partner not deployed) are those with higher levels of attachment avoidance or greater discomfort with emotional closeness, and those with lower levels of attachment anxiety, or less fears of rejection and abandonment. Further, those with 
more positive perceptions of relationship functioning during this high-stress period are those with higher levels of attachment anxiety, or greater fears of rejection and abandonment, and those with low levels of attachment avoidance, or more comfort with emotional intimacy. This was in direct contradiction to the findings by Fraley and Shaver (1998), which showed that those who reported the greatest distress during an airport separation from a partner were anxiously attached women. This suggests that the trajectory of adjustment might not be linear and consistent for the same individuals. Indeed, some initial work on the relationship between attachment orientations and adjustment to life transitions suggests that perceptions of partner support for anxious attached individuals decline over time.

Following up on the logic above, I also tested both linear and curvilinear functions of time as a moderator of attachment avoidance, anxiety, and their interaction. There was little support for linear and curvilinear effects across time according to levels of attachment anxiety and avoidance, in that many findings did not meet my conservative threshold for statistical significance. However, the findings that were significant tended to be in line with those from Phase One, that low levels of anxiety were associated with increases in perceived relationship success over time, whereas higher levels of anxiety were associated with decreases in perceived relationship success across the separation. Further, given that the nature of emotional reactivity across the separation is curvilinear according to the Canadian Forces Deployment Handbook (Egerton-Graham, 2009), it wouldn't be surprising to see some curvilinear effects of outcomes according to attachment dimensions, across time separated and reunited. Specifically, spouses progress from more emotional 'reacting' stages (Stages 1-3) to emotional stabilization (Stages 45), and then into another reacting stage, Stage 1 of their reunion, which is the anticipation of their partner's homecoming. There were few curvilinear effects of attachment dimensions as a 
function of time separated. One finding was that for those higher in attachment anxiety (the same conceptual 'group' as those in the Rholes and colleagues 2001 study) perceived relationship satisfaction assumed an inverted-U shape, being less positive near the beginning and end of the separation reports, but more positive near the middle of the separation. Taken together, a group that showed variability in relationship perceptions across the separation were those with higher levels of anxiety. These findings are very much in line with work showing that individuals higher in attachment anxiety do tend to be more variable in their responses to relationship conflicts (Campbell, Simpson, Boldry, \& Kashy, 2005; MacDonald, Wood, and Fabrigar, submitted for publication; Tidwell, Reis, \& Shaver, 1996). It appears that these individuals are also those more variable in their responses to deployment separations.

With respect to the reunion, I did not find much support for the notion that recent reunion status moderates relationships among attachment dimensions and spousal functioning. Regarding the findings that were significant, and in line with my predictions and the findings from Phase One, those with higher levels of attachment anxiety reported higher levels of relationship success, and the extent to which one agrees with their partner on various issues, for those recently reunited relative to those not recently reunited. While I did not find much support for the notion that attachment dimensions interact with months deployed in a linear fashion, I did find more support for curvilinear effects across time according to levels of attachment avoidance and anxiety. In particular, for those with higher levels of attachment avoidance, perceptions of one's partner and the relationship were lower near the beginning of the reunion, increased over time, and then decreased after the 4.5-month mark (inverted-U shape). This makes sense, as having one's partner home would naturally increase levels of intimacy in the relationship making avoidant individuals uncomfortable and might potentially lead to conflict and more 
unpleasant partner interactions (Tidwell et al., 1996). However, at higher levels of attachment anxiety, perceptions of one's partner and the relationship were initially higher, decreased over time, and then started to increase again near the 4.5-month mark. It appears that the more one is dependent on their partners for emotional comfort, reassurance, and validation, and the more one experiences increased distress in response to partner separations, the more one responds positively to this honeymoon period. Indeed, those with greater needs for connection and reassurance (higher in attachment anxiety) in this initial reunion period are faring better because they might be making more efforts to connect with their partners, and establish intimacy, or are engaging in more proximity-seeking and intimacy-building activities with their partners.

It is common in the attachment literature to assume that the effects of attachment dimensions are linear in nature. However, it is important for researchers to start to test these assumptions that we so consistently rely on, so that our theoretical contributions are accurate and informed. Therefore, I wanted to test for the potential non-linear effects of attachment dimensions in the context of military deployments. There were no statistically significant (at least that met my conservative alpha criteria) curvilinear effects of attachment dimensions on indices of well-being as a function of low and high points of months deployed. However, I do encourage researchers to continue to assess potential nonlinear effects of attachment dimensions on intrapersonal and relationship outcomes, as there may be particular contexts where such interactions are valid and should be acknowledged.

Finally, it is not clear from the current literature on attachment and long term separations and major life transition periods why particular attachment dimensions are related to such outcomes, or what mechanisms can explain these relationships. As a result of a number of posthoc mediation models (Preacher \& Hayes, 2008), I found that emotional fitness or the extent to 
which one maintains an optimistic outlook and is able to enjoy life, partially mediated the relationships between attachment anxiety and all coping outcomes. In addition, and in line with findings by Rholes and colleagues (2001), perceptions of partner support partially mediated the relationships between attachment anxiety and most relationship outcomes, and fully mediated the relationship between attachment anxiety and the extent to which partners engage in outside activities together.

In summary, the findings from this phase are extensive, corroborating and contributing to our awareness of attachment processes during major life transitions, specifically military deployments in several ways. First, attachment anxiety and avoidance have unique and interactive relationships with indices of intrapersonal coping and relationship functioning. In addition, the attachment anxiety dimension seemed more reactive with environmental threats such that those higher in attachment anxiety seemed the most variable in their responses and adjustment to military deployment. Interestingly, these individuals showed better relationship perceptions during periods typically described as more 'reactive' and stressful, but showed declines in relationship perceptions during periods that are typically associated with recovery and stabilization. Further, there were similarities in the ways that individuals with higher levels of anxiety and those lower in avoidance responded to deployment experiences, and the ways that those lower in anxiety and those higher in avoidance responded to these same experiences which might be of theoretical importance in the future. Finally, I identified two major mechanisms that explained variation in perceptions of intrapersonal coping and relationship functioning according to levels of attachment anxiety, namely emotional fitness and perceived partner support, respectively. 


\section{Limitations}

Although there were several consistencies across phases with respect to which attachment dimensions seemed most relevant, the areas with which they were relevant, and whether the effects of attachment dimensions should best be considered chronic or more interactive with environmental stressors, these findings should of course be qualified by some important limitations.

Perhaps most importantly, these data are exclusively self-report. In other words, the spouses are reporting about their perceptions of their relationship and intrapersonal adjustment which may or may not accurately reflect their true state of adjustment or relationship quality. In fact, it is characteristic for those higher in attachment anxiety to amplify their signals and experiences of distress perhaps to elicit care from their attachment figures. On the other hand, individuals higher in attachment avoidance characteristically under report their experiences of distress, but perhaps overstate their negative relationship perceptions, in efforts to deactivate experiences of distress and avoid rejection from an attachment figure. Therefore, it is possible that individuals higher in attachment anxiety are faring better than they are reporting, with respect to actual coping and relationship experiences, and that those higher in attachment avoidance are faring much worse than they are projecting.

To put this view into perspective, an abundance of research has shown that secondary or insecure attachment styles are associated with perceptions of one's ability to regulate emotions, one's coping capacity, and stress management, and that these strategies can place a person at an increased risk for psychopathology. In other words, rather than being limited to simply perceptions of one's ability to manage threats (particularly related to the relationship), insecure attachment styles can place an individual at risk for more several psychological and health 
disturbances. Clinicians have observed that those who engage in hyperactivating attachment strategies (higher in attachment anxiety) have higher levels of clinical diagnoses of global distress, depression, anxiety, eating disorders, and substance abuse (see Mikulincer \& Shaver, 2008). On the other hand, while deactivating strategies might be associated with successful suppression of rejection-related thoughts and behavioural distancing towards a romantic partner, other research suggests that the deactivating form of a secondary attachment strategy might place an individual at risk for more serious psychological and physiological health diagnoses including mood disorders and somatic complaints (see Mikulincer \& Shaver, 2008). However, the list of more severe psychological or health disturbances for those with more anxious relative to avoidant attachment orientations is more extensive. In other words, those who tend to show greater subjective distress and heightened reactions to attachment-related threats do tend to be more at risk for more severe psychological and health disturbances relative to those who show emotional and behavioural distancing in response to attachment-related threats. Further, selfreported attachment dimensions are correlated with observer's reports of communication styles and hostility during conflict with romantic partners (Kobak \& Hazan, 1991), behavioural displays of affection and avoiding contact with a partner during an interaction (Feeney, 1998), and attachment orientations are related to relationship outcomes like its stability over time (Kirkpatrick \& Davis, 1994; Kirkpatrick \& Hazan, 1994). Therefore, the predictive validity of attachment orientations to relationship outcomes and more objective measures of relationship behaviour is well established.

One of the major limitations of Phases One and Two were the sample sizes. Therefore, I was limited in the extent to which I could generalize such findings to the larger population of Canadian married couples in married or common-law relationships with a CAF member, and test 
more complex statistical analyses. Further, both samples were homogenous in nature, capturing spouses going through similar military experiences and at a similar point in time. This made it challenging for me to assess linear or curvilinear effects of time given the restricted ranges of time separated or reunited, and to generalize to beyond the experiences of this particular sample. In addition, little research has assessed how attachment dimensions are predictive of subjective accounts of intrapersonal and relationship functioning during military separations and reunions, so there was a not a lot of literature that I could compare my results against and confidently challenge or support a theoretical framework of attachment processes during military deployments. Fortunately, however, Phase Three was well powered and allowed me to more effectively observe consistencies and inconsistencies in findings across all phases. Phase Three contained a more heterogenous sample comprised of civilian and military spouses of military members in the RCN, CA, and the RCAF. More importantly, this survey captured spouses at all stages of deployments and separations, including those not currently separated nor recently reunited. Related to this point, one might question the extent to which findings from Phases One and Two are truly an effect of deployment separations and reunions, or whether we might find similar patterns of relationships between attachment dimensions and reports of intrapersonal and relationship functioning in those not currently reunited or separated from their partners. In other words, to what extent are the findings observed in Phases One and Two limited to separations and reunions, or do they extend to more mundane relationship experiences? Again, Phase Three allowed me to compare responses of those currently experiencing the most reportedly stressful separation and reunited stages against baseline groups, or those not currently preparing for, nor experiencing a separation or a recent reunion with their partners. Indeed, there were some important differences between those experiencing stressful separation/reunion periods and 
baseline groups in outcomes per attachment dimensions. Therefore, this final Phase Three allowed me to more accurately assess when attachment dimensions are predictive of outcomes, and in what way.

In addition, this research was exclusively focused on the perceptions of the spouse. For this reason, it is difficult to determine to what extent intrapersonal and relationship perceptions are driven by internalized working models, and to what extent reports of spousal functioning are influenced by partner behaviour. Theoretically, and demonstrated empirically, internal working models tend to be fairly stable across time and relationships (Baldwin \& Fehr 1995; Scharfe \& Bartholomew, 1994; Waters et al., 2000). While it is possible that partner behaviour can influence both attachment orientations and intrapersonal and relationship functioning, partner behaviour more realistically might be adding error variance, or variance in intrapersonal and relationship outcomes that attachment orientations therefore cannot account for. Some researchers in the field of adult attachment have assessed and controlled for partner effects in their investigations of relationships among attachment orientations and relationship perceptions and behaviours and found that the relationships between attachment dimensions and relationship outcomes do not fluctuate much (significant findings remain significant) once partner behaviour is considered (Feeney, 1998; Rholes et al., 2001). However, theoretically it is important to continue to assess the role of partner behaviour in various attachment-related relationship processes, and how partner behaviour interacts with attachment orientations in explaining relationship behaviours and perceptions of functioning and quality.

Lastly, while the response rate across all phases might seem low (ranging from relative to $21-23 \%$ ) these rates are typical for military samples that have hovered around 30\% compliance since the 1980s (e.g., Lamerson, 2001; Newell, Rosenfeld, Harris, \& Hindelang, 2004). These 
rates are also consistent with results of other studies using home address mailings to contact married couples (e.g., 16.7\% in Banse, 2004; 17.8\% in Davila, Karney, \& Bradbury, 1999; 18\% in Kurdek, 1991). Further, other studies of married individuals previously discussed relied mainly on the use of convenience sampling such as recruiting through advertisements or community centres and therefore cannot accurately assess nor report response rates (e.g., Birnbaum et al., 1997; Lussier et al., 1997; Meltzer et al., 2014; Neff \& Geers, 2013; Rholes et al., 2001). However consistent with previous research, our response rate indicates that our findings may not be representative of the typical married/common-law population, or the typical military spousal population. With this mind, it is important to consider that the main focus of this research was to assess relationships among variables of interest. Although it is possible that limitations in sampling methods, size, and characteristics might impact the findings in a way that range of responses are restricted, inflated, or constants added (the typical response is lower or higher than typical), it is less likely for such sampling issues to change the nature of relationships among variables from what is expected theoretically. In other words, I do not expect such sampling issues to significantly impact the nature of relationships among attachment orientations and indices of intrapersonal and relationship functioning.

\section{Contribution to Previous Literature}

Past literature on the relevance and nature of effects of attachment dimensions on spousal functioning during military deployments is fairly limited in amount, scope, measurement of adult attachment, and complexity of statistical analyses. These findings contribute to this literature in a number of ways. Although the literature on affect regulation and coping with stress is fairly extensive and does find that anxious and avoidant attachment styles predict different methods of affect regulation and coping strategies and success in responses to stressors, I found 
fairly consistent evidence for the interactive effects of anxiety and avoidance on perceptions of intrapersonal coping and relationship outcomes. Specifically, I found that some of the negative effects of attachment anxiety on intrapersonal outcomes were exacerbated for those also low in avoidance, and most of the effects of attachment anxiety on relationship outcomes were exacerbated for those high in attachment avoidance. Further, most of the literature assessing attachment styles and coping outcomes finds less evidence for a positive relationship between attachment avoidance and breakdowns in coping capacity and subjective well-being, and when researchers have reported such breakdowns, they have been limited to very stressful experiences (Mikulincer \& Florian, 1998). Contrary to these findings, in my more extensive (in sample and measures) Phase Three study, I found that attachment avoidance was related to a number of indices capturing coping capacity and subjective well-being. Although this might in part be explained by differences in the individuals sampled (military spouses relative to civilian spouses in the previous literature), what might also explain this inconsistency is the use of typological versus continuous measures of attachment orientations.

The bulk of research examining relationships among attachment orientations and coping outcomes has used the typological approach (e.g., Birnbaum et al., 1997; Lussier et al., 1997; Mikulincer et al., 1993). Using the continuous approach, I was able to simultaneously consider the unique and interactive effects of attachment anxiety and attachment avoidance on indices of intrapersonal functioning and perceptions of relationship functioning in my statistical models. It is possible that the relationships between attachment avoidance and coping outcomes can more effectively be observed once one accounts for the unique effects of attachment anxiety, and their interaction terms together. Therefore, in questioning what attachment dimensions are relevant in explaining differences in coping and relationship perceptions overall (regardless of deployment 
status), it appears that attachment anxiety, attachment avoidance, and their interaction are important predictors.

In terms of what attachment dimensions are relevant at various deployment stages, both attachment anxiety and attachment avoidance seem to be relevant in explaining decreased coping and more negative relationship perceptions during deployment separations, avoidance may uniquely predict difficulties with provision and acceptance of emotional support during the ongoing separation, and anxiety seems to better predict outcomes during deployment reunions (Phases One and Three). The only study assessing relationships among spousal attachment orientations and adjustment following a military reunion (Cafferty et al., 1994) found that an anxious attachment was related to perceptions of relationship functioning, but not indices of emotional well-being. Phases One, Two, and Three found evidence that anxiety was predictive of more than just relationship outcomes, that attachment anxiety also predicted decreased emotional adjustment and coping capacity outcomes. Further, in Phase One I demonstrated that perceptions that the reunion period has not lived up to one's expectations fully mediated the relationships among attachment anxiety and relationship outcomes (just not indices of emotional adjustment). Although this is in line with theorizing by Simpson and Rholes (2008), this is a novel finding. Finally, as previously mentioned, attachment avoidance was related to more negative perceptions about one's provision of and acceptance of emotional support with one's partner, and less fantasizing and thinking about one's partner during a deployment separation. Although these findings might be consistent with a larger literature on attachment avoidance and support provision/acceptance and attachment-related thoughts, these findings have not been extended to the more serious context of an ongoing deployment-related separation. 
As outlined by Simpson and Rholes (1994) and further reiterated by Campbell and Marshall (2010), attachment dimensions are interactive in nature and are therefore most relevant in contexts signalling attachment concerns. In situations that threaten the attachment bond, including threats to the well-being of either the individual or attachment figure, we should see internal working models and attachment strategies most salient, pronounced, and expressed. In other words, attachment dimensions should be most predictive of attachment-related cognitions, emotions, and behaviours under threatening conditions relative to neutral conditions. I found some evidence to support this notion, that there are some deployment-related events that moderate the effects of attachment dimensions on intrapersonal and relationship outcomes. In other words, I was able to further determine when attachment dimensions are most relevant across the deployment cycle.

First, the negative effects of deployment separations seem to be amplified for those who are experiencing their first deployment (Frankel, Snowden, \& Nelson, 1993; Padden, Connors, \& Agazio, 2011). I further found that the relationship between attachment anxiety and more negative relationship perceptions during a deployment separation were amplified for those experiencing their first deployment. In fact, after the first deployment the relationship between attachment anxiety and relationship perceptions was no longer significant. These findings suggest that attachment anxiety, in particular, might be most relevant in predicting relationship outcomes in contexts that one has less experience and cannot adjust their expectations.

I also found that, across the three phases, the dependent measures that appeared to be less related to, or interactive with, deployment-related contexts were reports of emotional wellbeing and coping capacity, while reports of relationship satisfaction and quality were more dependent on, or interactive with, deployment-related contexts. Specifically, one deployment 
context that was particularly interactive with attachment dimensions in predicting relationship outcomes was whether or not spouses were recently separated $(\leq 1$ month) from their romantic partners. Some of these findings were consistent for Phases One and Three. Specifically, those lower in attachment avoidance or those higher in attachment anxiety had more positive relationship perceptions during this time, relative to those not separated, however those higher in avoidance had more negative relationship perceptions for those also recently separated relative to those not separated. I speculate that those with greater needs for connection and reassurance (lower avoidance or higher anxiety) in this initial period are faring better because they might be making more efforts to connect with their partners, and establish intimacy, or are engaging in more proximity-seeking and intimacy-building activities with their partners. This might be reflected in increased attempts to contact one's partner, more intimate conversations involving support seeking and provision, and successfully gaining that reassurance they so desperately desire. It is also possible that their partners are more accepting of their partners' 'neediness' during this stage, relative to those at home together, and so highly anxious, or less avoidant, individuals are receiving the attention they desire normally but perhaps do not receive to the same extent. However, those with greater levels of discomfort with emotional intimacy and dependency, or less fears of rejection and abandonment are less motivated to connect with their partners, and maintain intimacy, from afar. In other words, tendencies to engage in de-activating strategies to reduce psychological dependency on one's partner, or less of a concern about rejection and abandonment, might lead these individuals to not reach out to one's partner as often or as intimately, to not provide nor seek as much emotional support from one's partner, and more of a 'out of sight out of mind' mentality. 
In addition, my research provides some clarification surrounding how attachment dimensions predict intrapersonal and relationship functioning during deployment stages. In other words, I found evidence to suggest that the trajectories of intrapersonal and relationship outcomes across deployment separations and reunions differ according to levels of attachment anxiety and attachment avoidance, both in linear and nonlinear fashions. However, I need to preface these findings by mentioning that the number of interactions among attachment dimensions and deployment-related moderators were few after making an alpha criterion correction, and therefore any interpretations should be made caution. However, these findings are worthy of some attention, as they might be able to point to interesting avenues for future research, if they are replicated.

One consistent pattern across Phases One and Three was that relationship perceptions for those higher in attachment anxiety became more negative (so linear) across the separation and reunion periods. Indeed, attachment anxiety was related to decreases in relationship satisfaction and harmony across the separation in Phase One, and decreased relationship success across the separation in Phase Three. In addition, I found some evidence to suggest that some of the relationships among attachment dimensions and relationship and coping outcomes might vary in a curvilinear nature across the separation and reunion periods. However, because I could only adequately test these functions in Phase Three, and there were too few effects found, further research in this area is needed before any conclusions can should be drawn. Finally, the current literature portrays those with anxious attachments as most distressed by long term separations (e.g., Fraley \& Shaver, 1998; Mayseless et al., 1996), however this research suggests that this is too general an understanding, and that there are points in a long-term separation where highly 
anxious individuals could potentially be functioning better than lower anxiety individuals, or at least better than they typically do in relationships (i.e., not separated).

Finally, I was able to more adequately identify why attachment dimensions are related to intrapersonal coping and relationship outcomes which at this point is not well understood. In Phase Three, I conducted a number of mediation models to determine which indices most effectively explained relationships between attachment dimensions and intrapersonal and relationship outcomes. My findings suggest that the best mediator to explain the relationships between attachment anxiety and the use of emotion-focused coping strategies, and decreased subjective well-being and increased psychological distress is decreased emotional fitness, or having a less optimistic outlook on life and less of an ability to enjoy life. Further, and consistent with Rholes and colleagues (2001), perceptions of decreased partner support was the best explanation for the relationships between attachment anxiety and more negative relationship perceptions. This is a novel contribution to the understanding of the relationships among attachment dimensions and intrapersonal and relationship functioning during military deployments. These findings suggest that fluctuations in perceptions of subjective well-being and relationship quality during long term separations and reunions are functions of fluctuations in optimism and the extent to which you feel your partner is supportive of you and the relationship.

\section{Implications and Future Directions}

This research has the potential to make significant contributions to current governmental programs, policies, and services that support the quality of life of CAF families such as the mandated Family Separation and Reunion service provided by Military Family Resource Centres (MFRCs). The Road to Mental Readiness (R2MR) is a program provided to CAF members and 
their families with the goal of improving soldiers' short term performance and long term mental health outcomes (Department of National Defence, 2015). Part of this training involves preparing CAF members and their families for military deployments, with guidelines and information based on research internal and external to the Department of National Defence. This program helps spouses in particular to understand the phases of a deployment and typical thoughts, behaviours, and emotions experienced during each of these stages. Although the current R2MR does acknowledge variation amongst spouses in their functioning during each of the stages, and the progression or oscillation between stages, this research could help provide more accurate guidelines with adult attachment as the theoretical framework. Specifically, this research could help explain some of that variance, and reduce some of this ambiguity by helping spouses to identify their attachment orientations and then provide them with more accurate trajectories and expected outcomes as a function of such orientations. By identifying and adding predictors of spousal adjustment to such programs, we can help to maximize the extent to which spouses can be effectively assisted and supported, and ensure that governmental resources such as the R2MR are most effectively utilized (see Canadian Forces Moral and Welfare Services for information on current programs for CAF personnel and their families).

For example, aside from being able to identify who might be most at risk for experiencing difficulties in intrapersonal and relationship functioning during particular phases of the deployment cycle, programs could also be aimed at also targeting identified mechanisms or mediators of intrapersonal and relationship functioning. This research suggests that having unrealistically optimistic expectations of a partner's return might be problematic when a partner's return does not live up to one's original expectations, and that those higher in attachment anxiety are most likely to report such beliefs. In addition, emotional fitness might 
well explain relationships among attachment anxiety and coping outcomes. Future interventions could be developed whereby spouses could be randomly assigned to receive the current spousal support programs for deployment preparation, and another group of spouses randomly assigned to a program with a module specifically designed to address problems associated with unrealistic expectations of the reunion, and to develop expectations that are more realistic, and a more optimistic outlook. This would allow researchers and agencies to assess the effectiveness of expectation modification in spousal adjustment and relationship functioning in couples experiencing a deployment, and lead to the development of optimally effective spousal support programs.

\section{Conclusion}

John Bowlby, the founder of attachment theory, created one of the most complete theoretical accounts of how infants react to the experience of separation from an attachment figure (Bowlby, 1973), how this experience is moderated by internal working models of attachment figure responsiveness and availability, and how repeated experiences of separations impact an infant's development. Bowlby also speculated that attachments are 'from the cradle to the grave', that our attachment models follow us through life and impact later relationships with important others, which lead to the development of adult attachment theory. To this day, attachment researchers are still gaining an understanding of how attachment processes operate in adulthood. John Bowlby (1973) and his graduate student Mary Ainsworth (1978) demonstrated how separations and reunions with attachment figures are contexts within which attachment processes are activated and therefore most pronounced. The military deployment contains elements comparable to Mary Ainsworth's Strange Situation used to classify infant attachment styles, involving separations from an attachment figure, introduction of a potential threat (stranger for 
infants, injury for adults), and a reunion. In other words, a military deployment is akin to the adult version of the Strange Situation.

Unfortunately, little research has assessed the relevance of attachment orientations in explaining spousal adjustment and relationship functioning in this context. Although one initial study points to the relevance of adult attachment in spousal adjustment to the post-reunion period (Cafferty et al., 1994), methodological concerns and lack of more complex statistical analyses leave many questions unanswered. This three-phased research project was able to clarify what attachment dimensions specifically are related to problems in emotional and relationship adjustment during deployment stages, when attachment dimensions are relevant in predicting outcomes across the deployment cycle, how attachment dimensions predict intrapersonal and relationship functioning during deployment stages (what the trajectories of outcomes look like across time as moderated by attachment dimensions), and finally I was able to more specifically understand why attachment dimensions are related to intrapersonal coping and relationship outcomes which at this point is not well understood. As Bowlby (1973) so eloquently articulated, while it is not easy to understand why some individuals should recover from experiences of separation while others do not, we are starting to gain a better understanding thanks to the theoretical framework that is attachment theory. 


\section{References}

Aiken, L. S., \& West, S. G. (1991). Multiple regression: Testing and interpreting interactions. Newbury Park, CA: Sage.

Ainspan, C.J. Bryan, \& W.E. Penk (Eds.). Handbook of Psychosocial Interventions for Veterans: A Guide for the Non-Military Mental Health Clinician. New York, NY: Oxford University Press.

Ainsworth, M. S., Blehar, M. C., Waters, E., \& Wall, S. (1978). Patterns of attachment: A psychological study of the strange situation. Oxford, England: Lawrence Erlbaum.

Bartholomew, K., \& Horowitz, L. M. (1991). Attachment styles among young adults: A test of a four-category model. Journal of Personality and Social Psychology, 61(2), 226-244.

Baldwin, M. W., \& Fehr, B. (1995). On the instability of attachment style ratings. Personal Relationships, 2, 247-261.

Baldwin, M. W., \& Kay, A. C. (2003). Adult attachment and the inhibition of rejection. Journal of Social and Clinical Psychology, 22(3), 275-293.

Banse, R. (2004). Adult attachment and marital satisfaction: Evidence for dyadic configuration effects. Journal or Social and Personal Relationships, 21(2), 273-282.

Baumeister, R. F. (1998). The self. In D. T. Gilbert, S. T. Fiske, \& G. Lindzey (Eds.), Handbook of social psychology (4th ed., pp. 680-740). New York, NY: McGraw-Hill.

Belsky, J., Rovine, M., \& Taylor, D. G. (1984). The Pennsylvania infant and family development project: III. The origins of individual differences in infant-mother attachment: Maternal and infant contributions. Child Development, 55(3), 718-728. 
Birnbaum, G. E., Orr, I., Mikulincer, M., \& Florian, V. (1997). When marriage breaks up: Does attachment style contribute to coping and mental health? Journal of Social and Personal Relationships, 14, 643-654.

Bowen, G. L., \& Martin, J. A. (2011). Support and Resiliency Inventory (SRI) for civilian spouses of U.S. Air Force members (active duty only): Descriptive summary (October 1, 2009 to December 31, 2010). Charlotte, NC: Flying Bridge Technologies, Inc.

Bowlby, J., (1969, 1982). Attachment and loss: Vol.1. Attachment. New York: Basic Books. Bowlby, J. (1973). Attachment and loss: Vol. 2: Separation: Anxiety and anger. New York: Basic Books.

Burant, E., Mikulincer, M., \& Shaver, P. R. (2008). Mothers' attachment style, their mental health, and their children's emotional vulnerabilities: A 7-year study of children with congenital heart disease. Journal of Personality, 76(1), 31-66.

Busby, D. M., Christensen, C., Crane, D. R., \& Larson, J. H. (1995). A revision of the dyadic adjustment scale for use with distressed and nondistressed couples: Construct hierarchy and multidimensional scales. Journal of Marital and Family Therapy, 21(3), 289-308.

Canadian Army, Government of Canada. (2017). Reserve Jobs. Retrieved from http://www.army-armee.forces.gc.ca/en/jobs-careers/reserve.page

Canadian Forces Moral and Welfare Services. (nd). Programs and Services. Retrieved from https://www.cafconnection.ca/National/Programs-Services.aspx.

Cafferty, T. P., Davis, K. E., Medway, F. J., O’Hearn, R. E., \& Chappell, K. D. (1994). Reunion dynamics among couples separated during Operation Desert Storm: An attachment theory analysis. In K. Bartholomew \& D. Perlman (Eds.), Advances in Personal Relationships: Attachment Processes in Adulthood (Vol. 5, pp. 309-330). London, UK: Jessica Kingsley. 
Campbell, L., \& Marshall, T. (2011). Anxious attachment and relationship processes: An interactionist perspective. Journal of Personality, 79, 917-947.

Campbell, L., Simpson, J.A., Boldry, J.G., \& Kashy, D. (2005). Perceptions of conflict and support in romantic relationships: The role of attachment anxiety. Journal of Personality and Social Psychology, 88, 510-531.

Carver, C. S. (1997). You want to measure coping but your protocol's too long: Consider the Brief COPE. International Journal of Behavioral Medicine, 4, 92-100.

Carver, C. S. (2007). Brief COPE. Retrieved from http://www.psy.miami.edu/faculty/ccarver/sclBrCOPE.html.

Cassidy, J. (2008). The nature of the child's ties. In J. Cassidy \& P. R. Shaver (Eds.), Handbook of attachment: Theory, research, and clinical applications (pp. 3- 20). New York: Guilford Press.

Chandra, A., Burns, R. M., Tanielian, T., Jaycock, L. H., \& Scott, M. (2008). Understanding the impact of deployment on children and families - findings from a pilot study of operation purple camp participants. Rand Working Paper Prepared for the National Military Family Association.

Cobeanu, O. (2013). Irrational beliefs and somatosensory amplification in breast cancer patients undergoing treatment: impact on general distress. Journal of Cognitive and Behavioral Psychotherapies, 13, 547-556.

.Cohen, J. (1960) A coefficient of agreement for nominal scales. Educational and Psychological Measurement 20, 37-46. 
Collins, N. L., \& Feeney, B. C. (1991). Working models of attachment shape perceptions of social support. Evidence from experimental and observational studies. Journal of Personality and Social Psychology, 87(3), 363-383.

Collins, N. L., \& Read, S. J. (1990). Adult attachment, working models, and relationship quality in dating couples. Journal of Personality and Social Psychology, 58(4), 644-663.

Davila, J. (2001). Refining the association between excessive reassurance seeking and depressive symptoms: The role of related interpersonal constructs. Journal of Social and Clinical Psychology, 20,538-559.

Davila, J., Karney, B. R., \& Bradbury, T. N. (1999). Attachment change processes in the early years of marriage. Journal of Personality and Social Psychology, 76(5), 783-802.

Davis, D., Shaver, P. R., \& Vernon, M. L. (2003). Physical, emotional, and behavioral reactions to breaking up: The roles of gender, age, emotional involvement, and attachment style. Personality and Social Psychology Bulletin, 29(7), 871-884.

De Burgh, T. H., White, C. J., Fear, N. T., \& Iverson, A. C. (2011). The impact of development to Iraq or Afghanistan on partners and wives of Military personnel. International Review of Psychiatry, 23(2), 192-200.

De Wolff, M.S., \& van Ijzendoorn, M.H. (1997). Sensitivity and attachment: A meta-analysis on parental antecedents of infant attachment. Child Development, 68, 571-591.

Department of National Defence. (2015). Road to Mental Readiness (R2MR). Retrieved from http://www.forces.gc.ca/en/caf-community-health-services-r2mr/index.page.

Diener, E., Emmons, R. A., Larsen, R. J., \& Griffin, S. (1985). The satisfaction with life scale. Journal of Personality Assessment, 49(1), 71-75. 
Duemmler, S. L., \& Kobak, R. (2001). The development of commitment and attachment in dating relationships: attachment security as relationship construct. Journal of Adolescence, 24(3), 401-415.

Efron, B., \& Tibshirani, R. J. (1993): An introduction to the bootstrap. Chapman \& Hall. Egerton-Graham, M. (2009). A practical guide for families. Canadian Forces Reunion Handbook. Retrieved from https://www.cafconnection.ca/getmedia/ed11832b-b5d54ea0-ba60-6a6e3d8e16b9/Reunion-Handbook.aspx.

Enders, C. K., \& Tofighi, D. (2007). Centering predictor variables in cross-sectional multilevel models: a new look at an old issue. Psychological Methods, 12(2), 121-138.

Fabrigar, L. R. \& Wegener, D. T. (2012). Exploratory Factor Analysis. New York: NY, Oxford University Press.

Fabrigar, L. R., Wegener, D. T., MacCallum, R. C., \& Strahan, E. J. (1999). Evaluating the use of exploratory factor analysis in psychological research. Psychological Methods, 4(3), 272-299.

Feeney, J. A. (1998). Adult romantic attachment: Developments in the study of couple relationships. In J. Cassidy \& P. R. Shaver (Eds,). Handbook of attachment: Theory, research, and clinical applications (pp. 456-481). New York: Guilford Press.

Feeney, J. A., \& Noller, P. (1992). Attachment style and romantic love: Relationship dissolution. Australian Journal of Psychology, 44(2), 69-74.

Fraley, L. R. (2002). Attachment stability from infancy to adulthood. Journal of Personality and Social Psychology Review, 6(2), 123-151. 
Fraley, L. R. (2010). Information on the Experiences in Close Relationships-Revised (ECR-R) Adult Attachment Questionnaire. Retrieved from http://internal.psychology.illinois.edu/ rcfraley/measures/ecrr-oldernorms.htm.

Fraley, R. C., \& Bonanno, G. A. (2004). Attachment and loss: A test of three competing models on the association between attachment-related avoidance and adaptation to bereavement. Personality and Social Psychology Bulletin, 30, 878-890.

Fraley, R. C., Fazzari, D. A., Bonanno, G. A., \& Dekel, S. (2006). Attachment and psychological adaptation in high exposure survivors of September $11^{\text {th }}$ attach on the World Trade Center. Personality and Social Psychology Bulletin, 32, 538-551.

Fraley, R. C., \& Shaver, P. R. (1997). Adult attachment and the suppression of unwanted thoughts. Journal of Personality and Social Psychology, 73, 1080-1091.

Fraley, R. C., \& Shaver, P. R. (1998). Airport separations: A naturalistic study of adult attachment dynamics in separating couples. Journal of Personality and Social Psychology, 75(5), 1198-1212.

Fraley, R. C., \& Waller, N. G. (1998). Adult attachment patterns: A test of the typological model. In J. A. Simpson \& W. S. Rholes (Eds.), Attachment theory and close relationships (pp. 77-114). New York: Guilford Press.

Fraley, R. C., Waller, N. G., \& Brennan, K. A. (2000). An item response theory analysis of selfreport measures of adult attachment. Journal of Personality and Social Psychology, 78, 350365.

Frankel, H., Snowden, L. R., \& Nelson, L. S. (1993). Wives adjustment to military deployment: An empirical evaluation of a family stress model. International Journal of Sociology of the Family, 22, 93-117. 
George, D., \& Mallery, P. (2003). SPSS for Windows step by step: A simple guide and reference. 11.0 update (4th ed.). Boston: Allyn \& Bacon.

Green, S., Nurius, P. S., \& Lester, P. (2013). Spouse psychological well-being: A keystone to military family health. Journal of Human Behavior in The Social Environment, 23(6), 1024.

Hazan, C., \& Shaver, P. R. (1987). Romantic love conceptualized as an attachment process. Journal of Personality and Social Psychology, 52, 511-524.

Hazan, C., \& Shaver, P.R. (1994). Attachment as an organizational framework for research on close relationships. Psychological Inquiry, 5, 1-22.

Hendrick, S. S. (1988). A generic measure of relationship satisfaction. Journal of Marriage and the Family, 50, 93-98.

Hinkle, D. E., Wiersma, W., \& Jurs, S. G. (2003). Applied statistics for the behavioural sciences (5th ed.). Boston, MA: Houghton Mifflin Company.

Isabella, R. A. (1993). Origins of attachment: Maternal interactive behavior across the first year. Child Development, 64(2), 605-621.

Kessler, R. C., Andrews, G., Colpe, L. J., et al. (2002). Short screening scales to monitor population prevalences and trends in non-specific psychological distress. Psychological Medicine, 32(6), 959-956.

Kirkpatrick, L. A., \& Davis, K. E. (1994). Attachment style, gender, and relationship stability: A longitudinal analysis. Journal of Personality and Social Psychology, 66, 502-512.

Kirkpatrick, L. A., \& Hazan, C. (1994). Attachment styles and close relationships: A four-year prospective study. Personal Relationships, 1, 123-142.

Kline, P. (2000). The handbook of psychological testing (2nd ed.). London: Routledge. 
Kobak, R., Cole, H. E., Ferenz-Gillies, R., Fleming, W. S., \& Gamble, S. Attachment and emotion regulation during mother-teen problem solving. A control theory analysis. Child Development, 64, 231-245.

Kobak, R. R., \& Hazan, C. (1991). Parents and spouses: Attachment strategies and marital functioning. University of Delaware, Newark.

Kurdek, L. A. (1991). Correlates of relationship satisfaction in cohabitating gay and lesbian couples: Integration of contextual, investment, and problem-solving models. Journal of Personality and Social Psychology, 61(6), 910-922.

Lamerson, C. D. (2001, September). Survey issues in TTCP nations (Report No. TTCP.HUM/01/10). Ottawa, Ontario, Canada: Department of National Defence, Directorate of Human Resource Research \& Evaluation.

Logan, K. V. (1987). The emotional cycle of deployment. Proceedings, U.S. Naval Institute.

Lussier, Y., Sabourin, S., \& Turgeon, C. (1999). Coping strategies as moderators of the relationship between attachment and marital adjustment. Journal of Social and Personal Relationships, 14, 777-791.

Lyons-Ruth, K., \& Jacobvitz, D. (2008). Attachment disorganization: Genetic factors, parenting contexts, and developmental transformation from infancy to adulthood. In J. Cassidy \& P. R. Shaver (Eds.), Handbook of attachment: Theory, research, and clinical applications (pp. 3- 20). New York: Guilford Press.

MacDonald, T. K., \& Ross, M. (1999). Assessing the accuracy of predictions about dating relationships: How and why do lovers' predictions differ from those made by observers? Personality and Social Psychology Bulletin, 25, 1417-1429.

Mayseless, O., Danieli, R., \& Sharbany, R. (1996). Adults' attachment patterns: Coping with 
separations. Journal of Youth and Adolescence, 25, 667-690.

McHugh, M. L. (2012). Interrater reliability: the kappa statistic. Biochemia Medica, 22(3), 276282.

Meltzer, A. L., McNulty, J. K., Jackson, G. L., \& Karney, B. L. (2014). Sex differences in the implications of partner physical attractiveness for the trajectory of marital satisfaction. Journal of Personality and Social Psychology, 106(3), 418-428.

Medway, F. J., Davis, K. E., Cafferty, T. P., Chappell, K. D., \& O’Hearn, R. E. (1995). Family disruption and adult attachment correlates of spouse and child reactions to separation and reunion due to Operation Desert Storm. Journal of Social and Child Psychology, 14, 97118.

Meyer, B., Olivier, L., \& Roth, D. A. (2005). Please don't leave me! BIS/BAS, attachment styles, and responses to a relationship threat. Personality and Individual Differences, 38, $151-162$.

Mikulincer, M. (1998). Adult attachment style and individual differences in functional versus dysfunctional experiences of anger. Journal of Personality and Social Psychology, 74, 513524.

Mikulincer, M., Birnbaum, G., Woddis, D., \& Nachmias, O. (2000). Stress and accessibility of proximity-related thoughts: Exploring the normative and intraindividual components of attachment theory. Journal of Personality and Social Psychology, 78(3), 509-523.

Mikulincer, M., Dolev, T., \& Shaver, P. R. (2004). Attachment-related strategies during thought suppression: Ironic rebounds and vulnerable self-representations. Journal of Personality and Social Psychology, 87(6), 940-956. 
Mikulincer, M., \& Florian, V. (1998). The relationship between adult attachment styles and emotional and cognitive reactions to stressful events. In J. A. Simpson \& W. S. Rholes (Eds.), Attachment theory and close relationships, (pp. 143-165). New York: Guilford Press.

Mikulincer, M., Florian, V., \& Wellar, A. (1993). Attachment styles, coping strategies, and posttraumatic psychological distress: The impact of the Gulf War in Israel. Journal of Personality and Social Psychology, 64, 817-826.

Mikulincer, M., Gillath, O., \& Shaver, P. R. (2002). Activation of the attachment system in adulthood: Threat-related primes increase the accessibility of mental representations of attachment figures. Journal of Personality and Social Psychology, 83, 881-895.

Mikulincer, M., Orbach, I., \& Iavnieli, D. (1998). Adult attachment style and affect regulation: Strategic variations in self-other similarity. Journal of Personality and Social Psychology, 75, 436-448.

Mikulincer, M., \& Shaver, P.R. (2003). The attachment behavioral system in adulthood: Activation, psychodynamics, and interpersonal processes. In M.P. Zanna (Ed.), Advances in experimental social psychology (pp. 53-152). New York: Academic Press.

Mikulincer, M., \& Shaver, P.R. (2007). Attachment in adulthood: Structure, dynamics, and change. New York: Guilford Press.

Mooney, C. Z., \& Duval, R. D. (1993). Bootstrapping : a nonparametric approach to statistical inference. Newbury Park, CA: Sage.

National Defence and the Canadian Armed Forces. (2015). Casualty Support. Retrieved from http://www.forces.gc.ca/en/caf-community-support-services-casualty-support/contactinfo.page. 
Neff, L. A. \& Geers, A. L. (2014). Optimistic expectations in early marriage: A resource or vulnerability for adaptive relationship functioning? Journal of Personality and Social Psychology, 105(1), 38-60.

Newell, C. E., Rosenfeld, P., Harris, R. W., \& Hindelang, R. L. (2004). Reasons for nonresponse on U.S. Navy surveys: a closer look. Military Psychology, 16(4), 265-276.

Norton, R. (1983). Measuring marital quality: A critical look at the dependent variable. Journal of Marriage and the Family, 45, 141-151.

Padden, D. L., Connors, R. A., \& Agazio, J. G. (2010). Stress, coping, and well-being in military spouses during deployment separation. Western Journal of Nursing Research, 33(2), 247267.

Painter, J. (year). Designing multilevel models using SPSS 11.5 mixed model. Retrieved from http://www.unc.edu/ painter/SPSSMixed/SPSSMixedModels.PDF

Pearlin, L. I., Liberman, M. A., Menaghan, E. G., \& Mullin, J. T. (1981). The stress process. Journal of Health and Social Behavior, 22(4), 337- 56.

Pearlin, L. I. \& Schooler, I. (1981). The structure of coping. Journal of Health and Social Behavior, 19(1), 2-21.

Preacher, K. J., \& Hayes, A. F. (2008). Asymptotic and resampling strategies for assessing and comparing indirect effects in multiple mediator models. Behavior Research Methods, 40, 879-891.

Preacher, K. J., Zhang, Z., \& Zyphur, M. J. (2011). Alternative methods for assessing mediation in multilevel data: The advantages of multilevel SEM. Structural Equation Modeling, 18, $161-182$. 
Rholes, W. S., Simpson, J. A., Campbell, L., \& Grich, J. (2001). Adult attachment and the transition to parenthood. Journal of Personality and Social Psychology, 81, 421-435.

Rosenberg, M. (1965). Society and the adolescent self-image. Princeton, NJ: Princeton University Press.

Scharfe, E., \& Bartholomew, K. (1994). Reliability and stability of adult attachment patterns. Personal Relationships, 1(1), 23-43.

Shaver, P., Schwartz, J., Kirson, D., \& O’Connor, C. (1987). Emotion knowledge: Further exploration of a prototype approach. Attitudes and Social Cognition, 52(6), 1061-1086.

Shrout, P. E., \& Bolger, N. (2002). Mediation in Experimental and Nonexperimental Studies: New Procedures and Recommendations. Psychological Methods, 7(4), 422-445.

Simpson, J. A. (1990). Influence of attachment styles on romantic relationships. Journal of Personality and Social Psychology, 59(5), 971-980.

Simpson, J. A., Rholes, W. S., \& Phillips, D. (1996). Conflict in close relationships: An attachment perspective. Journal of Personality and Social Psychology, 71(5), 899-914.

Spielberger, C. D., Gorsuch, R. L., Lushene, R., Vagg, P. R., \& Jacobs, G. A. (1983). Manual for the State-Trait Anxiety Inventory. Palo Alto, CA: Consulting Psychologists Press.

Sroufe, L. A., \& Waters, E. (1977). Heart rate as a convergent measure in clinical and developmental research. Merrill-Palmer Quartlery, 23, 3-27.

Tabachnick B. G., Fidell L. S. (2007). Using multivariate statistics (4th ed.). Needham Heights, MA: Allyn and Bacon.

Tidwell, M. O., Reis, H. T., \& Shaver, P. R. (1996). Attachment, attractiveness, and social interaction: A diary study. Journal of Personality and Social Psychology, 71(4), 729-745. 
The Royal Navy and Royal Marines Children's Fund. (2009). The overlooked casualties of conflict. A Report Commissioned by the Royal Navy and Royal Marine's Children's Fund. Van Ijzendoorn, M. H., Schuengel, C., \& Bakermans-Kranenburg, M. J. (1999). Disorganized attachment in early childhood: Meta-analysis of precursors, concomitants, and sequelae. Developmental and Psychopathology, 11(2), 225-250.

Waters, E., Merrick, S., Treboux, D., Crowell, J., \& Albersheim, L. (2000). Attachment security in infancy and early adulthood: A twenty-year longitudinal study. Child Development, 71, 684-689.

Watson, D., Clark, L. A., \& Tellegen, A. (1988). Development and validation of brief measures of positive and negative affect: The PANAS scales. Journal of Personality and Social Psychology, 54(6), 1063-1070.

Wei, M., Mallinckrodt, B., Larson, L. M., \& Zakalik, B. (2005). Adult attachment, depressive symptoms, and validation from self versus others. Journal of Counseling Psychology, 52(3), 368-277.

West, S. G., Finch, J. F., \& Curran, P. J. (1995). Structural equation models with nonnormal variables: Problems and remedies. In R. H. Hoyle (Ed.), Structural equation modeling: Concepts, issues and applications (pp. 56-75). Thousand Oaks, CA: Sage.

Wijngaards-de-Meij, L., Stroebe, M., Schut, H., Stroebe, W., van den Bout, J., van der Heijden, P. G. M., et al. (2007). Patterns of attachment and parents' adjustment to the death of their child. Personality and Social Psychology Bulletin, 33, 537-548. 


\section{Appendix A}

Instructions: Please respond to each statement by indicating how much you agree or disagree with it and checking the appropriate circle for each item.

$\begin{array}{ccccccc}\begin{array}{c}\text { Strongly } \\ \text { Disagree }\end{array} & \text { Disagree } & \begin{array}{c}\text { Slightly } \\ \text { Disagree }\end{array} & \begin{array}{c}\text { Neither } \\ \text { agree nor } \\ \text { disagree }\end{array} & \begin{array}{c}\text { Slightly } \\ \text { Agree }\end{array} & \text { Agree } & \begin{array}{c}\text { Strongly } \\ \text { Agree }\end{array} \\ \text { O } & \text { O } & \text { O } & \text { O } & \text { O } & \text { O } & \text { O }\end{array}$

1. I prefer not to show a partner how I feel deep down.

2. I often worry that my partner will not want to stay with me.

3. I am very comfortable being close to romantic partners.

4. I worry a lot about my relationships.

5. My partner really understands me and my needs.

6. I worry that romantic partners won't care about me as much as I care about them.

7. I get uncomfortable when a romantic partner wants to be very close.

8. I am afraid that I will lose my partner's love.

9. I don't feel comfortable opening up to romantic partners.

10. I often wish that my partner's feelings for me were as strong as my feelings for him/her.

11. It's not difficult for me to get close to my partner.

12. When I show my feelings for romantic partners, I'm afraid they will not feel the same about me.

13. I am nervous when partners get too close to me.

14. I rarely worry about my partner leaving me.

15. I feel comfortable sharing my private thoughts and feelings with my partner.

16. My desire to be very close sometimes scares people away.

17. It's easy for me to be affectionate with my partner.

18. I often worry that my partner doesn't really love me.

19. I find it relatively easy to get close to my partner.

20. I worry that I won't measure up to other people.

21. I find it difficult to allow myself to depend on romantic partners.

22. I do not often worry about being abandoned.

23. I prefer not to be too close to romantic partners.

24. It makes me mad that I don't get the affection and support that I need from my partner.

25. I tell my partner just about everything.

26. I find that my partner(s) don't want to get as close as I would like.

27. I usually discuss my problems and concerns with my partner.

28. My romantic partner makes me doubt myself.

29. I feel comfortable depending on romantic partners.

30. My partner only seems to notice me when I'm angry.

31. I find it easy to depend on romantic partners.

32. Sometimes romantic partners change their feelings about me for no apparent reason.

33. It helps to turn to my romantic partner in times of need.

34. I'm afraid that once a romantic partner gets to know me, he or she won't like who I really am.

35. I talk things over with my partner.

36. When my partner is out of sight, I worry that he or she might become interested in someone else. 


\section{Appendix B}

Instructions: Please respond to each statement by indicating how much you agree or disagree with it and checking the appropriate circle for each item.

$\begin{array}{ccccccc}\text { Strongly } & \text { Disagree } & \begin{array}{c}\text { Slightly } \\ \text { Disagree }\end{array} & \begin{array}{c}\text { Neither } \\ \text { agree nor } \\ \text { disagree }\end{array} & \begin{array}{c}\text { Slightly } \\ \text { Agree }\end{array} & \text { Agree } & \begin{array}{c}\text { Strongly } \\ \text { Agree }\end{array} \\ \text { O } & \text { O } & \text { O } & \text { O } & \text { O } & \text { O } & \text { O }\end{array}$

1. On the whole, I am satisfied with myself.

2. At times I think I am no good at all.

3. I feel that I have a number of good qualities.

4. I am able to do things as well as most other people.

5. I feel that I do not have much to be proud of.

6. I certainly feel useless at times.

7. I feel that I am a person of worth, at least equal with others.

8. I wish that I could have more respect for myself.

9. I usually feel that I am a failure.

10. I take a positive attitude toward myself. 


\section{Appendix C}

Instructions: These questions ask about your perceptions of your relationship with your current spouse/partner. Please respond to each statement by checking the appropriate circle for each item.

$\begin{array}{ccccccc}\begin{array}{c}\text { Strongly } \\ \text { Disagree }\end{array} & \text { Disagree } & \begin{array}{c}\text { Slightly } \\ \text { Disagree }\end{array} & \begin{array}{c}\text { Neither } \\ \text { agree nor } \\ \text { disagree }\end{array} & \begin{array}{c}\text { Slightly } \\ \text { Agree }\end{array} & \text { Agree } & \begin{array}{c}\text { Strongly } \\ \text { Agree }\end{array} \\ \text { O } & \text { O } & \text { O } & \text { O } & \text { O } & \text { O } & \text { O }\end{array}$

1. How well does your partner meet your needs?

2. In general, how satisfied are you with your relationship?

3. How good is your relationship compared to most?

4. How often do you wish you hadn't gotten into this relationship?

5. To what extent has your relationship met your original expectations?

6. How much do you love your partner?

7. How many problems are there in your relationship?

8 . How much do you trust your spouse? 


\section{Appendix D}

Instructions: Read each statement and select the appropriate response to indicate how you feel right now, that is, at this very moment. There is no right or wrong answer. Do not spend too much time on any one statement but select the answer which seems to describe your present feelings best.

Not at all

$\mathrm{O}$

A little

$\mathrm{O}$
Somewhat

$\mathrm{O}$
Very much so

$\mathrm{O}$

1. I feel calm

2. I feel secure

3. I feel tense

4. I feel strained

5. I feel at ease

6. I feel upset

7. I am presently worrying over possible misfortunes

8. I feel satisfied

9. I feel frightened

10. I feel uncomfortable

11. I feel self-confident

12. I feel nervous

13. I feel jittery

14. I feel indecisive

15. I am relaxed

16. I feel content

17. I am worried

18. I feel confused

19. I feel steady

20. I feel pleasant 


\section{Appendix E}

Instructions: The following scale consists of a number of words that describes different feelings and emotions. Indicate to what extent you currently feel this way by checking the appropriate circle for each item.

$\begin{array}{ccccc}\text { Not at all } & \text { A little } & \text { Moderately } & \text { Quite a bit } & \text { Extremely } \\ \mathrm{O} & \mathrm{O} & \mathrm{O} & \mathrm{O} & \mathrm{O}\end{array}$

1. interested

2. distressed

3. excited

4. upset

5. strong

6. guilt

7. scared

8. hostile

9. enthusiastic

10. proud

11. irritable

12. alert

13. ashamed

14. inspired

15. nervous

16. determined

17. attentive

18. jittery

19. active

20. afraid 


\section{Appendix F}

Instructions: The following questions concern your perceptions of your current spouse/partner and relationship. Please indicate the extent to which you agree or disagree with the following statements and check the appropriate circle for each item.

$\begin{array}{ccccccc}\begin{array}{c}\text { Strongly } \\ \text { Disagree }\end{array} & \text { Disagree } & \begin{array}{c}\text { Slightly } \\ \text { Disagree }\end{array} & \begin{array}{c}\text { Neither } \\ \text { agree nor } \\ \text { disagree }\end{array} & \begin{array}{c}\text { Slightly } \\ \text { Agree }\end{array} & \text { Agree } & \begin{array}{c}\text { Strongly } \\ \text { Agree }\end{array} \\ \text { O } & \text { O } & \text { O } & \text { O } & \text { O } & \text { O } & \text { O }\end{array}$

1. Sometimes I feel unsure about continuing this relationship.

2. I never tire of my spouse's company, no matter how much time we spend together.

3. I find that I become even closer to my partner when external events threaten our relationship.

4. I find that as I discover my partner's faults, I sometimes question our relationship.

5. I expect that I will become even happier and more satisfied with my relationship over time.

6. Over time, it is likely that I will discover areas in which my needs conflict with those of my partner.

7. I do not like some aspects of my partner's personality.

8. My partner is physically attractive.

9. I sometimes try to change things about my partner (e.g., attitudes, behaviours).

10. I feel valued by my partner.

11. My partner provides me with emotional support.

12. I am satisfied with my partner's attitudes and values.

13. My partner is kind and understanding.

14. My partner is easy to get along with.

15. I am satisfied with my partner's social status.

16. My partner is a "good match" for me.

17. If this relationship were to end, I could find an equally satisfactory romantic partner.

18. I could get by without a romantic relationship.

19. Whether this relationship continues depends primarily on factors within my control.

20. My partner and I communicate well.

21. My partner and I have similar goals. 


\section{Appendix G}

Instructions: Please answer these questions considering how you feel right now by indicating the extent to which you agree or disagree with the following statements and checking the appropriate circle for each item.

$\begin{array}{ccccccc}\begin{array}{c}\text { Strongly } \\ \text { Disagree }\end{array} & \text { Disagree } & \begin{array}{c}\text { Slightly } \\ \text { Disagree }\end{array} & \begin{array}{c}\text { Neither } \\ \text { agree nor } \\ \text { disagree }\end{array} & \begin{array}{c}\text { Slightly } \\ \text { Agree }\end{array} & \text { Agree } & \begin{array}{c}\text { Strongly } \\ \text { Agree }\end{array} \\ \text { O } & \text { O } & \text { O } & \text { O } & \text { O } & \text { O } & \text { O }\end{array}$

1. I am satisfied with my life.

2. I am satisfied with my marriage.

3. I am feeling healthy.

4. I am feeling upbeat.

5. I have been feeling "down".

6. I feel good about myself.

7. I feel good about my financial situation.

8. I feel good about my social life.

9. I feel supported.

10. I can manage the ups and downs of everyday life.

11. I am full of energy.

12. I feel overwhelmed.

13. I have been sleeping well.

14. I feel lonely.

Items added for monthly survey:

14. I feel content most of the time.

15. I have been sleeping well.

16. I cope with physical pain daily.

17. I feel nauseous most of the time.

18. My body often aches.

19. I make an effort to eat well.

20. I make an effort to eat well.

20. I exercise regularly (i.e., $3 \mathrm{x} /$ week).

I wish I had more help around the house. 


\section{Appendix $\mathrm{H}$}

Instructions: The following statements concern how you have been feeling about the relationship since your current spouse/partner has been home. Please indicate the appropriate response below.

12. Has your partner's return met your expectations?

\begin{tabular}{|c|c|c|c|c|c|c|}
\hline $\begin{array}{c}1 \\
\text { Not at all }\end{array}$ & 2 & 3 & $\begin{array}{c}4 \\
\text { Somewhat }\end{array}$ & 5 & 6 & 7 \\
\hline $\mathrm{O}$ & $\mathrm{O}$ & $\mathrm{O}$ & $\mathrm{O}$ & $\mathrm{O}$ & $\mathrm{O}$ & $\mathrm{O}$ \\
\hline
\end{tabular}

13. Relative to the time before deployment, do you now feel more or less satisfied with your relationship?

\begin{tabular}{|c|c|c|c|c|c|c|}
\hline $\begin{array}{c}1 \\
\text { A lot less } \\
\text { satisfied }\end{array}$ & 2 & 3 & $\begin{array}{c}4 \\
\text { The same } \\
\text { as before }\end{array}$ & 5 & 6 & $\begin{array}{c}7 \\
\text { A lot more } \\
\text { satisfied }\end{array}$ \\
\hline $\mathrm{O}$ & $\mathrm{O}$ & $\mathrm{O}$ & $\mathrm{O}$ & $\mathrm{O}$ & $\mathrm{O}$ & $\mathrm{O}$ \\
\hline
\end{tabular}

14. Relative to the time before deployment, do you now feel more or less emotionally connected to your partner?

\begin{tabular}{|c|c|c|c|c|c|c|}
\hline $\begin{array}{c}\text { I feel a lot } \\
\text { more } \\
\text { emotionally } \\
\text { distant } \\
\text { from my } \\
\text { partner }\end{array}$ & 2 & 3 & $\begin{array}{c}\text { I feel the } \\
\text { same as } \\
\text { before }\end{array}$ & 5 & 6 & $\begin{array}{c}\text { I feel a lot } \\
\text { closer to my } \\
\text { partner }\end{array}$ \\
\hline $\mathrm{O}$ & $\mathrm{O}$ & $\mathrm{O}$ & $\mathrm{O}$ & $\mathrm{O}$ & $\mathrm{O}$ & $\mathrm{O}$ \\
\hline
\end{tabular}

15. Relative to the time while your partner was deployed, are things running more or less smoothly around the house?

\begin{tabular}{|c|c|c|c|c|c|c|}
\hline $\begin{array}{l}1 \\
\text { Things are } \\
\text { running } \\
\text { much less } \\
\text { smoothly }\end{array}$ & 2 & 3 & $\begin{array}{c}4 \\
\text { Things are } \\
\text { running the } \\
\text { same as } \\
\text { before }\end{array}$ & 5 & 6 & $\begin{array}{c}7 \\
\text { Things are } \\
\text { running much } \\
\text { more smoothly }\end{array}$ \\
\hline $\mathrm{O}$ & $\mathrm{O}$ & $\mathrm{O}$ & $\mathrm{O}$ & $\mathrm{O}$ & $\mathrm{O}$ & $\mathrm{O}$ \\
\hline
\end{tabular}


16. Overall, how have your interactions been with your partner?

\begin{tabular}{|c|c|c|c|c|c|c|}
\hline $\begin{array}{c}1 \\
\text { Extremely } \\
\text { Unpleasant }\end{array}$ & 2 & 3 & $\begin{array}{c}4 \\
\text { Neutral }\end{array}$ & 5 & 6 & $\begin{array}{c}7 \\
\text { Extremely } \\
\text { pleasant }\end{array}$ \\
\hline $\mathrm{O}$ & $\mathrm{O}$ & $\mathrm{O}$ & $\mathrm{O}$ & $\mathrm{O}$ & $\mathrm{O}$ & $\mathrm{O}$ \\
\hline
\end{tabular}

17. Since your partner's return, how often have you experienced conflict?

\begin{tabular}{|c|c|c|c|c|c|c|}
\hline $\begin{array}{c}1 \\
\text { Not at all }\end{array}$ & 2 & 3 & $\begin{array}{c}4 \\
\text { Sometimes }\end{array}$ & 5 & 6 & $\begin{array}{c}7 \\
\text { All the time }\end{array}$ \\
\hline $\mathrm{O}$ & $\mathrm{O}$ & $\mathrm{O}$ & $\mathrm{O}$ & $\mathrm{O}$ & $\mathrm{O}$ & $\mathrm{O}$ \\
\hline
\end{tabular}

18. If you have experienced conflict with your spouse/partner, what are the main issues of conflict? 


\section{Appendix I}

Instructions: Please answer these questions considering how you feel right now by indicating the extent to which you agree or disagree with the following statements and checking the appropriate circle for each item.

$\begin{array}{ccccccc}\begin{array}{c}\text { Strongly } \\ \text { Disagree }\end{array} & \text { Disagree } & \begin{array}{c}\text { Slightly } \\ \text { Disagree }\end{array} & \begin{array}{c}\text { Neither } \\ \text { agree nor } \\ \text { disagree }\end{array} & \begin{array}{c}\text { Slightly } \\ \text { Agree }\end{array} & \text { Agree } & \begin{array}{c}\text { Strongly } \\ \text { Agree }\end{array} \\ \text { O } & \text { O } & \text { O } & \text { O } & \text { O } & \text { O } & \text { O }\end{array}$

1. I am feeling healthy.

2. I am feeling upbeat.

3. I have been feeling "down".

4. I feel good about myself.

5. I feel good about my financial situation.

6. I feel good about my social life.

7. I feel supported.

8. I can manage the ups and downs of everyday life.

9. I am full of energy.

10. I feel overwhelmed.

11. I have been sleeping well.

12. I feel lonely.

13. I feel content most of the time.

14. I have been sleeping well.

15 . I cope with physical pain daily.

16. I feel nauseous most of the time.

17. My body often aches.

18. I make an effort to eat well.

19. I exercise regularly (i.e., 3x/week).

20. I wish I had more help around the house. 


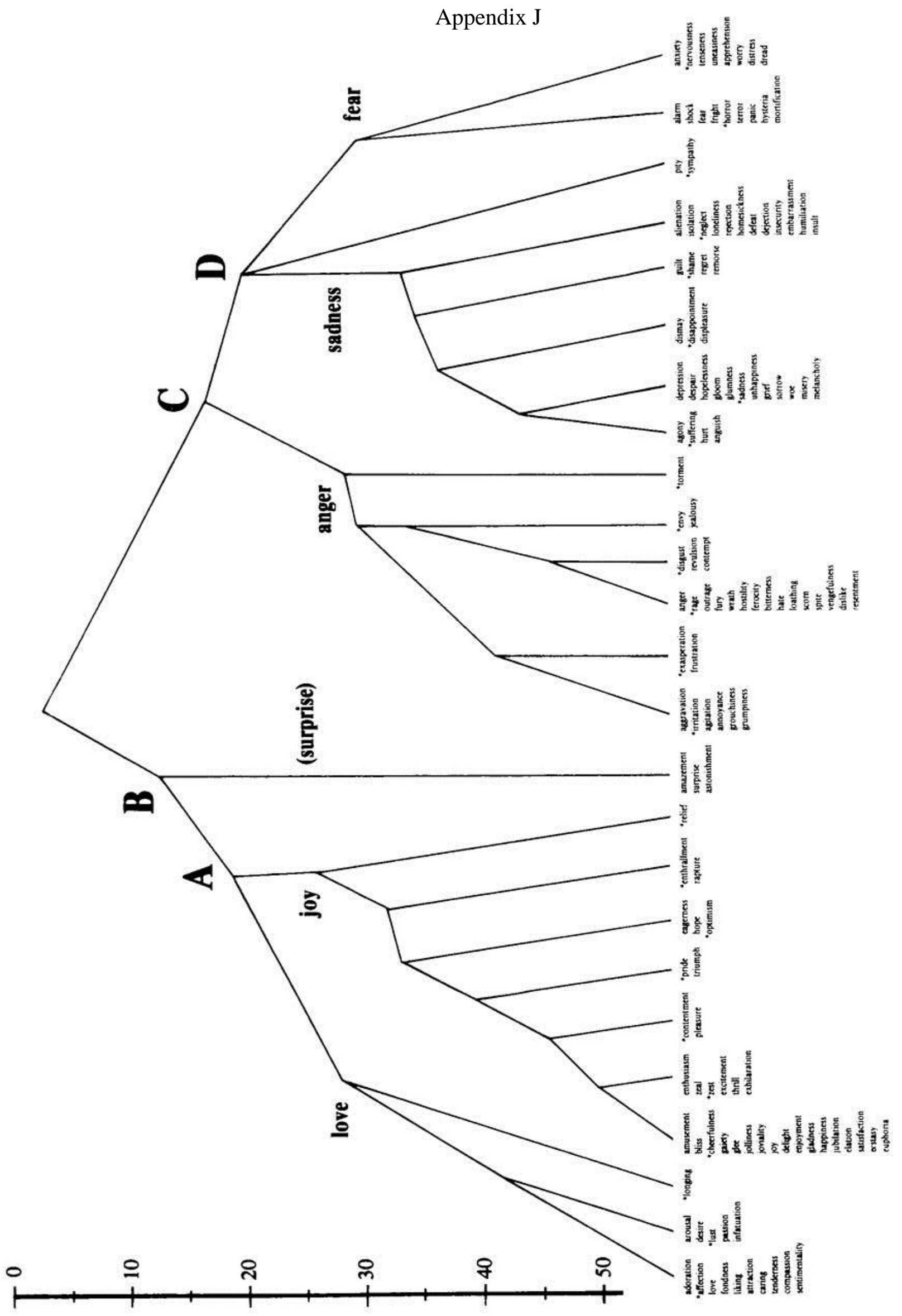




\section{Appendix K}

Instructions: To which degree the following describes the impact of military life on your own career during your relationship with your military spouse/partner?

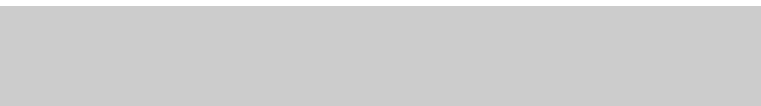

\section{Definite Probabl Uncerta Probabl Definite} lynot ynot in yyes ly yes

1. My spouse/partner's military career has

$\mathrm{O}$

$\mathrm{O}$

$\mathrm{O}$

$\mathrm{O}$

$\mathrm{O}$

$\mathrm{O}$ had a positive impact on my employment or career

2. My employment or career has not been affected by my spouse/partner's military career

3. I have made some employment or career sacrifices because of my spouse/partner's military career

4. I am under-qualified for the work I am doing because of my spouse/partner's military career

5. I am over-qualified for the work I am doing because of my spouse/partner's military career

6. I am unemployed or my career has been

\begin{tabular}{|l|l|l|l|l|l|}
\hline & 0 & 0 & 0 & 0 & 0 \\
\hline & & & & \\
\hline
\end{tabular}

$\mathrm{O}$

O

$\mathrm{O}$

$\mathrm{O}$

$\mathrm{O}$

$\mathrm{O}$ severely affected by the demands of my spouse/partner's military career

\begin{tabular}{|l|l|l|l|l|l|}
\hline & $\mathrm{O}$ & $\mathrm{O}$ & $\mathrm{O}$ & $\mathrm{O}$ & $\mathrm{O}$ \\
\hline & & & \\
\hline
\end{tabular}

$\begin{array}{llllll}\mathrm{O} & \mathrm{O} & \mathrm{O} & \mathrm{O} & \mathrm{O} & \mathrm{O}\end{array}$

$\begin{array}{llllll}\mathrm{O} & \mathrm{O} & \mathrm{O} & \mathrm{O} & \mathrm{O} & \mathrm{O}\end{array}$ 


\section{Appendix L}

Instructions: For each of the following possible stressful situations you and your family experienced in the past 12 months, please indicate how stressful you felt it was for you and your family.

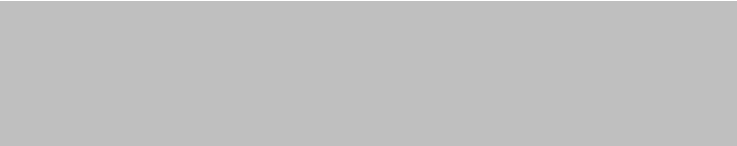

1. A combat-related deployment or duty assignment for your spouse/partner

2. A non-combat-related deployment or duty assignment requiring your spouse/partner to be away from home

3. Uncertainty about future deployments or duty assignments

4. Combat-related injury to your spouse/partner

5. A non-combat-related injury to your spouse/partner from carrying out his/her military duties

6. Caring for your ill, injured, or disabled spouse/partner

7. Intensified training schedule for your spouse/partner

8. Increased time your spouse/partner spent away from family, or missed family celebrations, while performing military duties

9. Family conflict over whether spouse/partner should remain in the military or reserves

10. Difficult balancing demands of family life and your spouse/partner's military duties
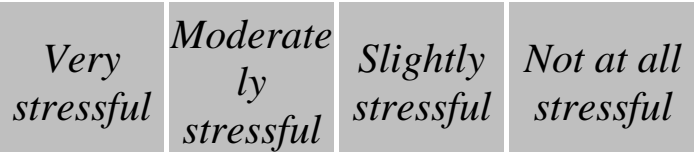

Never experienc

$\mathrm{O}$

$\mathrm{O}$

$\mathrm{O}$

$\mathrm{O}$

$\mathrm{O}$

$\mathrm{O}$

O

O

O

$\mathrm{O}$

O

$\mathrm{O}$

O

O

O

O

O

O

O

O

O

O

O

O

O

O

O

O

O

O

O

O

O

O

O

O

O

O

O

O

O

$\mathrm{O}$

O

O

O

O

$\mathrm{O}$

O

O

$\mathrm{O}$ 


\section{Appendix M}

Instructions: The following questions ask about stress you may experience on a day-to-day basis. Please select the one that best describes your answer.

1. Thinking about the amount of stress in your life, would you say that most days are:

\begin{tabular}{|c|c|c|c|c|}
\hline $\begin{array}{c}1 \\
\text { Not at all } \\
\text { stressful }\end{array}$ & $\begin{array}{c}2 \\
\text { Not very } \\
\text { stressful }\end{array}$ & $\begin{array}{c}3 \\
\text { A bit } \\
\text { stressful }\end{array}$ & $\begin{array}{c}4 \\
\text { Quite a bit } \\
\text { stressful }\end{array}$ & $\begin{array}{c}5 \\
\text { Extremely } \\
\text { stressful }\end{array}$ \\
\hline $\mathrm{O}$ & $\mathrm{O}$ & $\mathrm{O}$ & $\mathrm{O}$ & $\mathrm{O}$ \\
\hline
\end{tabular}

2. In general, how would you rate your ability to handle unexpected and difficult problems (e.g., a family or personal crisis)?

\begin{tabular}{|c|c|c|c|c|}
\hline 1 & 2 & 3 & 4 & 5 \\
Poor & Fair & Good & Very good & $\begin{array}{c}5 \\
\text { Excellent }\end{array}$ \\
\hline O & O & O & O & O \\
\hline
\end{tabular}

3. In general, how would you rate your ability to handle the day-to-day demands in your life (e.g., handling work, family and volunteer responsibilities)?

\begin{tabular}{|c|c|c|c|c|}
\hline $\begin{array}{c}1 \\
\text { Poor }\end{array}$ & $\begin{array}{c}2 \\
\text { Fair }\end{array}$ & $\begin{array}{c}3 \\
\text { Good }\end{array}$ & $\begin{array}{c}4 \\
\text { Very good }\end{array}$ & $\begin{array}{c}5 \\
\text { Excellent }\end{array}$ \\
\hline $\mathrm{O}$ & $\mathrm{O}$ & $\mathrm{O}$ & $\mathrm{O}$ & $\mathrm{O}$ \\
\hline
\end{tabular}

4. Do you have the desire to eat when you are stressed or emotionally upset?

\begin{tabular}{|c|c|c|c|c|}
\hline $\begin{array}{c}1 \\
\text { Never }\end{array}$ & $\begin{array}{c}2 \\
\text { Seldom }\end{array}$ & $\begin{array}{c}3 \\
\text { Sometimes }\end{array}$ & $\begin{array}{c}4 \\
\text { Often }\end{array}$ & $\begin{array}{c}5 \\
\text { Very } \\
\text { often }\end{array}$ \\
\hline $\mathrm{O}$ & $\mathrm{O}$ & $\mathrm{O}$ & $\mathrm{O}$ & $\mathrm{O}$ \\
\hline
\end{tabular}




\section{Appendix N}

Instructions: Please respond to the following statements by indicating how much you agree or disagree with them.

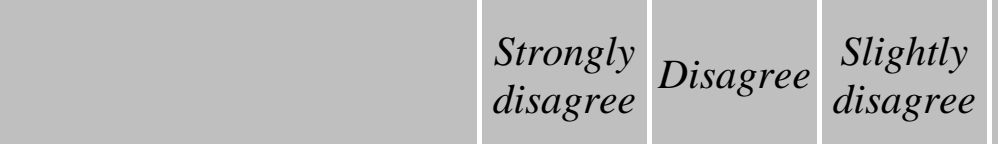

\begin{tabular}{c|c|c|c}
$\begin{array}{c}\text { Neither } \\
\text { agree nor } \\
\text { disagree }\end{array}$ & $\begin{array}{c}\text { Slightly } \\
\text { agree }\end{array}$ & Agree & $\begin{array}{c}\text { Strongly } \\
\text { agree }\end{array}$ \\
\end{tabular}

1. In most ways my life is

$\mathrm{O}$

$\mathrm{O}$

$\mathrm{O}$

$\mathrm{O}$

$\mathrm{O}$

$\mathrm{O}$ close to ideal.

$\mathrm{O} \quad \mathrm{O} \quad \mathrm{O}$

O

O

O

O life are excellent.

3. I am satisfied with my life.

$\mathrm{O} \quad \mathrm{O} \quad \mathrm{O}$

$\mathrm{O}$

$\mathrm{O}$

$\mathrm{O}$

O

4. So far I have gotten the important things I want

O

$\mathrm{O}$

$\mathrm{O}$

O

O

O

O in life.

5. If I could live my life O

O

O

O

O

O

O over, I would change almost nothing. 


\section{Appendix O}

Instructions: The following ten questions ask about how often you have been feeling in the past four weeks. For each question, please mark the circle under the option that best describes the amount of time that you felt that way.

\begin{tabular}{|c|c|c|c|c|c|}
\hline $\begin{array}{l}\text { In the past four weeks, about how often } \\
\text { did you feel }\end{array}$ & $\begin{array}{l}\text { None of } \\
\text { the time }\end{array}$ & $\begin{array}{l}\text { A little of } \\
\text { the time }\end{array}$ & $\begin{array}{l}\text { Some of } \\
\text { the time }\end{array}$ & $\begin{array}{l}\text { Most of } \\
\text { the time }\end{array}$ & $\begin{array}{c}\text { All of the } \\
\text { time }\end{array}$ \\
\hline
\end{tabular}

1. Tired-out for no good reason?

\begin{tabular}{|c|c|c|c|}
\hline $\mathrm{O}$ & $\mathrm{O}$ & $\mathrm{O}$ & $\mathrm{O}$ \\
\hline $\mathrm{O}$ & $\mathrm{O}$ & $\mathrm{O}$ & $\mathrm{O}$ \\
\hline $\mathrm{O}$ & $\mathrm{O}$ & $\mathrm{O}$ & $\mathrm{O}$ \\
\hline
\end{tabular}

3. So nervous that nothing could calm you

$\mathrm{O}$

$\mathrm{O}$

O

$\mathrm{O}$ down?

4. Hopeless?

$\mathrm{O}$

\begin{tabular}{l|l|l|l}
\hline & $\mathrm{O}$ & $\mathrm{O}$ & $\mathrm{O}$
\end{tabular}

5. Restless or fidgety?

$\mathrm{O} \quad \mathrm{O}$

$\mathrm{O}$

$\mathrm{O}$

6. So restless that you could not sit still?

$\mathrm{O}$

$\mathrm{O}$

$\mathrm{O}$

$\mathrm{O}$

$\mathrm{O}$

7. Depressed?

$\mathrm{O} \quad \mathrm{O}$

$\mathrm{O}$

$\mathrm{O}$

$\mathrm{O}$

8. That everything was an effort?

$\mathrm{O}$

$\mathrm{O}$

$\mathrm{O}$

$\mathrm{O}$

$\mathrm{O}$

9. So sad that nothing could cheer you up?

$\mathrm{O}$

$\mathrm{O}$

$\mathrm{O}$

$\mathrm{O}$

$\mathrm{O}$

10. Worthless?

$\mathrm{O}$

$\mathrm{O}$

$\mathrm{O}$

$\mathrm{O}$

$\mathrm{O}$ 


\section{Appendix P}

Instructions: Please rate your level of agreement with the following statements about yourself.

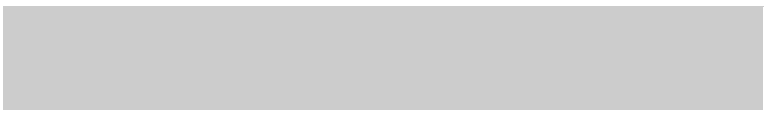

1. There is really no way I can solve some of the problems I have.

2. Sometimes I feel that I'm being pushed around in life.

3. I have little control over the things that happen to me.

4. I can do just about anything I really set my mind to.

5. I often feel helpless in dealing with the problems of life.

6. What happens to me in the future mostly depends on me.

7. There is little I can do to change many of the important things in my life.

\section{Strongly disagree

\begin{tabular}{l|l} 
Disagree & Agree \\
$\begin{array}{c}\text { Strongly } \\
\text { agree }\end{array}$
\end{tabular}

$\mathrm{O}$

$\mathrm{O}$

$\mathrm{O}$

$\mathrm{O}$

\begin{tabular}{|l|l|l|l|}
\hline & $\mathrm{O}$ & $\mathrm{O}$ & $\mathrm{O}$ \\
\hline
\end{tabular}

$\begin{array}{llll}\mathrm{O} & \mathrm{O} & \mathrm{O}\end{array}$

\begin{tabular}{|l|l|l|l|}
\hline & $\mathrm{O}$ & $\mathrm{O}$ & $\mathrm{O}$
\end{tabular}

$\begin{array}{llll}\mathrm{O} & \mathrm{O} & \mathrm{O}\end{array}$

\begin{tabular}{|l|l|l|l|}
\hline O & $\mathrm{O}$ & $\mathrm{O}$ & $\mathrm{O}$ \\
\hline
\end{tabular}

$\mathrm{O}$

\begin{abstract}
$\mathrm{O}$
\end{abstract}
$\mathrm{O}$

$\mathrm{O}$ 


\section{Appendix Q}

Instructions: We are interested in how people respond when they confront difficult or stressful events in their lives. Different events can bring out somewhat different responses, but think about what you have been doing while you are under a lot of stress. Please indicate to what extent you have been engaging in the following ways to deal with stress.
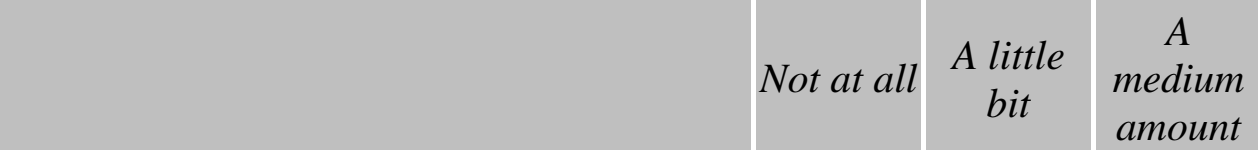

A lot

1. I've been turning to work or other

$\mathrm{O}$

$\mathrm{O}$

$\mathrm{O}$

$\mathrm{O}$ activities to take my mind off things.

2. I've been concentrating my efforts on doing something about the situation I'm

$\mathrm{O}$

$\mathrm{O}$

$\mathrm{O}$

$\mathrm{O}$ in.

3. I've been saying to myself "this isn't

$\mathrm{O}$

$\mathrm{O}$

$\mathrm{O}$

$\mathrm{O}$ real.".

4. I've been using alcohol or other drugs to

$\mathrm{O}$

$\mathrm{O}$

$\mathrm{O}$

$\mathrm{O}$ make myself feel better.

5. I've been getting emotional support from

$\mathrm{O}$

$\mathrm{O}$

$\mathrm{O}$

$\mathrm{O}$ others.

6. I've been giving up trying to deal with it.

$\mathrm{O}$

$\mathrm{O}$

$\mathrm{O}$

$\mathrm{O}$

7. I've been taking action to try to make the

$\mathrm{O}$

$\mathrm{O}$

$\mathrm{O}$

$\mathrm{O}$ situation better.

8. I've been refusing to believe that it has happened.

9. I've been saying things to let my

$\mathrm{O}$

$\mathrm{O}$

$\mathrm{O}$

$\mathrm{O}$ unpleasant feelings escape.

10. I've been getting help and advice from other people.

O O O O

\begin{tabular}{l|l|l|l}
\hline & $\mathrm{O}$ & $\mathrm{O}$ & $\mathrm{O}$
\end{tabular}

11. I've been using alcohol or other drugs to help me get through it.

12. I've been trying to see it in a different light, to make it seem more positive.

$\mathrm{O} \quad \mathrm{O} \quad \mathrm{O}$

\begin{tabular}{l|l|l|l|}
\hline $\mathrm{O}$ & $\mathrm{O}$ & $\mathrm{O}$ & $\mathrm{O}$
\end{tabular}

13. I've been criticizing myself.

O O O O

14. I've been trying to come up with a strategy about what to do.

\begin{tabular}{l|l|l|l}
\hline & $\mathrm{O}$ & $\mathrm{O}$ & $\mathrm{O}$
\end{tabular}

15. I've been getting comfort and

$\mathrm{O}$ understanding from someone.

16. I've been giving up the attempt to cope.

$\mathrm{O}$

$\mathrm{O}$

$\mathrm{O}$

$\mathrm{O}$ 
17. I've been looking for something good in
what is happening.

\begin{tabular}{|l|l|l|l|l|}
\hline 18. I've been making jokes about it. & $\mathrm{O}$ & $\mathrm{O}$ & $\mathrm{O}$ & $\mathrm{O}$ \\
\hline
\end{tabular}

19. I've been doing something to think about $\quad \mathrm{O} \quad \mathrm{O} \quad \mathrm{O} \quad \mathrm{O}$ it less, such as going to movies,

20. I've been watching TV, reading, daydreaming, sleeping, or shopping.

21. I've been accepting the reality of the fact $\quad \mathrm{O} \quad \mathrm{O} \quad \mathrm{O} \quad \mathrm{O}$ that it has happened.

22. I've been expressing my negative

\begin{tabular}{|l|l|l|l}
\hline $\mathrm{O}$ & $\mathrm{O}$ & $\mathrm{O}$ & $\mathrm{O}$
\end{tabular}
feelings.

23. I've been trying to find comfort in my religion or spiritual beliefs.

$\begin{array}{llll}\mathrm{O} & \mathrm{O} & \mathrm{O}\end{array}$

24. I've been trying to get advice or help

\begin{tabular}{l|l|l|l}
\hline & $\mathrm{O}$ & $\mathrm{O}$ & $\mathrm{O}$
\end{tabular}
from other people about what to do.

25. I've been learning to live with it.

$\mathrm{O}$

$\mathrm{O}$

$\mathrm{O} \quad \mathrm{O}$

26. I've been thinking hard about what steps

$\mathrm{O}$

$\mathrm{O}$

$\mathrm{O}$

O to take.

27. I've been blaming myself for things that happened.

28. I've been praying or meditating.

O

O

O

O

$\mathrm{O}$

$\mathrm{O}$

$\mathrm{O}$

$\mathrm{O}$ 
Appendix R

Instructions: Please rate your level of agreement with the following each statement as it describes your social strengths, skills, and practices.
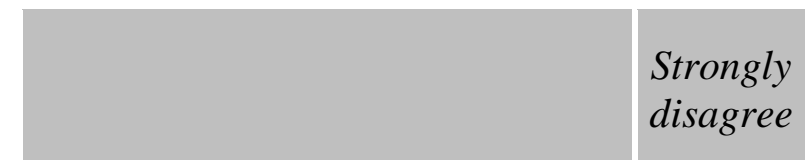

$\mathrm{O}$ spouse/partner
1. I am loved and cared for by my

2. I receive support and encouragement from my spouse/partner

3. I can depend on my spouse/partner for help or assistance, if I request it

4. I maintain a healthy diet

5. I exercise on a regular basis

6. I maintain a healthy lifestyle

7. I look forward to beginning each day

8. I maintain a positive outlook on life

9. I enjoy most days

10. When I face adversities, I try to understand the situation and my options before I act

11. When I face adversities, I apply lessons learned from my past experiences to inform my actions

12. When I face adversities, I think carefully about the consequences of my response before I act

13. I am willing to turn to other people in the community for help or assistance, if I need it

14. I am willing to turn to leaders in my spouse/partner's unit for help or assistance, if I need it.

15. I am willing to turn to support

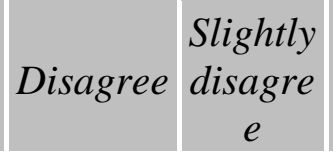

\section{Slightly agree}

Agree

Strongly agree

$\mathrm{O}$

$\mathrm{O}$

$\mathrm{O}$

$\mathrm{O}$

$\begin{array}{llllll}\mathrm{O} & \mathrm{O} & \mathrm{O} & \mathrm{O} & \mathrm{O} & \mathrm{O}\end{array}$

$\mathrm{O}$

$\mathrm{O}$

$\mathrm{O}$

$\mathrm{O}$

$\mathrm{O}$

O

O O

O

\begin{abstract}
O
\end{abstract}
O

O

O $\mathrm{O}$

$\mathrm{O}$

$\mathrm{O}$

$\mathrm{O}$

$\mathrm{O}$

O O

$\mathrm{O}$

$\mathrm{O}$

$\mathrm{O}$

$\mathrm{O}$

O $\mathrm{O}$

$\mathrm{O}$

O

O

O

O

O

O

O

O

O

\begin{tabular}{|l|l|l|l|l|l|}
\hline $\mathrm{O}$ & $\mathrm{O}$ & $\mathrm{O}$ & $\mathrm{O}$ & $\mathrm{O}$ & $\mathrm{O}$ \\
\hline $\mathrm{O}$ & $\mathrm{O}$ & $\mathrm{O}$ & $\mathrm{O}$ & $\mathrm{O}$ & $\mathrm{O}$
\end{tabular}

$\mathrm{O}$

$\mathrm{O}$

$\mathrm{O}$

$\mathrm{O}$

$\mathrm{O}$

$\mathrm{O}$

$\mathrm{O}$

$\mathrm{O}$

$\mathrm{O}$

O

O

O

O O

O

O

O

O

O

O

O

O

O

O
O

O

O

O

O

O 
agencies and organizations for

help or assistance, if I need it.

16. I have a guiding set of principles or beliefs

17. I attempt to live in accordance with a guiding set of principles or beliefs

18. I draw strength from a guiding set of principles or beliefs when I face life challenges

19. I am able to handle day-to-day family matters if my spouse/partner is unavailable

20. I am able to cope emotionally if my spouse/partner is unavailable

21. I know where to turn for assistance or support if my spouse/partner is unavailable

22. I understand the demands of my spouse/partner's military job

23. I am supportive of my spouse/partner serving in or working for the military

24. I am proud to be the

$\mathrm{O} \quad \mathrm{O}$

O

$\mathrm{O}$

$\mathrm{O}$

$\mathrm{O}$
$\mathrm{O}$
$\mathrm{O}$
$\mathrm{O}$
$\mathrm{O}$
$\mathrm{O}$
$\mathrm{O}$

$\mathrm{O}$

$\mathrm{O}$

$\mathrm{O}$

$\mathrm{O}$

$\mathrm{O}$

$\mathrm{O}$

(1)

\begin{tabular}{|l|l|l|l|l|l|}
\hline O & O & O & O & 0 & 0 \\
\hline
\end{tabular}

$\begin{array}{llllll}\mathrm{O} & \mathrm{O} & \mathrm{O} & \mathrm{O} & \mathrm{O} & \mathrm{O}\end{array}$

\begin{tabular}{|l|l|l|l|l|l|}
\hline O & O & O & O & O & O \\
\hline
\end{tabular}

$\begin{array}{llllll}\mathrm{O} & \mathrm{O} & \mathrm{O} & \mathrm{O} & \mathrm{O} & \mathrm{O}\end{array}$
$\mathrm{O}$
$\mathrm{O}$
$\mathrm{O}$
O
$\mathrm{O}$
$\mathrm{O}$ spouse/partner of a person serving in or working for the military

25. I keep a positive perspective when I am going through a difficult time

26. I confront the problem directly when I face a challenge or difficulty
27 . I cope successfully with stress in my life

28. I look forward to spending time together with my spouse/partner

29. I am happy with my relationship with my spouse/partner

30. I am committed to making my relationship with my spouse/partner a success

$\begin{array}{llllll}\mathrm{O} & \mathrm{O} & \mathrm{O} & \mathrm{O} & \mathrm{O} & \mathrm{O}\end{array}$

\begin{tabular}{|l|l|l|l|l|l|}
\hline O & $\mathrm{O}$ & $\mathrm{O}$ & $\mathrm{O}$ & $\mathrm{O}$ & $\mathrm{O}$ \\
\hline
\end{tabular}

$\begin{array}{llllll}\mathrm{O} & \mathrm{O} & \mathrm{O} & \mathrm{O} & \mathrm{O} & \mathrm{O}\end{array}$

\begin{tabular}{|l|l|l|l|l|l|}
\hline O & O & O & O & O & O \\
\hline O & O & O & O & O & O \\
\hline
\end{tabular}

\begin{tabular}{|l|l|l|l|l|l|}
\hline O & $\mathrm{O}$ & $\mathrm{O}$ & $\mathrm{O}$ & $\mathrm{O}$ & $\mathrm{O}$ \\
\hline $\mathrm{O}$ & $\mathrm{O}$ & $\mathrm{O}$ & $\mathrm{O}$ & $\mathrm{O}$ & $\mathrm{O}$ \\
\hline
\end{tabular}




\section{Appendix S}

Instructions: Please rate your level of agreement with the following statements about your relationship with your spouse/partner.
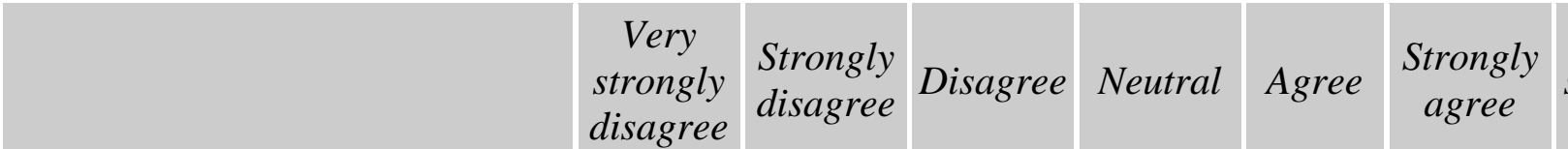

Very strongly

1. I have a good

$\mathrm{O}$

$\mathrm{O}$

$\mathrm{O}$

$\mathrm{O}$

$\mathrm{O}$

$\mathrm{O}$ agree marriage/relationship

2. My marriage/relationship with

O

$\mathrm{O}$

O

$\mathrm{O}$

$\mathrm{O}$

$\mathrm{O}$

$\mathrm{O}$ my spouse/partner is very stable

3. My marriage/relationship with

$\mathrm{O}$

$\mathrm{O}$

$\mathrm{O}$

O

$\mathrm{O}$

$\mathrm{O}$

$\mathrm{O}$ my spouse/partner makes me happy

4. Our marriage/relationship is

$\mathrm{O}$

$\mathrm{O}$

$\mathrm{O}$

$\mathrm{O}$

$\mathrm{O}$

$\mathrm{O}$

$\mathrm{O}$ strong

5. I really feel like a part of a

$\mathrm{O}$

$\mathrm{O}$

$\mathrm{O}$

$\mathrm{O}$

$\mathrm{O}$

$\mathrm{O}$

$\mathrm{O}$ team with my spouse/partner

6. Everything considered, there

$\mathrm{O}$

$\mathrm{O}$

$\mathrm{O}$

$\mathrm{O}$

$\mathrm{O}$

O

$\mathrm{O}$ could not be more happiness in our marriage/relationship. 


\title{
Appendix T
}

Instructions: Most people have disagreements in their relationships. Please rate your level of agreement with the following items about your relationship with your spouse/partner.
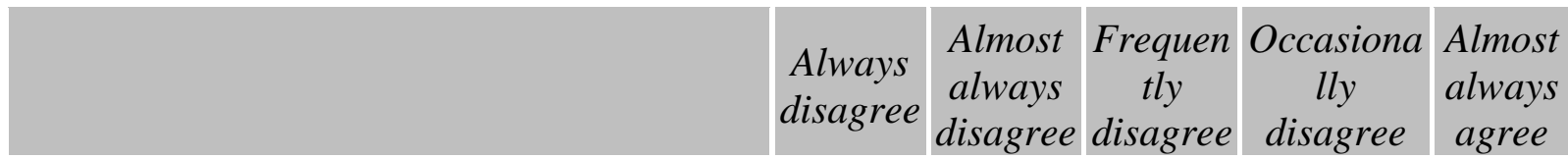

Always agree

1. Religious matters

$$
\mathrm{O}
$$

$$
\mathrm{O}
$$

$\mathrm{O}$

$\mathrm{O}$

$\mathrm{O} \quad \mathrm{O}$

2. Demonstrations of affection

$\mathrm{O}$

$\mathrm{O}$

$\mathrm{O}$

$\mathrm{O}$

O

$\mathrm{O}$

3. Making major decisions

O O

O

O

$\mathrm{O} \quad \mathrm{O}$

4. Sex Relations

$\mathrm{O}$

$\mathrm{O}$

$\mathrm{O}$

$\mathrm{O}$

$\mathrm{O}$

5. Conventionality (correct or proper

O O

O

$\mathrm{O}$

$\mathrm{O}$

$\mathrm{O}$

6. Career decisions

O

\begin{abstract}
$\mathrm{O}$
\end{abstract}
$\mathrm{O}$

$\mathrm{O}$

O

O

Never

7. How often do you discuss or have you considered divorce, separation, or terminating your relationship?

8. How often do you and your spouse/partner quarrel?

9. Do you ever regret that you married (or lived together)?

10. How often do you and your spouse/partner "get on each other's nerves"?

$\mathrm{O}$ Rarely Occasiona More often $\mathrm{O}$ lly than not

\section{Most of the time}

All the time

\begin{tabular}{|l|l|l|l|l|l|}
\hline O & O & O & O & O & O \\
\hline 0 & 0 & 0 & 0 & 0 & 0 \\
\hline & 0 & 0 & 0 & 0 & 0 \\
\hline
\end{tabular}
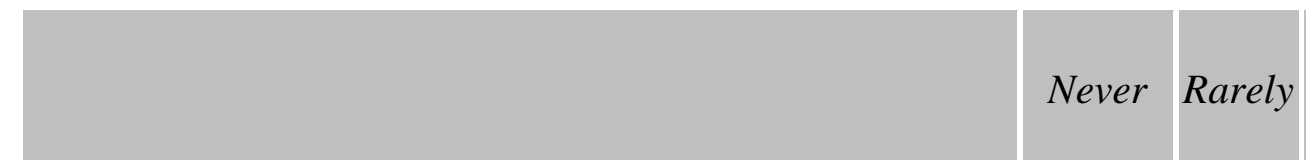
Occasiona
lly Almost
every
day

11. Do you and your spouse/partner engage in outside $\mathrm{O}$ $\mathrm{O}$ O $\mathrm{O}$ $\mathrm{O}$ interests together? 


\section{Appendix U}

General Research Ethics Board Letter of Approval for Phases One and Two

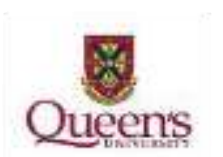

Finimg 25, 2012

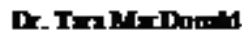

Depirting of Pighthing

Enmpeyin

Quand Uimaty

Fing ON ET IN6

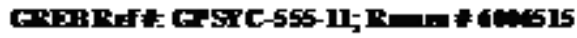

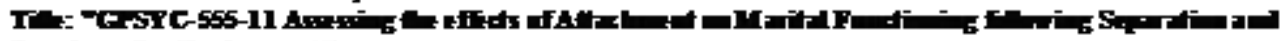

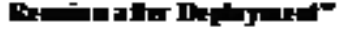

Desr Dr.M-Domi:

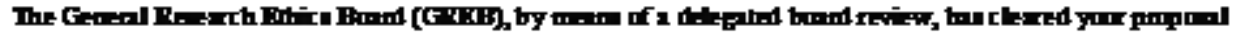

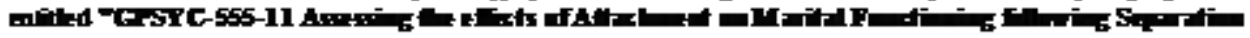

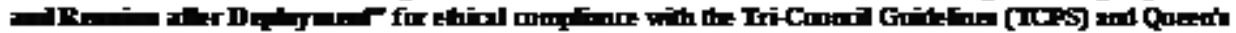

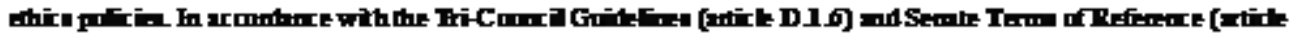

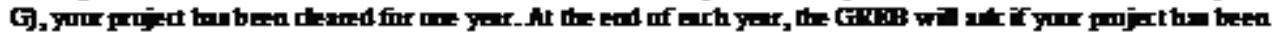

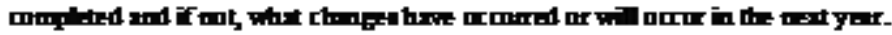

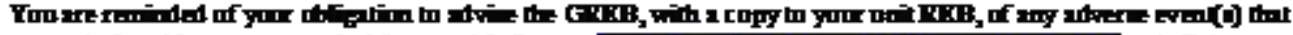

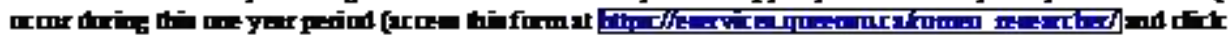

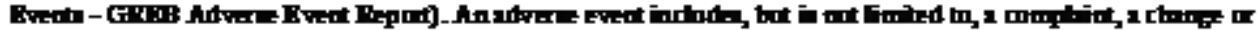

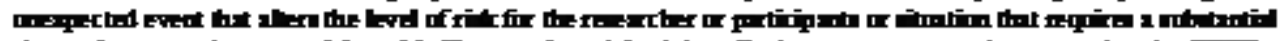

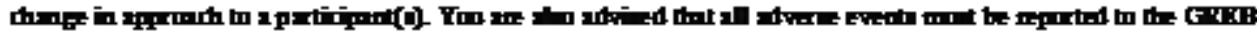

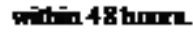

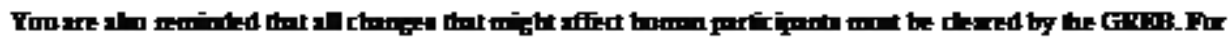

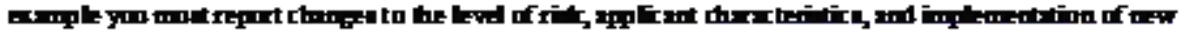

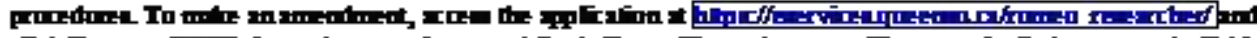

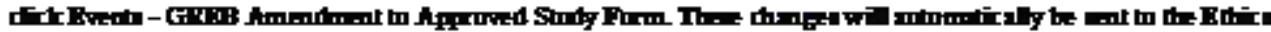

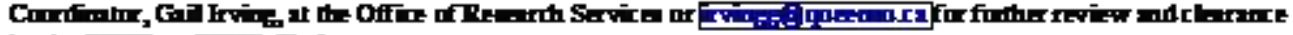

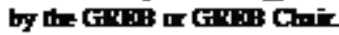

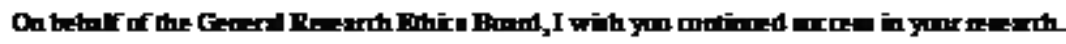

Toun timenty

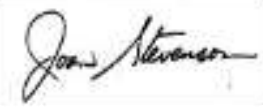

Inin Sirve on, FhD.

Pinfer an Chir

Genon P.

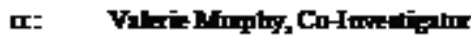

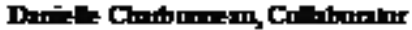

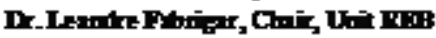

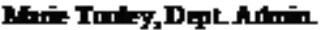




\title{
Appendix V
}

\section{General Research Ethics Board Letter of Approval for Phase Three}

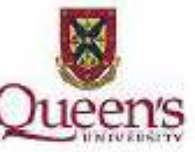

July 21,2015

\author{
Ms. Valerie Murphy \\ $\mathrm{Ph}$ D. Candidate \\ Department of Psychology \\ Queen's University \\ Humphrey Hall \\ Kingston, ON, K 7L 3N6
}

GREB Ref \#: GPSYC-718-15; Romeo \# 6016050

Title: "GPSYC-718-15 Adult Attachment and Reports of Intrapersonal Well-Being and Relationship Quality in Spouses of Military Members"

Dear Ms. Murphy:

The General Research Ethics Board (GREB), by means of a delegated board review, has cleared your proposal entitled "GPSYC-718-15 Adult Attachment and Reports of Intrapersonal Well-Being and Relationship Quality in Spouses of Military Members" for ethical compliance with the Tri-Council Guidelines (TCPS) and Queen's ethics policies. In accordance with the Tri-Council Guidelines (article D.1.6) and Senate Terms of Reference (article $G$ ), your project has been cleared for one year. At the end of each year, the GREB will ask if your project has been completed and if not, what changes have occurred or will occur in the next year.

You are reminded of your obligation to advise the GREB, with a copy to your unit REB, of any adverse event(s) that occur during this one year period (access this form at https://eservices.queensu.ca/romeo researcher/ and click Events - GREB Adverse Event Report). An adverse event includes, but is not limited to, a complaint, a change or unexpected event that alters the level of risk for the researcher or participants or situation that requires a substantial change in approach to a participant(s). You are also advised that all adverse events must be reported to the GREB within 48 hours.

You are also reminded that all changes that might affect human participants must be cleared by the GREB. For example you must report changes to the level of risk, applicant characteristics, and implementation of new procedures. To make an amendment, access the application at https://eservices.queensu.ca/romeo_researcher/ and click Events - GREB Amendment to Approved Study Form. These changes will automatically be sent to the Ethics Coordinator, Gail Irving, at the Office of Research Services or irvings a queensuca for further review and clearance by the GREB or GREB Chair.

On behalf of the General Research Ethics Board, I wish you continued success in your research.

Yours sincerely,

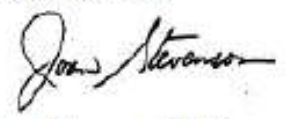

Joan Stevenson, $\mathrm{Ph} . \mathrm{D}$.

Chair

General Research Ethics Board

c: Dr. Tara MacDonald, Faculty Supervisor

Dr. Stanka Fitneva, Chair, Unit REB

Ms. Marie Tooley, Dept. Admin. 